\title{
$k$-Schur functions and affine Schubert calculus
}

first released September 2012, last updated October 2013

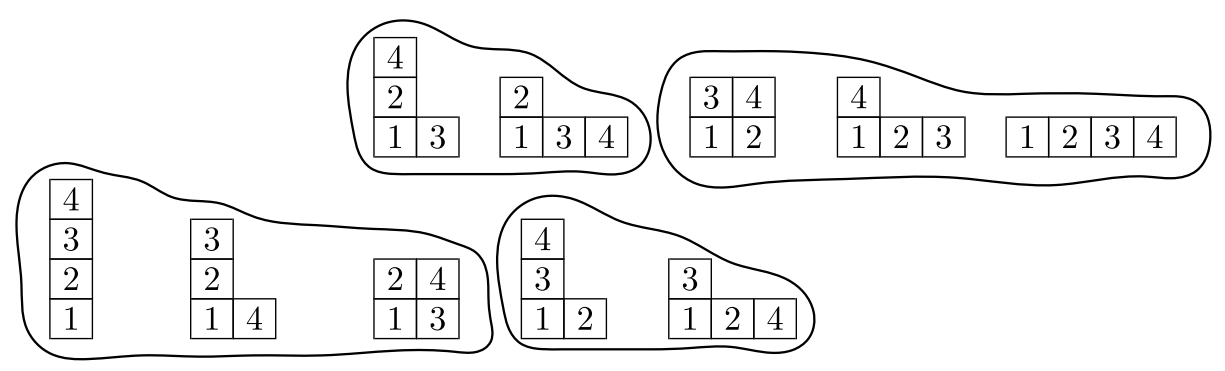

Thomas Lam

Luc Lapointe Jennifer Morse Anne Schilling Mark Shimozono Mike Zabrocki

Copyright (c) 2012 by the authors.

These lecture notes may be reproduced in their entirety for non-commercial purposes. 


\section{Contents}

1 Introduction $\quad 6$

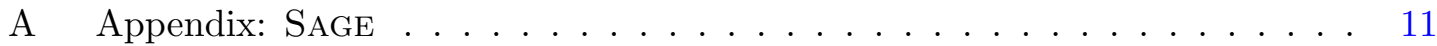

2 Primer on $k$-Schur Functions $\quad 13$

1 Background and notation . . . . . . . . . . . . . . . 14

1.1 Partitions and cores . . . . . . . . . . . . . . . . 14

1.2 Bounded partitions, cores, and affine Grassmannian elements . . . . 18

1.3 Weak order and horizontal chains . . . . . . . . . . . 26

1.4 Cores and the strong order of the affine symmetric group . . . . . . 30

1.5 Symmetric functions . . . . . . . . . . . . . . . . . 35

$1.6 \quad$ Schur functions . . . . . . . . . . . . . . . . 38

1.7 Hall-Littlewood symmetric functions . . . . . . . . . . . . . . . . . 41

1.8 Macdonald symmetric functions . . . . . . . . . . . . . . 43

1.9 Empirical approach to $k$-Schur functions . . . . . . . . . . . . . 47

1.10 Notes on references . . . . . . . . . . . . . . . . . . . 51

2 From Pieri rules to $k$-Schur functions at $t=1 \ldots \ldots \ldots \ldots$

2.1 Semi-standard tableaux and a monomial expansion of Schur functions 52

2.2 Weak tableaux and a monomial expansion of dual $k$-Schur functions $\quad 55$

2.3 Other realizations . . . . . . . . . . . . . . . 60

2.4 Strong marked tableaux and a monomial expansion of $k$-Schur functions 64

$2.5 \quad k$-Littlewood-Richardson coefficients . . . . . . . . . . . . 69

$2.6 \quad$ Notes on references . . . . . . . . . . . . . . . . 73

3 Definitions of $k$-Schur functions . . . . . . . . . . . . . . . . . . 74

3.1 Atoms as tableaux . . . . . . . . . . . . . . . . 74

3.2 A symmetric function operator definition . . . . . . . . . . 80

3.3 Weak tableaux II . . . . . . . . . . . . . . . . . . 82

3.4 Strong tableaux II . . . . . . . . . . . . . . . . . 86

$3.5 \quad$ Notes on references . . . . . . . . . . . . . . . . . . . 89

4 Properties of $k$-Schur functions and their duals . . . . . . . . . . . . 90

$4.1 \quad k$-Schur functions are Schur functions when $k \geq|\lambda|$ and when $t=0 . \quad 90$ 
4.2 The $k$-Schur function is Schur positive . . . . . . . . . . . . 91

4.3 At $t=1$, the $k$-Schur functions satisfy the $k$-Pieri rule . . . . . . 92

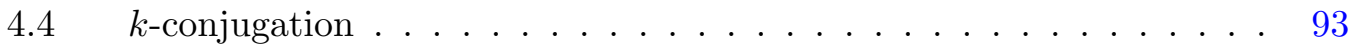

4.5 The $k$-Schur functions form a basis for $\Lambda_{(k)}^{t} \ldots \ldots \ldots \ldots 9$

4.6 The $k$-rectangle property . . . . . . . . . . . . . . 96

4.7 When $t=1$, the product of $k$-Schur functions is $k$-Schur positive . . 96

4.8 Positively closed under coproduct . . . . . . . . . . . . 97

4.9 The product of a $k$-Schur and $\ell$-Schur function is $(k+\ell)$-Schur positive 98

4.10 Branching property from $k$ to $k+1 \ldots \ldots$. . . . . . . . . 99

$4.11 k$-Schur positivity of Macdonald symmetric functions . . . . . . . . . 100

$5 \quad$ Directions of research and open problems . . . . . . . . . . . . . . 102

5.1 A $k$-Murnaghan-Nakayama rule . . . . . . . . . . . . . . . . . . . 102

$5.2 \quad$ A rectangle generalization at $t$ a root of unity . . . . . . . . . 104

5.3 A dual-basis to $s_{\lambda}^{(k)}[X ; t] \ldots \ldots \ldots \ldots \ldots$

$5.4 \quad$ A product on $\Lambda_{(k)}^{t} \ldots \ldots \ldots \ldots \ldots \ldots$

5.5 A representation theoretic model of $k$-Schur functions . . . . . . 110

$5.6 \quad$ From Pieri to $K$-theoretic $k$-Schur functions . . . . . . . . . . . . . 111

6 Duality between the weak and strong orders . . . . . . . . . . . . 115

$6.1 \quad k$-analogue of the Cauchy identity . . . . . . . . . . . 115

6.2 A brief introduction to Fomin's growth diagrams . . . . . . . . . 118

6.3 Affine insertion . . . . . . . . . . . . . . . . . 121

6.4 The $t$-compatible affine insertion algorithm . . . . . . . . . . . 128

7 The $k$-shape poset . . . . . . . . . . . . . . . . . . . 129

3 Stanley symmetric functions and Peterson algebras $\quad 135$

1 Stanley symmetric functions and reduced words . . . . . . . . . . . . 137

1.1 Young tableaux and Schur functions . . . . . . . . . . . . . 137

1.2 Permutations and reduced words . . . . . . . . . . . . . 138

1.3 Reduced words for the longest permutation . . . . . . . . . . . . 138

1.4 The Stanley symmetric function . . . . . . . . . . . . . . . 138

1.5 The code of a permutation . . . . . . . . . . . . . 139

1.6 Fundamental Quasi-symmetric functions . . . . . . . . . . . . . . 140

$1.7 \quad$ Exercises . . . . . . . . . . . . . . . . . . . . 140

2 Edelman-Greene insertion . . . . . . . . . . . . . . . . . . . 141

2.1 Insertion for reduced words . . . . . . . . . . . . . . . . . 141

2.2 Coxeter-Knuth relations . . . . . . . . . . . . . . . . 143

2.3 Exercises and Problems . . . . . . . . . . . . . . . . . 143

3 Affine Stanley symmetric functions . . . . . . . . . . . . . . . . . . 145

3.1 Affine symmetric group . . . . . . . . . . . . . . . . 145

3.2 Definition . . . . . . . . . . . . . . . . . 146

$3.3 \quad$ Codes . . . . . . . . . . . . . . . . . . . 147 
$3.4 \Lambda_{(n)}$ and $\Lambda^{(n)} \ldots \ldots \ldots \ldots \ldots \ldots \ldots \ldots$

3.5 Affine Schur functions . . . . . . . . . . . . . . . . . . . 148

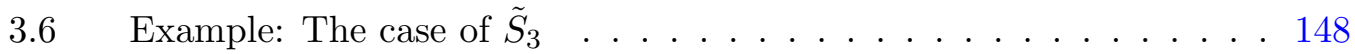

$3.7 \quad$ Exercises and problems . . . . . . . . . . . . . . . . 149

4 Root systems and Weyl groups . . . . . . . . . . . . . . . 151

4.1 Notation for root systems and Weyl groups . . . . . . . . . . . . . 151

$4.2 \quad$ Affine Weyl group and translations . . . . . . . . . . . . . . . 152

$5 \quad$ NilCoxeter algebra and Fomin-Stanley construction . . . . . . . . . . . 153

5.1 The nilCoxeter algebra . . . . . . . . . . . . . . 153

$5.2 \quad$ Fomin and Stanley's construction . . . . . . . . . . . . . . . 153

5.3 A conjecture . . . . . . . . . . . . . . . 155

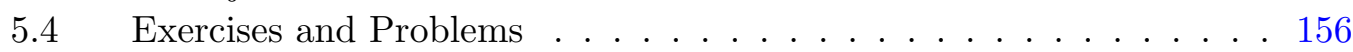

6 The affine nilHecke ring . . . . . . . . . . . . . 157

6.1 Definition of affine nilHecke ring . . . . . . . . . . . 157

$6.2 \quad$ Coproduct . . . . . . . . . . . . . . . . . 158

$6.3 \quad$ Exercises and Problems . . . . . . . . . . . . . . . 159

$7 \quad$ Peterson's centralizer algebras . . . . . . . . . . . . . . . . . 159

7.1 Peterson algebra and $j$-basis $\ldots \ldots \ldots \ldots$. . . . . . . . . . . . . . . . . . . . . 160

7.2 Sketch proof of Theorem 7.3 . . . . . . . . . . . . . 160

$7.3 \quad$ Exercises and Problems . . . . . . . . . . . . . . 161

8 (Affine) Fomin-Stanley algebras . . . . . . . . . . . . . . . . . 162

8.1 Commutation definition of affine Fomin-Stanley algebra . . . . . . . 162

$8.2 \quad$ Noncommutative $k$-Schur functions . . . . . . . . . . . . . . 163

8.3 Cyclically decreasing elements . . . . . . . . . . . . . . . . . . 164

8.4 Coproduct . . . . . . . . . . . . . . . . 165

$8.5 \quad$ Exercises and Problems . . . . . . . . . . . . . 165

9 Finite Fomin-Stanley subalgebra . . . . . . . . . . . . . . 166

$9.1 \quad$ Problems . . . . . . . . . . . . . . . . . . . 168

10 Geometric interpretations . . . . . . . . . . . . 168

4 Affine Schubert calculus $\quad 170$

1 Introduction . . . . . . . . . . . . . . . . . 170

2 Root Data . . . . . . . . . . . . . . . . . . 172

2.1 Cartan Data and the Weyl group . . . . . . . . . . . . . . . . 172

2.2 Root data . . . . . . . . . . . . . . . . . . 173

$2.3 \quad$ Affine root data . . . . . . . . . . . . . . 176

3 NilHecke ring and Schubert calculus . . . . . . . . . . . . . 180

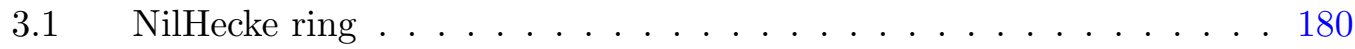

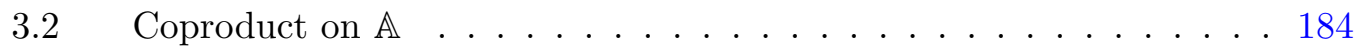

3.3 Duality and the GKM ring . . . . . . . . . . . . . . . 187

3.4 Multiplication in $\Lambda$ and coproduct in $\mathbb{A} \ldots \ldots . \ldots 191$ 
3.5 Forgetting equivariance . . . . . . . . . . . . . . . . . 192

3.6 Parabolic case . . . . . . . . . . . . . . . . . . 193

3.7 Geometric interpretations . . . . . . . . . . . . . . . 194

4 Affine Grassmannian . . . . . . . . . . . . . . . . . . . . 196

4.1 Affine Grassmannian as partial affine flags . . . . . . . . . . . . . . . 197

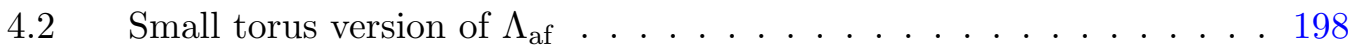

4.3 Homology of the affine Grassmannian . . . . . . . . . . . . . . . . 199

4.4 Small torus affine nilHecke ring and Peterson subalgebra . . . . . . . 200

4.5 The $j$-basis . . . . . . . . . . . . . . . . . 203

4.6 Homology structure constants . . . . . . . . . . . . . . . . . 204

4.7 Peterson's "Quantum Equals Affine" Theorems . . . . . . . . . . . . 205

A Appendix: Proof of coalgebra properties . . . . . . . . . . . . . . . 205

B Appendix: Small torus GKM proofs . . . . . . . . . . . . . . . 207

B.1 Small torus GKM condition for $\hat{\mathrm{sl}}_{2} \ldots \ldots$. . . . . . . . . . . . 209

C Appendix: Homology of $\mathrm{Gr} \ldots \ldots \ldots \ldots$. . . . . . . . . . 211 


\section{Chapter 1}

\section{Introduction}

Affine Schubert calculus is a subject that lies at the crossroads of combinatorics, geometry, and representation theory. Its modern development is motivated by two seemingly unrelated directions. One is the introduction of $k$-Schur functions in the study of Macdonald polynomial positivity, a mostly combinatorial branch of symmetric function theory. The other direction is the study of the Schubert bases of the (co)homology of the affine Grassmannian, an algebro-topological formulation of a problem in enumerative geometry.

Classical Schubert calculus is a branch of enumerative algebraic geometry concerned with problems of the form:

How many lines $L$ in 3 -space intersect four fixed lines $L_{1}, L_{2}, L_{3}, L_{4}$ ?

In general, lines are replaced by affine linear subspaces, and conditions on the dimensions of intersections are imposed. When $L_{1}, L_{2}, L_{3}, L_{4}$ are in generic position, the answer to the above problem is two; this is a pleasant surprise, since in linear algebra one expects to find 0,1 , or $\infty$ solutions. Schubert [145] studied such "Schubert problems" in the 19th century. At the turn of the 20th century, Hilbert posed as his fifteenth problem the rigorous foundation of Schubert's enumerative calculus. Subsequent developments in geometry and topology converted such Schubert problems into problems of computation in the cohomology ring $H^{*}(\operatorname{Gr}(k, n))$ of the Grassmannian $\operatorname{Gr}(k, n)$ of $k$-planes in $n$-space. The problems were reduced to finding structure constants, now called Littlewood-Richardson coefficients [118], of a certain "Schubert basis" for $H^{*}(\operatorname{Gr}(k, n))$.

The explicit realization of these computations using the theory of Schur functions played an important role in transforming Schubert calculus into a contemporary theory that stretches into many fields. The Schur functions $s_{\lambda}$ form a basis for the symmetric function space $\Lambda$ and at the turn of the century, it was discovered that they match irreducible representations of the symmetric group. Later, a deep connection between Schur functions and the geometry of Grassmannians was established when it was shown that the Schubert structure constants exactly equal coefficients in the product of Schur functions in $\Lambda$. The rich combinatorial backbone of the theory of Schur functions, including the 
Robinson-Schensted algorithm, jeu-de-taquin, the plactic monoid (see for example [140]), crystal bases [128], and puzzles [74], now underlies Schubert calculus and in particular produces a direct formula for the Littlewood-Richardson coefficients. The influence of Schur functions on the geometry of Grassmannians provoked the broadening of Schubert calculus to other studies ranging from representation theory to physics.

A trend in Schubert calculus is to generalize the classical setup in two basic directions: (1) to vary the underlying geometric object being considered by replacing the Grassmannian by the flag variety, or more generally by a partial flag variety of a Kac-Moody group, and (2) to vary the algebraic structure considered by replacing cohomology by equivariant cohomology, $K$-theory, quantum cohomology, or other algebraic invariants. Our interest is in the case when the Grassmannian is replaced by infinite-dimensional spaces $\operatorname{Gr}_{G}$ known as affine Grassmannians.

Investigations of the quantum cohomology rings of flag varieties led Peterson [131] to begin a systematic study in this direction for any complex simple simply-connected algebraic group G. Applying work of Kostant and Kumar [75] on the topology of Kac-Moody flag varieties, Peterson showed that the equivariant homology $H_{T}\left(\mathrm{Gr}_{G}\right)$ is isomorphic to a subalgebra of Kostant and Kumar's nilHecke ring. Moreover, he proved that the LittlewoodRichardson coefficients of $H_{T}\left(\mathrm{Gr}_{G}\right)$ could be identified with the 3-point Gromov-Witten invariants of the flag variety of $G$. A classical result of Quillen [136] establishes that the affine Grassmannian $\mathrm{Gr}_{G}$ is itself homotopy-equivalent to the group $\Omega K$ of based loops into the maximal compact subgroup $K \subset G$. This places $\operatorname{Gr}_{G}$ in a unique position amongst the homogeneous spaces of all Kac-Moody groups. It endows $H_{T}\left(\mathrm{Gr}_{G}\right)$ with the structure of a Hopf algebra, and is also partly responsible for the important position that the affine Grassmannian has in geometric representation theory.

The aim of this book is to present ongoing work developing a theory of affine Schubert calculus in the spirit of classical Schubert calculus; here the Grassmannian is replaced by the affine Grassmannian. As with Schubert calculus, topics under the umbrella of affine Schubert calculus are vast, but now it is the combinatorics of a family of polynomials called $k$-Schur functions that underpins the theory.

The theory of $k$-Schur functions originated in the apparently unrelated study of Macdonald polynomials. Macdonald polynomials are symmetric functions over $\mathbb{Q}(q, t)$ that possess remarkable properties; the proofs of which have inspired deep work in many areas (e.g. double affine Hecke algebras [31], quantum relativistic systems [139], Hilbert schemes of points in the plane [61]). Macdonald conjectured in the late 80's that the coefficients expressing Macdonald polynomials in terms of the Schur basis lie in $\mathbb{N}[q, t]$. Since then, the Macdonald/Schur transition coefficients have been intensely studied from a combinatorial, representation theoretic, and algebro-geometric perspective.

In one such study [92], Lapointe, Lascoux, and Morse found computational evidence for a family of new bases for subspaces $\Lambda_{k}^{t}$ in a filtration $\Lambda_{1}^{t} \subset \Lambda_{2}^{t} \subset \cdots \subset \Lambda_{\infty}^{t}$ of $\Lambda$. Conjecturally, the star feature of each basis was the property that Macdonald polynomials expand positively in terms of it, giving a remarkable factorization for the Macdonald/Schur 
transition matrices over $\mathbb{N}[q, t]$. Pursuant investigations of these bases led to various conjecturally equivalent characterizations and the discovery that they refined the very aspects of Schur functions that make them so fundamental and wide-reaching. As such, they are now generically called $k$-Schur functions.

The role of $k$-Schur functions in affine Schubert calculus emerged over a number of years. The springboard was a realization that the combinatorial backbone of $k$-Schur theory lies in the setting of the type- $A$ affine Weyl group. Generalizing the classical theory of Schur functions, Pieri rules, Young's lattice, the Cauchy identity, tableaux, and Stanley symmetric functions were refined using $k$-Schur functions [95, 96, 78]. These are naturally described in terms of posets of elements in $\tilde{A}_{k}$. For example, the number of monomial terms in an entry of the Macdonald/ $k$-Schur matrix equals the number of reduced expressions for an element in $\tilde{A}_{k}$.

The combinatorial exploration fused into a geometric one when the $k$-Schur functions were connected to the quantum cohomology of Grassmannians. Lapointe and Morse [97] showed that each Gromov-Witten invariant for the quantum cohomology of Grassmannians exactly equals a $k$-Schur coefficient in the product of $k$-Schur functions in $\Lambda$. A basis of dual (or affine) $k$-Schur functions was also introduced in [97]. In response to questions about the geometric role for dual $k$-Schur functions and the significance of the complete set of $k$-Schur coefficients, Morse and Shimozono conjectured that the Schubert bases for cohomology and homology of the affine Grassmannian Gr are given by the dual $k$-Schur functions and the $k$-Schur functions, respectively. Lam proved the conjectures in [79]. Since then, the synthesis of affine Schubert calculus and $k$-Schur function theory has produced a subject involving prolific research in mathematics, computer science, and physics.

This book arose from an NSF funded Focused Research Group entitled "Affine Schubert Calculus: Combinatorial, geometric, physical, and computational aspects", which involved Thomas Lam, Luc Lapointe, Jennifer Morse, Anne Schilling, Mark Shimozono, Nicolas M. Thiéry, and Mike Zabrocki as active participants among others. Our exposition here grew out of several lecture series given at a summer school on 'Affine Schubert Calculus' organized by Anne Schilling and Mike Zabrocki and held in July 2010 at the Fields Institute in Toronto.

We give the story in three parts, through varying lenses. Chapter 2 presents the origins and early work on $k$-Schur functions, emphasizing the symmetric function setting and the combinatorics therein. The computational aspects are highlighted and illustrated with examples in SAgE $[152,141]$. More information about the open-source computer algebra system SAGE is given in Appendix A. Chapter 3 is Thomas Lam's synopsis of his summer school lectures entitled "affine Stanley symmetric functions". This chapter explains the combinatorial connections between Stanley symmetric functions and $k$-Schur functions via the algebraic constructions of nilCoxeter and nilHecke rings. Some of the latter constructions are presented for arbitrary root systems. Chapter 4 is Mark Shimozono's synopsis of his lectures on "generalizations to other affine group types". This chapter presents the 
nilHecke ring in the very general Kac-Moody setting and develops some of the geometric connections. The general construction is then applied to the situation of the affine Grassmannian. Each chapter is self-contained and can in principle be read independently of the others.

Let us outline the contents of this book. As discussed, the origin of $k$-Schur functions was in a study of Macdonald polyomials where they are characterized as symmetric functions that depend on one additional parameter $t$. However, the bulk of our presentation lies in the $t=1$ setting. Although the general case is needed for implications in representation and Macdonald theory, the proven combinatorial and geometric properties largely center around this special case of $k$-Schur functions.

Extensive computer experimentation led to many conjectured properties of the $k$-Schur functions. Most notable is the $k$-Pieri rule for $k$-Schur functions, allowing one to express the product of a $k$-Schur function with a homogeneous symmetric function in terms of $k$-Schur functions. Chapter 2 starts by laying the combinatorial foundation needed to describe the $k$-Pieri rule including partitions, cores, and the affine Weyl group of type- $A$. Then, for fixed $k$ and for $t=1$, the $k$-Schur functions are presented as the family of symmetric functions which satisfy this Pieri rule. These functions form a basis of a subalgebra of the ring of symmetric functions. The dual basis lies in a Hopf-dual algebra which may be realized as a quotient of the ring of symmetric functions. Chapter 2 studies the $k$-Schur functions and their duals as symmetric functions, including a detailed summary of the weak and strong tableaux for which the $k$-Schur functions and their duals are the generating functions. Section 6 of this chapter includes an account of the affine insertion algorithm of [81], which explains how the generating functions for strong tableaux ( $k$-Schur functions) are known to be dual to the generating function for weak tableaux (dual $k$-Schur functions).

For arbitrary $t$, the $k$-Schur functions span a subspace of the ring of symmetric functions which is closed under the coproduct operation. It was in this setting that the $k$-Schur functions originally arose. They were first defined as a sum of the usual Schur functions over a combinatorially defined collection of tableaux known as a $k$-atom. Lapointe, Lascoux, and Morse [92] conjectured that the Macdonald symmetric functions expand positively in terms of $k$-Schur functions. An obvious difficulty with this approach is a missing algebraic connection that could be used to connect Macdonald symmetric functions with the combinatorics of $k$-atoms. A second definition of the $k$-Schur functions was given in terms of symmetric function operators and followed in subsequent research [93, 94]. Chapter 2, Section 3 discusses these definitions as well as several others that are conjecturally equivalent. Section 4 is used to give a list of mostly conjectural properties of $k$-Schur functions and an account of what is known (to date) about the status of these conjectures.

Throughout Chapter 2 we have included examples of computations with SAGE in order to demonstrate examples of the formulas, but also to show how to use the functions that have been written by developers and incorporated into SAGE. These examples will hopefully both inspire and encourage exploration so that readers can generate further data and make 
new conjectures about $k$-Schur functions and their duals.

Chapter 3 then goes into more depth about $k$-Schur functions in the setting of the nilCoxeter algebra. In the early 1980s, Stanley [150] became interested in the enumeration of the reduced words in the symmetric group. This led him to define a family of symmetric functions $\left\{F_{w} \mid w \in S_{n}\right\}$ now known as Stanley symmetric functions. In [78], Lam showed that the dual $k$-Schur functions were a special case of the affine Stanley symmetric functions $\tilde{F}_{w}$, analogues of Stanley's symmetric functions for the affine symmetric group.

In earlier work of Fomin and Stanley [48], it was shown that some of the main properties of Stanley symmetric functions could be obtained systematically from the nilCoxeter algebra of the symmetric group. This algebra is the associated graded algebra of the group algebra $\mathbb{C}\left[S_{n}\right]$ with respect to the length filtration. The affine nilCoxeter algebra played the same role for affine symmetric functions, and this provided an algebraic tool to study $k$-Schur functions and their duals. This interplay between algebra, combinatorics and symmetric functions is the main theme of Chapter 3. The connection to the nilHecke ring of Kostant and Kumar [75] is also explained and parts of the theory is carried out in the case of an arbitrary Weyl group.

Chapter 4 puts the preceding chapters in a more geometric context, and begins with a careful development of Kostant and Kumar's nilHecke ring $\mathbb{A}$ [75]. The nilHecke ring can roughly be described as the smash product of the nilCoxeter algebra and a polynomial ring and it was introduced to study the torus equivariant cohomology of Kac-Moody partial flag varieties. This ring acts as divided difference operators on the equivariant cohomology.

Peterson [131] studied the equivariant homology $H_{T}\left(\mathrm{Gr}_{G}\right)$ of the affine Grassmannian $\mathrm{Gr}_{G}$ of the complex simple simply-connected algebraic group $G$ as a Hopf algebra with the following idea: applying the homotopy equivalences $\mathrm{Gr} \simeq \Omega K$ and $\mathrm{Fl}_{\mathrm{af}} \simeq L K / T_{\mathbb{R}}$ the natural inclusion $\Omega K \hookrightarrow L K / T_{\mathbb{R}}$ gives rise to an action of $H_{T}\left(\mathrm{Gr}_{G}\right)$ on $H_{T}\left(\mathrm{Fl}_{\mathrm{af}}\right)$. (Here $\mathrm{Fl}_{\mathrm{af}}$ denotes the affine flag variety of $G$.) This action can be described in terms of divided difference operators, giving an injection $j: H_{T}\left(\mathrm{Gr}_{G}\right) \rightarrow \mathbb{A}$. Peterson's work is given a thorough treatment in Chapter 4, Section 4.

Using the natural relation between the nilCoxeter algebra and the nilHecke ring, in [78] Lam confirmed a conjecture of Morse and Shimozono identifying polynomial representatives for the Schubert classes of the affine Grassmannian as the $k$-Schur functions in homology and the dual $k$-Schur functions in cohomology. The algebraic part of this result is established in Chapter 3, Theorems 8.9 and 8.11.

We now discuss various generalizations of (dual) $k$-Schur functions, which are symmetricfunction versions of the Schubert bases of the dual Hopf algebras of the homology $H_{*}\left(\mathrm{Gr}_{S L_{n}}\right)$ and cohomology $H^{*}\left(\operatorname{Gr}_{S L_{n}}\right)$ of the type A affine Grassmannian $\operatorname{Gr}_{S L_{n}}$. For the affine Grassmannians $\operatorname{Gr}_{G}$ for $G$ of classical type, analogous symmetric functions have been defined [84, 132]. However, only for the analogues of dual $k$-Schur functions, is an explicit monomial expansion known. The classical type analogues of $k$-Schur functions are only defined by duality and little is known about their combinatorics.

There is an equivariant or "double" analogue of $k$-Schur functions, called $k$-double 
Schur functions [89], which are to $k$-Schur functions what double Schubert polynomials are to Schubert polynomials. These are symmetric functions for the Schubert bases of the equivariant homology $H_{T}\left(\mathrm{Gr}_{S L_{n}}\right)$ and $H^{T}\left(\mathrm{Gr}_{S L_{n}}\right)$ for the "small torus" $T$, a maximal torus in $G$ (as opposed to the maximal torus in the affine Kac-Moody group). The $k$ Schur functions are recovered from their double analogues by setting some variables to zero. Aside from setting up the correct symmetric function rings and bases, the only combinatorial result in this context is a Pieri rule for $H_{T}\left(\mathrm{Gr}_{S L_{n}}\right)$.

Essentially all of the general theory presented here has an analogue in $K$-theory, which carries more information than (co)homology. Passing from the $k$-Schur function to its $K$-theoretic analogue, is like passing from a Schubert polynomial to a Grothendieck polynomial. For the affine Grassmannian, as in (co)homology one again has a pair of dual Hopf algebras, but one obtains two pairs of dual bases; both algebras have a structure sheaf basis and an ideal sheaf basis. Kostant and Kumar developed the torus-equivariant $K$-theory of Kac-Moody homogeneous spaces [76] and Peterson's theory can be carried out in $K$-theory as well [85]. In particular Peterson's $j$-basis (see Chapter 4, Section 4.5), which is defined algebraically using a leading term condition for an expansion in the divided difference basis, has an analogue (called the $k$-basis in [85]) that corresponds to ideal sheaves of Schubert varieties in the affine Grassmannian. Peterson's "quantum equals affine" theorem $[131,88]$ (see Chapter 4, Section 4.7) has an analogue in $K$-theory: the structure sheaves of opposite Schubert varieties in the quantum $K$-theory $Q K^{T}(G / B)$ of finite-dimensional flag varieties $G / B$, appear to multiply in the same way as the structure sheaves of the Schubert varieties in the $K$-homology $K_{T}\left(\mathrm{Gr}_{G}\right)$ of the affine Grassmannian [83]. To establish this connection one must prove a conjectural Chevalley formula of Lenart and Postnikov [114, Conjecture 17.1] for quantum K-theory.

\section{Acknowledgements}

This material is based upon work supported by the National Science Foundation under Grant No. DMS-0652641. "Any opinions, findings, and conclusions or recommendations expressed in this material are those of the author(s) and do not necessarily reflect the views of the National Science Foundation." We are grateful to the Fields Institute in Toronto for helping organize and support the summer school and workshop on "Affine Schubert calculus".

We would like to thank Tom Denton and Karola Mészáros for helpful comments and additions on Chapter 2, and Jason Bandlow, Chris Berg, Nicolas M. Thiéry as well as many other SAGE developers for their help with the SAGE implementations.

\section{A Appendix: SAGE}

SAGE [152] is a completely open source general purpose mathematical software system, 
which appeared under the leadership of William Stein (University of Washington) and has developed explosively within the last five years. It is similar to MAPLE, MUPAD, MATHEmatica, Magma, and up to some point Matlab, and is based on the popular Python programming language. SAGE has gained strong momentum in the mathematics community far beyond its initial focus in number theory, in particular in the field of combinatorics, see [141].

Tutorials and instructions on how to install SAGE can be found at the main SAGE website http://www.sagemath.org/. For example, for the basic SAGE syntax and programming tricks see http://www.sagemath.org/doc/tutorial/programming.html.

Many aspects related to $k$-Schur functions and symmetric functions in general have been implemented in SAGE and in fact are still being developed as an on-going project. Throughout the text we provide many examples on how to use SAGE to do calculations related to $k$-Schur functions. Further information about the latest code and developments can be obtained from the SAgE-Combinat website [141]. 


\title{
Chapter 2
}

\section{Primer on $k$-Schur Functions}

\author{
Jennifer Morse $^{1}$, Anne Schilling ${ }^{2}$ And Mike Zabrocki ${ }^{3}$ \\ morsej@math.drexel.edu, anne@math.ucdavis.edu, zabrocki@mathstat.yorku.ca \\ based on lectures by Luc Lapointe and Jennifer Morse \\ lapointe@inst-mat.utalca.cl and morsej@math.drexel.edu
}

The purpose of this chapter is to outline some of the results and open problems related to $k$-Schur functions, mostly in the setting of symmetric function theory. This chapter roughly follows the outline of several talks given by Luc Lapointe and Jennifer Morse at a conference titled "Affine Schubert Calculus" held in July of 2010 at the Fields Institute in Toronto ${ }^{4}$.

In addition it presents many examples based on code written in SAGE $[152,141]$ by Jason Bandlow, Nicolas M. Thiéry, the last two authors, and many other SAGE developers. The following presentation is intended to give both an idea of the origins of the $k$-Schur functions as well as the current ideas and computational tools which have been most productive for demonstrating their properties.

We will present almost no proofs in this chapter, but rather refer to the original articles for detailed arguments. Instead the concepts are illustrated with many SAGE examples to highlight how to discover and experiment with many of the still open conjectures related to $k$-Schur functions. The purpose behind most of the SAGE examples is to demonstrate the formulas with examples and to give the commands that would allow a first time user of SAGE to be able to use the functions to generate data that they might need for their own research.

\footnotetext{
${ }^{1}$ The author was supported by NSF gant DMS-0652641, DMS-0652668, DMS-1001898.

${ }^{2}$ The author was supported by NSF grants DMS-0652641, DMS-0652652, DMS-1001256, and OCI1147247.

${ }^{3}$ The author was supported by NSERC

${ }^{4}$ see http://www.fields.utoronto.ca/programs/scientific/10-11/schubert/
} 
Section 1 reviews much of the combinatorial background of $k$-Schur theory including partitions, cores, (partial) orders on the affine symmetric group, and some symmetric function theory. This section also sets up the combinatorial backdrop needed to give the Pieri rules for $k$-Schur functions and their duals. In Section 2 , we define a parameterless $(t=$ 1) family of $k$-Schur functions using an analogue of the Pieri rule for Schur functions [95]. This definition is used to relate $k$-Schur functions to the geometry and to Stanley symmetric functions discussed in Chapter 3. We also give the dual Pieri rule [81] which gives rise to a monomial expansion of the $k$-Schur functions. The Pieri and dual Pieri rule motivate the definition of weak and strong order tableaux.

In Section 3, we present four conjecturally equivalent definitions of the $k$-Schur functions for generic $t$. Some are known to be equivalent when $t=1$. The first definition of $k$ Schur functions appeared in a paper by Lapointe, Lascoux and Morse [92] and is purely combinatorial in nature; defined as a sum over certain classes of tableaux called atoms. Lapointe and Morse [93] followed this paper by defining symmetric functions which were defined by algebraic operations instead of a sum over combinatorial objects. The last two definitions of the $k$-Schur functions with a generic parameter $t$ are defined along lines similar to the parameterless $k$-Schur functions, but now a $t$-statistic is introduced on weak (resp. strong) order tableaux.

In Section 4 we present many of the properties of $k$-Schur functions and outline what is known about which property for each of the definitions. This is followed by Section 5 which contains further research directions and many conjectures that remain to be resolved (and hence the content is likely to change in the future)! Section 6 explains the duality between strong and weak order in terms of a $k$-analogue of the Robinson-Schensted-Knuth algorithm, which gives rise to an affine insertion algorithm. We present part of this algorithm by giving a bijection between permutations and pairs of tableaux. Finally in Section 7 some details about the branching from $k$ to $(k+1)$-Schur functions are given.

\section{Background and notation}

\subsection{Partitions and cores}

A partition $\lambda=\left(\lambda_{1}, \lambda_{2}, \ldots, \lambda_{\ell(\lambda)}\right)$ of $m$ is a sequence of weakly decreasing positive integers which sum to $m=\lambda_{1}+\lambda_{2}+\cdots+\lambda_{\ell(\lambda)}$. The value of $m$ is called the size of the partition and this will be denoted by $|\lambda|$. The entries of the partition are called the parts and the number of parts of the partition is denoted by $\ell(\lambda)$. As a general convention, if $i>\ell(\lambda)$ then $\lambda_{i}=0$ and the definition of symmetric functions (which turn out to be indexed by partitions) given later in this section respects this convention. The statistic $n(\lambda)=\sum_{i=1}^{\ell(\lambda)}(i-1) \lambda_{i}$ on partitions has a value between 0 and $m(m-1) / 2$ for partitions of $m$ and this will arise in the definitions of symmetric functions. A partition $\lambda$ is called $k$-bounded if $\lambda_{1} \leq k$. The notation $\lambda \vdash m$ indicates that $\lambda$ is a partition of $m$ and generally we reserve the symbols $\lambda, \mu, \nu$ to denote partitions. 
A partition will be identified with its Young (or Ferrers) diagram. This is a diagram consisting of square cells arranged in left justified rows stacked on top of each other with the largest row with $\lambda_{1}$ cells on the bottom. (This convention is also called the French notation; when stacking the rows with the largest row at the top is called the English convention). Alternatively, a Young diagram is a collection of cells in the first quadrant of the $(x, y)$-plane with $\operatorname{dg}(\lambda)=\left\{(i, j): 1 \leq i \leq \ell(\lambda)\right.$ and $\left.1 \leq j \leq \lambda_{i}\right\}$ represented as boxes in the Cartesian plane so that the upper right hand corner of a cell has coordinate which is in this collection. For consistency with other references we have chosen that the first coordinate represents the row and the second coordinate represents the column (each beginning at 1 for the first row and column). For an example the Young diagram for the partition $\lambda=(4,3,3,3,2,2,1)$ is drawn in Example 1.1.

There is a partial order on partitions that arises naturally in symmetric functions when ordering basis elements. For two partitions $\lambda, \mu$ such that $|\lambda|=|\mu|$, we say that $\lambda \leq \mu$ if $\sum_{i=1}^{r} \lambda_{i} \leq \sum_{i=1}^{r} \mu_{i}$ for all $r \geq 1$. This is usually referred to as the dominance order on partitions.

The conjugate of a partition $\lambda$ is the sequence $\lambda^{\prime}=\left(\lambda_{1}^{\prime}, \lambda_{2}^{\prime}, \ldots, \lambda_{\lambda_{1}}^{\prime}\right)$ where $\lambda_{r}^{\prime}=\#\{i$ : $\left.\lambda_{i} \geq r\right\}$. Alternatively, this can be seen on Young diagrams by reflecting the diagram in the $x=y$ line of the coordinate plane so that $\operatorname{dg}\left(\lambda^{\prime}\right)=\{(j, i):(i, j) \in \operatorname{dg}(\lambda)\}$. For example in Example 1.1 below, $\lambda^{\prime}=(7,6,4,1)$ for the partition $\lambda=(4,3,3,3,2,2,1)$.

For many uses we will need to refer to the number of parts of a partition of a given size $i$ and this will be denoted by $m_{i}(\lambda)=\#\left\{j: \lambda_{j}=i\right\}$. The quantity

$$
z_{\lambda}=\prod_{i \geq 1} m_{i}(\lambda) ! i^{m_{i}(\lambda)}
$$

is the size of the stabilizer of a permutation $\sigma \in S_{m}$, the symmetric group on $m=|\lambda|$ letters, whose cycle type is $\lambda$ under the conjugation action of $S_{m}$. That is, if $\sigma$ has cycle type $\lambda$, then $z_{\lambda}=\#\left\{\tau \in S_{m}: \tau \sigma \tau^{-1}=\sigma\right\}$. Since we know that all permutations with the same cycle type are conjugate, the number of permutations with cycle type $\lambda$ is equal to $m ! / z_{\lambda}$.

Each cell in a partition $\lambda$ has a hook length which consists of the number of cells in the column above and in the row to the right (including the cell itself). Namely, for a cell $(i, j) \in \operatorname{dg}(\lambda)$, the hook length of the cell is $\operatorname{hook}_{\lambda}(i, j)=\lambda_{i}+\lambda_{j}^{\prime}-i-j+1$. In Example 1.1

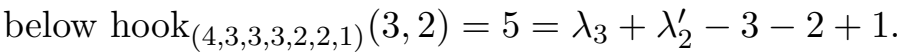

For a partition $\lambda$ with $\lambda_{1} \leq k$, define the $k$-split of $\lambda$ as a sequence of partitions (which will be denoted by $\lambda^{\rightarrow k}$ ) recursively. If $\lambda_{1}+\ell(\lambda)-1 \leq k$, then $\lambda^{\rightarrow k}=(\lambda)$. Otherwise,

$$
\lambda^{\rightarrow k}=\left(\left(\lambda_{1}, \lambda_{2}, \ldots, \lambda_{k-\lambda_{1}+1}\right),\left(\lambda_{k-\lambda_{1}+2}, \lambda_{k-\lambda_{1}+3}, \ldots, \lambda_{\ell(\lambda)}\right)^{\rightarrow k}\right) .
$$

In other words, the $k$-split of a partition is found by successively splitting off parts of the partition with hook $k$, starting with the first part, until that is no longer possible. 
Example 1.1. The Young diagram for the partition $\lambda=(4,3,3,3,2,2,1)$ is the diagram on the left and its conjugate partition $\lambda^{\prime}=(7,6,4,1)$ is the diagram in the center.
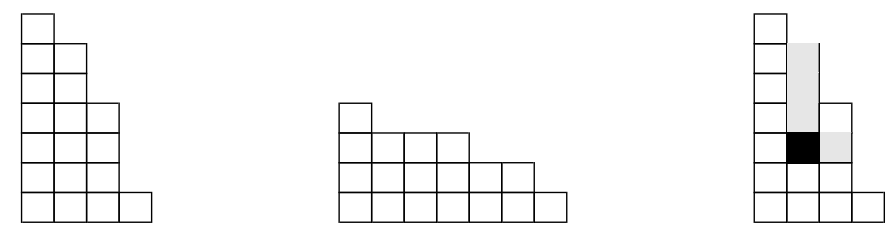

The diagram on the right is the Young diagram for the partition $\lambda=(4,3,3,3,2,2,1)$ with the cells that are in the hook of the cell $(3,2)$ shaded in. In this case $\operatorname{hook}_{\lambda}(3,2)=5$. The 4 -split of $\lambda$ is $\lambda^{\rightarrow 4}=((4),(3,3),(3,2),(2,1))$ and the 5 -split is $\lambda^{\rightarrow 5}=((4,3),(3,3,2),(2,1))$.

We will use the realization of the Young diagram as the set of cells in our notation and define $\lambda \subseteq \mu$ if $\operatorname{dg}(\lambda) \subseteq \operatorname{dg}(\mu)$. This forms a lattice, also known as the Young lattice, on set of partitions and the cover relation is given by $\lambda \rightarrow \mu$ if $\lambda \subseteq \mu$ and $|\lambda|+1=|\mu|$. The lattice is graded by the size of the partition and the first 6 levels of the infinite Hasse diagram are shown in Figure 2.1.

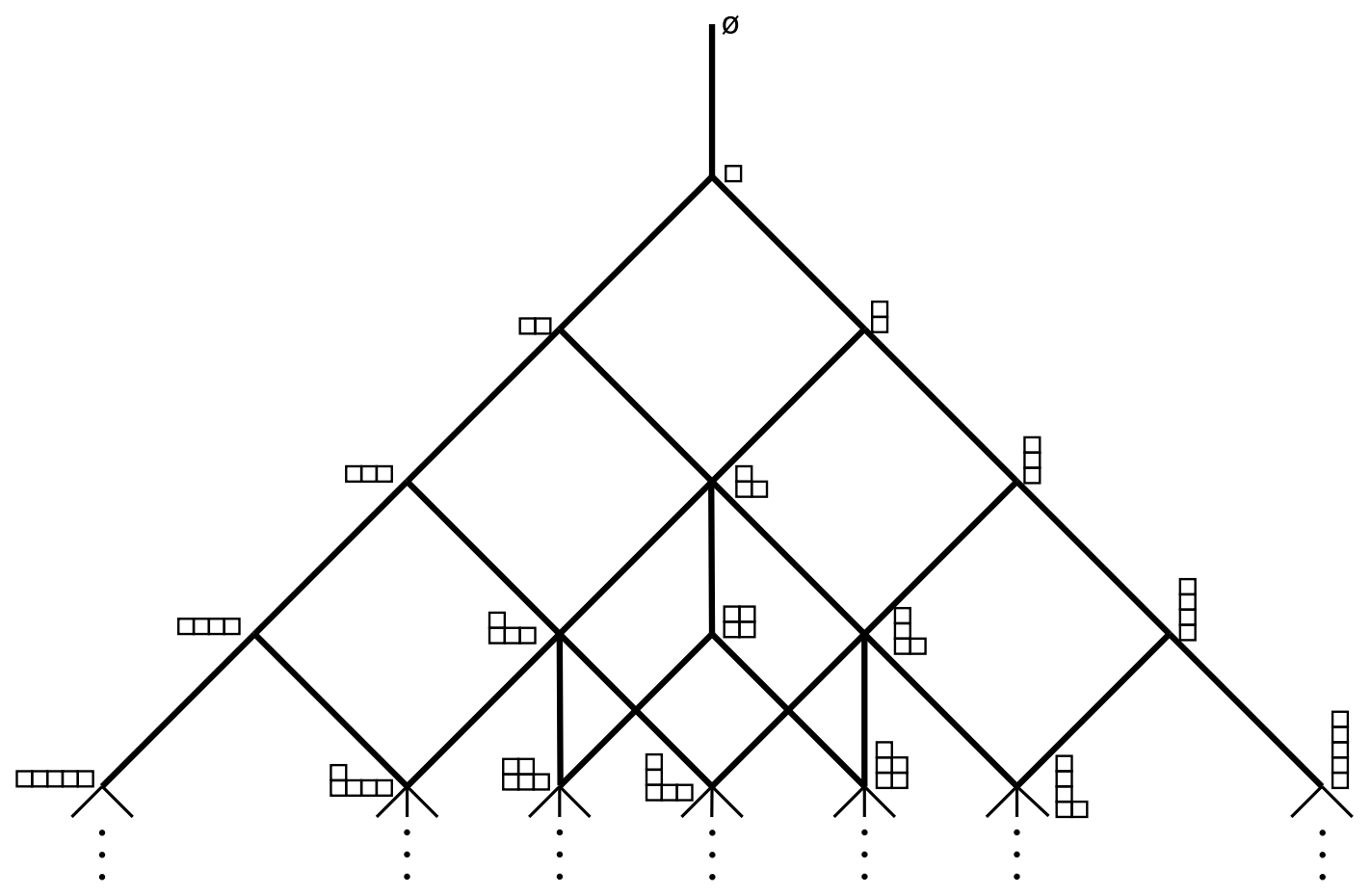

Figure 2.1: The Young lattice of partitions (up to those of size 5) ordered by inclusion . 
There are several special types of containments of partitions that will arise in this discussion. If $\lambda \subseteq \mu$, then $\mu / \lambda$ is called a skew partition and it will represent the cells which are in $\operatorname{dg}(\mu) / \operatorname{dg}(\lambda)$, with the / here representing the difference of sets. We call $\mu / \lambda$ connected if for any two cells there is a sequence of cells in $\mu / \lambda$ from one to the other where consecutive cells share an edge. We say that $\mu / \lambda$ is a horizontal (vertical) strip if there is at most one cell in each column (row) of $\mu / \lambda$. The skew partition $\mu / \lambda$ is called a ribbon if it does not contain any $2 \times 2$ subset of cells.

Sage Example 1.2. We now demonstrate how to access partitions and their properties in the open source computer algebra system SAGE (see Appendix A). We begin by listing all partitions of 4 :

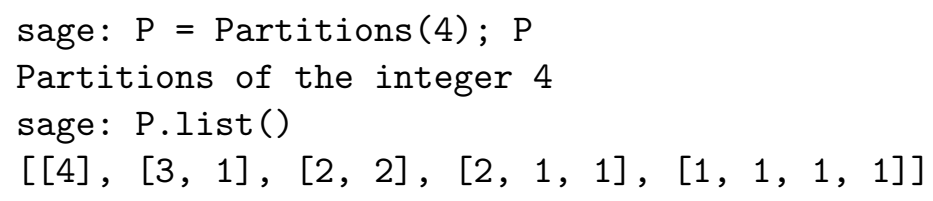

SAGE has list comprehension so that the last line could have also been written as

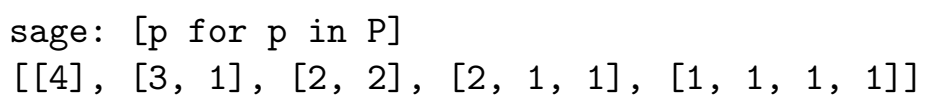

We can check how two partitions $\lambda$ and $\mu$ relate in the dominance order

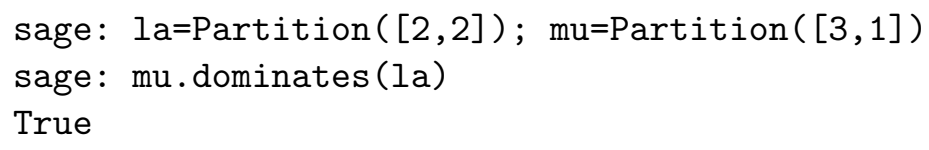

and draw the entire Hasse diagram

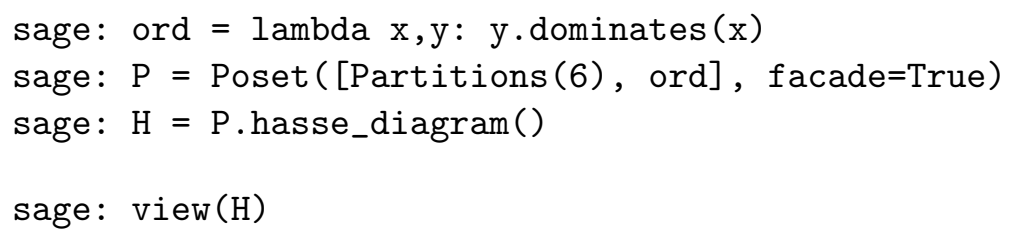

which outputs the graph. Here we used the python syntax for a function, which is lambda $\mathrm{x}: \mathrm{f}(\mathrm{x})$ for a function that maps $x$ to $f(x)$. We can also compute the conjugate of a partition, its $k$-split

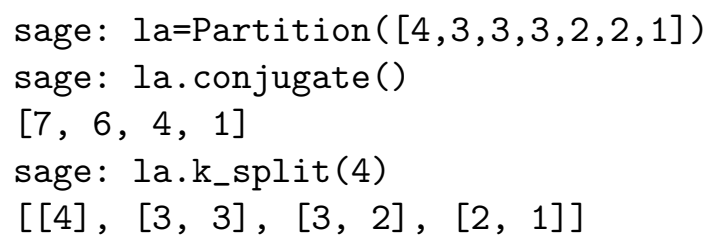


and create skew partitions

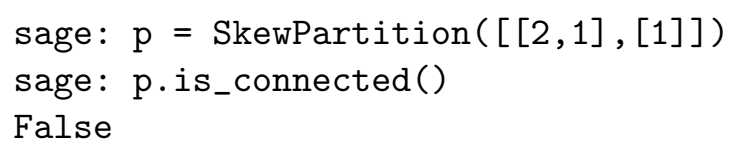

\subsection{Bounded partitions, cores, and affine Grassmannian elements}

We will see that $k$-Schur functions are symmetric functions indexed by $k$-bounded partitions and consequently, the underlying combinatorial framework we need often comes out of a refinement of classical ideas in the theory of partitions. As it happens, the set of $k$-bounded partitions is in bijection with several different sets of natural combinatorial objects and often the $k$-Schur function setting is better expressed in those terms. To this end, we begin with a discussion of several other examples of possible indexing sets.

As with the $k$-bounded partitions, we are interested in another special subset of partitions. In particular, an $r$-core is a shape where none of its cells have a hook-length equal to $r$. We denote the set of all $r$-cores by $\mathcal{C}_{r}$. When we consider a partition as a core, the notion of size differs from the usual notion (where size counts the number of cells in the shape). In contrast, the relevant notion of size on a $(k+1)$-core is to count only the number of cells which have a hook-length smaller than $k+1$. We call this the length of the core. For a $(k+1)$-core $\kappa$, its length will be denoted by $|\kappa|_{k+1}$ or simply $|\kappa|$ if it is clear from the context that $\kappa$ is viewed as a $(k+1)$-core. As $k \rightarrow \infty$, this becomes the usual size of the partition. Later in this section, we will see that the length is related to the length of elements in the affine symmetric group. Now, we give the connection between cores and bounded partitions.

Proposition 1.3. ([95, Theorem 7]) There is a bijection between the set of $(k+1)$-cores $\kappa$ with $|\kappa|_{k+1}=m$ and partitions $\lambda \vdash m$ with $\lambda_{1} \leq k$.

The bijection from $(k+1)$-cores to $k$-bounded partitions is

$$
\mathfrak{p}: \kappa \mapsto \lambda,
$$

defined by setting

$$
\lambda_{i}=\#\left\{(i, j) \in \kappa: \operatorname{hook}_{\kappa}(i, j) \leq k\right\} .
$$

Example 1.4. The partition $(12,8,5,5,2,2,1)$ on the left is a 5 -core since there are no cells in its Ferrers diagram with hook-length equal to 5. Equation (1.3) tells us how to applying $\mathfrak{p}$ to this core to obtain a 4-bounded partition; delete each cell in the diagram for the 5 -core whose hook-length exceeds 5 and then slide all remaining cells to the left.
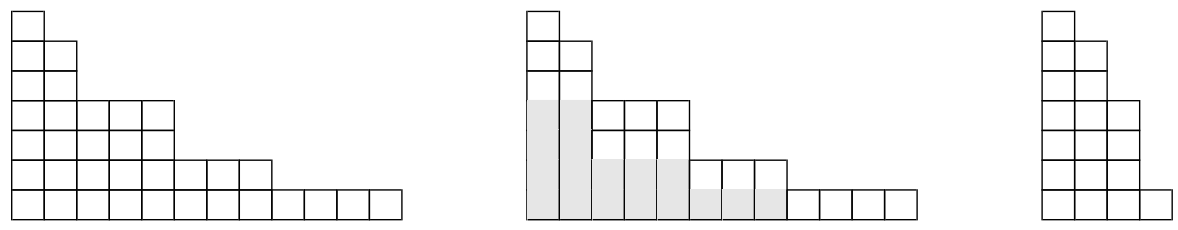
The first part of the resulting partition is at most 4 .

The other direction of the bijection is also not difficult. Consider a $k$-bounded partition and work from the smallest part of the partition to the largest and slide the cells to the right until it is a $(k+1)$-core. Here is a description of the procedure which can be followed with Example 1.5. Start with the top row $\lambda_{\ell(\lambda)}$ of the $k$-bounded partition $\lambda$ and successively move down a row. For a given row, calculate the hook lengths of its cells; if there is a cell with hook length greater than $k$, slide this row to the right until all cells have hook length less than or equal to $k$. Continue this process until all rows have been adjusted. The end result will be a $(k+1)$-core which we shall denote by $\mathfrak{c}_{k}(\lambda)$ or just $\mathfrak{c}(\lambda)$ if $k$ is clear from the context.

Example 1.5. The partition $(4,3,3,3,2,2,1)$ is a 4-bounded partition. Here we draw the successive slides of the rows until we reach a 5 -core:

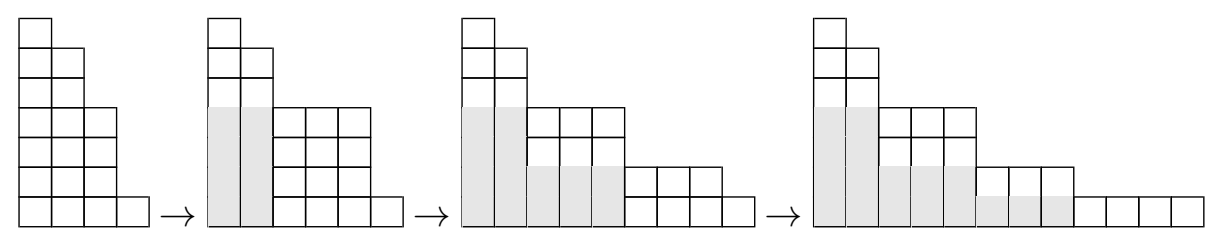

Sage Example 1.6. Here is the way to compute the map $\mathfrak{c}$ in SAGE:

$$
\begin{aligned}
& \text { sage: } l a=\operatorname{Partition}([4,3,3,3,2,2,1]) \\
& \text { sage: kappa }=\text { la.k_skew }(4) ; \mathrm{kappa} \\
& {[12,8,5,5,2,2,1] /[8,5,2,2]}
\end{aligned}
$$

For the inverse $\mathfrak{p}$ we write

sage: kappa.row_lengths ()

$[4,3,3,3,2,2,1]$

If one is only handed the 5 -core $(12,8,5,5,2,2,1)$ instead of the skew partition, one can do the following:

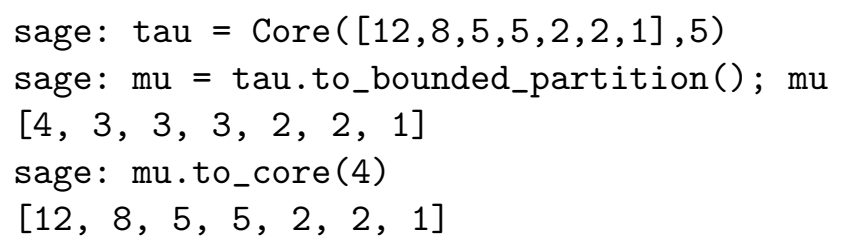

All 3-cores of length 6 can be listed as:

sage: $\operatorname{Cores}(3,6)$.list ()

$[[6,4,2],[5,3,1,1],[4,2,2,1,1],[3,3,2,2,1,1]]$ 
We now turn our attention to the third set of objects that is in bijection with the set of $k$-bounded partitions (and the set of $(k+1)$-cores). These come out of studying the type $A$ affine Weyl group and its realization as the affine symmetric group $\tilde{S}_{n}$ given by generators $\left\{s_{0}, s_{1}, \ldots, s_{n-1}\right\}$ satisfying the relations

$$
\begin{aligned}
& s_{i}^{2}=1, \\
& s_{i} s_{i+1} s_{i}=s_{i+1} s_{i} s_{i+1}, \\
& s_{i} s_{j}=s_{j} s_{i} \quad \text { for } i-j \not \equiv \quad 0,1, n-1 \quad(\bmod n)
\end{aligned}
$$

with all indices related $(\bmod n)$. Hereafter, we shall reserve parameters $n$ and $k$ and we will set $n=k+1$ throughout.

There is a subset of the elements in $\tilde{S}_{n}$ that is particularly conducive to combinatorics in large part because it is in bijection with the set of $k$-bounded partitions and of $(k+1)$ cores. Note that the symmetric group $S_{n}$ generated by $\left\{s_{1}, s_{2}, \ldots, s_{n-1}\right\}$ is a subgroup, where the element $s_{i}$ represents the permutation which interchanges $i$ and $i+1$. We will refer to the left cosets of $\tilde{S}_{n} / S_{n}$ as affine Grassmannian elements and they will be identified with their minimal length coset representatives, that is, the elements of $w \in \tilde{S}_{n}$ such that either $w=i d$ or $s_{0}$ is the only elementary transposition such that $\ell\left(w s_{0}\right)<\ell(w)$.

Remark 1.7. The definition of affine Grassmannian elements are the special case of a more general definition. The $l$-Grassmannian elements are the minimal length coset representatives of $\tilde{S}_{n} / S_{n}^{l}$ where $S_{n}^{l}$ is the group generated by $\left\{s_{0}, s_{1}, s_{2}, \ldots, s_{n-1}\right\} \backslash\left\{s_{l}\right\}$ and the affine Grassmannian elements are the 0-Grassmannian elements. Due to the cyclic symmetry of the affine type $A$ Dynkin diagram, these constructions are of course all equivalent.

Sage Example 1.8. We can create the affine symmetric group and its generators in SAGE as

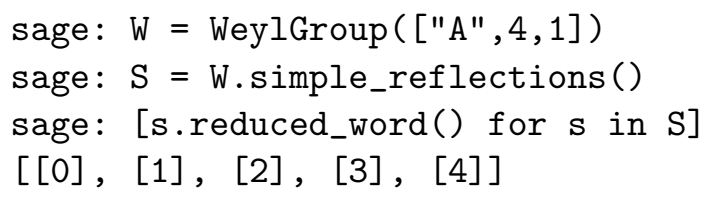

For a given element, we can ask for its reduced word or create it from a word in the generators and ask whether it is Grassmannian:

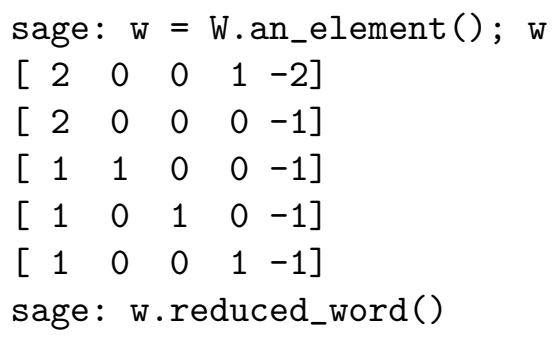




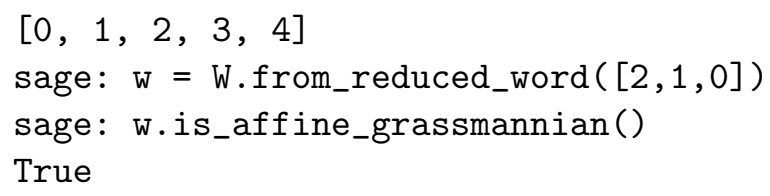

Proposition 1.9. [103] [95, Proposition 40] There is a bijection between the collection of cosets $\tilde{S}_{k+1} / S_{k+1}$ whose minimal length representative has length $m$ and $(k+1)$-cores of length $m$.

The bijection of Proposition 1.9 is defined by an action of the affine symmetric group on cores. It suffices to define the left action of the generators $s_{i}$ of the affine symmetric group on $(k+1)$-cores. The diagonal index or content of a cell $c=(i, j)$ in the diagram for a core is $j-i$. We will often instead be concerned with the residue of $c$, denoted by $\operatorname{res}(c)$, which is the diagonal index $\bmod k+1$. We call a cell $c$ an addable corner of a partition $\mu$ if $\operatorname{dg}(\mu) \cup\{c\}$ is the diagram for a partition and a cell $c$ is a removable corner of $\mu$ if $\operatorname{dg}(\mu) \backslash\{c\}$ is diagram for a partition.

Definition 1.10. [103] [95, Definition 18] For $\kappa$ a $(k+1)$-core, let $s_{i} \cdot \kappa$ be the partition with

1. if there is at least one addable corner of residue $i$, then the result is $\kappa$ with all addable corners of $\kappa$ of residue $i$ added,

2. if there is at least one removable corner of residue $i$, then the result is $\kappa$ with all removable corners of $\kappa$ of residue $i$ removed,

3. otherwise, the result is $\kappa$.

Example 1.11. Consider the 5-core, $(7,3,1)$. If we draw its Ferrers diagrams and label each of the cells with the content modulo 5 we have the following diagram

\begin{tabular}{|c|c|c|c|}
\hline \multicolumn{4}{|c|}{3} \\
\hline & 0 & & \\
\hline \begin{tabular}{|l|l}
0 \\
\end{tabular} & 1 & 2 & \begin{tabular}{l|l|l|l|}
3 & 4 & 0 & 1 \\
\end{tabular} \\
\hline
\end{tabular}

This diagram has addable corners with residue 2 and 4 and removable corners with residue 1 and 3 and all cells of residue 0 are neither addable nor removable. Therefore, $s_{2}$. $(7,3,1)=(8,4,1,1), s_{4} \cdot(7,3,1)=(7,3,2), s_{1} \cdot(7,3,1)=(6,2,1), s_{3} \cdot(7,3,1)=(7,3)$, $s_{0} \cdot(7,3,1)=(7,3,1)$.

Sage Example 1.12. In SAGE we can get the affine symmetric group action on cores as follows: 


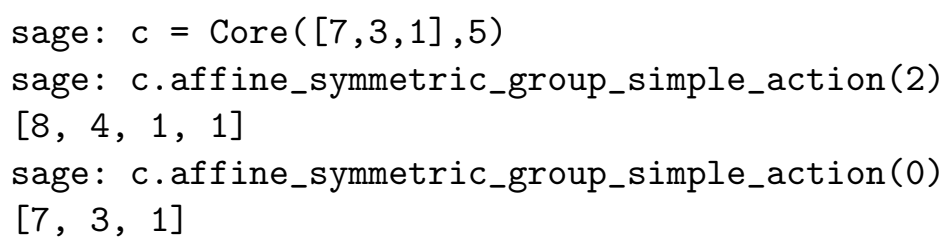

We can also check directly that the set of affine Grassmannian elements of given length are in bijection with the corresponding cores:

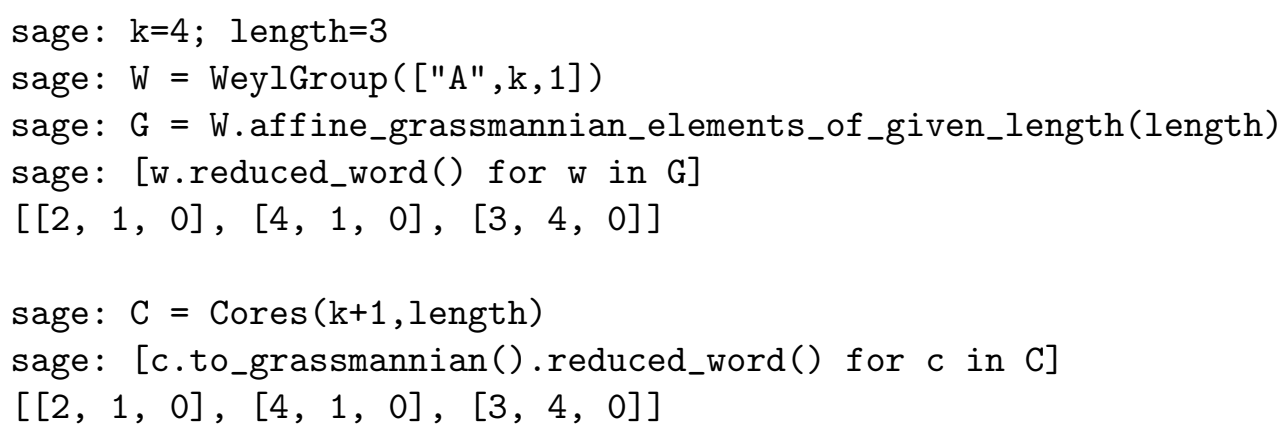

The bijection of Proposition 1.9 is realized by taking a reduced word for an affine Grassmannian element and acting on the empty $(k+1)$-core. The resulting $(k+1)$-core is the image of the bijection. [95, Corollary 48] then states that the reverse bijection can be found by taking $\lambda=\mathfrak{p}(\kappa)$ and forming the element in the affine symmetric group $s_{\text {res }\left(c_{1}\right)} s_{\text {res }\left(c_{2}\right)} \cdots s_{\text {res }\left(c_{m}\right)}$, where $c_{1}, c_{2}, \ldots, c_{m}$ are the cells of $\operatorname{dg}(\lambda)$ read from the smallest row to the largest with each read from right to left.

We denote the map which sends $(k+1)$-core $\kappa$ to the corresponding affine Grassmannian element by $\mathfrak{a}(\kappa)=w_{\kappa}$. Since $(k+1)$-cores and $k$-bounded partitions are in bijection, we will also use the notation $\mathfrak{a}(\lambda)=w_{\lambda}$ to represent the map from a $k$-bounded partition $\lambda$ to an affine Grassmannian element.

Example 1.13. Consider the reduced word,

$$
w=s_{1} s_{0} s_{4} s_{2} s_{3} s_{1} s_{0} s_{4} s_{1} s_{2} s_{3} s_{1} s_{0} s_{4} s_{3} s_{2} s_{1} s_{0}
$$

We apply this word on the left on an empty 5 -core to build up the result. The sequence of applications builds the core as follows:

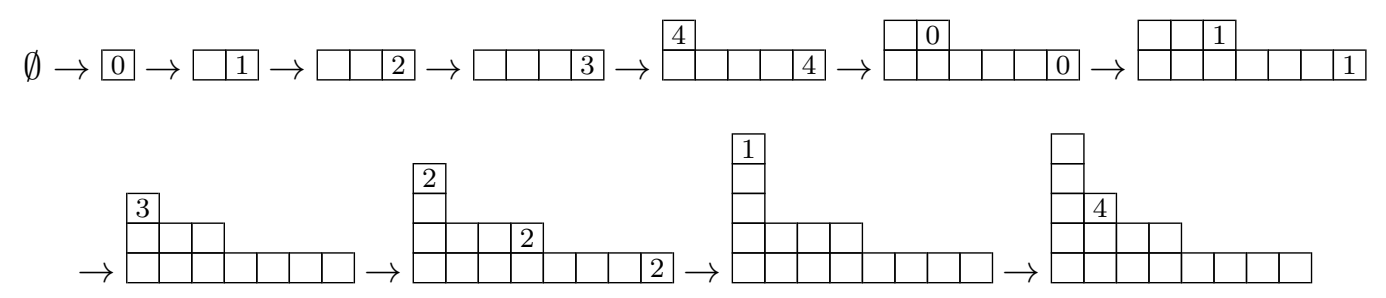



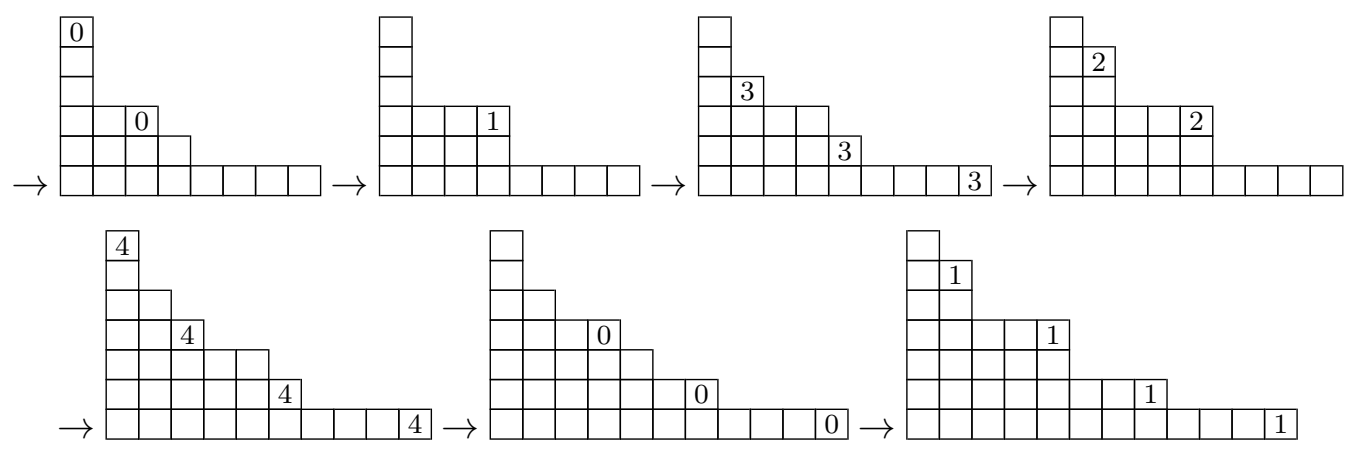

and hence the resulting 5 -core is $\mathfrak{a}^{-1}(w)=(12,8,5,5,2,2,1)$.

The reverse bijection comes from reading the residues of the corresponding 4-bounded partition $(4,3,3,3,2,2,1)$ from the smallest row to the largest row, and from right to left within the rows. For example:

\begin{tabular}{|c|c|c|}
\hline 4 & & \\
\hline$\underline{0}$ & 1 & \\
\hline 1 & 2 & \\
\hline \begin{tabular}{|l}
2 \\
\end{tabular} & 3 & 4 \\
\hline \begin{tabular}{|l|}
3 \\
\end{tabular} & 4 & 0 \\
\hline 4 & 0 & 1 \\
\hline 0 & & 2 \\
\hline
\end{tabular}

is sent under $\mathfrak{a}$ to the word

$$
w^{\prime}=s_{4} s_{1} s_{0} s_{2} s_{1} s_{4} s_{3} s_{2} s_{0} s_{4} s_{3} s_{1} s_{0} s_{4} s_{3} s_{2} s_{1} s_{0} .
$$

It is not difficult to show that $w$ is equivalent to $w^{\prime}$.

Sage Example 1.14. We can verify the previous example in SAGE.

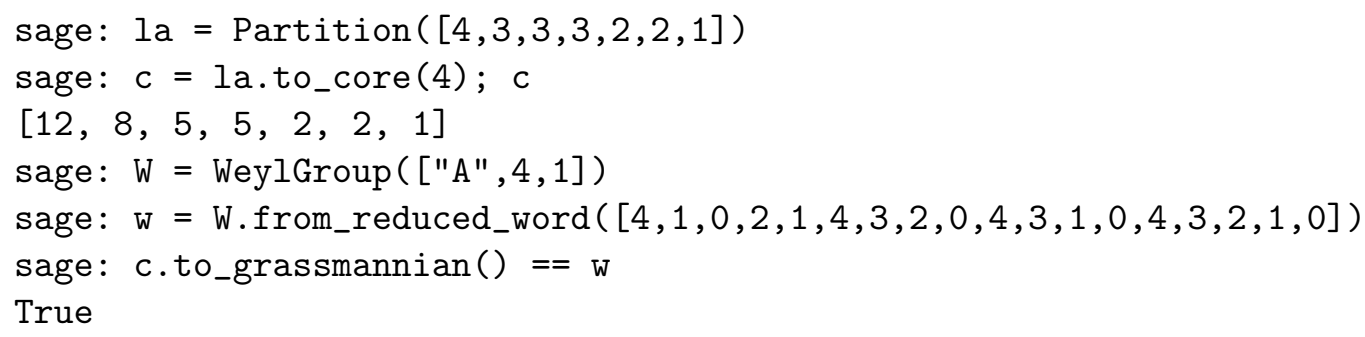

The affine symmetric group $\tilde{S}_{n}$ can also be thought of as the group of permutations of $\mathbb{Z}$ with the property that for $w \in \tilde{S}_{n}$ we have $w(i+r n)=w(i)+r n$ for all $r \in \mathbb{Z}$ with the additional property that $\sum_{i=1}^{n} w(i)-i=0$. We can choose the convention that the elements $s_{i} \in \tilde{S}_{n}$ for $0 \leq i \leq n-1$ act on $\mathbb{Z}$ by $s_{i}(i+r n)=i+1+r n, s_{i}(i+1+r n)=i+r n$, and $s_{i}(j)=j$ for $j \not \equiv \quad i, i+1(\bmod n)$. While the elements $s_{i}$ generate the group, there is also the notion of a general transposition $t_{i j}$ which generalizes this notion by interchanging 
$i$ and $j(\bmod n)$. Take integers $i<j$ with $i \not \equiv \quad j(\bmod n)$ and $v=\lfloor(j-i) / n\rfloor$, then $t_{i, i+1}=s_{i}$ and for $j-i>1$,

$$
t_{i j}=s_{i} s_{i+1} s_{i+2} \cdots s_{j-v-2} s_{j-v-1} s_{j-v-2} s_{j-v-3} \cdots s_{i+1} s_{i}
$$

where all of the indices of the $s_{m}$ are taken $(\bmod n)$. For $j>i$, we set $t_{i j}=t_{j i}$. The $t_{i j}$ generalize the elements $s_{i}$ by their action $t_{i j}(i+r n)=j+r n, t_{i j}(j+r n)=i+r n$ and $t_{i j}(\ell)=\ell$ for $\ell \not \equiv i, j(\bmod n)$. It is not hard to show that

$$
t_{i j} w=w t_{w^{-1}(i) w^{-1}(j)}
$$

which allows us to define a left as well as a right action on affine permutations. Here we state the results in terms of the left action.

The elements $w \in \tilde{S}_{n}$ are determined by the action of $w$ on the values 1 through $n$ since this determines the action on all of $\mathbb{Z}$ by $w(i+r n)=w(i)+r n$. If $w$ is represented in two line notation,

$$
\begin{array}{ccccccc}
\cdots & -2 & -1 & 0 & 1 & 2 & \cdots \\
\cdots & w(-2) & w(-1) & w(0) & w(1) & w(2) & \cdots
\end{array}
$$

then $t_{i j} w$ is obtained from $w$ by exchanging $i+r n$ and $j+r n$ in the lower row of the two line notation for $w$. We also have that $w t_{i j}$ is obtained from $w$ by exchanging $w(i+r n)$ and $w(j+r n)$. An element is affine Grassmannian if $w(1)<w(2)<\cdots<w(n)$. The tuple of values $[w(1), w(2), \ldots, w(n)]$ is referred to as the window notation for $w$.

There is a close relationship between the action of $w^{-1}$ on integers and on cores. This relationship is best demonstrated by an example before we give the precise statement.

Example 1.15. Start with $n=k+1=3$ and the affine Grassmannian element $w=$ $s_{1} s_{0} s_{2} s_{1} s_{0}$ that has window notation $[w(1), w(2), w(3)]=[-2,0,8]$. We can use this to determine that $w^{-1}$ is given in two line notation as

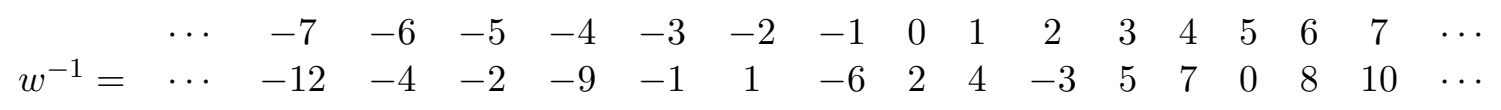

Now we notice in this notation that $w^{-1}(d) \leq 0$ for all integers $d \leq-3$ (and for any affine permutation there will always be an integer $d^{\prime}$ such that $w^{-1}(d) \leq 0$ for all $\left.d \leq d^{\prime}\right)$. We also see that for all integers $d \geq 6$ we have $w^{-1}(d)>0$ (and for any affine permutation there will always be a value $D^{\prime}$ such that $w^{-1}(d)>0$ for all $\left.d \geq D^{\prime}\right)$.

Now consider the integers $-2 \leq d \leq 5$ (which are the integers strictly between these two values $d^{\prime}=-3$ and $D^{\prime}=6$ ). Reading from left to right in the two line notation, we construct a path consisting of East and South steps where for each $d$ such that $w^{-1}(d) \leq 0$ we place a South step, and for each $d$ such that $w^{-1}(d)>0$ we place an East step. In this example we are looking at the sequence 


$\begin{array}{cccccccc}-2 & -1 & 0 & 1 & 2 & 3 & 4 & 5 \\ 1 & -6 & 2 & 4 & -3 & 5 & 7 & 0\end{array}$

in order to create this path. The way we have chosen our $d^{\prime}$ and $D^{\prime}$ the first step of this path will always be East and the last step will always be South. In this example we have the path:

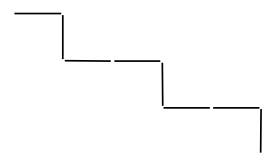

and it is the outline for the diagram of a 3-core:

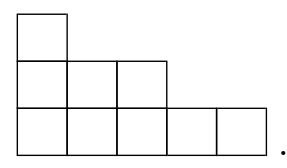

It is the case that $\mathfrak{c}(2,2,1)=(5,3,1)$ and corresponds to the affine permutations $w=$ $s_{1} s_{0} s_{2} s_{1} s_{0}=\mathfrak{a}(2,2,1)$ by the other bijections.

Sage Example 1.16. We can demonstrate the previous example in SAGE. Using the class AfFinePermutationGroup allows to input an affine permutation in window notation:

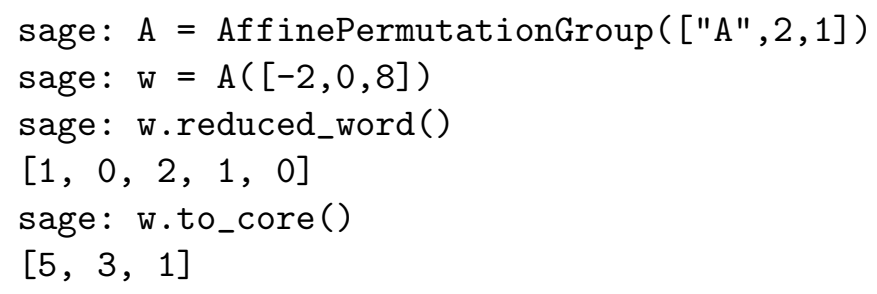

The action of $t_{i j}$ on the two line notation for $w$ can be translated into the action for $t_{i j}$ on the two line notation for $w^{-1}$. Since we have $\left(t_{i j} w\right)^{-1}=w^{-1} t_{i j}$, then the same action of left multiplication by $t_{i j}$ on $w$ has the effect of exchanging $w^{-1}(i+r n)$ and $w^{-1}(j+r n)$ in the two line notation for $w^{-1}$. Similarly, right multiplication by $t_{i j}$ on $w$ has the effect of exchanging the values of $i+r n$ and $j+r n$ in the two line notation for $w^{-1}$.

As in our example above, the two line notation for $w^{-1}$ keeps track of the outline of the $(k+1)$-core representing the affine permutation. The two line notation for $w^{-1}$ represents an infinite path where the 0 and negative values represent South steps and the positive values represent East steps. There is some point $d^{\prime}$ for which all steps before are South and another point $D^{\prime}$ where all steps after are East. Between $d^{\prime}$ and $D^{\prime}$ there is a path which traces the outline of a $(k+1)$-core. Notice that when $w=w^{-1}$ is the identity, then $d^{\prime}=0$ and $D^{\prime}=1$ and the path between these two points represents the empty core.

We can work out precisely what the action of the transpositions $s_{i}$ and $t_{i j}$ are on this path and we will see that left multiplication by $s_{i}$ on $w$ has the same effect on this path 
as the action that $s_{i}$ has on the $(k+1)$-core given in Definition 1.10. If left multiplication on $w$ by an $s_{i}$ increases the length by 1 , then on the sequence of values of $w^{-1}(i)$ this has the effect of interchanging a negative value which lies to the left of a positive value. On the path consisting of South steps for negative values and East steps for positive values, interchanging a South step that comes just before an East step has the effect of adding a cell on the path representing the core.

Although we have limited ourselves to affine permutations, $k$-bounded partitions and $k+1$-cores, we note that there are are other useful ways to describe the set such as with abaci or bit sequences that we do not discuss.

\subsection{Weak order and horizontal chains}

Because our indexing set comes from a quotient of $\tilde{S}_{k+1}$, and every Coxeter system is naturally equipped with the weak and the strong (Bruhat) orders, the close study of the weak and strong order posets on $\tilde{S}_{k+1}$ is called for. Here we examine posets that are isomorphic to the weak subposet on affine Grassmannian elements and whose vertices are given by the set of $k$-bounded partitions and by the set of $(k+1)$-cores.

The (left) weak order is defined by saying that $w$ is less than or equal to $v$ in $\tilde{S}_{k+1}$ if and only if there is some $u \in \tilde{S}_{k+1}$ such that $u w=v$ and $\ell(u)+\ell(w)=\ell(v)$. We denote a cover in (left) weak order by $\rightarrow_{k}$, that is, $w \rightarrow_{k} v$ if and only if there is some $s_{i} \in \tilde{S}_{k+1}$ such that $s_{i} w=v$ and $\ell(w)+1=\ell(v)$. The affine Weyl group of type $A$ forms a lattice under inclusion in the weak order (this is known due to results of Waugh [155]).

Now given the bijection between $\tilde{S}_{k+1} / S_{k+1}$ and $k$-bounded partitions or $(k+1)$-cores, it is natural to question how weak order $\rightarrow_{k}$ is characterized on these other sets. On the set of $(k+1)$-cores, the weak order relation can be framed in terms of the action of the elements $s_{i}$. This result can be found in $[103,112]$ and is restated in a similar form in $[81$, Lemma 8.6].

Proposition 1.17. If $\kappa$ and $\tau$ are $(k+1)$-cores with $|\kappa|_{k+1}=|\tau|_{k+1}+1$, then $\tau \rightarrow_{k} \kappa$ if and only if there exists an $i$ such that $\kappa=s_{i} \cdot \tau$.

Remark 1.18. It follows that $\rightarrow_{k}$ can also be characterized on $(k+1)$-cores by $\tau \rightarrow_{k} \kappa$ if and only if all cells in $\kappa / \tau$ have the same $k+1$-residue.

The characterization of the weak order poset on $\tilde{S}_{n} / S_{n}$ on the level of $k$-bounded partitions is inspired by viewing the Young covering relation as $\lambda \rightarrow \mu$ if $\lambda \subseteq \mu$ and $\lambda^{\prime} \subseteq \mu^{\prime}$ and $|\lambda|+1=|\mu|$. Of course, $\lambda^{\prime} \subseteq \mu^{\prime}$ if and only if $\lambda \subseteq \mu$. However, working on the subset of $k$-bounded partitions, there is a generalization of conjugation under which this is not a superfluous condition.

Definition 1.19. Let $\lambda$ be a $k$-bounded partition. Then the $k$-conjugate of $\lambda$ is defined as

$$
\lambda^{\omega_{k}}:=\mathfrak{p}\left(\mathfrak{c}(\lambda)^{\prime}\right)
$$




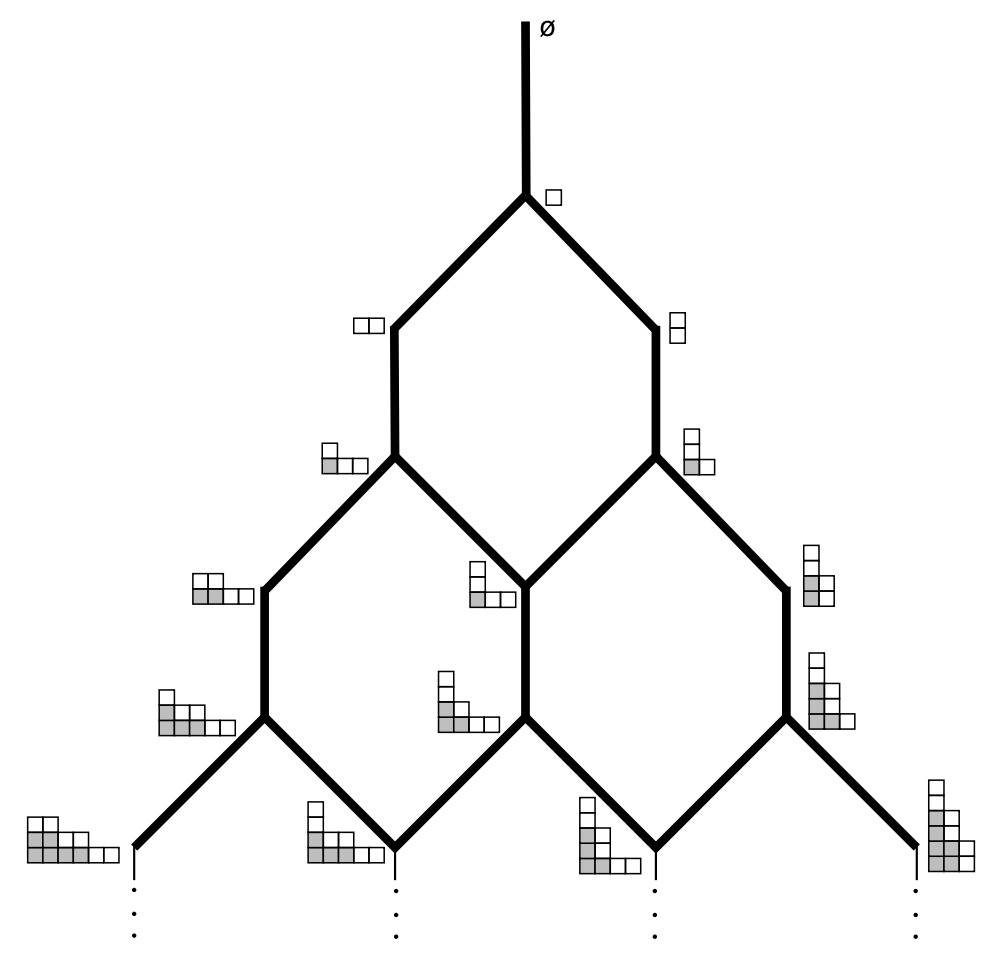

Figure 2.2: The lattice of 3-cores (up to those of length 6), which correspond to the 2-bounded partitions, ordered by the weak order.

Sage Example 1.20. We can obtain the 4-conjugate of the partition $(4,3,3,3,2,2,1)$ from the last partition in Example 1.5 by reading off the column lengths of the unshaded boxes in each column. In SAGE:

$$
\begin{aligned}
& \text { sage: } 1 \mathrm{a}=\operatorname{Partition}([4,3,3,3,2,2,1]) \\
& \text { sage: } \mathrm{la}_{\mathrm{k}} \text { conjugate }(4) \\
& {[3,2,2,2,2,1,1,1,1,1,1,1]}
\end{aligned}
$$

Proposition 1.21. ([95, Corollary 25]) For $k$-bounded partitions $\lambda$ and $\mu, \lambda \rightarrow_{k} \mu$ if and only if $\lambda \subseteq \mu, \lambda^{\omega_{k}} \subseteq \mu^{\omega_{k}}$, and $|\lambda|+1=|\mu|$.

We now turn our attention to a distinguished set of saturated chains. Recall that standard tableaux can be viewed as saturated chains in the Young lattice. This notion can be generalized to semi-standard tableaux. A semi-standard tableau is an increasing sequence of partitions in the Young lattice such that two adjacent partitions in this sequence differ by a horizontal strip. Horizontal strips are skew shapes with at most one cell in any 


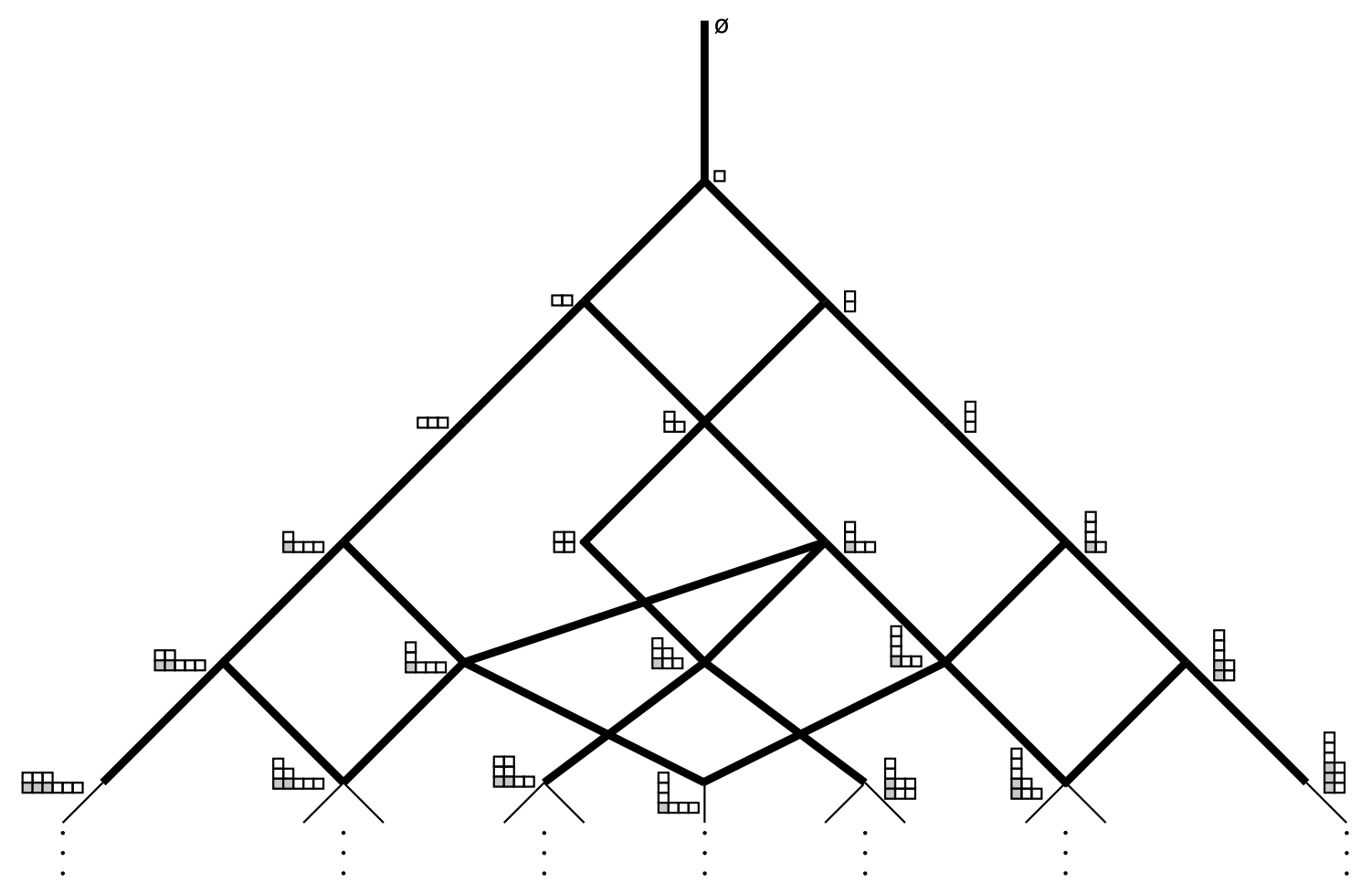

Figure 2.3: The lattice of 4-cores (up to those of length 6), which correspond to the 3-bounded partitions, ordered by the weak order.

column. As we will see in Section 2.1 horizontal strips are fundamental in the formulation of the Pieri rule for Schur functions. The analogue of horizontal strips in the affine setting was introduced in [95]. As we will see in Section 2.2 these will play a central role in the combinatorics of $k$-Schur functions.

Crudely, we define a weak horizontal strip of size $r \leq k$ to be a horizontal strip $\kappa / \tau$ of $(k+1)$-cores $\kappa$ and $\tau$ such that there exists a saturated chain

$$
\tau \rightarrow_{k} \tau^{(1)} \rightarrow_{k} \tau^{(2)} \rightarrow_{k} \cdots \rightarrow_{k} \tau^{(r)}=\kappa .
$$

It is helpful to instead think of these strips as the skew $\kappa / \tau$ of $(k+1)$-cores $\kappa$ and $\tau$, where

$$
\begin{aligned}
& \kappa / \tau \text { is a horizontal strip } \\
& |\kappa|_{k+1}=|\tau|_{k+1}+r
\end{aligned}
$$

there are exactly $r$ residues in the set of cells of $\kappa / \tau$.

We discussed how the weak order is naturally realized on $k$-bounded partitions and 
affine Grassmannian elements and it is also worthwhile to rephrase the notion of strips in these different contexts.

In the $k$-bounded partition framework (for example, useful in $[92,93,94,96,7]$ ), the notion of weak horizontal strip is defined to be a horizontal strip $\mu / \lambda$ where $\mu^{\omega_{k}} / \lambda^{\omega_{k}}$ is a vertical strip. This characterization is motivated by the following result about weak horizontal strips.

Proposition 1.22. ([95, Section 9] Let $\tau \subseteq \kappa$ be $(k+1)$-cores. Then $\kappa / \tau$ forms a weak horizontal strip if and only if $\mathfrak{p}(\kappa) / \mathfrak{p}(\tau)$ is a horizontal strip and $\mathfrak{p}\left(\kappa^{\prime}\right) / \mathfrak{p}\left(\tau^{\prime}\right)$ is a vertical strip.

It is important to note that it is not sufficient to characterize $\kappa / \tau$ being a weak horizontal strip by assuming that $\mathfrak{p}(\kappa) / \mathfrak{p}(\tau)$ is a horizontal strip. A good example of this can be observed in Figure 2.3. Consider the 4-cores $\kappa=(4,1)$ and $\tau=(2,1)$. We note that $\mathfrak{p}(\kappa)=(3,1)$ and $\mathfrak{p}(\tau)=(2,1)$ and so even though we see that $\mathfrak{p}(\kappa) / \mathfrak{p}(\tau)$ is a horizontal strip, there does not exist a path from the 4-core $(2,1)$ to $(4,1)$ in the lattice. If we consider the conjugate partitions, we see that $\mathfrak{p}\left(\kappa^{\prime}\right)=(1,1,1,1)$ and $\mathfrak{p}\left(\tau^{\prime}\right)=(2,1)$ and $\operatorname{so} \mathfrak{p}\left(\kappa^{\prime}\right) / \mathfrak{p}\left(\tau^{\prime}\right)$ is not a vertical strip.

Before we discuss the notion of weak horizontal strip in the framework of affine permutations, we give an alternative description of the $k$-conjugate and the map $\mathfrak{c}$ from $k$-bounded partitions to $(k+1)$-cores that was communicated to us by Karola Mészáros [122]. Let $\lambda=\left(\lambda_{1}, \lambda_{2}, \ldots, \lambda_{\ell}\right)$ be a $k$-bounded partition. Start from the longest part of $\lambda$, namely $\lambda_{1}$, and successively connect a row of length $i$ to the $(k+1-i)$ th row above it (in other words, skip $k-i$ rows). Call this a string. Repeat this with the next longest part that is not yet part of a string until all parts of $\lambda$ are part of a string. Denote by $\left\{\ell_{1}^{(j)}, \ell_{2}^{(j)}, \ldots\right\}$ the parts of $\lambda$ in the $j$ th string where $1 \leq j \leq r$. Then

$$
\left(\lambda^{\omega_{k}}\right)^{\prime}=\left(\ell_{1}^{(1)}+\ell_{2}^{(1)}+\cdots, \ell_{1}^{(2)}+\ell_{2}^{(2)}+\cdots, \ldots, \ell_{1}^{(r)}+\ell_{2}^{(r)}+\cdots\right) .
$$

Similarly, the $i$ th part of $\mathfrak{c}(\lambda)$ is obtained by adding all elements in the string containing $\lambda_{i}$ that are smaller or equal to $\lambda_{i}$ (or equivalently, all elements in the string of $\lambda_{i}$ above $\lambda_{i}$ ).

Example 1.23. Let $\lambda=(3,3,3,2,1)$ with $k=4$. Then the strings are as follows:

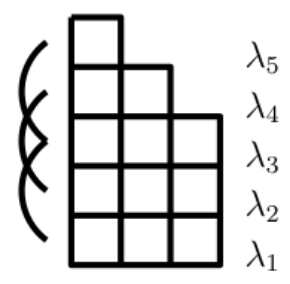


and

$$
\begin{aligned}
\left(\lambda^{\omega_{4}}\right)^{\prime} & =\left(\lambda_{1}+\lambda_{3}+\lambda_{5}, \lambda_{2}+\lambda_{4}\right)=(7,5), \\
\mathfrak{c}(\lambda) & =\left(\lambda_{1}+\lambda_{3}+\lambda_{5}, \lambda_{2}+\lambda_{4}, \lambda_{3}+\lambda_{5}, \lambda_{4}, \lambda_{5}\right)=(7,5,4,2,1) .
\end{aligned}
$$

Lastly, we interpret horizontal chains in the framework of affine permutations. In particular, weak horizontal strips are the cyclically decreasing elements of $\tilde{S}_{k+1} / S_{k+1}$. These are affine Grassmannian elements $w$ where $w=s_{i_{1}} \cdots s_{i_{\ell}}$ for a sequence $i_{1} \cdots i_{\ell}$ such that no number is repeated and $j$ precedes $j-1$ (taken modulo $k+1$ ) when both $j, j-1 \in\left\{i_{1}, \ldots, i_{\ell}\right\}$. Then, based on the following proposition, a weak horizontal strip can be thought of as a pair of affine Grassmannian elements $v$ and $w$ where $u w=v$ and $\ell(u)+\ell(w)=\ell(v)$ for some cyclically decreasing $u$.

Proposition 1.24. Let $\tau \subseteq \kappa$ be $(k+1)$-cores. Then $\kappa / \tau$ forms a weak horizontal strip if and only if $\kappa=s_{i_{1}} \cdots s_{i_{\ell}} \tau$ for some cyclically decreasing element $w=s_{i_{1}} \cdots s_{i_{\ell}}$.

\subsection{Cores and the strong order of the affine symmetric group}

We are now ready to discuss the strong (Bruhat) order, starting from the core viewpoint $[123,103]$. A strong cover is defined on $k+1$ cores by

$$
\tau \Rightarrow_{k} \kappa \Longleftrightarrow|\mathfrak{p}(\tau)|+1=|\mathfrak{p}(\kappa)| \text { and } \quad \tau \subseteq \kappa .
$$

When a pair of cores satisfies $\tau \Rightarrow_{i} \kappa$, their skew diagram has is made up of ribbons. To be precise, the head of a connected ribbon is the southeast most cell of the ribbon and the tail is the northwest most corner. Then (see [81, Proposition 9.5]):

- each connected component of $\kappa / \tau$ is a ribbon and they are all identical translates each other;

- the residues of the heads of the connected components must all be the same and must lie on consecutive positions of those residues (the term 'consecutive' here means that if two heads are separated by a multiple of $k+1$ cells by a taxicab distance then there is one which is exactly $k+1$ distance that is in-between).

A strong marked cover is a strong cover along with a value $c$ which indicates the content of the head of one of the copies of the ribbons. More precisely, we define a marking as a triple $(\kappa, \tau, c)$ where $\kappa, \tau$ are $(k+1)$-cores such that $\tau \Rightarrow_{k} \kappa$ and $c$ is a number which is $j-i$ for the cell (the diagonal index of the cell) at position $(i, j)$ of the south-east most cell of the connected component of $\kappa / \tau$ which is marked.

Example 1.25. Consider the 4-cores

$$
\tau=(19,16,13,10,7,7,5,5,3,3,1,1,1) \Rightarrow_{3}(22,19,16,13,10,7,5,5,3,3,1,1,1)=\kappa
$$


which correspond to the skew diagram

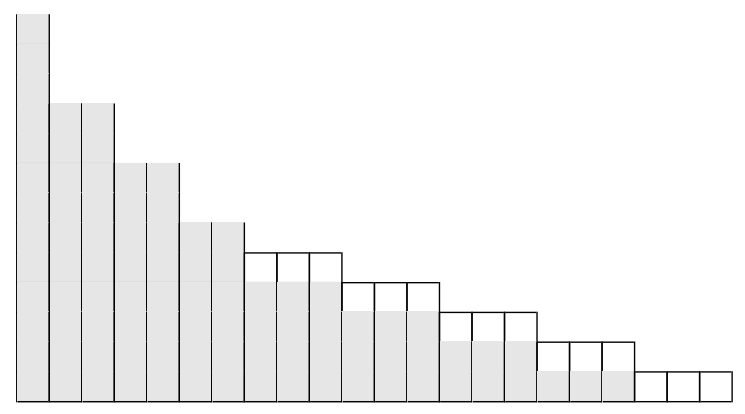

This is a relatively large example, where $\kappa / \tau$ contains 5 different copies of 3 cells in a row. The marking, $c$, can be any one of the 5 values representing the content of the rightmost cell in the connected component, $c \in\{21,17,13,9,5\}$.

Example 1.26. The diagram for the poset of the strong order at $k=2$ is given in Figure 2.4 and it can be read off the diagram that the strong covers of $(3,1,1)$ with respect to this order are $(5,3,1),(4,2,1,1)$ and $(3,2,2,1,1)$ because $(3,1,1)$ is contained in each of these 3 -cores. Note that it is not true that there is containment of the corresponding 2-bounded partitions since $\mathfrak{p}(3,1,1)=(2,1,1)$ and $\mathfrak{p}(3,2,2,1,1)=(1,1,1,1,1)$.

In particular, if $\tau=(3,1,1)$ and $\kappa=(5,3,1)$, then $(3,1,1) \Rightarrow_{2}(5,3,1)$ because $(5,3,1) /(3,1,1)$ consists of two copies of a connected horizontal strip. This means that $((5,3,1) /(3,1,1), 1)$ and $((5,3,1) /(3,1,1), 4)$ are strong marked covers since the cells $(2,3)$ and $(1,5)$ are the coordinates of the heads of the horizontal strips and they have content 1 and 4 respectively. These are represented by the skew tableaux

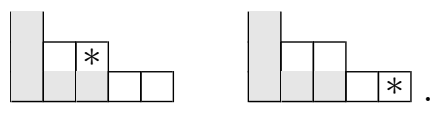

Although we started with a discussion of strong order in the setting of $k+1$-cores, it comes from ordering elements $u, w$ of the Coxeter system $\tilde{S}_{k+1}$ by

$$
w \Rightarrow_{k} u \Longleftrightarrow t_{i j} w=u \text { and } \ell(w)+1=\ell(u) .
$$

The notion of marked covers can be interpreted in this framework as well.

Proposition 1.27. [81, Section 2.3] Let $\tau, \kappa$ be two $(k+1)$-cores such that $\tau \Rightarrow_{k} \kappa$ and assume there is a marking of $\kappa / \tau$ at diagonal $j-1$ and $i$ is the diagonal index of the tail of the marked ribbon. Let $w$ be the affine Grassmannian permutation corresponding to $\tau$ and $u$ the affine Grassmannian permutation corresponding to $\kappa$. Then we have

$$
\text { - } w^{-1}(i) \leq 0<w^{-1}(j) \text {. }
$$




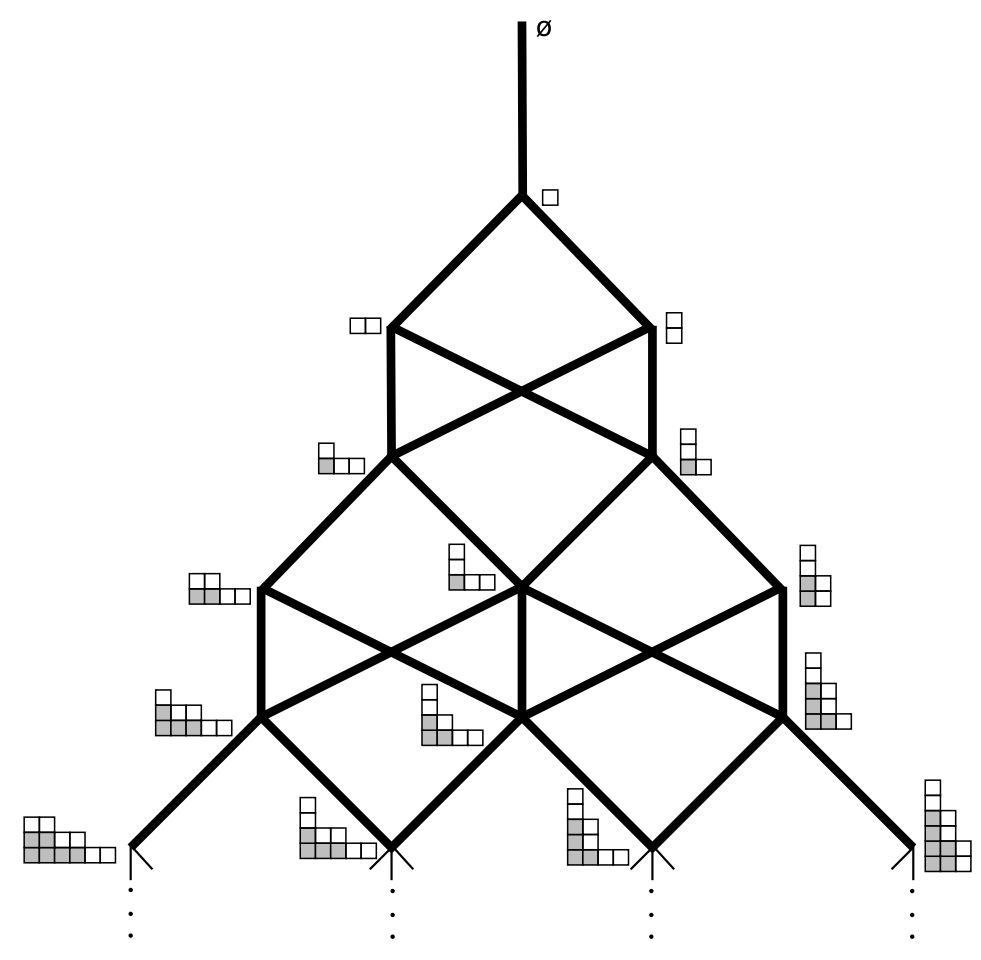

Figure 2.4: The poset of 3-cores (up to those of length 6), ordered by the strong order (no markings)

- $t_{i j} w=u$.

- The number of connected ribbons which are below the marked one is $\left(-w^{-1}(i)-a\right) / n$ where $a=-w^{-1}(i)(\bmod n)$ (the representative between 0 and $\left.n\right)$.

- The number of connected ribbons which are above the marked one is $\left(w^{-1}(j)-b\right) / n$ where $b=w^{-1}(j)(\bmod n)$ and the total number of connected components in $\kappa / \tau$ is $1+\left(-w^{-1}(i)+w^{-1}(j)-a-b\right) / n$.

- The number of cells in the ribbon is $j-i$.

- The height of the ribbon is the number of $d$ such that $i \leq d<j$ such that $w^{-1}(d) \leq 0$.

Example 1.28. Consider the 3-core $\tau=(5,3,1)$ which corresponds to the reduced word $w=s_{1} s_{0} s_{2} s_{1} s_{0}$ and which is also given by its action on the integers by $[w(1), w(2), w(3)]=$ 


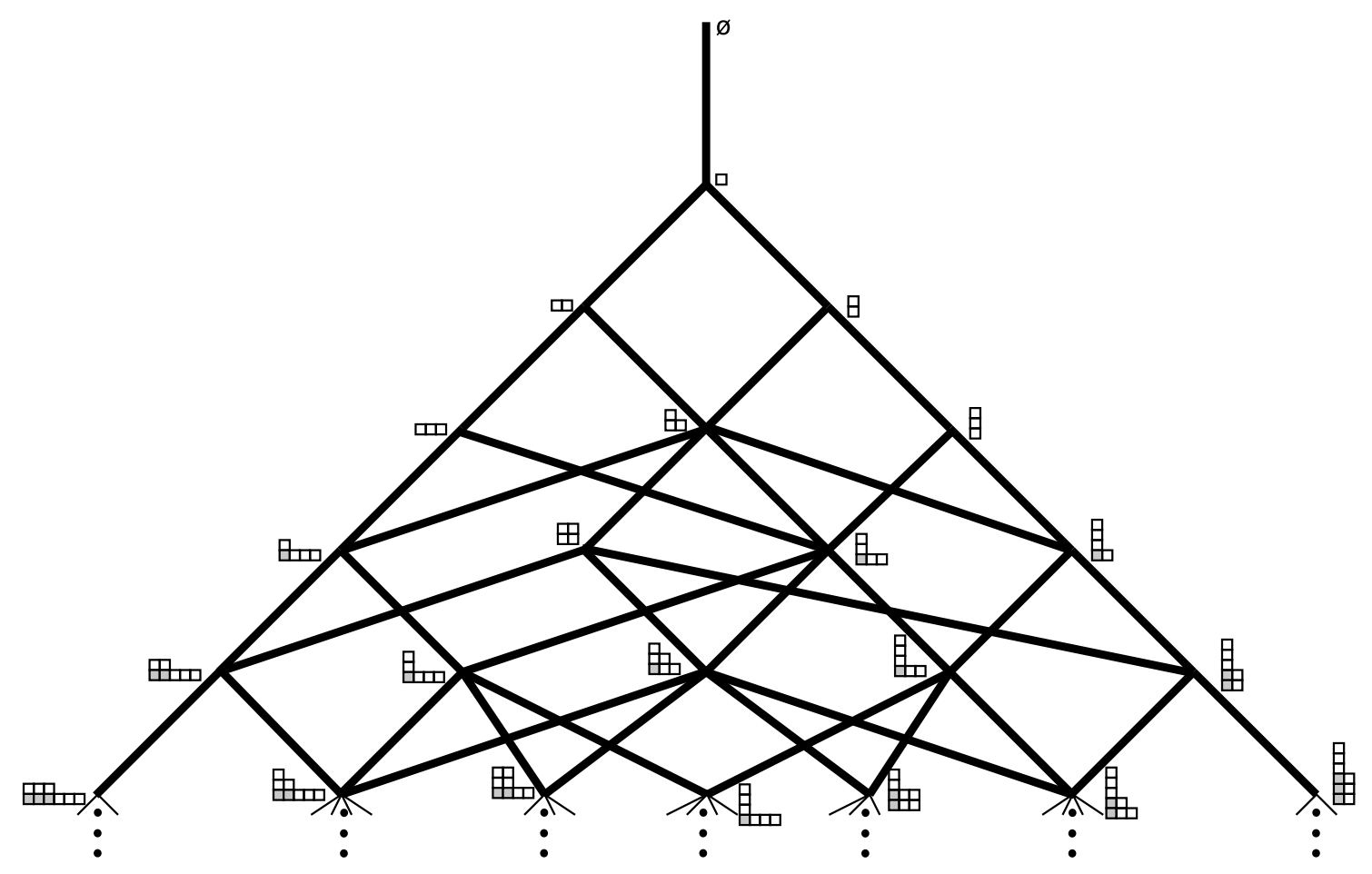

Figure 2.5: The poset of 4-cores (up to those of length 6), ordered by the strong order (no markings)

$[-2,0,8]$. Then $t_{-10}=t_{23}=t_{56}=s_{2}$ and $\kappa=t_{-10} \cdot(5,3,1)=(6,4,2)$.

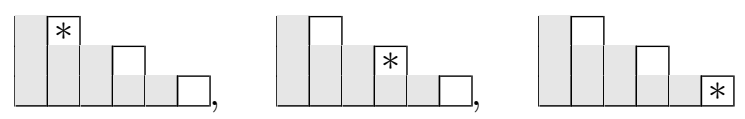

The three markings are on diagonal -1 and 2 and 5 . Moreover since $w^{-1}(-1)=-6 \leq$ $0<w^{-1}(0)=2, w^{-1}(2)=-3 \leq 0<w^{-1}(3)=5$ and $w^{-1}(5)=0 \leq 0<w^{-1}(6)=8$, these three transpositions satisfy the conditions of the proposition for each of the three marked strong covers. The right action is expressed as the element $w t_{-62}=w t_{-35}=w t_{08}$.

The number of connected components is equal to $1+\left(-w^{-1}(i)+w^{-1}(j)-a-b\right) / n$ which for $j=0$ and $i=-1$ amounts to $1+(6+2-0-2) / 3=3$.

Example 1.29. The 4-core $\tau$ in Example 1.25 corresponds to the reduced word

$$
w=s_{1} s_{2} s_{0} s_{3} s_{1} s_{2} s_{0} s_{3} s_{1} s_{2} s_{1} s_{0} s_{3} s_{1} s_{2} s_{1} s_{0} s_{3} s_{0} s_{1} s_{2} s_{1} s_{0} s_{3} s_{2} s_{1} s_{0}
$$


and $t_{19,22} w=t_{15,18} w=t_{11,14} w=t_{7,10} w=t_{3,6} w$ represent the five strong covers with the left action and $w t_{0,19}=w t_{-4,15}=w t_{-8,11}=w t_{-12,7}=w t_{-16,3}$ represent the five strong covers with the right action.

Remark 1.30. Recall that for two elements $w, w^{\prime} \in S_{n}$ with $\ell\left(w^{\prime}\right)=\ell(w)+1, w^{\prime}$ is a cover of $w$ in the (left) weak order if

$$
s_{i} w=w^{\prime}
$$

for some simple transposition $s_{i}$ and $w^{\prime}$ is a cover of $w$ in the strong (or Bruhat) order if

$$
t_{i j} w=w^{\prime}
$$

for some transposition $t_{i j}$. By analogy, since $\Rightarrow_{k}$ is a left multiplication by an affine transposition (Proposition 1.27) and $\rightarrow_{k}$ is left multiplication by a simple affine transposition on cores (from Definition 1.10), the cover relations are called strong and weak covers, respectively.

We have included the Hasse diagrams for the weak and strong orders for the poset of 3 and 4-cores up to those of length 6 in Figures 2.2 through 2.5. Note that the strong order on cores does not form a lattice.

Sage Example 1.31. We can produce the weak and strong covers of a given core

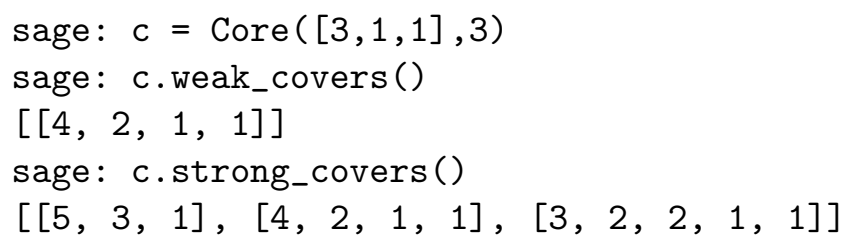

as well as compare two $(k+1)$-cores with respect to weak and strong order

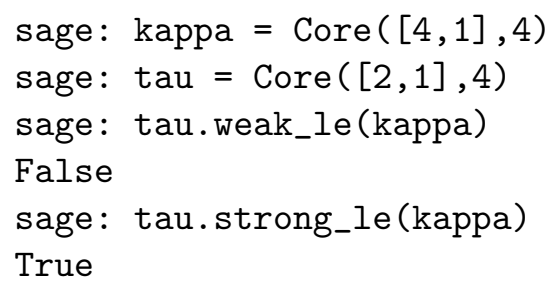

Figure 2.3 can be reproduced in SAgE via:

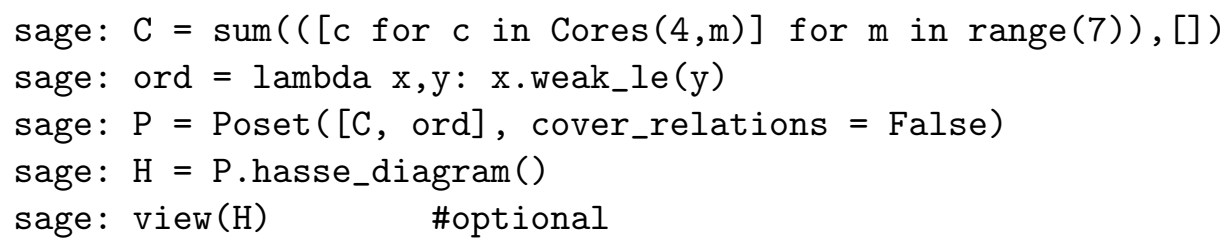


As with the weak order poset, we are also concerned with certain chains in the strong order poset. A strong marked horizontal strip of size $r$ is a succession of strong marked covers,

$$
\kappa^{(0)} \Rightarrow_{k} \kappa^{(1)} \Rightarrow_{k} \kappa^{(2)} \Rightarrow_{k} \cdots \Rightarrow_{k} \kappa^{(r)},
$$

where the markings $c_{i}$ associated to $\kappa^{(i-1)} \Rightarrow_{k} \kappa^{(i)}$ satisfy $c_{1}<c_{2}<\cdots<c_{r}$.

Example 1.32. Consider the following sequence of 4-cores with the markings indicated on the diagram.

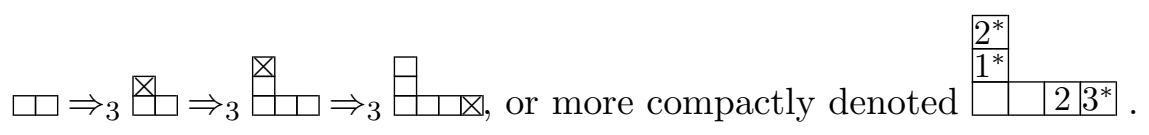

This is not a strong marked horizontal strip because the markings $c_{1}=-1, c_{2}=-2, c_{3}=3$ are not increasing. However, the same set of 4-cores with markings

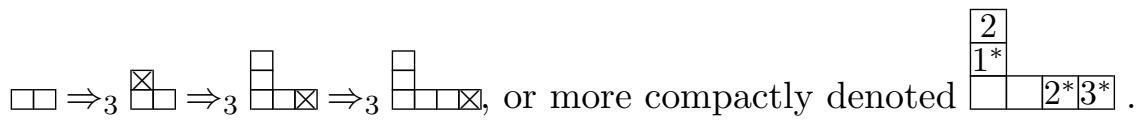

as shown have $c_{1}=-1<c_{2}=2<c_{3}=3$ and hence this is a strong marked horizontal strip.

Remark 1.33. It is worth pointing out that when $k$ is large, a succession of strong marked covers,

$$
\kappa^{(0)} \Rightarrow_{k} \kappa^{(1)} \Rightarrow_{k} \kappa^{(2)} \Rightarrow_{k} \cdots \Rightarrow_{k} \kappa^{(r)},
$$

reduces to a saturated chain in Young's lattice where the condition that $c_{1}<c_{2}<\cdots<c_{r}$ implies that $\kappa^{(r)} / \kappa^{(0)}$ is a horizontal strip.

\subsection{Symmetric functions}

The ring of symmetric functions shall be defined as

$$
\Lambda=\mathbb{Q}\left[h_{1}, h_{2}, h_{3}, \ldots\right],
$$

the ring of polynomials in the generators $h_{r}$. Here we are considering the ring $\Lambda$ without reference to 'variables' for which there is a symmetric group action but we will now make explicit the connection with symmetric polynomials and symmetric series.

Let $\sigma$ be a permutation that acts on the variables $\left\{x_{1}, x_{2}, x_{3}, \ldots, x_{m}\right\}$ by $\sigma\left(x_{i}\right)=x_{\sigma_{i}}$ and this action extends to polynomials. We call a polynomial $S_{m}$-invariant (or symmetric) if $\sigma f\left(x_{1}, x_{2}, \ldots, x_{m}\right)=f\left(x_{1}, x_{2}, \ldots, x_{m}\right)$ for all $\sigma \in S_{m}$. The ring $\Lambda$ is identified with functions which are symmetric series in an infinite set of variables by setting

$$
h_{r}[X]=\sum_{1 \leq i_{1} \leq i_{2} \leq \cdots \leq i_{r}} x_{i_{1}} x_{i_{2}} \cdots x_{i_{r}}
$$


and symmetric polynomials are then just a specialization of these series with a finite number of variables, $h_{r}\left[X_{m}\right]=\sum_{1 \leq i_{1} \leq i_{2} \leq \cdots \leq i_{r} \leq m} x_{i_{1}} x_{i_{2}} \cdots x_{i_{r}}$.

For each partition $\lambda=\left(\lambda_{1}, \lambda_{2}, \ldots, \lambda_{\ell(\lambda)}\right)$, we set

$$
h_{\lambda}[X]:=h_{\lambda_{1}}[X] h_{\lambda_{2}}[X] \cdots h_{\lambda_{\ell(\lambda)}}[X] .
$$

The set of these symmetric series forms a linear basis for an algebra isomorphic to $\Lambda$. We will consider various bases for $\Lambda$. One such basis is the monomial basis

$$
m_{\lambda}[X]=\sum_{\operatorname{sort}(\alpha)=\lambda} x_{1}^{\alpha_{1}} x_{2}^{\alpha_{2}} \cdots x_{\ell(\alpha)}^{\alpha_{\ell(\alpha)}}
$$

where the sum is over all sequences $\alpha$ such that if the parts are arranged in weakly decreasing order the resulting sequence is the partition $\lambda$. It is not hard to see from the definitions that the generators of $\Lambda$ are related to the monomial symmetric functions by

$$
h_{r}[X]=\sum_{\lambda \vdash r} m_{\lambda}[X] .
$$

The $h_{r}$ are known as the complete homogeneous generators and the their products will be referred to as the complete homogeneous or simply homogeneous basis.

The power sum generators are the elements

$$
p_{r}[X]=m_{(r)}[X]=\sum_{i \geq 1} x_{i}^{r}
$$

and the elementary generators are defined as

$$
e_{r}[X]=m_{\left(1^{r}\right)}[X]=\sum_{1 \leq i_{1}<i_{2}<\cdots<i_{r}} x_{i_{1}} x_{i_{2}} \cdots x_{i_{r}} .
$$

The monomials in these sets of generators, $p_{\lambda}[X]:=p_{\lambda_{1}}[X] p_{\lambda_{2}}[X] \cdots p_{\lambda_{\ell(\lambda)}}[X]$ and $e_{\lambda}[X]:=e_{\lambda_{1}}[X] e_{\lambda_{2}}[X] \cdots e_{\lambda_{\ell(\lambda)}}[X]$ also form bases for the space $\Lambda$ indexed by partitions.

The variable $X$ in these symmetric functions is, for the moment, superfluous notation and to make certain formulas more compact we will drop $[X]$ when it is implicit that it is there. However, we will later consider transformations on the ring of symmetric functions by adding notation to $[X]$ and there will be times that the $[X]$ will be used to indicate that the expression it is attached to is a symmetric function.

One place where it will be necessary to keep the reference to the variables explicit is in the use of a few 'plethystic' expressions involving parameters $q$ and $t$. We may extend the notation defined above to include all rational expressions in variables $q, t, x_{1}, x_{2}, x_{3}, \ldots$, $E=E\left(x_{1}, x_{2}, \ldots ; q, t\right)$, then

$$
p_{r}[E]=E\left(x_{1}^{r}, x_{2}^{r}, \ldots ; q^{r}, t^{r}\right) .
$$


Note that by using the expression $E$ as the infinite sum $X=x_{1}+x_{2}+x_{3}+\cdots$, the notation in Equation (1.19) is consistent with the expression in Equation (1.17).

In particular we will frequently use the notation $f\left[\frac{X}{1-t}\right], f[X(1-t)]$ and $f\left[X \frac{1-q}{1-t}\right]$ to represent the symmetric function $f[X]$ with $p_{r}[X]$ replaced with $p_{r}[X] /\left(1-t^{r}\right), p_{r}[X]\left(1-t^{r}\right)$ and $p_{r}[X] \frac{1-q^{r}}{1-t^{r}}$ respectively. This transformation is sometimes also denoted by

$$
\theta_{q t} f[X]=f\left[X \frac{1-q}{1-t}\right]
$$

When we need to use a finite number of variables, the expression $X_{m}=x_{1}+x_{2}+$ $x_{3}+\cdots+x_{m}$ is used to indicate that $p_{r}\left[X_{m}\right]=\sum_{i=1}^{m} x_{i}^{r}$. Normally we consider symmetric functions in an arbitrary alphabet which can be specialized appropriately and we assume that there is an implicit $[X]$ following all symmetric function expressions where no variables are specified.

The three types of generators are related by

$$
\begin{array}{lr}
\sum_{i=0}^{r}(-1)^{r-i} h_{i} e_{r-i}=0 & r h_{r}=\sum_{i=1}^{r} h_{r-i} p_{i} \\
\sum_{i=0}^{r}(-1)^{r-i} i h_{i} e_{r-i}=p_{r} & r e_{r}=\sum_{i=1}^{r}(-1)^{i-1} e_{r-i} p_{i} .
\end{array}
$$

These relations are sufficient to express any one of the generators $\left\{e_{r}, h_{r}, p_{r}\right\}$ in terms of another.

There is a scalar product on the ring of symmetric functions for which the monomial and homogeneous symmetric functions are orthonormal. We have also,

$$
\left\langle h_{\lambda}, m_{\mu}\right\rangle=\left\langle p_{\lambda}, p_{\mu} / z_{\mu}\right\rangle=\delta_{\lambda \mu}:= \begin{cases}1 & \text { if } \lambda=\mu \\ 0 & \text { otherwise }\end{cases}
$$

where $z_{\mu}$ is defined in Equation (1.1).

One powerful use of this scalar product is that it allows us to compute a single coefficient in the expansion of a symmetric function in terms of these bases. If $f \in \Lambda$, then $\left\langle f, h_{\mu}\right\rangle$ is the coefficient of $m_{\mu}$ in $f$. That is, if $f=\sum_{\gamma} c_{\gamma} m_{\gamma}$, then

$$
\left\langle f, h_{\mu}\right\rangle=\sum_{\gamma} c_{\gamma}\left\langle m_{\gamma}, h_{\mu}\right\rangle=c_{\mu} .
$$

Similarly $\left\langle f, m_{\mu}\right\rangle$ is the coefficient of $h_{\mu}$ in $f$ and $\left\langle f, p_{\mu} / z_{\mu}\right\rangle$ is equal to the coefficient of $p_{\mu}$ in $f$.

Sage Example 1.34. We now show how to create various bases in SAGE and how to obtain the coefficients of a given symmetric function using the computer. We begin by defining the homogeneous and monomial bases: 


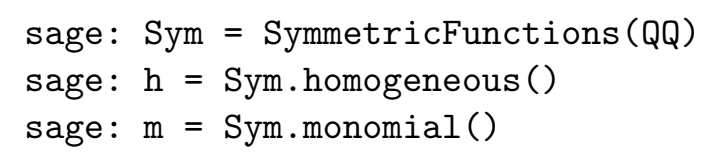

Then we define a symmetric function $f$ and expand it in terms of the monomial basis:

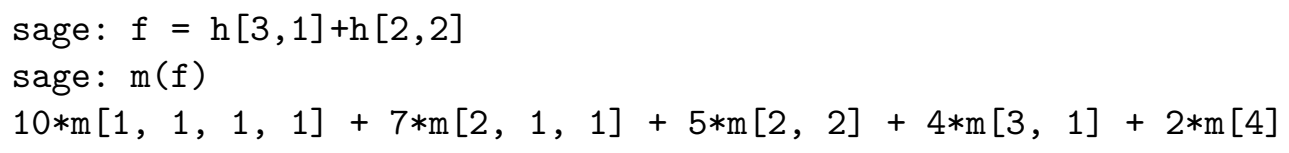

There are several ways to obtain the coefficients of a given term. Both of the following yield the coefficient of $m_{211}$ in $f$ :

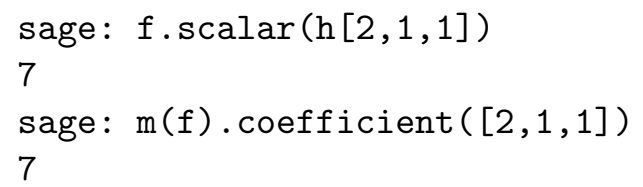

The order in which bases are multiplied and added determines which is the output basis. For instance to demonstrate (1.22) we consider the following two equivalent expressions:

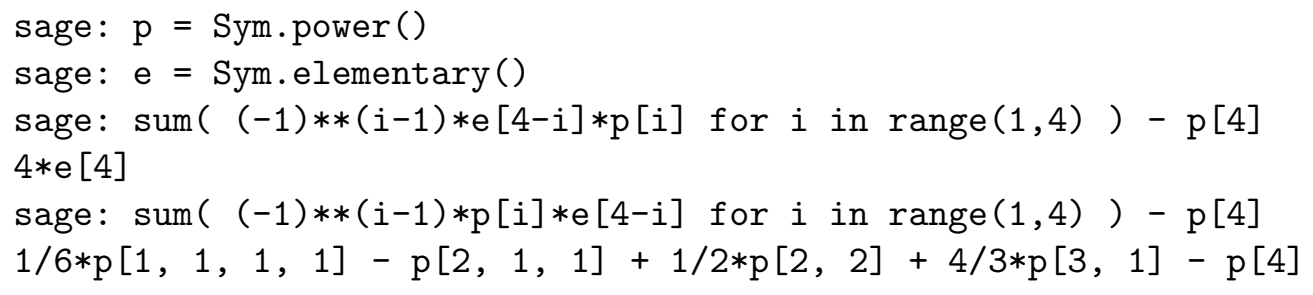

\subsection{Schur functions}

A combinatorial definition of the Schur functions is given in Section 2 which we shall generalize to the $k$-Schur functions in Section 2.2 and the dual $k$-Schur functions in Section 2.4. For now we start with two (equivalent) algebraic definitions.

Definition 1.35. The Schur functions $s_{\lambda}$ are the unique basis of $\Lambda$ for which

1. $\left\langle s_{\lambda}, s_{\mu}\right\rangle=\delta_{\lambda \mu}$ for any partitions $\lambda, \mu$;

2. $s_{\lambda}=m_{\lambda}+$ terms of the form $r_{\lambda \mu} m_{\mu}$ for partitions $\mu$ of $|\lambda|$ with $\mu<\lambda$ in dominance order.

We have chosen this as the definition of the Schur functions because it naturally generalizes to the Hall-Littlewood and Macdonald symmetric functions (which we shall introduce in the next few pages). There are many formulas known for the Schur functions which can either be taken as a defining relation or as a consequence. In the next section we shall shift perspectives and consider the Schur functions as the family of symmetric functions which satisfy the Pieri rule (see Equation (2.2)). 
Example 1.36. In the following we abbreviate $s_{(2,1)}$ by $s_{21}$ if there is no confusion about the parts. By definition we have that

$$
s_{111}=m_{111}
$$

and we may proceed by calculating Gram-Schmidt orthonormalization. For instance, since $s_{111}=m_{111}=e_{3}=h_{111}-2 h_{21}+h_{3}$ we have

$$
s_{21}=m_{21}-\left\langle m_{21}, s_{111}\right\rangle s_{111}=m_{21}+2 m_{111} .
$$

If then $s_{21}$ is expanded in the homogeneous basis, we see that it is $s_{21}=h_{21}-h_{3}$. Finally, to calculate $s_{3}$ we note that

$$
s_{3}=m_{3}-\left\langle m_{3}, s_{21}\right\rangle s_{21}-\left\langle m_{3}, s_{111}\right\rangle s_{111}=m_{3}+m_{21}+m_{111} .
$$

Sage Example 1.37. If we wanted to check Example 1.36 using SAGE, we could define $h$ and $m$ as in Sage Example 1.34 and then run

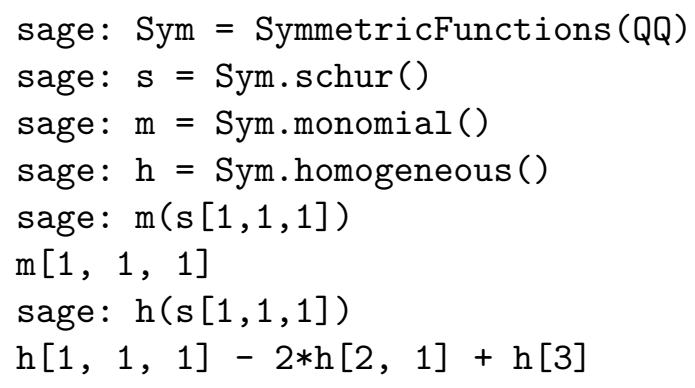

etc.. We can also obtain the expansion of the Schur functions into power sum symmetric functions:

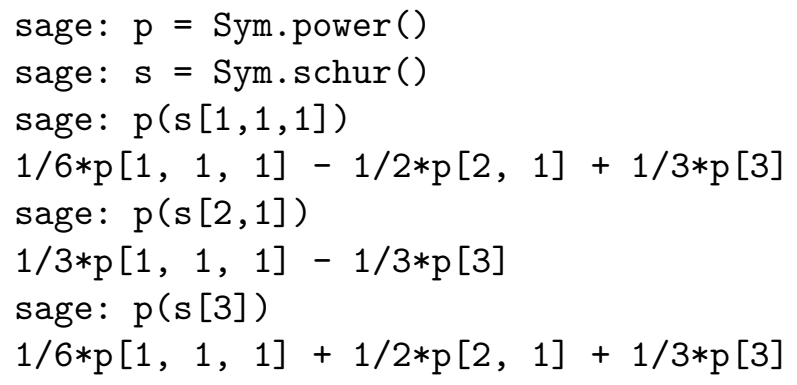

and the following calculation shows that the Schur functions are orthogonal:

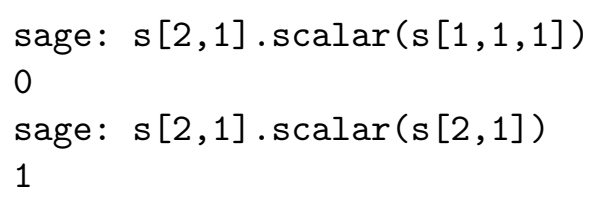


Since the Schur functions form a basis of the ring of symmetric functions $\Lambda$, a product of two Schur functions can again be expanded in terms of Schur functions:

$$
s_{\lambda} s_{\mu}=\sum_{\nu} c_{\lambda \mu}^{\nu} s_{\nu}
$$

It turns out that the coefficients $c_{\lambda \mu}^{\nu}$, called Littlewood-Richardson coefficients, are nonnegative integer coefficients. The famous Littlewood-Richardson rule [118] provides a combinatorial expression for the coefficients $c_{\lambda \mu}^{\nu}$. It says that $c_{\lambda \mu}^{\nu}$ is equal to the number of semi-standard tableaux of skew shape $\nu / \lambda$ and weight $\mu$ whose column reading word is Yamanouchi. Here $\lambda, \mu, \nu$ are partitions and a semi-standard tableau of shape $\nu / \lambda$ is a filling of the skew shape which is weakly increasing across rows and strictly increasing up columns. The weight of a tableau or word is $\mu=\left(\mu_{1}, \mu_{2}, \ldots\right)$, where $\mu_{i}$ counts the number of $i$ in the tableau or word. Furthermore, a word is Yamanouchi if all right subwords have partition weight.

Remarkably, Schur functions and their Littlewood-Richardson coefficients tie into the study of the geometry of the Grassmannian $\mathrm{Gr}_{\ell n}$ (the manifold of $\ell$-dimensional subspaces of $\left.\mathbb{C}^{n}\right)$. The cohomology ring of $\mathrm{Gr}_{\ell n}$ has a basis of Schubert classes $\sigma_{\lambda}$, indexed by shapes $\lambda \in \mathcal{P}^{\ell n}$ contained in an $\ell \times(n-\ell)$ rectangle. The intersection numbers are encoded by the structure constants of $H^{*}\left(\mathrm{Gr}_{\ell n}\right)$ in the Schubert basis:

$$
\sigma_{\lambda} \cup \sigma_{\mu}=\sum_{\nu \in \mathcal{P}^{\ell n}} c_{\lambda \mu}^{\nu} \sigma_{\nu}
$$

The explicit understanding of $H^{*}\left(\mathrm{Gr}_{\ell n}\right)$ and of these intersections is gained by Schur functions. Letting $I=\left\langle e_{n-\ell+1}, \ldots, e_{n}\right\rangle$ and $\Lambda_{(\ell)}=\mathbb{Q}\left[h_{1}, h_{2}, h_{3}, \ldots, h_{\ell}\right]$, there is an isomorphism,

$$
H^{*}\left(\mathrm{Gr}_{\ell n}\right) \cong \Lambda_{(\ell)} / I
$$

under which $\sigma_{\lambda}$ corresponds to $s_{\lambda}$. Importantly, $s_{\nu} \in I$ when $\nu \notin \mathcal{P}^{\ell n}$, and thus the structure constants of $H^{*}\left(\mathrm{Gr}_{\ell n}\right)$ are none other than the Littlewood-Richardson coefficients (1.25) for Schur function products.

The ring of symmetric functions is also endowed with a Hopf algebra structure. A systematic study of $\Lambda$ from the Hopf algebra perspective is given in [160], see also [120]. In particular, the coproduct on the Schur basis is given in terms of the Littlewood-Richardson coefficients

$$
\Delta\left(s_{\nu}\right)=\sum_{\lambda, \mu} c_{\lambda \mu}^{\nu} s_{\lambda} \otimes s_{\mu}
$$

There is also an algebraic involution on $\Lambda$ for which $\omega\left(h_{\lambda}\right)=e_{\lambda}$ and $\omega\left(p_{\lambda}\right)=(-1)^{|\lambda|-\ell(\lambda)} p_{\lambda}$ and $\omega\left(s_{\lambda}\right)=s_{\lambda^{\prime}}$.

With the scalar product it is natural to introduce the operation which is dual to multiplication. That is, for a homogeneous symmetric function $f$ of degree $k$, multiplication 
by $f$ is an operation which will raise the degree of a symmetric function by $k$, and the notation $f^{\perp}$ will represent an operator that will lower a symmetric function by degree $k$ and its action is defined as

$$
f^{\perp}(g)=\sum_{\lambda}\left\langle g, f s_{\lambda}\right\rangle s_{\lambda}=\sum_{\lambda}\left\langle g, f h_{\lambda}\right\rangle m_{\lambda}
$$

The reason why we introduce these operators is that we find that we can define 'creation operators' for the Schur functions which allow us to show that the Schur functions satisfy

the Pieri rule. Set $\mathbf{S}_{m}=\sum_{r \geq 0}(-1)^{r} h_{m+r} e_{r}^{\perp}$. The sum is apparently infinite but we need only calculate up to $r$ equal to the degree of the symmetric function it is acting on. These operators have the property that for a partition $\lambda=\left(\lambda_{1}, \lambda_{2}, \ldots, \lambda_{\ell}\right)$ and $m \geq \lambda_{1}$,

$$
\mathbf{S}_{m} s_{\lambda}=s_{\left(m, \lambda_{1}, \lambda_{2}, \ldots, \lambda_{\ell}\right)}
$$

One reason these operators are particularly useful is that commutation rules such as $p_{r} \mathbf{S}_{m}=\mathbf{S}_{m} p_{r}+\mathbf{S}_{m+r}$ and $e_{r} \mathbf{S}_{m}=\mathbf{S}_{m+1} e_{r-1}+\mathbf{S}_{m} e_{r}$ and $h_{r} \mathbf{S}_{m}=\sum_{i=0}^{r} \mathbf{S}_{m+i} h_{r-i}$ can be used to show the Murnaghan-Nakayama rule and the Pieri rules (respectively). We will also use the operators $\mathbf{S}_{m}$ to express creation operators for the Hall-Littlewood symmetric functions and a means for computing them. In particular,

$$
s_{\lambda}=\mathbf{S}_{\lambda_{1}} \mathbf{S}_{\lambda_{2}} \cdots \mathbf{S}_{\lambda_{\ell(\lambda)}}(1) .
$$

\subsection{Hall-Littlewood symmetric functions}

The Hall-Littlewood symmetric functions have a definition which is similar to that of the Schur functions. These functions form a basis of the ring of symmetric functions over a field containing a parameter $t$. We work in the fraction field over the polynomials in the parameter $t$, and set

$$
\Lambda_{t}=\mathbb{Q}(t)\left[h_{1}, h_{2}, h_{3}, \ldots\right] .
$$

The functions $Q_{\lambda}^{\prime}[X ; t]$ are defined as the family of symmetric functions satisfying

$$
Q_{\lambda}^{\prime}[X ; t]=s_{\lambda}+\text { terms of the form } r_{\lambda \mu}(t) s_{\mu} \text { for } \mu>\lambda
$$

and

$$
\left\langle Q_{\lambda}^{\prime}[X ; t], Q_{\mu}^{\prime}[X ; t]\right\rangle_{t}=0 \text { if } \lambda \neq \mu
$$

where the scalar product $\langle\cdot, \cdot\rangle_{t}$ is defined so that

$$
\left\langle p_{\lambda}, p_{\mu}\right\rangle_{t}=z_{\lambda} \delta_{\lambda \mu} \prod_{i}\left(1-t^{\lambda_{i}}\right)
$$


By this definition of the $t$-scalar product, we see that $\left\langle p_{\lambda}, p_{\mu}\right\rangle_{t}=\left\langle p_{\lambda}[X], p_{\mu}[X(1-t)]\right\rangle$, where on the right hand side we have used the usual scalar product from Equation (1.23).

We will mainly be using the scalar product from Equation (1.23), so define $\left\{P_{\lambda}[X ; t]\right\}_{\lambda \vdash n}$ to be the dual basis to the basis $\left\{Q_{\lambda}^{\prime}[X ; t]\right\}_{\lambda \vdash n}$. Since we know that

$$
\left\langle Q_{\lambda}^{\prime}[X ; t], Q_{\mu}^{\prime}[X(1-t) ; t]\right\rangle=0 \quad \text { if } \lambda \neq \mu,
$$

we must have $P_{\lambda}[X ; t]=c_{\lambda} Q_{\lambda}^{\prime}[X(1-t) ; t]$ for some coefficients $c_{\lambda}$. Explicit formulas for the coefficient $c_{\lambda}$ are known, but since

$$
1=\left\langle Q_{\lambda}^{\prime}[X ; t], P_{\lambda}[X ; t]\right\rangle=\left\langle Q_{\lambda}^{\prime}[X ; t], c_{\lambda} Q_{\lambda}^{\prime}[X(1-t) ; t]\right\rangle
$$

it follows that $c_{\lambda}=\left\langle Q_{\lambda}^{\prime}[X ; t], Q_{\lambda}^{\prime}[X(1-t) ; t]\right\rangle^{-1}$.

Example 1.38. We compute the Hall-Littlewood symmetric functions for partitions of 3 using this method to demonstrate how they might be implemented in a computer program.

The triangularity relation shows that $Q_{3}^{\prime}=s_{3}$. Then $Q_{21}^{\prime}$ is defined as

$$
Q_{21}^{\prime}=s_{21}-\frac{\left\langle s_{21}, Q_{3}^{\prime}\right\rangle_{t}}{\left\langle Q_{3}^{\prime}, Q_{3}^{\prime}\right\rangle_{t}} Q_{3}^{\prime}
$$

By calculating from the expansion of the Schur functions in the power sums as in Example 1.37, we have that $\left\langle s_{21}, s_{3}\right\rangle_{t}=t^{2}-t$ and $\left\langle s_{3}, s_{3}\right\rangle_{t}=1-t$. We conclude that $Q_{21}^{\prime}=s_{21}+t s_{3}$.

Similarly we can compute the last Hall-Littlewood symmetric function of size 3 by the computation

$$
Q_{111}^{\prime}=s_{111}-\frac{\left\langle s_{111}, Q_{21}^{\prime}\right\rangle_{t}}{\left\langle Q_{21}^{\prime}, Q_{21}^{\prime}\right\rangle_{t}} Q_{21}^{\prime}-\frac{\left\langle s_{111}, Q_{3}^{\prime}\right\rangle_{t}}{\left\langle Q_{3}^{\prime}, Q_{3}^{\prime}\right\rangle_{t}} Q_{3}^{\prime}
$$

Using the previous examples it is not difficult to compute the scalar products $\left\langle s_{111}, s_{21}\right\rangle_{t}=$ $t^{2}-t,\left\langle s_{111}, s_{3}\right\rangle_{t}=t^{2}-t^{3},\left\langle s_{21}, s_{21}\right\rangle_{t}=(1-t)\left(1-t+t^{2}\right)$. From these computations we compute that

$$
Q_{111}^{\prime}=s_{111}+\left(t+t^{2}\right) s_{21}+t^{3} s_{3} .
$$

There are other ways of computing the Hall-Littlewood symmetric functions. They can also be defined by means of 'creation' operators that generalize the creation operators for the Schur functions. Define

$$
\mathbf{B}_{m}=\sum_{i, j \geq 0}(-1)^{i} t^{j} h_{m+i+j} e_{i}^{\perp} h_{j}^{\perp}=\sum_{j \geq 0} t^{j} \mathbf{S}_{m+j} h_{j}^{\perp} .
$$

This family of operators [66] has the property that if $m \geq \lambda_{1}$,

$$
\mathbf{B}_{m}\left(Q_{\lambda}^{\prime}[X ; t]\right)=Q_{\left(m, \lambda_{1}, \lambda_{2}, \ldots, \lambda_{\ell}\right)}^{\prime}[X ; t] .
$$

These operators will play an important role in one of the definitions of the $k$-Schur functions in Section 3. 
Sage Example 1.39. In SAgE, Example 1.38 can be checked as follows. Note that now we need to define the Schur functions over the base ring of the Hall-Littlewood functions:

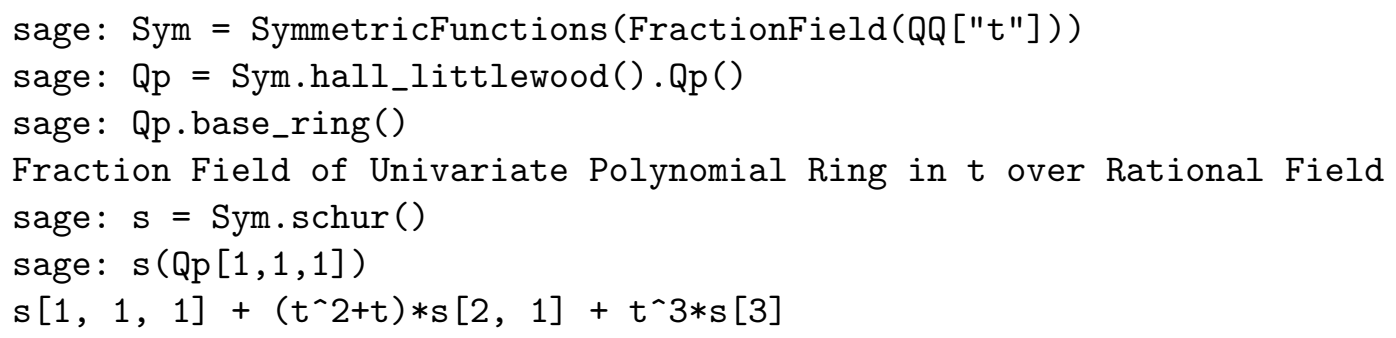

Recall the map $\theta_{q t}(q, t)$ of Equation (1.20) which sends $p_{k} \mapsto p_{k}\left(1-q^{k}\right) /\left(1-t^{k}\right)$. Then we can transform the usual scalar product $\langle\cdot, \cdot\rangle$ to the scalar product for the Hall-Littlewood polynomials $\langle\cdot, \cdot\rangle_{t}$ of $(1.32)$ by setting $t=0$ and replacing $q$ by $t$ in $\theta_{q t}(q, t)$, that is $\theta_{q t}(t, 0)$. We can now check our previous computation for $\left\langle s_{21}, s_{3}\right\rangle_{t}$ in SAGE as follows:

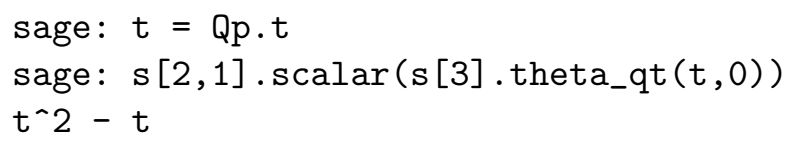

We can also check (1.34):

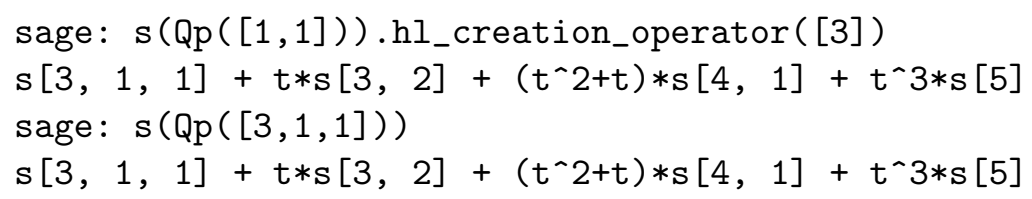

\subsection{Macdonald symmetric functions}

Finally we introduce the Macdonald symmetric functions which form a basis of the space of the symmetric functions with two parameters

$$
\Lambda_{q, t}=\mathbb{Q}(q, t)\left[h_{1}, h_{2}, h_{3}, \ldots\right] .
$$

We provide here a definition of the Macdonald symmetric functions that generalizes those of the Hall-Littlewood and Schur functions introduced in the previous sections. The Macdonald symmetric functions $H_{\lambda}[X ; q, t]$ are defined so that they are the unique basis which has the property that

$$
H_{\lambda}[X ; q, t]=r_{\lambda}(q, t) s_{\lambda}[X /(1-q)]+\text { terms of the form } r_{\lambda \mu}(q, t) s_{\mu}[X /(1-q)]
$$

with $\mu>\lambda$ and

$$
\left\langle H_{\lambda}[X ; q, t], H_{\mu}[X ; q, t]\right\rangle_{q t}=0 \text { if } \lambda \neq \mu
$$


where the scalar product $\langle\cdot, \cdot\rangle_{q t}$ is defined so that

$$
\left\langle p_{\lambda}, p_{\mu}\right\rangle_{q t}=z_{\lambda} \delta_{\lambda \mu} \prod_{i}\left(1-q^{\lambda_{i}}\right)\left(1-t^{\lambda_{i}}\right)
$$

In addition, the condition that $\left\langle H_{\lambda}[X ; q, t], s_{(n)}[X]\right\rangle=t^{n(\lambda)}$ determines the correct scalar multiple of the elements.

Sage Example 1.40. Here we show how to expand the Macdonald symmetric functions in terms of Schur functions for all partitions of 3:

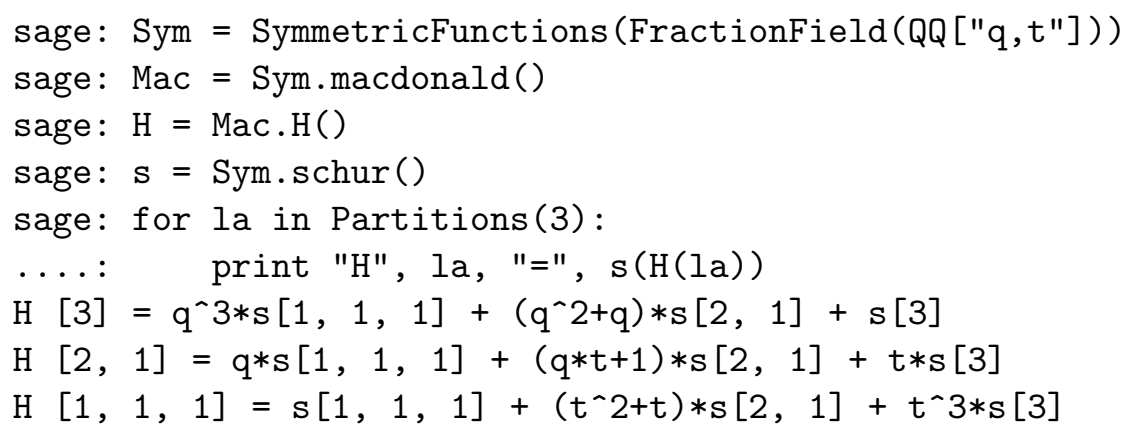

When $q=0$, the expansion is upper triangular with respect to dominance order. In particular, $H_{\lambda}[X ; 0, t]=Q_{\lambda}^{\prime}[X ; t]$.

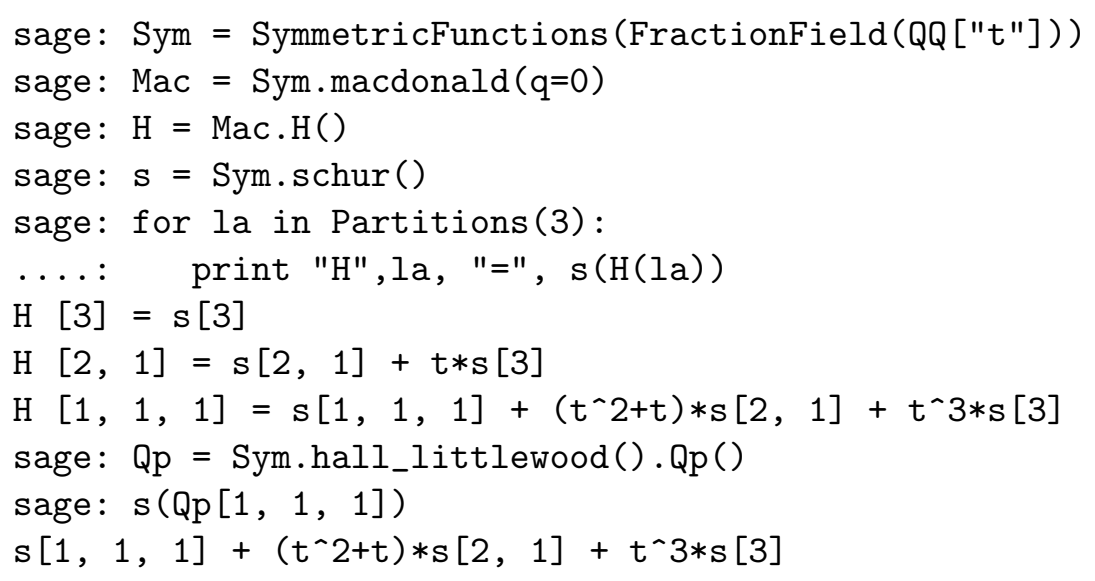

and when $t=0$ it is lower triangular

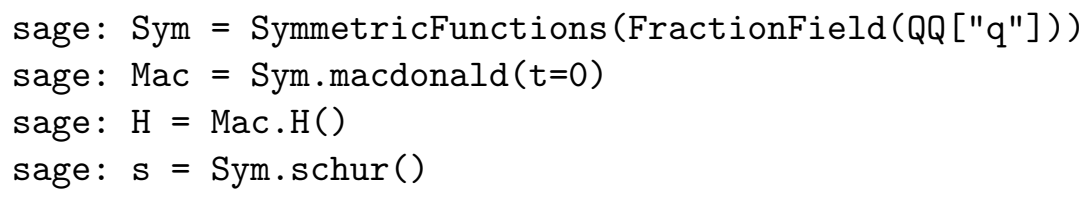




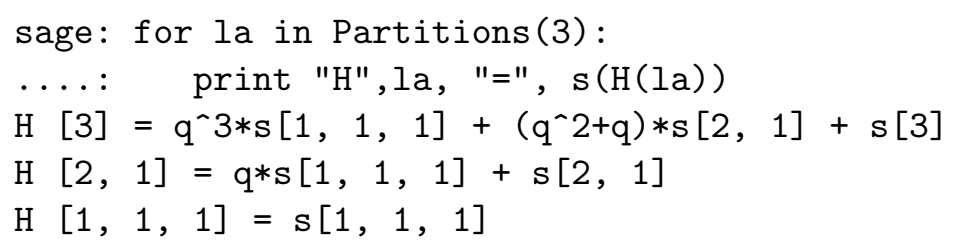

Macdonald [119] introduced the symmetric functions that bear his name in 1988 by expanding on the work of Kevin Kadell (see notes in [120, p. 387]). Attraction to researching Macdonald polynomials grew from conjectures giving combinatorial, geometric, and representation theoretic meaning to the Macdonald/Schur coefficients

$$
K_{\lambda \mu}(q, t):=\left\langle H_{\mu}[X ; q, t], s_{\lambda}\right\rangle,
$$

usually referred to as the Macdonald-Kostka or $q, t$-Kostka coefficients. From the definition that we have presented here, it is not even clear that these coefficients are polynomials in $q$ and $t$. Nevertheless, Macdonald conjectured that they are in fact positive sums of monomials in $q$ and $t$; that is, $K_{\lambda \mu}(q, t) \in \mathbb{N}[q, t]$. These coefficients have since been a matter of great interest.

When $q=0$, the Kostka-Foulkes polynomials $K_{\lambda \mu}(0, t)$ are the Hall-Littlewood/Schur transition coefficients since $H_{\lambda}[X ; 0, t]=Q_{\lambda}^{\prime}[X ; t]$. Kostka-Foulkes polynomials appear in other contexts including affine Kazhdan-Lusztig theory [111] and affine tensor product multiplicities [130, 144]. Moreover, these polynomials encode the dimensions of certain bigraded $S_{n}$-modules [51]. Lascoux and Schützenberger [107] give an intrinsically positive formula for $K_{\lambda \mu}(0, t)$ using tableaux which we now describe.

Recall that a semi-standard tableau is a nested sequence of partitions such that consecutive partitions form a horizontal strip, also identified by a filling of a partition with the integers so that the label of the integer $i$ indicates the cells which are added between the $(i-1)^{s t}$ and $i^{t h}$ partition in the sequence. The horizontal strip condition ensures that the entries increase strictly (resp. weakly) along columns (resp. rows). A semi-standard tableau has weight $\mu$ when there are $\mu_{i}$ labels of $i$. When $\mu$ forms a partition, the tableau is said to have partition weight and when the weight is $(1,1, \ldots, 1)$, the tableau is called standard. A statistic (non-negative integer) called charge, defined in [107], can be associated to each semi-standard tableau. Here, we describe charge for any semi-standard tableaux with partition weight.

First consider the definition of charge on a standard tableau $T$. Define the index $I$ of $T$, starting from $I_{1}=0$, by

$$
I_{r}= \begin{cases}I_{r-1}+1 & \text { if } r \text { is east of } r-1 \\ I_{r-1} & \text { otherwise }\end{cases}
$$

for $r=2, \ldots, n$. The charge of $T$ is the sum of entries in $I(T)$. The notion of charge is easily extended to a generic semi-standard tableau by successively computing the index of an appropriate choice of $i$ cells containing the letters $1,2, \ldots, i$. 
Definition 1.41. From a specific $x$ in cell $c$ of a tableau $T$, the desired choice of $x+1$ is the south-easternmost one lying above $c$. If there are none above $c$, the choice is the south-easternmost $x+1$ in all of $T$.

Consider now any semi-standard tableau $T$ with partition weight. Starting from the rightmost 1 in $T$, use Definition 1.41 to distinguish a standard sequence of $i$ cells containing $1,2, \ldots, i$. Compute the index and then delete all cells in this sequence. Repeat the process on the remaining cells. The total charge is defined to be the sum of all the index vectors.

Example 1.42.

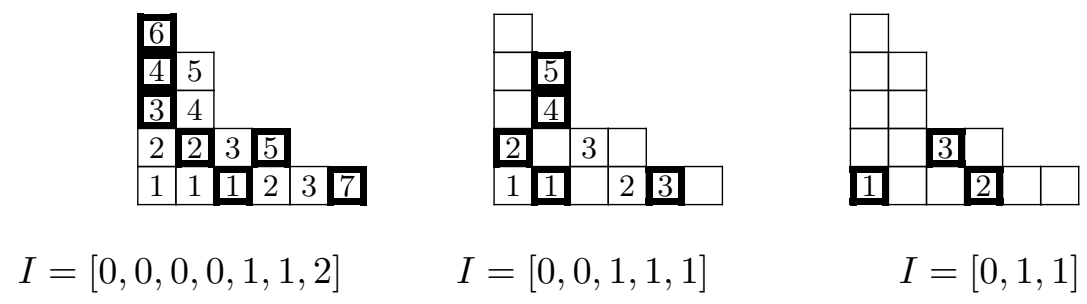

so that the charge is 9 .

We can check this in SAGE:

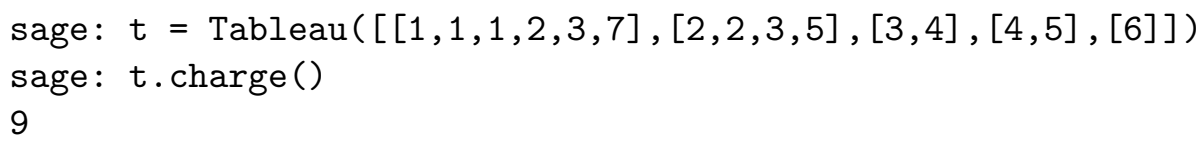

This given, with the shape of a semi-standard tableau $T$ denoted by shape $(T)$ and the charge denoted charge $(T)$, it is proven in [107] that

$$
K_{\lambda \mu}(0, t)=\sum_{\substack{\operatorname{weight}(T)=\mu \\ \text { shape }(T)=\lambda}} t^{\operatorname{charge}(T)} .
$$

Despite having such concrete results for the $q=0$ case, it was a big effort just to establish polynomiality for general $K_{\lambda \mu}(q, t)[53,70,71,100,142]$. The geometry of Hilbert schemes was finally needed to prove positivity [60,61], where Haiman completed his proof by showing that there is a representation theoretical model (often referred to as the " $n$ ! conjecture" [50]) for which these coefficients are formulas for graded multiplicities of occurrences of irreducible representations. A formula in the spirit of (1.38) still remains a mystery.

There are other ways to express the charge. For example in [115] a different formulation of charge is used, which comes from the Ram-Yip formula for Macdonald polynomials [137], and is related to the quantum Bruhat graph, which first arose in connection with the quantum cohomology of the flag variety $[24,49]$. This point of view is dual to the description above in the sense that the positions of the entries in a tableau $T$ are recorded by columns 
$b_{1} \cdots b_{n}$, where $n$ is the value of the largest letter in $T$. Column $b_{i}$ records the column positions of the letters $i$ in $T$, where the columns of $T$ are labelled from right to left.

We attach to $b_{1} \cdots b_{n}$ a reordered filling $c:=c_{1} \cdots c_{n}$ according to the following algorithm, which is based on the circular order $\prec_{i}$ on $[m]$ starting at $i$, namely $i \prec_{i} i+1 \prec_{i}$ $\cdots \prec_{i} m \prec_{i} 1 \prec_{i} \cdots \prec_{i} i-1$. Here $m$ is the width of $T$.

\section{Algorithm 1.43.}

let $c_{1}:=b_{1}$;

for $j$ from 2 to $n$ do

for $i$ from 1 to height of $b_{j}$ do

let $c_{j}(i):=\min \left(b_{j} \backslash\left\{c_{j}(1), \ldots, c_{j}(i-1)\right\}, \prec_{c_{j-1}(i)}\right)$

end do;

end do;

return $c:=c_{1} \ldots c_{n}$.

Then the charge of $T$ is

$$
\operatorname{charge}(T)=\sum_{\gamma \in \operatorname{Des}(c)} \operatorname{arm}(\gamma)
$$

where $\operatorname{Des}(c)$ are all boxes in $c$ which contain a descent with the box directly to the right and $\operatorname{arm}(\gamma)$ is the arm length of the box $\gamma$, that is the number of boxes to the right of $\gamma$.

Example 1.44. Let us consider the tableau $T$ of Example 1.42. Then

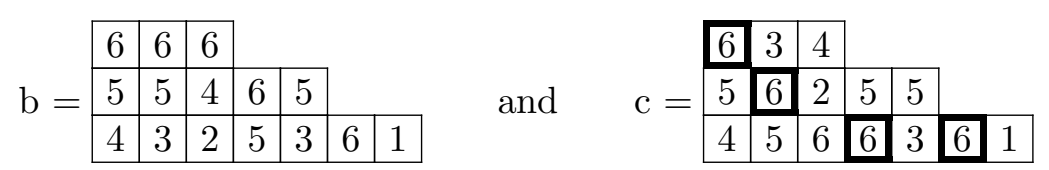

where the boxes with descents are in bold, so that charge $(T)=2+3+3+1=9$.

\subsection{Empirical approach to $k$-Schur functions}

The $k$-Schur functions came out of a study of the $q, t$-Kostka coefficients and this study [92] led to a refinement of Macdonald's positivity conjecture; for any fixed integer $k>0$ and each $\lambda \in \mathcal{P}^{k}$ (a partition where $\lambda_{1} \leq k$ ),

$$
H_{\lambda}[X ; q, t]=\sum_{\mu \in \mathcal{P}^{k}} K_{\mu \lambda}^{(k)}(q, t) A_{\mu}^{(k)}[X ; t] \quad \text { where } \quad K_{\mu \lambda}^{(k)}(q, t) \in \mathbb{N}[q, t]
$$

for some family of polynomials defined by certain sets of tableaux $\mathcal{A}_{\mu}^{k}$ as:

$$
A_{\mu}^{(k)}[X ; t]=\sum_{T \in \mathcal{A}_{\mu}^{k}} t^{\operatorname{charge}(T)} s_{\operatorname{shape}(T)} .
$$


In [92], Lapointe, Lascoux, and Morse conjectured that such $\left\{A_{\mu}^{(k)}[X ; t]\right\}_{\mu_{1} \leq k}$ exists and forms a basis for

$$
\Lambda_{(k)}^{t}=\operatorname{span}\left\{H_{\lambda}[X ; q, t]\right\}_{\lambda_{1} \leq k}
$$

They also conjectured that more remarkably, for any $k^{\prime}>k$,

$$
A_{\lambda}^{(k)}[X ; t]=\sum_{\mu} B_{\lambda, \mu}^{\left(k, k^{\prime}\right)}(t) A_{\mu}^{\left(k^{\prime}\right)}[X ; t] \quad \text { where } B_{\lambda, \mu}^{\left(k, k^{\prime}\right)}(t) \in \mathbb{N}[t]
$$

Given that $A_{\mu}^{(k)}[X ; t]=s_{\mu}$ for $k \geq|\mu|$, the decomposition (1.39) strengthens Macdonald's conjecture. Although these new bases arose in the context of Macdonald polynomials, pursuant work led to unexpected connections with geometry, physics, and representation theory. At the root, $\left\{A_{\mu}^{(k)}[X ; t]\right\}$ generalizes the very aspects of the Schur basis that make it so fundamental and wide-reaching. As such, the functions are called $k$-Schur functions.

Before we give any formal definition for $k$-Schur functions, we begin with some computational examples that demonstrate how this all came about.

Example 1.45. Consider the Hall-Littlewood symmetric functions that are spanned by partitions of 3 and 4 with $\lambda_{1} \leq 2$ :

$$
\begin{aligned}
Q_{111}^{\prime}[X ; t] & =s_{111}+\left(t+t^{2}\right) s_{21}+t^{3} s_{3} \\
Q_{21}^{\prime}[X ; t] & =s_{21}+t s_{3}
\end{aligned}
$$

and

$$
\begin{aligned}
Q_{1111}^{\prime}[X ; t] & =s_{1111}+\left(t+t^{2}+t^{3}\right) s_{211}+\left(t^{2}+t^{4}\right) s_{22}+\left(t^{3}+t^{4}+t^{5}\right) s_{31}+t^{6} s_{4} \\
Q_{211}^{\prime}[X ; t] & =s_{211}+t s_{22}+\left(t+t^{2}\right) s_{31}+t^{3} s_{4} \\
Q_{22}^{\prime}[X ; t] & =s_{22}+t s_{31}+t^{2} s_{4} .
\end{aligned}
$$

The first clue of the existence of 2-Schur functions is to notice that there is a set of symmetric functions $A_{\lambda}^{(2)}[X ; t]$ for $\lambda \vdash 3,4$ such that $\lambda_{1} \leq 2$ and

- they form a basis for this subset of Hall-Littlewood symmetric functions that have coefficients that are non-negative polynomials in $t$ when expanded in the Schur basis;

- when expanded in the Hall-Littlewood basis have a term $Q_{\lambda}^{\prime}[X ; t]$ and all other terms are larger in dominance order;

- have a leading term in the Schur basis which is $s_{\lambda}$ and are in the linear span of Schur functions indexed by partitions which are larger than $\lambda$ in dominance order;

- if the involution $\omega$ is applied and $t$ is replaced by $1 / t$, then the 2-Schur function is equal to another 2-Schur function up to a power of $t$; 
- the Schur function indexed by the partition which is largest in dominance order is a power of $t$ times $s_{\left(\lambda^{\omega}\right)^{\prime}}$ (indexed by the conjugate of the $k$-conjugate of $\lambda$ );

- for which the Macdonald symmetric functions are positive when expressed in terms of these elements (have coefficients which are polynomials in $q$ and $t$ with non-negative integer coefficients).

It turns out that these conditions are enough to characterize the 2-Schur functions. The triangularity conditions with respect to the Hall-Littlewood polynomials and the Schur functions require that $A_{22}^{(2)}[X ; t]=s_{22}+t s_{31}+t^{2} s_{4}$. Then again by triangularity and positivity we have $A_{211}^{(2)}[X ; t]=s_{211}+t s_{31}$, and finally $A_{1111}^{(2)}[X ; t]=s_{1111}+t s_{211}+t^{2} s_{22}$.

Sage Example 1.46. The conditions involving the $k$-conjugate of the partition can easily be checked

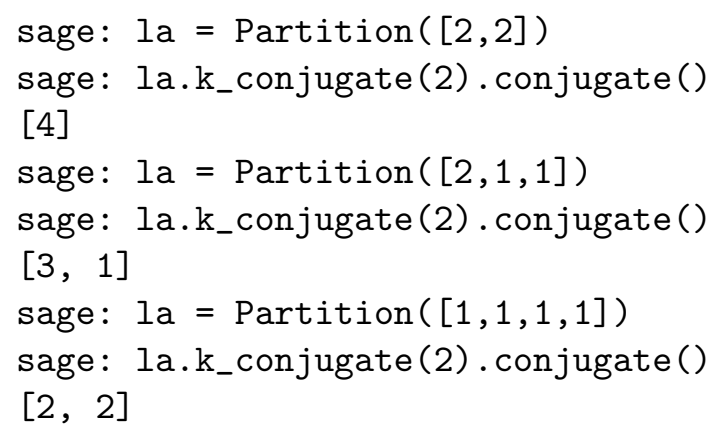

as well as the statement about the application of $\omega$ composed with $t \mapsto 1 / t$ :

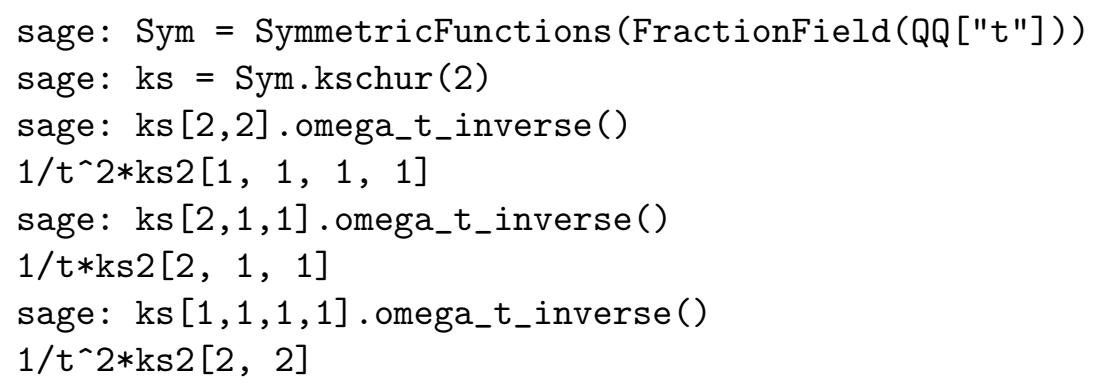

We can also check the positive expansion of the Macdonald polynomials:

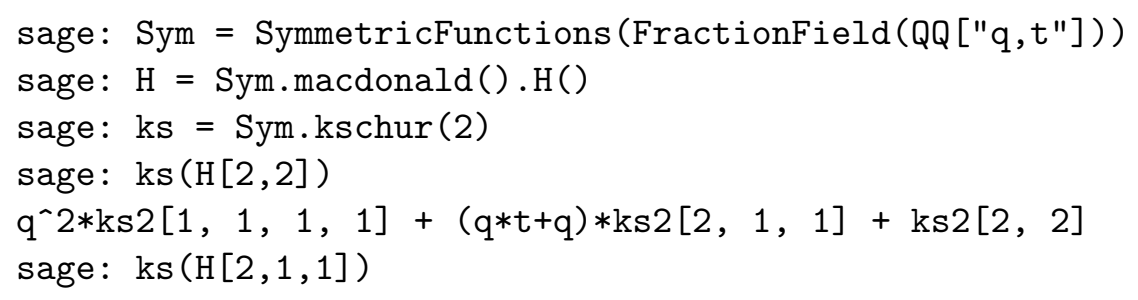




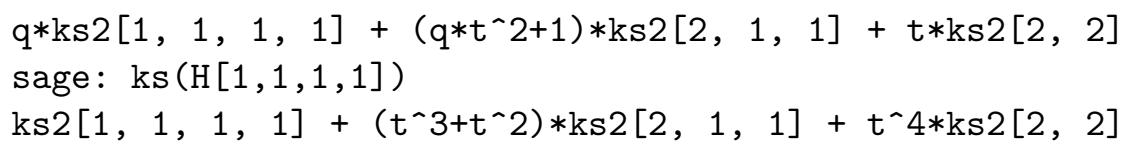

At $k=3$, the above conditions are no longer a complete characterization of the $k$-Schur functions and it is difficult to guess what $A_{\lambda}^{(3)}$ is for some of the partitions at $\lambda \vdash 7$. We add as a last condition that the $k$-Schur functions should be the 'smallest' basis with the above properties. The existence of such a basis alone is enough to recognize that there is something remarkable about the $k$-Schur functions, because it is unusual to see a basis for which the Macdonald symmetric functions expand positively.

Example 1.47. At $k=3$, the triangularity condition for the Hall-Littlewood basis implies that,

$$
\begin{aligned}
A_{331}^{(3)}[X ; t]=Q_{331}^{\prime}[X ; t]=s_{331} & +t s_{421}+\left(t+t^{2}\right) s_{43}+t^{2} s_{511}+\left(t^{2}+t^{3}\right) s_{52} \\
& +\left(t^{3}+t^{4}\right) s_{61}+t^{5} s_{7}
\end{aligned}
$$

and since

$$
\begin{aligned}
Q_{322}^{\prime}[X ; t]=s_{322} & +t s_{331}+\left(t+t^{2}\right) s_{421}+\left(t^{2}+t^{3}\right) s_{43}+t^{3} s_{511} \\
& +\left(t^{2}+t^{3}+t^{4}\right) s_{52}+\left(t^{4}+t^{5}\right) s_{61}+t^{6} s_{7}
\end{aligned}
$$

we can use the triangularity and positivity properties from above that imply $A_{322}^{(3)}[X ; t]$ must be given by

$$
A_{322}^{(3)}[X ; t]=Q_{322}^{\prime}[X ; t]-t Q_{331}^{\prime}=s_{322}+t s_{421}+t^{2} s_{52} .
$$

However, to try to determine $A_{3211}^{(3)}[X ; t]$, we calculate

$$
\begin{aligned}
Q_{3211}^{\prime}[X ; t]=s_{3211} & +t s_{322}+\left(t+t^{2}\right) s_{331}+t s_{4111}+\left(t+t^{2}+t^{3}\right) s_{421} \\
& +\left(t^{2}+t^{3}+t^{4}\right) s_{43}+\left(t^{2}+t^{3}+t^{4}\right) s_{511}+\left(2 t^{3}+t^{4}+t^{5}\right) s_{52} \\
& +\left(t^{4}+t^{5}+t^{6}\right) s_{61}+t^{7} s_{7}
\end{aligned}
$$

and it is difficult to tell from the conditions above whether $A_{3211}^{(3)}$ should be $Q_{3211}^{\prime}[X ; t]-$ $t^{2} Q_{331}^{\prime}[X ; t]$ or $Q_{3211}^{\prime}[X ; t]-t Q_{322}^{\prime}[X ; t]$ or some other linear combination of terms.

We leave it as an exercise for the reader to calculate $A_{111}^{(2)}[X ; t], A_{21}^{(2)}[X ; t]$ from (1.42), using the properties in Example 1.45 as a characterization. With the additional symmetric function $Q_{31}^{\prime}[X ; t]=s_{31}+t s_{4}$, it is a worthwhile exercise to determine the symmetric functions $A_{31}^{(3)}[X ; t], A_{22}^{(3)}[X ; t], A_{211}^{(3)}[X ; t]$ and $A_{1111}^{(3)}[X ; t]$ to see how it might be possible to define the $k$-Schur functions for small values by experimentation. 
Once it is clear that these symmetric functions exist, it is a matter of determining an algorithm or a formula for computing them. It was along these lines that Lapointe, Lascoux, and Morse discovered $k$-Schur functions and their first formula appeared in [93] where a method is given for constructing the sets $\mathcal{A}_{\lambda}$ in (1.40). Subsequently, various conjecturally equivalent definitions have arisen, each having different benefits and detriments. In the following section we present a construction for $k$-Schur functions when the parameter $t$ is set to one and the parameter case is then addressed in Section 3.

\subsection{Notes on references}

The combinatorics of affine permutations can be expressed in terms of combinatorial models other than $k$-bounded partitions and $(k+1)$-cores such as abaci, windows, codes, and $k$ castles $[12,21,38,41,103]$. Certain combinatorial aspects of $k$-Schur functions are best expressed in terms of $k$-bounded partitions whereas others are better suited to $(k+1)$-cores or affine permutations. It could be that other formulations for the index set are well suited for expressing properties which have not yet been discovered.

For symmetric function notation we generally follow the notation of Macdonald [120] with the addition of the use of plethystic notation (see Equation (1.19)) to encode certain transformations of alphabets. The scalar product $\langle\cdot, \cdot\rangle_{t}$ defined in Section 1.7 and $\langle\cdot, \cdot\rangle_{q t}$ defined in Section 1.8 are not the scalar products that are used in [120], but they are the scalar products needed in order to define the Hall-Littlewood $Q^{\prime}$-basis and the Macdonald $H$-basis that we will use in subsequent sections. Macdonald does not use the $H$-basis in [120], however it is a transformation of the basis referred to as the integral basis (the $J$-basis) and they are related by the transformation $J_{\lambda}[X ; q, t]=H_{\lambda}[X(1-t) ; q, t]=$ $\theta_{t 0}\left(H_{\lambda}[X ; q, t]\right)$ (see, for instance, [50, Eq. I.16]).

The operators $\mathbf{S}_{m}$ defined in Equation (1.30) are usually referred to as Bernstein operators. They first appear in [160, p. 69] (see also [120, Example 29 Section I.5]). Their generalizations to creation operators for Hall-Littlewood symmetric functions is due to Jing [66] (see also [120, Example 8 Section III.5]).

\section{From Pieri rules to $k$-Schur functions at $t=1$}

We first present the definition of the Schur functions as a generating function for semistandard tableaux. This presentation provides the context to show how the $k$-Schur functions and the dual $k$-Schur functions both generalize this definition of the Schur functions. We carefully explain how the algebraic Pieri rule naturally gives rise to distinguished sequences of partitions and show how the combinatorics of 'weak' and 'strong' tableaux captures these relations. 


\subsection{Semi-standard tableaux and a monomial expansion of Schur func- tions}

Recall that the Schur functions satisfy the Pieri rule for multiplication of a Schur function by a homogeneous symmetric function

$$
h_{1} s_{\lambda}=\sum_{\mu: \lambda \rightarrow \mu} s_{\mu}
$$

and more generally,

$$
h_{r} s_{\lambda}=\sum_{\mu} s_{\mu}
$$

where the sum is over all partitions $\mu$ where $\lambda \subseteq \mu,|\mu|=|\lambda|+r$ and $\mu / \lambda$ is a horizontal strip.

Consider how we can use these rules now to expand a homogeneous symmetric function in terms of the Schur basis. A given $h_{\lambda}=h_{\lambda_{1}} h_{\lambda_{2}} \cdots h_{\lambda_{\ell}}$ may be seen as a sequence of operators that act on $1=s_{\emptyset}$ which we act on, first by $h_{\lambda_{1}}$, then $h_{\lambda_{2}}$, and so on. We demonstrate this with the following example.

Example 2.1. We expand the expression $h_{431}$ in terms of Schur functions by successive applications of the Pieri rule:

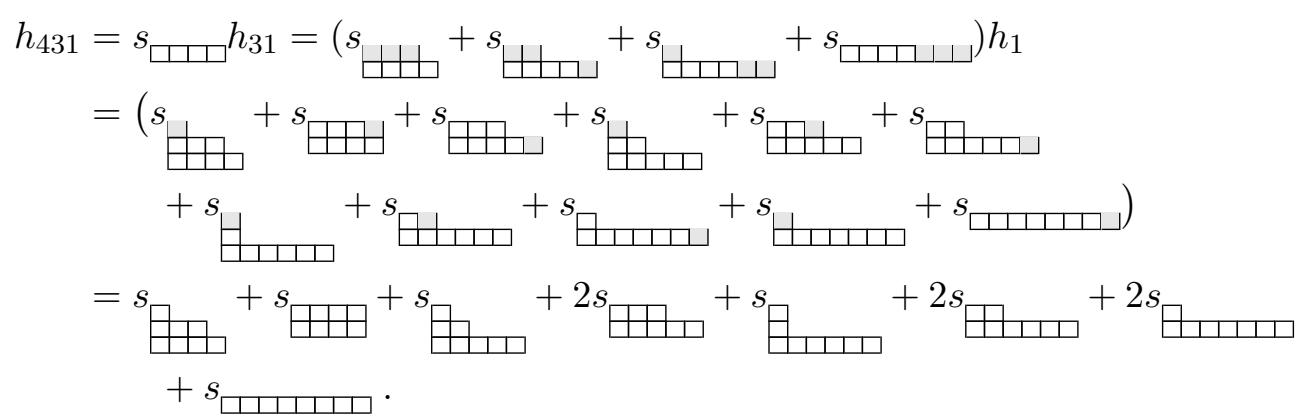

Notice in the example that every term in the expansion of the product of $h$ 's is represented by a sequence of partitions which records how that term appears in the final expression. For instance there are two terms representing $(5,3)$, one that arose from the sequence $\emptyset \subseteq(4) \subseteq(5,2) \subseteq(5,3)$ and the other which arose from the sequence $\emptyset \subseteq(4) \subseteq(4,3) \subseteq(5,3)$. A sequence

$$
\lambda^{(0)} \subseteq \lambda^{(1)} \subseteq \cdots \subseteq \lambda^{(d)},
$$

where each $\lambda^{(i+1)} / \lambda^{(i)}$ for $0 \leq i<d$ is a horizontal strip, is called a semi-standard skew tableau. When $\lambda^{(0)}$ is empty, the sequence is called a semi-standard tableau (see also 
Section 1.8). We say that the shape of the tableau is $\lambda^{(d)} / \lambda^{(0)}\left(\right.$ or $\lambda^{(d)}$ if $\lambda^{(0)}=\emptyset$ ) and the weight of the tableau is the sequence

$$
\left(\left|\lambda^{(1)} / \lambda^{(0)}\right|,\left|\lambda^{(2)} / \lambda^{(1)}\right|, \ldots,\left|\lambda^{(d)} / \lambda^{(d-1)}\right|\right) .
$$

Note that for any partition there is precisely one semi-standard tableau that has both shape and weight equal to $\lambda$. If $\lambda$ and $\mu$ are both partitions of the same size, then there is at least one partition of shape $\lambda$ and weight $\mu$ if and only if $\lambda \geq \mu$.

A semi-standard tableau of shape $\lambda$ is usually thought of as a filling of the partition diagram for $\lambda$ by placing a 1 in each of the cells of $\lambda^{(1)} / \lambda^{(0)}$, a 2 in each of the cells of $\lambda^{(2)} / \lambda^{(1)}$, and more generally each of the cells of $\lambda^{(i)} / \lambda^{(i-1)}$ is labelled with an $i$. Note, iteration of the special case (2.1) ensures that each $\lambda^{(i)} / \lambda^{(i-1)}$ contains exactly one cell. These are the tableaux of weight $(1,1, \ldots, 1)$ and they are called standard tableaux.

Example 2.2. The coefficient of $s_{52}$ in $h_{421}$ is equal to 2 . The reason for this is that one term comes from

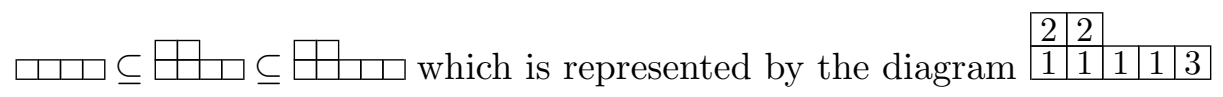

and the other comes from

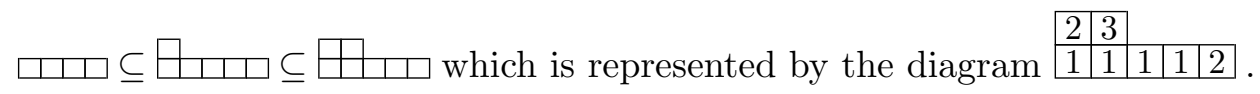

Remark 2.3. Another useful alternative to the conventional definition of tableaux is to instead define a semi-standard tableau to be a standard tableau with certain conditions on its reading word. The reading word of a tableau is obtained by taking the entries of $T$ from top to bottom and left to right. A tableau of weight $\alpha$ is then a standard tableau having increasing reading words in the alphabets

$$
\mathcal{A}_{\alpha, x}=\left[1+\Sigma^{x-1} \alpha, \Sigma^{x} \alpha\right] \quad \text { where } \quad \Sigma^{x} \alpha=\sum_{i \leq x} \alpha_{i},
$$

for each $x=1, \ldots, \ell(\alpha)$. For example, this observation is central in the study of quasisymmetric functions.

It is typical to represent the number of semi-standard tableaux of shape $\lambda$ and weight $\mu$ by the symbol $K_{\lambda \mu}$. These numbers are often referred as the Kostka coefficients. Based on the Pieri rule and a relatively straightforward proof by induction, we can generalize roughly what we see in the example, namely that for $\mu \vdash m$,

$$
h_{\mu}=\sum_{\lambda \vdash m} K_{\lambda \mu} s_{\lambda}
$$


From this, the monomial expansion of a Schur function can be derived. In particular, because $\left\langle s_{\lambda}, s_{\mu}\right\rangle=\delta_{\lambda \mu}$, we can conclude that $\left\langle h_{\mu}, s_{\lambda}\right\rangle=K_{\lambda \mu}$. The coefficient in the monomial expansion of a Schur function then pops out by the duality $\left\langle m_{\lambda}, h_{\mu}\right\rangle=\delta_{\lambda \mu}$ :

$$
s_{\lambda}=\sum_{\mu} d_{\mu \lambda} m_{\mu} \quad \text { where } \quad d_{\mu \lambda}=\left\langle h_{\mu}, \sum_{\alpha} d_{\alpha \lambda} m_{\alpha}\right\rangle=\left\langle h_{\mu}, s_{\lambda}\right\rangle=K_{\lambda \mu}
$$

This formula provides us with a combinatorial expansion of the Schur functions in the monomial basis. We can also derive a formula in terms of variables. Note that the construction of tableaux with weight $\alpha$ applies for any composition $\alpha$. Let $\operatorname{SSYT}(\lambda, \alpha)$ be the set of tableaux with weight $\alpha$ and shape $\lambda$. There is an involution $[10,110]$ on this set that maps a tableau of weight $\alpha$ to a tableau whose weight is a permutation of $\alpha$. Thus, given that $K_{\lambda \alpha}=K_{\lambda \sigma(\alpha)}$,

$$
s_{\lambda}\left[X_{m}\right]=\sum_{T} \mathbf{x}^{T}
$$

where the sum is over all possible semi-standard tableaux of shape $\lambda$ with entries in $\{1,2, \ldots, m\}$. Here $\mathbf{x}^{T}$ denotes $x_{1}^{\alpha_{1}} x_{2}^{\alpha_{2}} \cdots x_{m}^{\alpha_{m}}$, where $\alpha$ is the weight of $T$.

Sage Example 2.4. We now demonstrate on how to produce all semi-standard tableaux of a given shape and weight

$$
\begin{aligned}
& \text { sage: SemistandardTableaux }([5,2],[4,2,1]) \text {. list }() \\
& {[[[1,1,1,1,2],[2,3]],[[1,1,1,1,3],[2,2]]]}
\end{aligned}
$$

The Kostka matrix can be computed as follows:

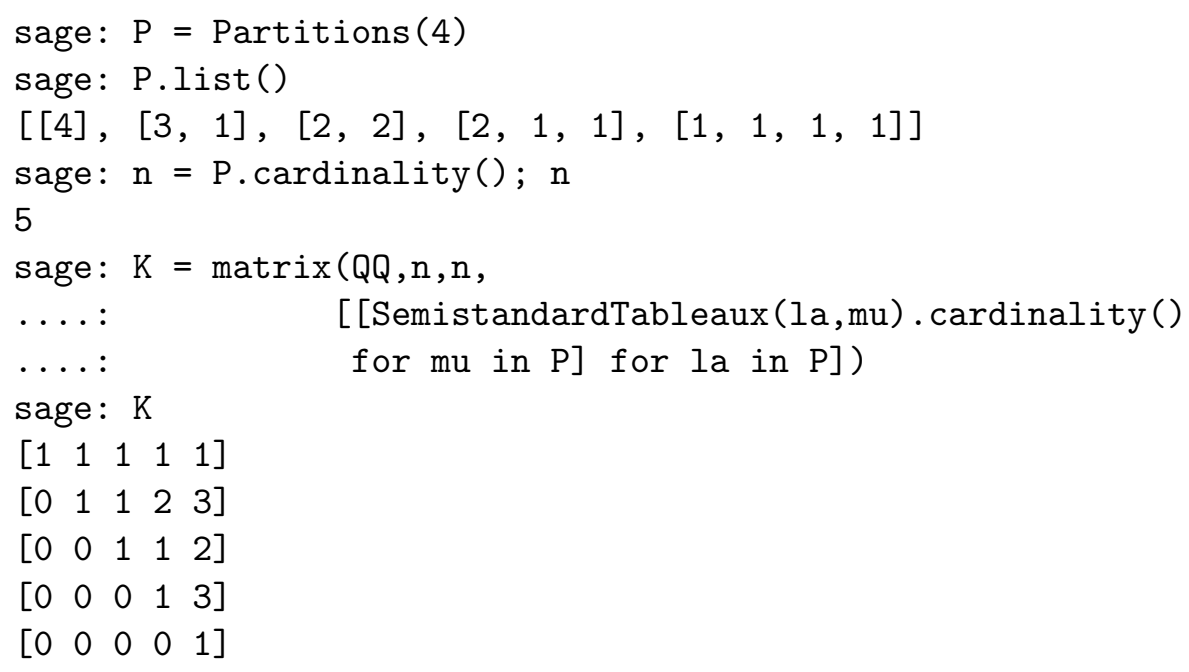

The self duality of the Schur functions is a very remarkable property. In fact, the self duality and a condition on triangularity can be taken as either the defining property for 
Schur functions or as property which easily follows from the definition. It is this duality that implies that $\left\langle h_{\mu}, s_{\lambda}\right\rangle$ will be the coefficient of $s_{\lambda}$ in the expansion of $h_{\mu}$. In the next two sections we shall introduce two bases of a subalgebra/quotient algebra which follows from ideas in this construction.

\subsection{Weak tableaux and a monomial expansion of dual $k$-Schur functions}

We will present the $k$-Schur functions as a basis of the space

$$
\Lambda_{(k)}=\mathbb{Q}\left[h_{1}, h_{2}, h_{3}, \ldots, h_{k}\right] .
$$

It will develop that this basis has a role in $\Lambda_{(k)}$ that in many ways is analogous to the role of the Schur basis in the study of the symmetric function space $\Lambda$. The algebra of $\Lambda_{(k)}$ is no longer a self dual Hopf algebra. The algebra is dual however to a quotient of the ring of symmetric functions. We define

$$
\Lambda^{(k)}=\Lambda /\left\langle m_{\lambda}: \lambda_{1}>k\right\rangle
$$

A basis of $\Lambda_{(k)}$ are the elements $h_{\lambda}$ for partitions $\lambda$ where $\lambda_{1} \leq k$. The elements $m_{\lambda}$ with $\lambda_{1} \leq k$ may be chosen as representatives of the dual algebra. We will present two bases: the $k$-Schur functions $s_{\lambda}^{(k)}$ which form a basis for $\Lambda_{(k)}$ and the dual k-Schur functions $\tilde{F}_{\lambda}^{(k)}$ which are representative elements of the basis for the dual algebra $\Lambda^{(k)}$. We proceed by defining the basis of $k$-Schur functions and the other will be determined by duality.

Recall by Proposition 1.3 that the $k$-bounded partitions of size $m$ are in bijection with the $(k+1)$-cores of length $m$ (or equivalently cores with $m$ cells with hook length of size less than or equal to $k$ ). At this point, we keep in mind that the $k$-bounded partition $\lambda$ that serves as an index for either a $k$-Schur function or a dual $k$-Schur function represents the shape of a $k+1$-core, $\mathfrak{c}(\lambda)$. Sometimes we will use the properties of the partition $\lambda$ and other times we will use the corresponding core, $\mathfrak{c}(\lambda)$. Later, we will interpret things in the language of $k$-bounded partitions and affine Grassmannian elements.

We will define the $k$-Schur functions at $t=1$ based on a weak Pieri rule for $k$-Schur functions (which at this point exists only as data on the computer because we deduced what it must be from the atom definition in the last chapter). This is the relation, for a $k$-bounded partition $\mu$ and $r \leq k$,

$$
h_{r} s_{\mu}^{(k)}=\sum_{\lambda} s_{\lambda}^{(k)}
$$

summing over $k$-bounded partitions $\lambda$ where $\mathfrak{c}(\lambda) / \mathfrak{c}(\mu)$ is a weak horizontal strip of size $r$ (see Equation (1.5)).

Example 2.5. With $k=3$, to compute $h_{1} s_{31}^{(3)}$, we find all 4-cores that cover $\mathfrak{c}(3,1)=(4,1)$ in the weak order poset Figure 2.3 and there are two, one of shape $\mathfrak{c}(3,2)=(5,2)$ and one 
of shape $\mathfrak{c}(3,1,1)=(4,1,1)$ :

$$
h_{1} s_{31}^{(3)}=s_{32}^{(3)}+s_{311}^{(3)} .
$$

To compute $h_{2} s_{31}^{(3)}$, we follow the weak horizontal chains of length 2 from $(4,1)$ in Figure 2.3 and notice that there are two of shape $\mathfrak{c}(3,3)=(6,3)$ and $\mathfrak{c}(3,2,1)=(5,2,1)$ :

$$
h_{2} s_{31}^{(3)}=s_{33}^{(3)}+s_{321}^{(3)} .
$$

Note that there is a length 2 chain from $\mathfrak{c}(3,1)=(4,1)$ to $\mathfrak{c}(3,1,1,1)=(4,1,1,1)$, but because $(4,1,1,1) /(4,1)$ is not a weak horizontal strip, this term is omitted. There is only one horizontal chain of length 3 from $\mathfrak{c}(3,1)=(4,1)$ implying that

$$
h_{3} s_{31}^{(3)}=s_{331}^{(3)} .
$$

As we have shown for usual Schur functions, the iteration of the Pieri rule can be used to inspire a family of tableaux where, in this case, their enumeration gives the coefficient of $s_{\lambda}^{(k)}$ in the expansion of $h_{\mu} s_{\emptyset}^{(k)}$. Consider the following example.

Example 2.6. For $k=4$, we compute $h_{431}$ in terms of $k$-Schur functions. We will index the $k$-Schur function by a diagram for a 5 -core with the added cells indicated by an $*$. The indexing 4-bounded partition can be read off of these diagrams by counting only the non-grey cells in each row.

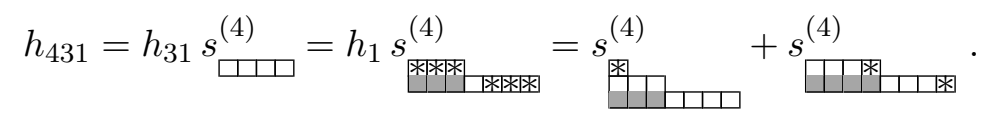

Let $k=6$ and compute an example with more terms:

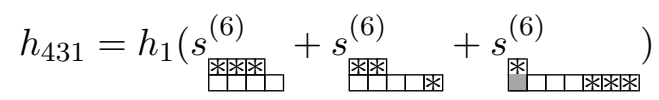

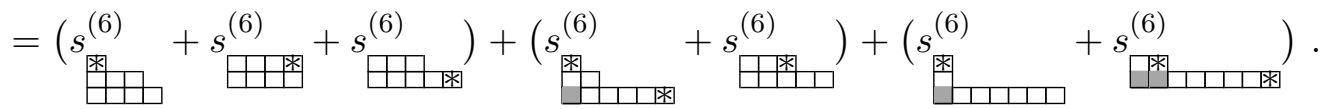

The iteration imposes the conditions needed to characterize a weak tableau; it must be a sequence of $(k+1)$-cores,

$$
\emptyset=\kappa^{(0)} \subseteq \kappa^{(1)} \subseteq \cdots \subseteq \kappa^{(d)}=\mathfrak{c}(\lambda)
$$

such that $\kappa^{(i)} / \kappa^{(i-1)}$ is a weak horizontal strip. We say that its shape is $\mathfrak{c}(\lambda)$ and define its weight $\alpha$ as we did in Equation (2.4), but now using the size function on cores;

$$
\alpha_{i}=\left|\kappa^{(i)} / \kappa^{(i-1)}\right|_{k+1}, \quad \text { for } i=1, \ldots, d .
$$

Let us emphasize that an entry $\alpha_{i}$ of the weight does not record the number of times letter $i$ appears in the tableau. In fact, using (1.8), we see that instead, $\alpha_{i}$ records the number of distinct residues used to label the cells of $\kappa^{(i)} / \kappa^{(i-1)}$. We can more concisely describe weak tableaux in the following terms: 
Proposition 2.7. [95] Let $\kappa$ be a $(k+1)$-core and let $\alpha=\left(\alpha_{1}, \ldots, \alpha_{d}\right)$ be a composition of $|\kappa|_{k+1}$ with no part larger than $k$. A weak tableau of weight $\alpha$ is a semi-standard filling of shape $\kappa$ with letters $1, \ldots, d$ such that the collection of cells filled with letter $i$ is labeled by exactly $\alpha_{i}$ distinct $(k+1)$-residues.

Example 2.8. For $k=6$, the weak tableaux of weight $(4,3,1)$ are

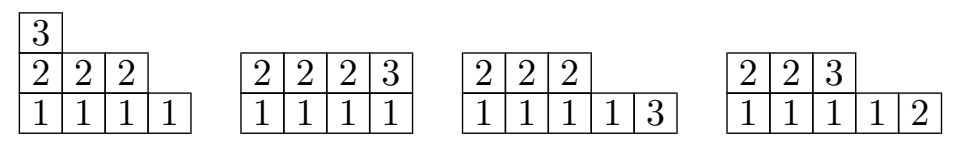

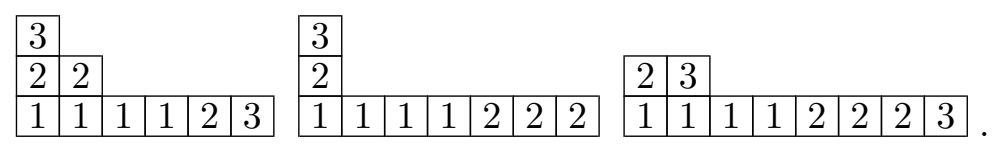

For $k=3$, we list all weak tableaux of shape $(5,2,1)$ which can be extracted by looking at all successions of horizontal chains (with non-increasing sizes) in Figure 2.3.

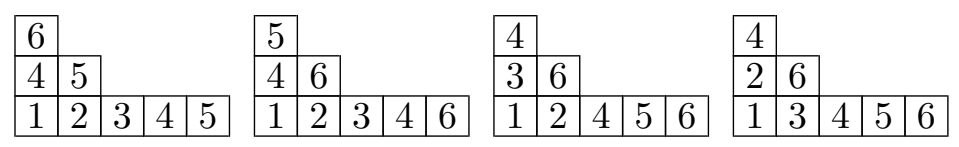

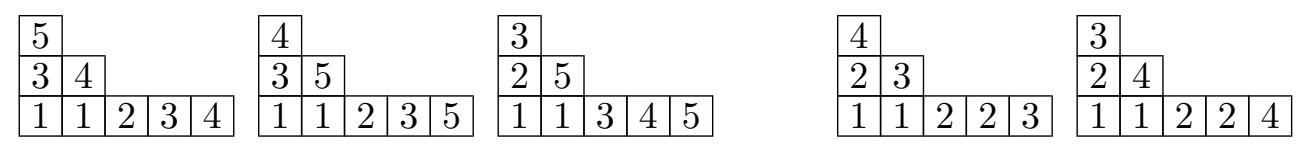

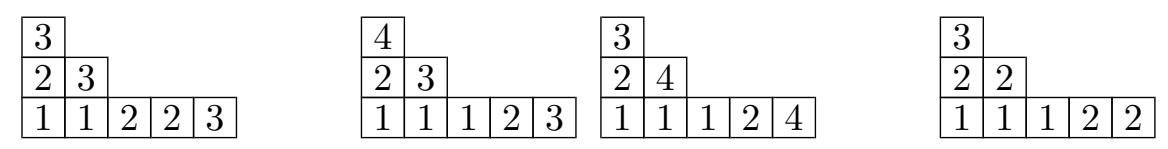

Sage Example 2.9. In SAGE we can list all weak $k$-tableaux for a given shape and weight. For example, the two weak 6 -tableaux of weight $(4,3,1)$ and shape $(5,3)$ can be obtained as follows:

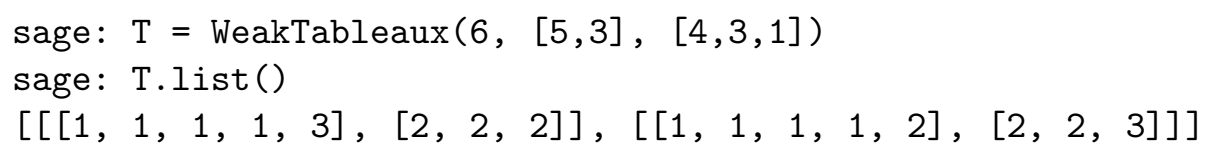

The 13 weak 3 -tableaux of shape $(5,2,1)$ in Example 2.8 can be obtained as

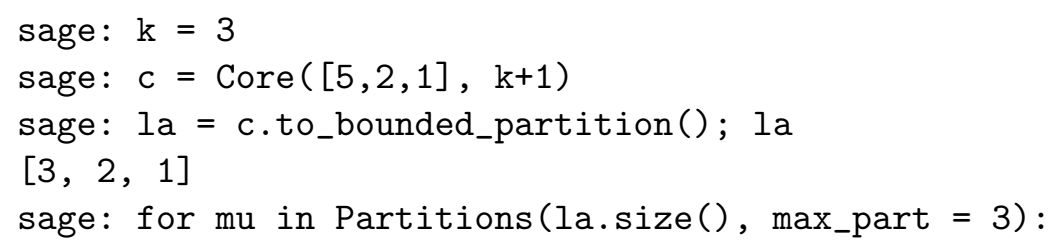




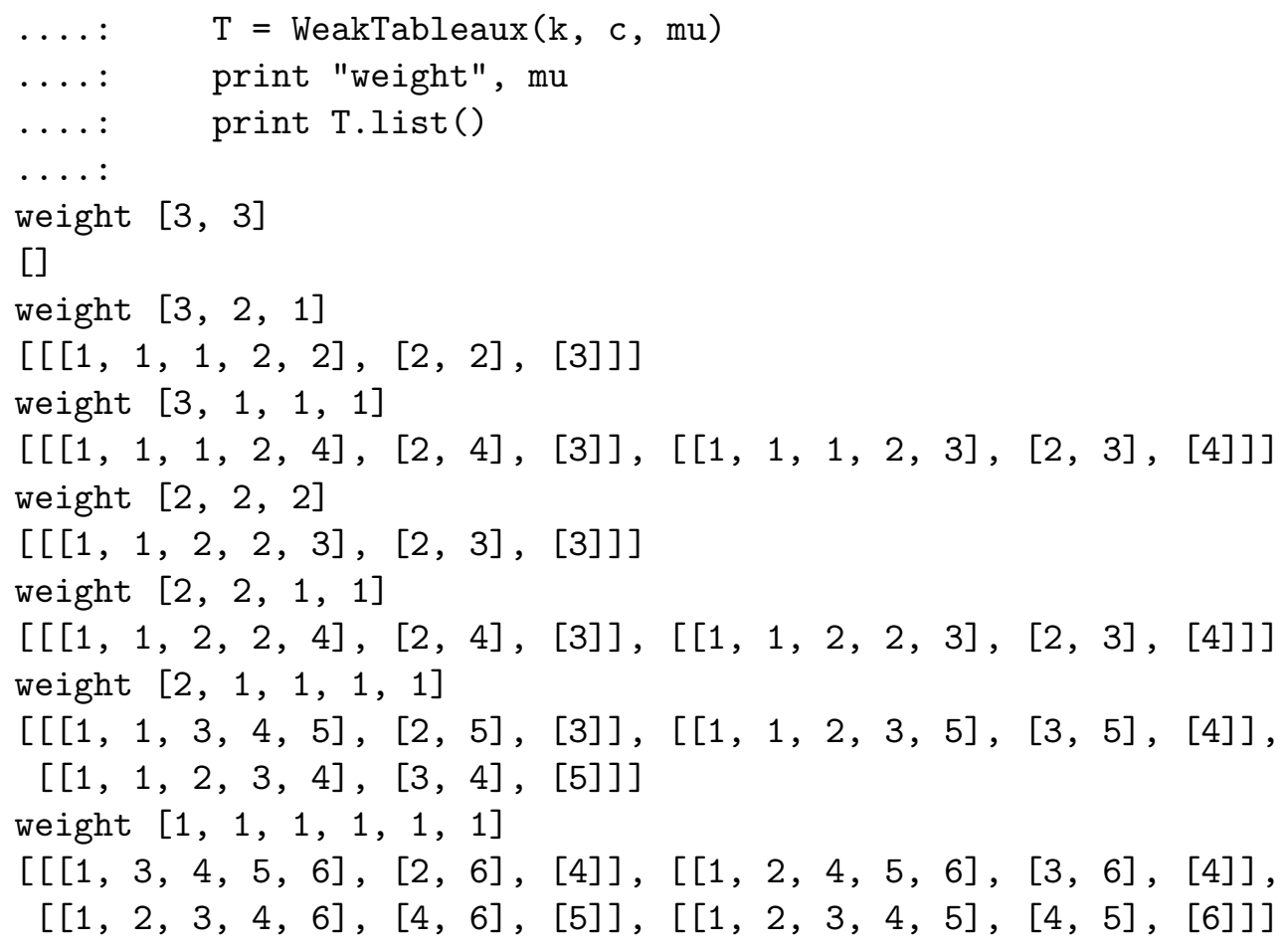

For any $k$-bounded partition $\lambda$ and $k$-bounded partition $\mu$, we denote the number of weak tableaux of shape $\mathfrak{c}(\lambda)$ and weight $\mu$ by $K_{\lambda \mu}^{(k)}$. These numbers, called (weak) $k$-Kostka coefficients, satisfy an important property

$$
K_{\lambda \mu}^{(k)}= \begin{cases}1 & \text { if } \lambda=\mu \\ 0 & \text { if } \lambda \geq \mu,\end{cases}
$$

with respect to dominance order on partitions. Thus, the matrix of coefficients $\left\|K_{\lambda \mu}^{(k)}\right\|$ over all $k$-bounded partitions $\lambda$ and $\mu$ of the same size is unitriangular and thus invertible. It is with this in hand that we arrive at a family of functions that satisfy the weak Pieri rule. To be precise, $k$-Schur functions were characterized in [96] by the system obtained by taking

$$
h_{\mu}=\sum_{\lambda: \lambda_{1} \leq k} K_{\lambda \mu}^{(k)} s_{\lambda}^{(k)}
$$

for all $k$-bounded partitions $\mu$. In fact, this system defines the $k$-Schur basis $\left\{s_{\lambda}^{(k)}\right\}_{\lambda_{1} \leq k}$ because the elements $h_{\lambda}$ for $\lambda_{1} \leq k$ form a basis for the space $\Lambda_{(k)}$ and the transition matrix is invertible over the integers.

Example 2.10. For $k=6$, the weak tableaux in Example 2.8 tell us that

$$
h_{431}=s_{431}^{(6)}+s_{44}^{(6)}+2 s_{53}^{(6)}+s_{521}^{(6)}+s_{611}^{(6)}+s_{62}^{(6)} .
$$


In Section 3, various different notions of $k$-Schur functions will be given. We will work with a running example and do a computation to give an idea of how each of the notions can be implemented and to demonstrate their relative difficulty.

Example 2.11. Let us calculate $s_{3211}^{(3)}$ in terms of homogeneous symmetric functions. The $k$-Schur function $s_{\lambda}^{(k)}$ can be computed recursively using the weak Pieri rule. The product $h_{\lambda_{1}} s_{\left(\lambda_{2}, \lambda_{3}, \ldots, \lambda_{\ell}\right)}^{(k)}=s_{\lambda}^{(k)}+$ other terms which are indexed by $k$ bounded partitions $\gamma$ where $\lambda$ is smaller than $\gamma$ in dominance order.

We begin by noting that $h_{3} s_{311}^{(3)}=s_{3211}^{(3)}$, and we thus must expand $s_{211}^{(3)}$. This time, noting that $h_{2} s_{11}^{(3)}=s_{211}^{(3)}$ we turn to the computation of $s_{11}^{(3)}$. Since $h_{1} s_{1}^{(3)}=s_{11}^{(3)}+s_{2}^{(3)}$ and $s_{2}^{(3)}=h_{2}$, we have computed $s_{11}^{(3)}=h_{11}-h_{2}$ which can be substituted back to obtain $s_{211}^{(3)}=h_{211}-h_{22}$. We conclude that $s_{3211}^{(3)}=h_{3211}-h_{322}$.

We leave it to the reader as an exercise to compute at least a few other of the 3-Schur functions of size 7 by hand to get a feel for the difficulty of these computations. Fortunately, the functions can be verified against a calculation using SAGE.

Sage Example 2.12. Here we give the expansion of the $k$-Schur functions for $k=3$ in terms of the homogeneous basis as we did in the previous example. Our current setting is the $t=1$ case.

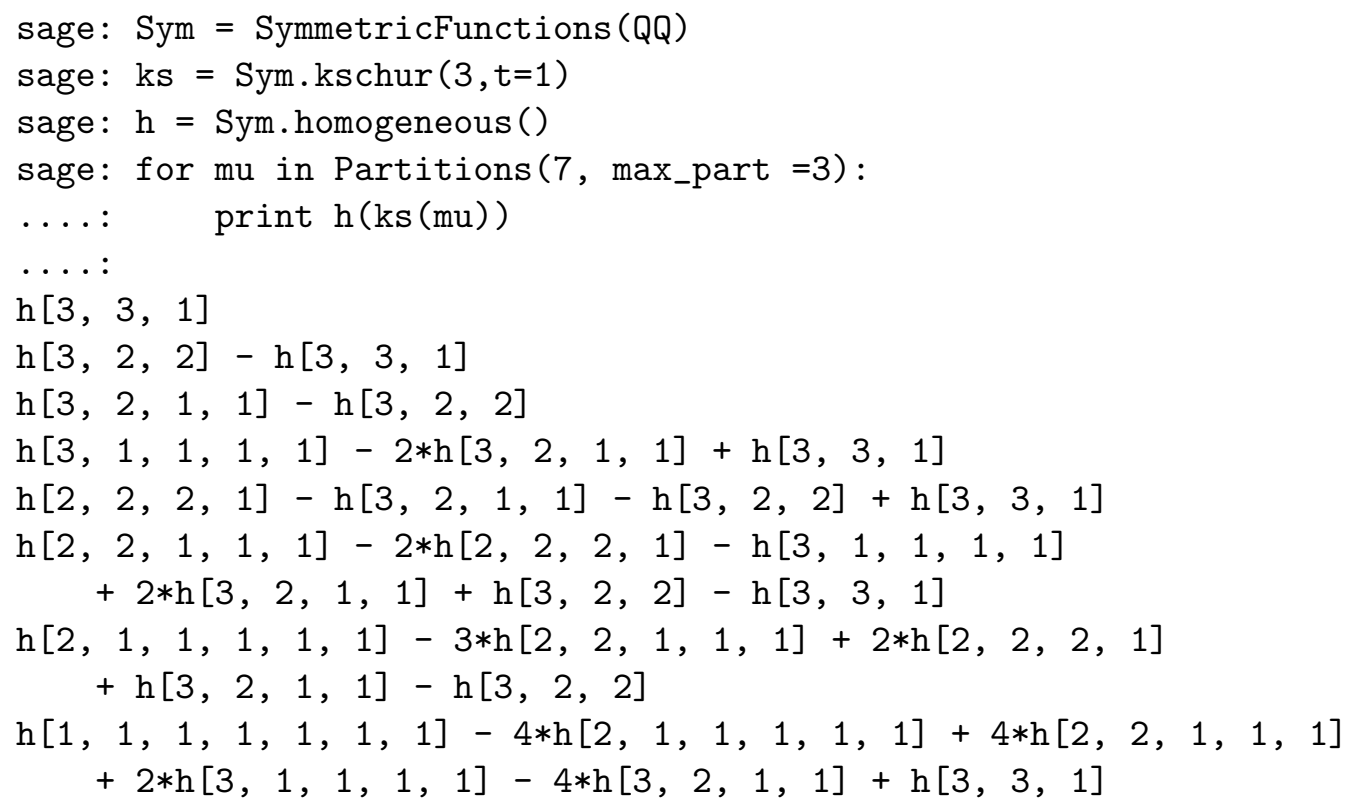

We are now in the position to use duality to produce a second basis, this time for the algebra $\Lambda^{(k)}$. Although we do not have a scalar product on the spaces $\Lambda_{(k)}$ and $\Lambda^{(k)}$ separately, we appeal to a pairing between the two spaces

$$
\langle\cdot, \cdot\rangle: \Lambda_{(k)} \times \Lambda^{(k)} \rightarrow \mathbb{Q}
$$


where $h_{\mu} \in \Lambda_{(k)}$ and $m_{\lambda} \in \Lambda^{(k)}$ are dual elements

$$
\left\langle h_{\mu}, m_{\lambda}\right\rangle=\delta_{\lambda \mu} .
$$

This equation is precisely Equation (1.23) for the scalar product on symmetric functions.

The dual $k$-Schur functions $\tilde{F}_{\lambda}^{(k)}$ were introduced in [97] as the unique basis of the degree $m$ subspace of $\Lambda^{(k)}$ (with $m \geq 1$ ) that is dual to the basis of $\left\{s_{\lambda}^{(k)}\right\}_{\lambda \vdash m, \lambda_{1} \leq k}$ under the pairing (2.15). It follows from (2.14) that the enumeration of weak tableaux gives their monomial expansion:

$$
\begin{aligned}
\tilde{F}_{\lambda}^{(k)} & =\sum_{\mu: \mu_{1} \leq k}\left\langle h_{\mu}, \tilde{F}_{\lambda}^{(k)}\right\rangle m_{\mu} \\
& =\sum_{\mu: \mu_{1} \leq k} \sum_{\gamma: \gamma_{1} \leq k} K_{\gamma \mu}^{(k)}\left\langle s_{\gamma}^{(k)}, \tilde{F}_{\lambda}^{(k)}\right\rangle m_{\mu} \\
& =\sum_{\mu: \mu_{1} \leq k} K_{\lambda \mu}^{(k)} m_{\mu} .
\end{aligned}
$$

There is an involution on the set of weak tableaux of fixed shape $\mathfrak{c}(\lambda)$ and weight $\alpha$ that sends these tableaux to the set of weak tableaux of shape $\mathfrak{c}(\lambda)$ and weight which is a permutation of $\alpha$. Thus, the dual $k$-Schur functions are the weight generating functions for weak tableaux. That is, for $(k+1)$-core $\lambda$,

$$
\tilde{F}_{\lambda}^{(k)}=\sum_{\substack{T=\text { weak tab } \\ \operatorname{shape}(T)=\mathfrak{c}(\lambda)}} x^{\text {weight }(T)} .
$$

Note that what we call the dual $k$-Schur functions here are also equal to affine Stanley symmetric functions indexed by affine Grassmannian elements. This connection is discussed with more detail in Chapter 3, Section 8.2 and Chapter 2, Section 2.5. Note that our notation differs slightly from the notation in Chapter 3 by adding a superscript indicating $k$.

Example 2.13. The calculation of the dual $k$-Schur function $\tilde{F}_{321}^{(3)}$ follows immediately by extracting the weights of each weak tableau of shape $(5,2,1)$, listed in Example 2.8. We conclude that

$$
\tilde{F}_{321}^{(3)}=m_{321}+2 m_{3111}+m_{222}+2 m_{2211}+3 m_{21111}+4 m_{111111} .
$$

\section{$2.3 \quad$ Other realizations}

We have now seen how the weak Pieri rule - given in terms of weak horizontal chains in the $(k+1)$-core realization of the weak poset - leads to the family of dual $k$-Schur functions in terms of weak tableaux. Equivalently, we could have invoked the definition of weak 
horizontal chains on the level of $k$-bounded partitions or on affine Grassmannian elements and this would easily give rise to characterizations for dual $k$-Schur functions in these other settings. Before we move on to draw a weight generating function characterization for $k$ Schur functions starting instead from a "strong" Pieri rule, some exposition on these other interpretations is warranted.

Let us start by retracing our steps that led from the weak Pieri rule to the generating function for dual $k$-Schur functions, this time in the setting of $k$-bounded partitions. In these terms, the weak Pieri relation is given for any partition $\lambda$ with $\lambda_{1} \leq k$ to be

$$
h_{r} s_{\lambda}^{(k)}=\sum_{\mu: \mu_{1} \leq k} s_{\mu}^{(k)}
$$

where the sum is over partitions $\mu$ such that $\mu / \lambda$ is a horizontal strip and $\mu^{\omega_{k}} / \lambda^{\omega_{k}}$ is a vertical strip of size $r$. Again, we use the iteration of this relation to impose conditions on a family of weak tableaux in the $k$-bounded setting.

Example 2.14. Iteratively, we calculate $h_{431}$ in terms of $k$-Schur functions for $k=6$ :

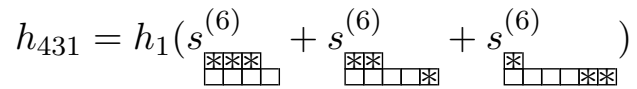

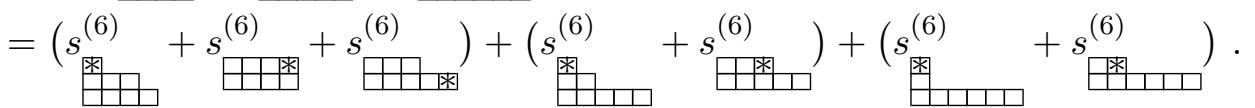

Sage Example 2.15. SAGE can be used to verify Example 2.14:

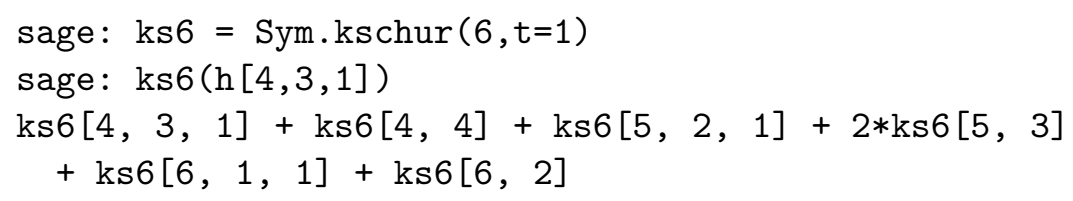

SAGE also knows that the $k$-Schur functions live in the subring $\Lambda_{(k)}$ of the ring of symmetric functions:

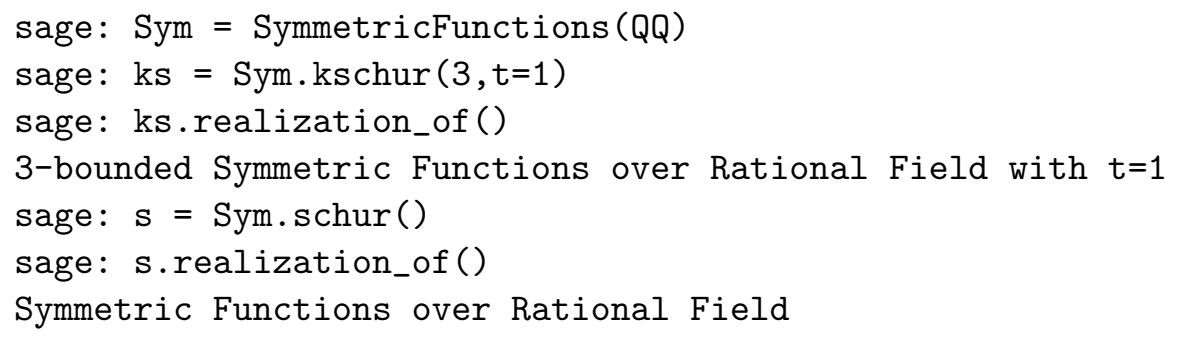

When $\lambda$ and $\mu$ are $k$-bounded partitions, the weak Kostka coefficients $K_{\lambda \mu}^{(k)}$ are interpreted to be the number of sequences of $k$-bounded partitions,

$$
\emptyset=\lambda^{(1)} \subseteq \lambda^{(2)} \subseteq \cdots \subseteq \lambda^{(d)}=\lambda
$$


where $\lambda^{(i)} / \lambda^{(i-1)}$ is a horizontal strip of size $\mu_{i}$ and $\left(\lambda^{(i)}\right)^{\omega_{k}} /\left(\lambda^{(i-1)}\right)^{\omega_{k}}$ is a vertical strip. We then follow the line of reasoning from earlier to yield (2.18), where we can instead think of weak tableaux as these sequences of $k$-bounded shapes.

Example 2.16. The seven tableaux that make up the terms in the expansion of $h_{431}$ in terms of $k$-Schur functions with $k=6$ are

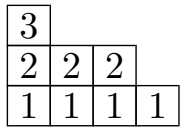

\begin{tabular}{|l|l|l|l|}
\hline 2 & 2 & 2 & 3 \\
\hline 1 & 1 & 1 & 1 \\
\hline
\end{tabular}

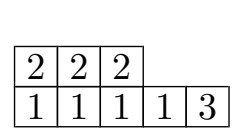

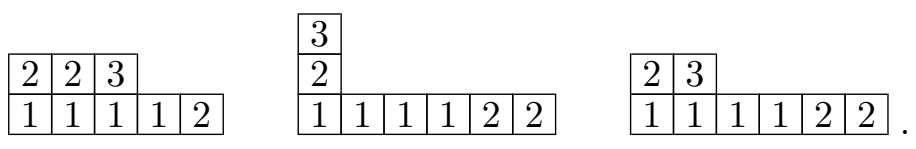

Sage Example 2.17. We can reproduce these tableaux in SAGE using the bounded representation of weak $k$-tableaux:

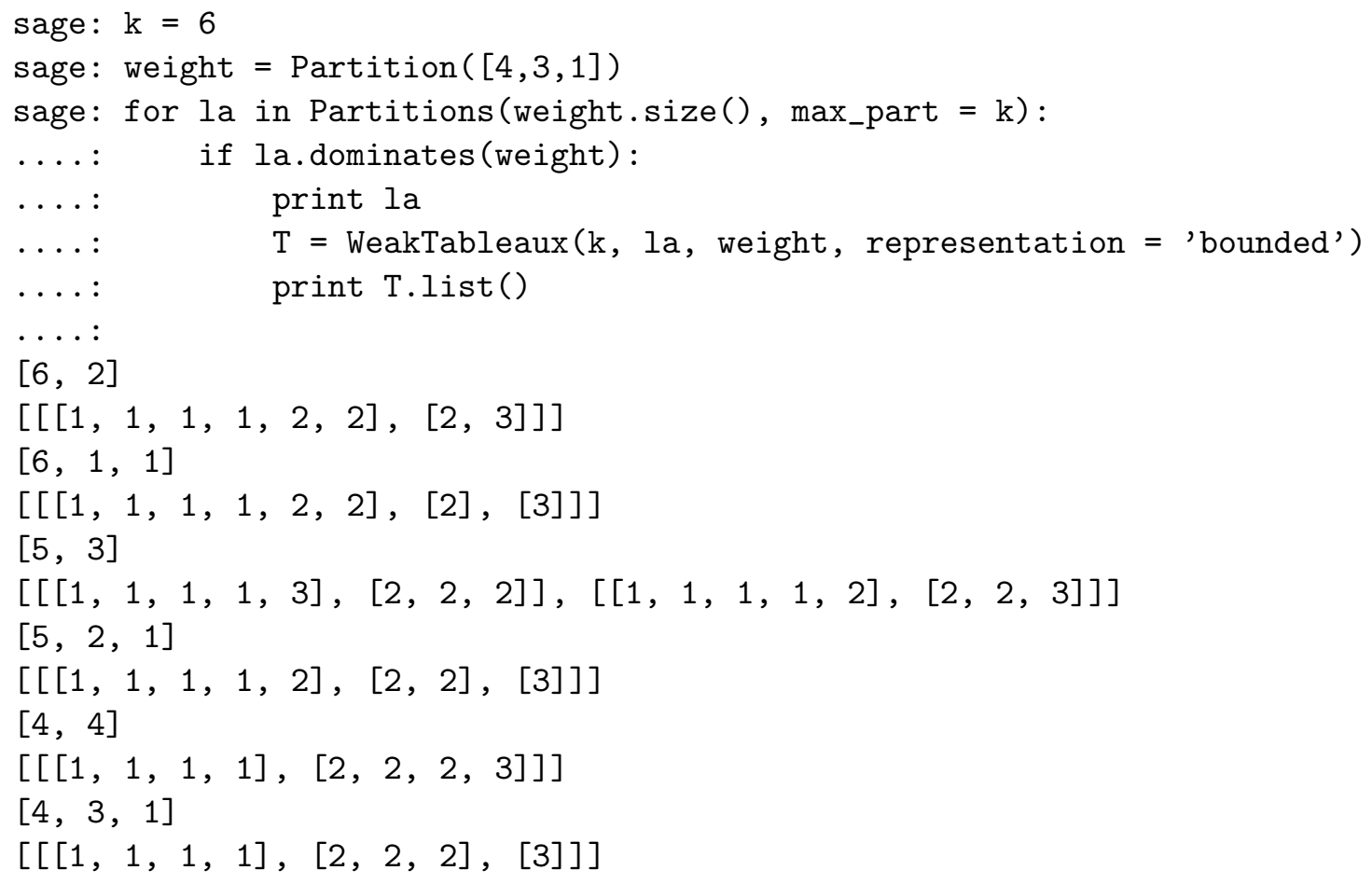

Lastly, let us turn to the language of the affine symmetric group and retrace the steps leading to the dual $k$-Schur functions. Here, the weak Pieri rule is, for a $k$-bounded partition $\lambda$ the corresponding affine Grassmannian element (described in Section 1.2) will be $\mathfrak{a}(\mathfrak{c}(\lambda)$ ) 
which we will shorten to $\mathfrak{a}(\lambda)$. The weak Pieri rule on affine Grassmannian elements can be stated as

$$
h_{r} s_{\lambda}^{(k)}=\sum_{\substack{u=\text { cyclically decreasing } \\ \ell(u)=r, u \mathfrak{a}(\lambda)=\mathfrak{a}(\mu)}} s_{\mu}^{(k)},
$$

where the sum is over cyclically decreasing reduced words such that there is a $k$-bounded partition $\mu$ such that $u \mathfrak{a}(\lambda)=\mathfrak{a}(\mu)$.

The iteration of this relation produces another interpretation of the weak Kostka numbers. First, for any $k$-bounded partition $\mu$, define a $\mu$-factorization of $w$ to be a decomposition of the form $w=w^{\ell(\mu)} \cdots w^{1}$ where each $w^{i}$ is a cyclically decreasing element of length $\mu_{i}$. Then, for $k$-bounded partitions $\lambda$ and $\mu, K_{\lambda, \mu}^{(k)}$ is the number of $\mu$-factorizations of $w=\mathfrak{a}(\lambda)$. In particular, if we consider the case that $\mu=(1,1, \ldots, 1)$, then the weak Kostka number $K_{\lambda,\left(1^{n}\right)}^{(k)}$ is precisely the number of reduced words for $w=\mathfrak{a}(\lambda)$.

Example 2.18. Note from the previous example that the coefficient of $m_{111111}$ in $\tilde{F}_{521}^{(k)}$ is 4 indicating that there are 4 reduced words in $\tilde{S}_{3}$ for the affine Grassmannian element corresponding to the core $(5,2,1)$; they are $s_{2} s_{0} s_{3} s_{2} s_{1} s_{0}=s_{0} s_{2} s_{3} s_{2} s_{1} s_{0}=s_{0} s_{3} s_{2} s_{3} s_{1} s_{0}=$ $s_{0} s_{3} s_{2} s_{1} s_{3} s_{0}$.

From here, we again use the line of reasoning and duality to arrive at the interpretation for dual $k$-Schur functions as

$$
\tilde{F}_{w}^{(k)}=\sum_{\mu} K_{\mathfrak{a}^{-1}(w) \mu}^{(k)} m_{\mu}
$$

where we have indexed the dual $k$-Schur function by an affine Grassmannian permutation to emphasize that the coefficients $K_{\mathfrak{a}^{-1}(w) \mu}^{(k)}$ represent $\mu$-factorizations of $w$. From the definition and symmetry of the weak Kostka numbers, the dual $k$-Schur functions can be suggestively written as the weight generating function:

$$
\tilde{F}_{w}^{(k)}=\sum_{w=w^{1} w^{2} \ldots w^{r}} x^{\ell\left(w^{1}\right)} x^{\ell\left(w^{2}\right)} \cdots x^{\ell\left(w^{r}\right)},
$$

over all factorizations of $w$ into products of cyclically decreasing $w^{i}$.

This interpretation for dual $k$-Schur functions is the starting point in [78] to a family of symmetric functions called affine Stanley symmetric functions where the condition that $w$ is affine Grassmannian is relaxed and we allow the function to be indexed by arbitrary affine permutations (not just an affine Grassmannian element). For more information see Chapter 3, Definition 3.2.

Programs to compute affine Stanley functions are in SAGE and we can do computations with dual $k$-Schur functions by taking affine Grassmannian elements.

Sage Example 2.19. We verify Example 2.13 in SAGE by first converting the indexing partition $(3,2,1)$ to an affine Grassmannian element: 


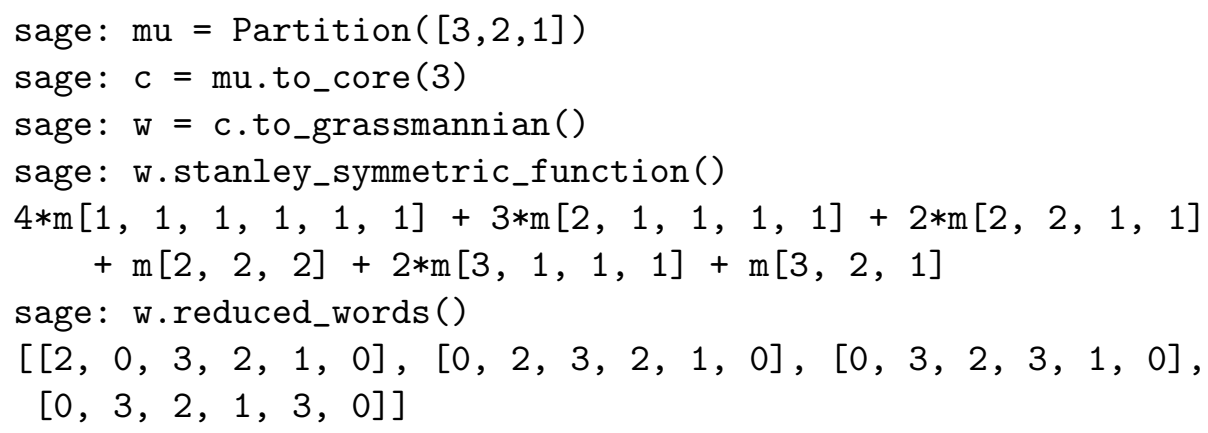

Alternatively, we can access the dual $k$-Schur functions from the quotient space:

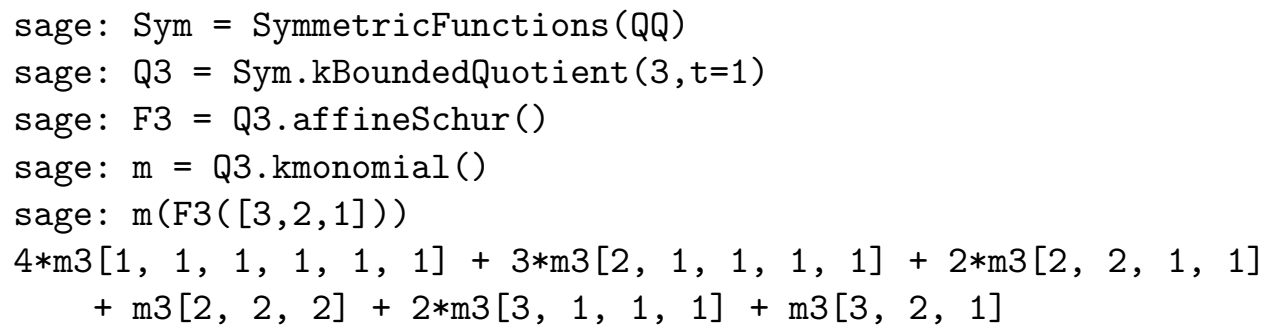

\subsection{Strong marked tableaux and a monomial expansion of $k$-Schur func- tions}

The Pieri rule for the dual $k$-Schur functions $\tilde{F}_{\lambda}^{(k)}$ is probably less intuitive than the one for the $k$-Schur functions because it does have coefficients in the expansion which are not simply 1 or 0 . In the last section we gave an explicit definition of the dual $k$-Schur functions in (2.17), so it is possible to experiment with these elements to see how they behave under multiplication by an element $h_{r} \in \Lambda^{(k)}$. Through the following computations we hope to demonstrate how it might be possible to experiment with data for the Pieri rule for the dual $k$-Schur functions and then later explain what that Pieri rule is.

Example 2.20. In Example 2.13 we computed the dual $k$-Schur function indexed by $(3,2,1)$ for $k=3$. Using the tableau definition of the dual $k$-Schur functions from Equation (2.17) it is possible to expand $\tilde{F}_{\lambda}^{(3)}$ for all 3-bounded partitions $\lambda$ of size 7 and use this to find the expansion of $h_{1} \tilde{F}_{321}^{(3)}$. If the $k$-Schur functions up to size 7 are already known, then this also can be computed using the duality. In particular we obtain

$$
h_{1} \tilde{F}_{321}^{(3)}=2 \tilde{F}_{331}^{(3)}+\tilde{F}_{322}^{(3)}+3 \tilde{F}_{3211}^{(3)}+\tilde{F}_{31111}^{(3)} .
$$

This example demonstrates that if a dual $k$-Schur function indexed by a partition $\lambda$ is multiplied by $h_{1}$, the resulting partitions indexing the functions in the expansion do not necessarily contain $\lambda$ (notice that $(3,2,1)$ is not contained in $(3,1,1,1,1)$ ). 
Sage Example 2.21. We now demonstrate how the computations for Example 2.20 can be carried out in SAGE. The dual $k$-Schur functions can be accessed through the $k$-bounded quotient space:

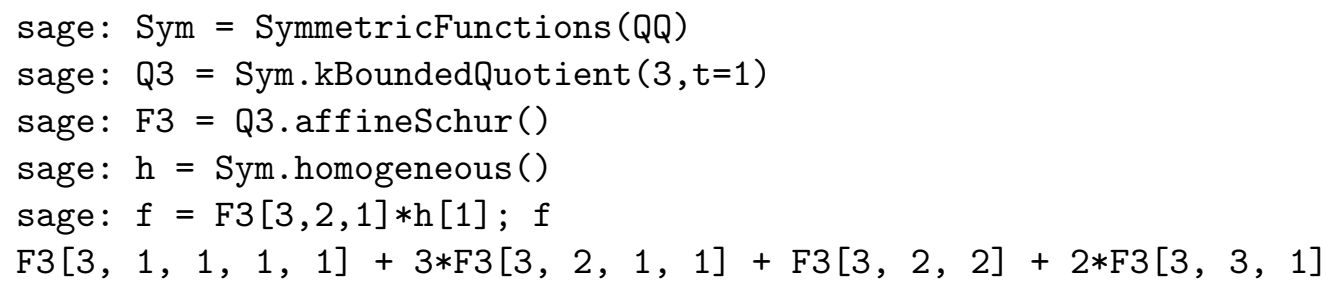

In terms of 3-bounded partitions, it is not so clear how to interpret the coefficients in the expansion in Example 2.20. However, in terms of 4-cores it becomes more obvious. The 4-core of $(3,2,1)$ is contained in the cores of the partitions in the expansion:

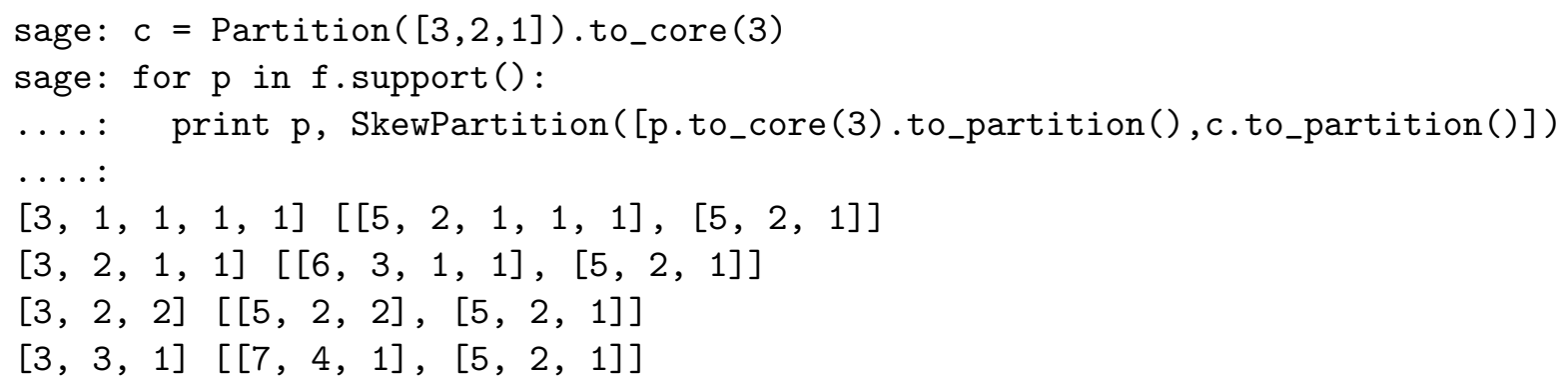

The corresponding skew diagrams are
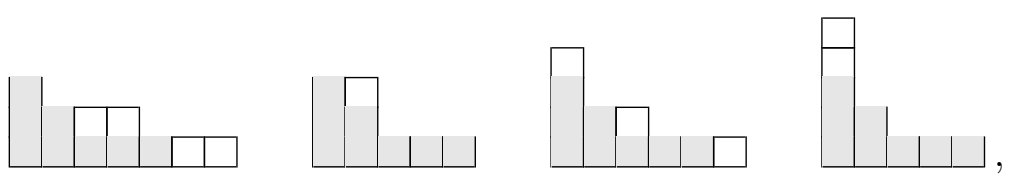

respectively. Comparing with (2.24), one is led to conjecture that the coefficient of $\tilde{F}_{\mu}^{(k)}$ in the expansion of $h_{1} \tilde{F}_{\lambda}^{(k)}$ equals the number of connected components of $\mathfrak{c}(\mu) / \mathfrak{c}(\lambda)$. Alternatively, we may mark the lowest rightmost cell of one of these connected components and the number of ways of marking is equal to this coefficient.

Recall that we defined a strong marked horizontal strip of size $r$ in Equation (1.10) as a sequence cores

$$
\kappa^{(0)} \Rightarrow_{k} \kappa^{(1)} \Rightarrow_{k} \kappa^{(2)} \Rightarrow_{k} \cdots \Rightarrow_{k} \kappa^{(r)}
$$

and integers $c_{1}<c_{2}<\cdots<c_{r}$ such that $\left(\kappa^{(i)}, \kappa^{(i-1)}, c_{i}\right)$ is a marking for each $1 \leq i \leq r$. It is in these terms that we state the Pieri rule for dual $k$-Schur functions. 
Theorem 2.22. [81, Theorem 4.9] For $r \geq 1$,

$$
h_{r} \tilde{F}_{\lambda}^{(k)}=\sum_{\left(\kappa^{(*)}, c_{*}\right)} \tilde{F}_{\mathfrak{p}\left(\kappa^{(r)}\right)}^{(k)},
$$

where the sum is over all strong marked horizontal strips

$$
\kappa^{(*)}=\left(\mathfrak{c}(\lambda)=\kappa^{(0)} \Rightarrow_{k} \kappa^{(1)} \Rightarrow_{k} \kappa^{(2)} \Rightarrow_{k} \cdots \Rightarrow_{k} \kappa^{(r)}\right)
$$

with markings $c_{*}=\left(c_{1}<c_{2}<\cdots<c_{r}\right)$.

Iterating the Pieri rule on the dual $k$-Schur functions defines a different notion of 'semistandard tableaux'. We say that a strong marked tableau of shape $\lambda \vdash m$ (or shape $\mathfrak{c}(\lambda)$ if the shape is more properly given as a $(k+1)$-core $)$ and content $\alpha=\left(\alpha_{1}, \alpha_{2}, \ldots, \alpha_{d}\right)$ with $\alpha_{1}+\alpha_{2}+\cdots+\alpha_{d}=m$ and $\alpha_{i} \geq 1$ is a sequence of $(k+1)$-cores

$$
\kappa^{(0)}=\emptyset \Rightarrow_{k} \kappa^{(1)} \Rightarrow_{k} \kappa^{(2)} \Rightarrow_{k} \cdots \Rightarrow_{k} \kappa^{(m)}=\mathfrak{c}(\lambda)
$$

and markings $c_{*}=\left(c_{1}, c_{2}, \ldots, c_{m}\right)$ such that $\left(\kappa^{(v)}, \kappa^{(v+1)}, \ldots, \kappa^{\left(v+\alpha_{r}\right)}\right)$ and $\left(c_{v+1}, c_{v+2}, \ldots, c_{v+\alpha_{r}}\right)$ with $v=\alpha_{1}+\cdots+\alpha_{r-1}$ forms a strong marked horizontal strip for each $1 \leq r \leq d$.

Recall from Section 1.4 that a strong marked cover is also an application of a transposition $t_{i j}$ in the affine symmetric group to a core (either by the left or right action, see Proposition 1.27). Therefore, it is possible to also view a tableau as an element of the affine Grassmannian written as a sequence of these transpositions. The condition that a sequence of transpositions $t_{i_{a+b} j_{a+b}} \cdots t_{i_{a+2} j_{a+2}} t_{i_{a+1} j_{a+1}} t_{i_{a} j_{a}}$ forms a strong marked horizontal strip (with the left action) implies that $j_{a}<j_{a+1}<\cdots<j_{a+b}$.

Example 2.23. Consider the path

$$
\begin{aligned}
\emptyset \Rightarrow_{3}(1) \Rightarrow_{3}(2) \Rightarrow_{3}(2,1) \Rightarrow_{3}(3,1,1) \Rightarrow_{3}(3,1,1,1) \Rightarrow_{3}(3,3,1,1) & \\
& \Rightarrow_{3}(4,3,2,1) \Rightarrow_{3}(4,4,2,2) .
\end{aligned}
$$

We choose $\alpha=(2,2,3,1)$ and a sequence $c=(0,1,-1,2,-3,1,3,-2)$ as a sequence of markings which form strong marked horizontal strips of the lengths given by the entries of $\alpha$ to demonstrate the concept of a strong marked tableau. The strong marked tableau of shape $(4,4,2,2)$ records all cells representing strong marked horizontal strips labeled with the same main label but subscripted by which set of ribbons they belong to. The cell which is marked (the head of one of the ribbons) will be indicated by including an $*$ as a superscript. Hence the example above is represented by the diagram:

$$
\begin{aligned}
& \begin{array}{|l|l|}
\hline 3_{1}^{*} & 4_{1}^{*} \\
\hline 2 & 3 \\
\hline
\end{array} \\
& \begin{array}{lll}
2_{2} & 3_{3} \\
\hline
\end{array} \\
& \begin{array}{|l|l|l|l|}
\hline 2_{1}^{*} & 3_{2} & 3_{2}^{*} & 4_{1} \\
\hline
\end{array} \\
& \begin{array}{|l|l|l|l|}
\hline 1_{1}^{*} & 1_{2}^{*} & 2_{2}^{*} & 3_{3}^{*} \\
\hline
\end{array}
\end{aligned}
$$


This example is also represented by the following sequence of transpositions with the left action

$$
t_{-2-1} t_{34} t_{02} t_{-3-2} t_{23} t_{-10} t_{12} t_{01}
$$

which act on the empty 4-core. Each transposition adds the strong marking in a horizontal strip according to the tableau.

This same example is also represented using the right action of the transpositions by

$$
t_{01} t_{02} t_{-11} t_{05} t_{-21} t_{-12} t_{06} t_{-52}
$$

Just as we did for semi-standard and weak tableaux, we set $\mathbf{K}_{\lambda \mu}^{(k)}$ to be the number of strong marked tableaux of shape $\lambda$ and weight $\mu$. By iterating the Pieri rule on dual $k$ Schur functions and using the notion of strong marked tableaux to record the terms which appear in the expansion of products of elements of the homogeneous symmetric functions, we have that for a partition $\mu \vdash m$ (not necessarily $k$-bounded)

$$
h_{\mu}=\sum_{\lambda: \lambda_{1} \leq k} \mathbf{K}_{\lambda \mu}^{(k)} \tilde{F}_{\lambda}^{(k)}
$$

Then for a partition $\lambda \vdash m$ with $\lambda_{1} \leq k$,

$$
s_{\lambda}^{(k)}=\sum_{\mu}\left\langle s_{\lambda}^{(k)}, h_{\mu}\right\rangle m_{\mu}=\sum_{\mu} \mathbf{K}_{\lambda \mu}^{(k)} m_{\mu}
$$

We have hidden all of the work that is needed to show the monomial expansions of $k$-Schur functions and their duals are correct by this presentation. In fact, the proof [81] follows a nearly reverse path of reasoning to demonstrate the results we have presented here. Equation (2.29) is taken as the definition of the $k$-Schur functions and (2.17) is the definition of the dual $k$-Schur functions, and then the argument uses combinatorics and algebraic expressions to show that these elements are dual. The combinatorial part of that argument is developed in Section 6.

Example 2.24. Consider the coefficient of $m_{421}$ in $s_{3211}^{(3)}$. It is calculated by finding all strong marked tableaux that begin with a horizontal strip of length 4 and hence contains

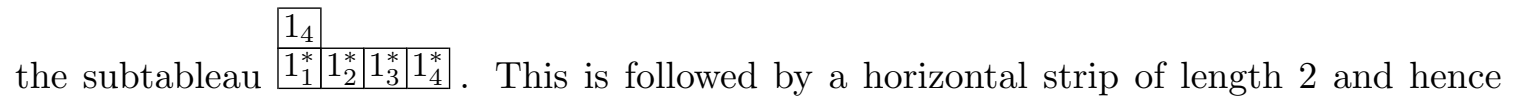
contains one of the five subtableaux:

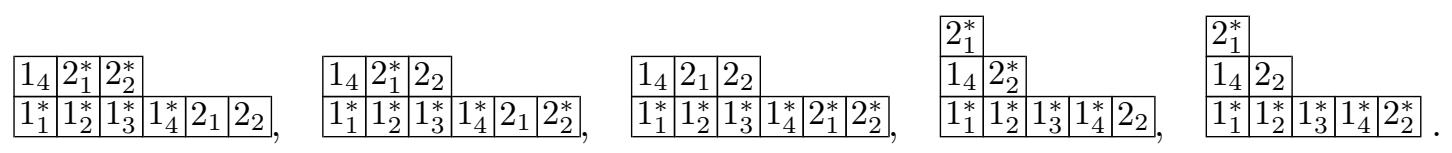


There are 9 strong marked tableaux of shape $\mathfrak{c}(3,2,1,1)=(6,3,1,1)$ of weight $(4,2,1)$ which are given by the following
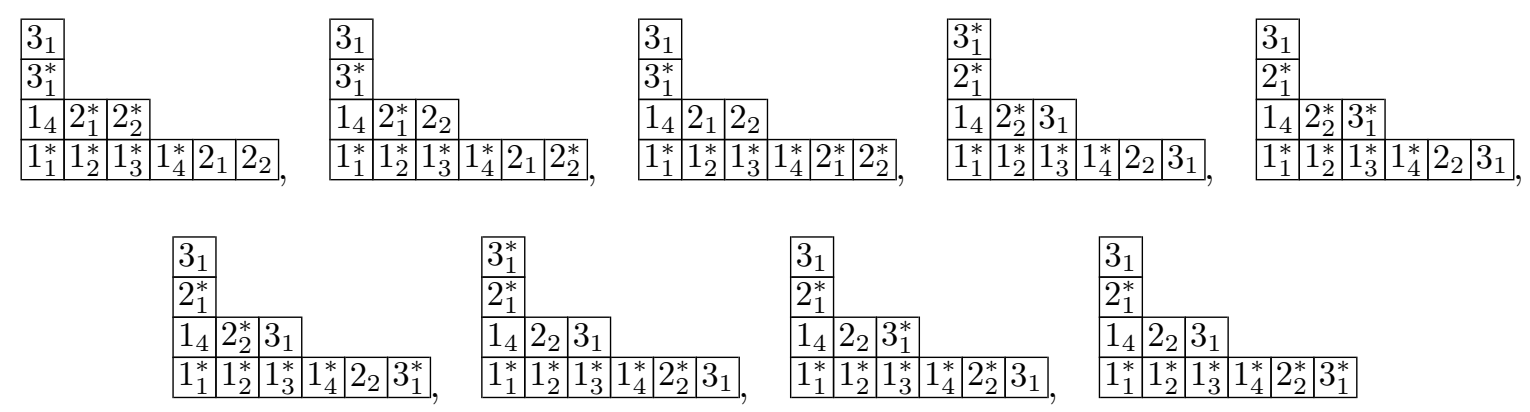

A similar argument can be used to find any coefficient of $m_{\mu}$ for any $\mu \vdash 7$, however there are 210 strong marked tableaux of shape $\mathfrak{c}(3,2,1,1)=(6,3,1,1)$ and weight $(1,1,1,1,1,1,1)$ and hence we will not show the complete computation of $s_{3211}^{(3)}$ using this method.

In later sections we will roughly outline how this formula is proven by generalizing the Robinson-Schensted-Knuth algorithm.

Sage Example 2.25. In this example we show how SAGE can be used to complete calculations from Examples 2.23 and 2.24. The strong tableaux can be entered as a list of entries with markings indicated by a negative number.

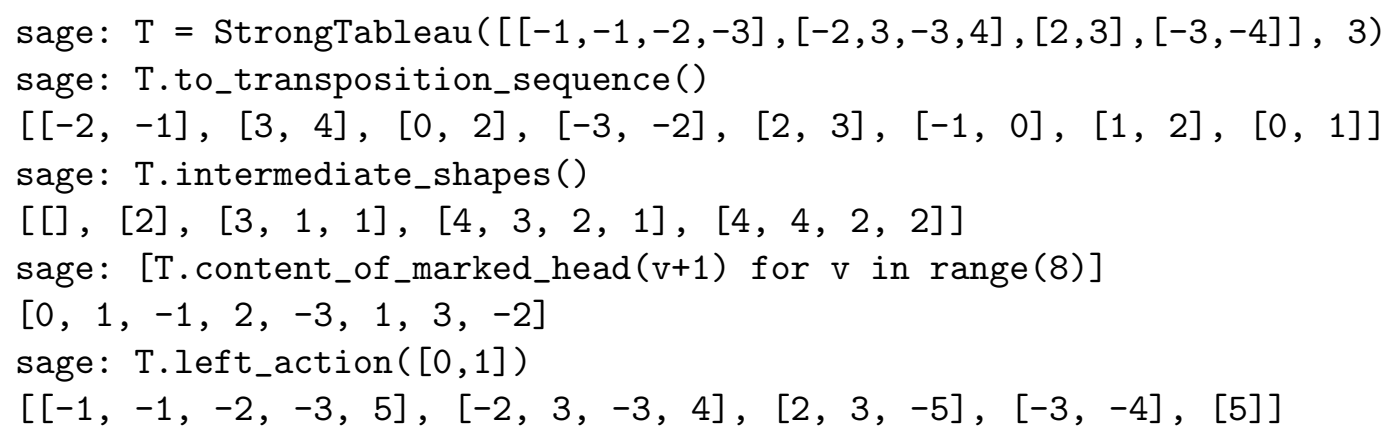

The strong tableaux can be listed and by equation (2.29) the number of strong tableaux of shape $\mathfrak{c}(\lambda)$ and content $\mu$ can be calculated by determining the coefficient of $m_{\mu}$ in $s_{\lambda}^{(k)}$.

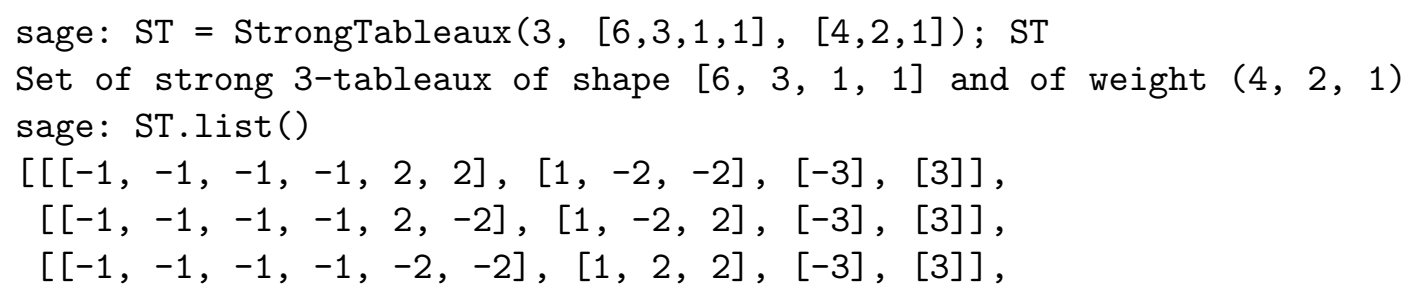




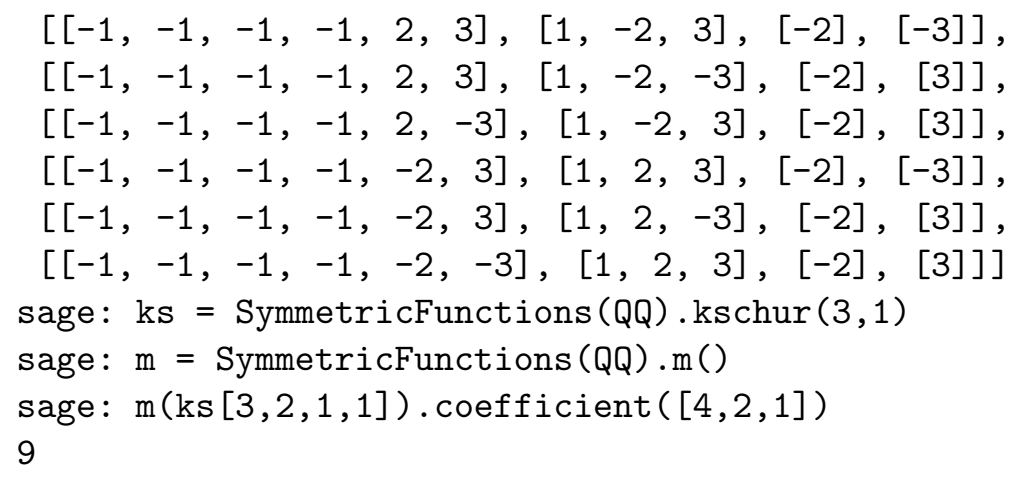

Remark 2.26. We have been purposely lax in our notation to make some of the concepts slightly easier to follow, but the duality creates a few issues with the element which represents $\tilde{F}_{\lambda}^{(k)}$ in $\Lambda^{(k)}$. The equalities in Equations (2.25) and (2.28) represent equality in the realization of the dual algebra $\Lambda^{(k)}=\Lambda /\left\langle m_{\lambda}: \lambda_{1}>k\right\rangle$, so the equality really means equivalence in the quotient algebra. For computational purposes, we would typically take a representative element from the linear span of $\left\{m_{\lambda}\right\}_{\lambda_{1} \leq k}$, but for certain purposes a multiplicative basis might be more desirable. In those cases, the basis $\left\{p_{\lambda}\right\}_{\lambda_{1} \leq k}$ works well since $p_{r} \in\left\langle m_{\lambda}: \lambda_{1}>k\right\rangle$ for $r>k$. A combinatorial formula for $\tilde{F}_{\lambda}^{(k)}$ in terms of the power sum basis is also known by reference [7].

\section{$2.5 \quad k$-Littlewood-Richardson coefficients}

Although the original definition [92] of $k$-Schur functions was inspired to explain the positivity of the expansion of Macdonald symmetric functions in terms of Schur functions, it has since been established that the theory of $k$-Schur functions and their duals can be naturally applied to study problems in geometry, physics and representation theory.

The application of $k$-Schur functions to geometric problems began when Lapointe and Morse discovered that their structure constants could be identified with certain geometric invariants in a way that mimics the identification of Littlewood-Richardson coefficients with Schubert structure constants in the Grassmannian variety (recall from Section 1.6); computation in the quantum cohomology of Grassmannians reduces to $k$-Schur calculations. The (small) quantum cohomology ring $Q H^{*}\left(\mathrm{Gr}_{\ell n}\right)$ is a deformation of the classical cohomology ring that is motivated by ideas in string theory (e.g. $[1,156]$ ). As abelian groups, $Q H^{*}\left(\mathrm{Gr}_{\ell n}\right)=H^{*}\left(\mathrm{Gr}_{\ell n}\right) \otimes \mathbb{Z}[q]$ and the Schubert classes $\sigma_{\lambda}$ with $\lambda \in \mathcal{P}^{\ell n}$ form a $\mathbb{Z}[q]$-linear basis, where recall that $\mathcal{P}^{\ell n}$ is the set of all partitions in an $\ell \times(n-\ell)$ rectangle. The appeal lies in the multiplicative structure which is defined by

$$
\sigma_{\lambda} * \sigma_{\mu}=\sum_{\substack{\nu \in \mathcal{P} \ell n \\|\nu|=|\lambda|+|\mu|-d n}} q^{d} C_{\lambda \mu}^{\nu, d} \sigma_{\nu},
$$


where $C_{\lambda \mu}^{\nu, d}$ are the 3-point Gromov-Witten invariants, counting the number of rational curves of degree $d$ in $\mathrm{Gr}_{\ell n}$ that meet generic translates of certain Schubert varieties.

As with the usual cohomology, the quantum cohomology ring can be connected to symmetric functions. In particular,

$$
Q H^{*}\left(\operatorname{Gr}_{\ell n}\right) \cong\left(\Lambda_{(\ell)} \otimes \mathbb{Z}[q]\right) / J_{q}^{\ell n}
$$

where $J_{q}^{\ell n}=\left\langle e_{n-\ell+1}, \ldots, e_{n-1}, e_{n}+(-1)^{\ell} q\right\rangle$. When $\lambda \in \mathcal{P}^{\ell n}$, the Schubert class $\sigma_{\lambda}$ still maps to the Schur function $s_{\lambda}$ implying that for $\lambda, \mu \in \mathcal{P}^{\ell n}$,

$$
\sum_{\nu} c_{\lambda \mu}^{\nu} s_{\nu} \bmod J_{q}^{\ell n}=\sum_{\substack{\nu \in \mathcal{P} \ell n \\|\nu|=|\lambda|+|\mu|-d n}} q^{d} C_{\lambda \mu}^{\nu, d} s_{\nu}
$$

Unfortunately, there are $\nu \notin \mathcal{P}^{\ell n}$ where $s_{\nu}$ does not lie in $J_{q}^{\ell n}$. Instead, reduction modulo this ideal requires a complicated algorithm [16, 34, 67, 157] involving negatives. Therefore, the Schur functions cannot be used to directly obtain the quantum structure constants.

It was proven in [97] that the $k$-Schur basis can be used to circumvent this problem; the appropriate $k$-Schur functions lie in $J_{q}^{\ell n}$ (as usual, we set $n=k+1$ ). To be precise, the $k$-Littlewood-Richardson coefficients are the structure coefficients $c_{\lambda \mu}^{\nu(k)}$ of the algebra of $k$-Schur functions

$$
s_{\lambda}^{(k)} s_{\mu}^{(k)}=\sum_{\nu} c_{\lambda \mu}^{\nu(k)} s_{\nu}^{(k)}
$$

Let $\Pi^{\ell, k+1}$ be the set of partitions with no part larger than $\ell$ and no more than $k+1-\ell$ rows of length smaller than $\ell$. It is proven that $s_{\nu}^{(k)}$ modulo $J_{q}^{\ell n}$ reduces to a power of $q$ times a positive $s_{\mathfrak{r}(\nu)}$ when $\nu \in \Pi^{\ell n}$ and is otherwise zero (where $\mathfrak{r}(\nu) \in \mathcal{P}^{\ell n}$ is the $n$-core of $\nu$ ). Thus, considering

$$
s_{\lambda}^{(k)} s_{\mu}^{(k)}=\sum_{\nu \in \Pi^{\ell, k+1}} a_{\lambda \mu}^{\nu(k)} s_{\nu}^{(k)}+\sum_{\nu \notin \Pi^{\ell, k+1}} c_{\lambda \mu}^{\nu, k} s_{\nu}^{(k)},
$$

the 3-point Gromov-Witten invariants are none other than a special case of $k$-LittlewoodRichardson coefficients; for $\lambda, \mu, \nu \in \mathcal{P}^{\ell n}$,

$$
C_{\lambda \mu}^{\nu, d}=a_{\lambda \mu}^{\hat{\nu}(n-1)}
$$

where the value of $d$ associates a certain unique element $\hat{\nu} \in \Pi^{\ell n}$ to each $\nu$. It also follows that the $k$-Littlewood-Richardson coefficients include the fusion rules for the WessZumino-Witten conformal field theories associated to $\widehat{s u}(\ell)$ at level $k+1-\ell$ and certain Hecke algebra structure constants studied by Goodman and Wenzl in [56].

Note that the quantum structure constants (2.30) are only a subset of the complete set of $k$-Littlewood-Richardson coefficients (2.32). To understand the bigger picture, recall that 
the $k$-Schur functions are a basis for $\Lambda_{(k)}$ and that $\Lambda_{(k)} \cong H_{*}(\mathrm{Gr})$ where Gr is the affine Grassmannian quotient $\mathrm{Gr}=S L_{k+1}(\mathbb{C}((t))) / S L_{k+1}(\mathbb{C}[[t]])[22]$. Bott also showed that $H^{*}(\mathrm{Gr}) \cong \Lambda^{(k)}$, the space equipped with the dual $k$-Schur basis. Morse and Shimozono conjectured that the $k$-Schur functions and their duals are isomorphic to the Schubert classes of the homology and cohomology of the affine Grassmannian. Chapter 3 explains how Lam proved this conjecture.

Many attempts have been made to understand the coefficients $c_{\lambda \mu}^{\nu(k)}$ but the complete combinatorial picture has yet to be drawn. Knutson formulated a conjecture for the subset of quantum Littlewood-Richardson coefficients as presented in [29] in terms of puzzles [74]. Coskun [33] gave a positive geometric rule to compute the structure constants of the cohomology ring of two-step flag varieties in terms of Mondrian tableaux. As will be discussed in Section 4.6, the case when either $\lambda$ or $\mu$ is a rectangle with a hook of size $k$ is special. Lapointe and Morse [96] showed that if $R$ is a rectangular partition with maximal hook $k$, then

$$
s_{R}^{(k)} s_{\lambda}^{(k)}=s_{R \cup \lambda}^{(k)} .
$$

In $[125,126]$, Morse and Schilling define crystal operators on $\alpha$-factorizations (or equivalently weak $k$-tableaux) to determine some structure coefficients of the Schur function times $k$-Schur function expansion using a sign-reversing involution. This includes the case of fusion coefficients.

Recently there has been some progress in the understanding of these coefficients by viewing them in terms of the nil-Coxeter algebra. Slightly change the setting from the affine symmetric group to the affine nil-Coxeter algebra $\mathbb{A}_{n}$ of type $A_{n-1}$ defined by the generators $A_{0}, A_{1}, A_{2}, \ldots, A_{n-1}$ satisfying the same relations as the affine symmetric group Equation (1.4) except that the quadratic relation is altered to be

$$
A_{i}^{2}=0,
$$

for any $i \in I:=\{0,1,2, \ldots, n-1\}$.

As before, $n=k+1$. For $1 \leq r \leq k$, we set

$$
\tilde{\mathbf{h}}_{r}=\sum_{\substack{w \text { cyclically decreasing } \\ \ell(w)=r}} A_{w} .
$$

Note that the coefficient of $x^{\alpha}$ in (2.23) is the coefficient of $A_{w}$ in $\tilde{\mathbf{h}}_{\alpha_{1}} \tilde{\mathbf{h}}_{\alpha_{2}} \cdots \tilde{\mathbf{h}}_{\alpha_{\ell}}$ and thus, the affine Stanley symmetric function is

$$
\tilde{F}_{w}=\sum_{\alpha}\left(\text { coefficient of } A_{w} \in \tilde{\mathbf{h}}_{\alpha_{1}} \tilde{\mathbf{h}}_{\alpha_{2}} \cdots \tilde{\mathbf{h}}_{\alpha_{\ell}}\right) x^{\alpha} .
$$

As is stated in Theorem 8.6 of Chapter 3, the elements $\tilde{\mathbf{h}}_{r}$ mutually commute and there is a subalgebra $\mathbb{B}_{\mathrm{af}} \subseteq \mathbb{A}_{n}$ which is generated by the elements $\tilde{\mathbf{h}}_{r}$ for $1 \leq r \leq k$. 
Moreover, $\mathbb{B}_{\mathrm{af}}$ is isomorphic to $\Lambda_{(k)}$ (see Proposition 8.8 of Chapter 3) and there is a coalgebra structure which encodes the structure of the dual algebra. The noncommutative $k$-Schur functions can be realized as elements $\mathbf{s}_{\lambda}^{(k)} \in \mathbb{B}_{\text {af }}$ in this algebra by expanding $s_{\lambda}^{(k)}$ in the homogeneous generators $h_{i}$ and replacing $h_{i}$ with $\tilde{\mathbf{h}}_{i}$.

Let $a_{\lambda w}$ be the coefficients in the expansion of $\mathbf{s}_{\lambda}^{(k)}$ in terms of expressions in the generators $A_{i}$ in the affine nil-Coxeter algebra

$$
\mathbf{s}_{\lambda}^{(k)}=\sum_{w} a_{\lambda w} A_{w}
$$

The expansion of $\mathbf{s}_{\lambda}^{(k)}$ has a single term indexed by an affine Grassmannian permutation, it is precisely the affine Grassmannian permutation which corresponds to the partition $\lambda$ and the coefficient $a_{\lambda w_{\lambda}}=1$ where $\mathfrak{a}(\lambda)=w_{\lambda}$.

By [78, Proposition 6.7], the coefficient $a_{\lambda w}$ is the coefficient of $\tilde{F}_{\lambda}$ in the element $\tilde{F}_{w}$. In [79], these coefficients were shown to be positive. Furthermore, by the arguments in [78, Section 17] it follows that

$$
c_{\lambda \mu}^{\nu(k)}=a_{\lambda v w^{-1}}
$$

where $w=\mathfrak{a}(\mu)$ and $v=\mathfrak{a}(\nu)$, see Proposition 1.9.

Berg, Bergeron, Thomas, and Zabrocki [11] gave a combinatorial expansion of $k$-Schur functions indexed by a rectangle with maximal hook $k$ in the nil-Coxeter algebra of the form of Equation (2.34). This work was extended by Berg, Saliola, and Serrano [14] to give expansions of $k$-Schur functions when the indexing partition is a 'maximal rectangle' with a smaller hook removed. In addition they proved some conjectures of [81] in [13], in particular that "skew shaped" strong Schur functions are symmetric.

Sage Example 2.27. We now show how to compute the noncommutative Schur functions in the affine nil-Coxeter algebra $\mathbb{A}_{n}$. We can construct all cyclically decreasing words from the reduced words of the Pieri factors for the affine type $A_{n-1}$ where $n=k+1$.

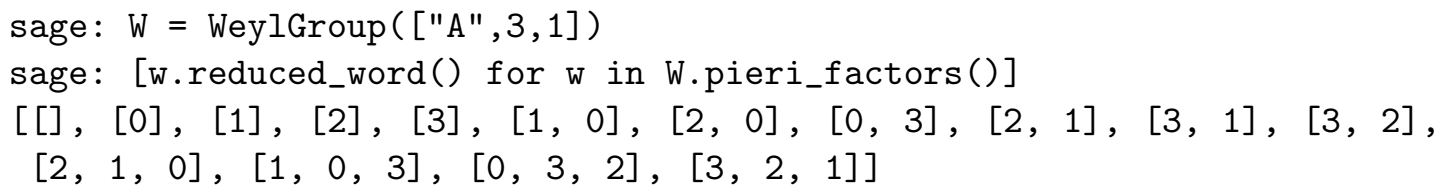

Then the noncommutative homogeneous symmetric functions are given by summing over all cyclically decreasing words of specified length:

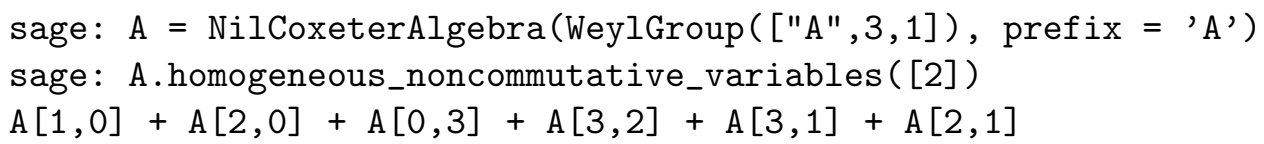

The noncommutative $k$-Schur functions are obtained by expanding the usual $k$-Schur functions in terms of the homogeneous symmetric function: 


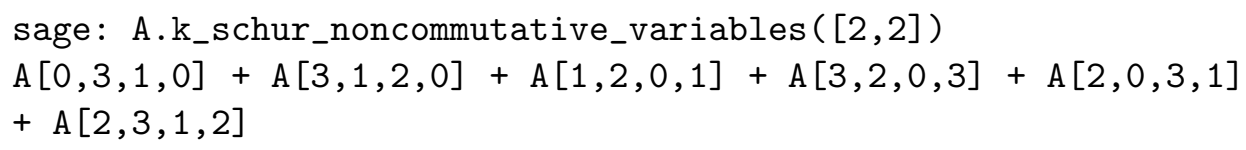

Now let us test that $a_{\lambda w}$ is indeed related to $c_{\lambda \mu}^{\nu(k)}$. The structure coefficient $c_{21,21}^{321(5)}=2$ as we can see from the following computation:

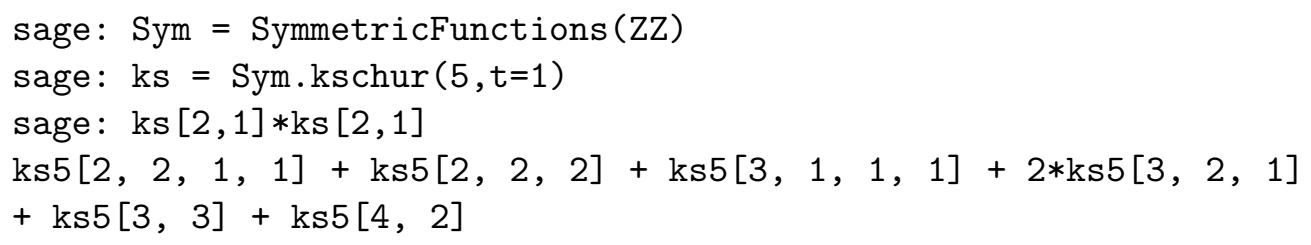

Let $v$ (resp. $w$ ) be the affine Grassmannian element corresponding to the 5-bounded partition $(3,2,1)$ (resp. $(2,1)$ ). Then by $(2.35)$ the coefficient of $A_{v w^{-1}}$ in $\mathbf{s}_{21}^{(5)}$ should also be 2 :

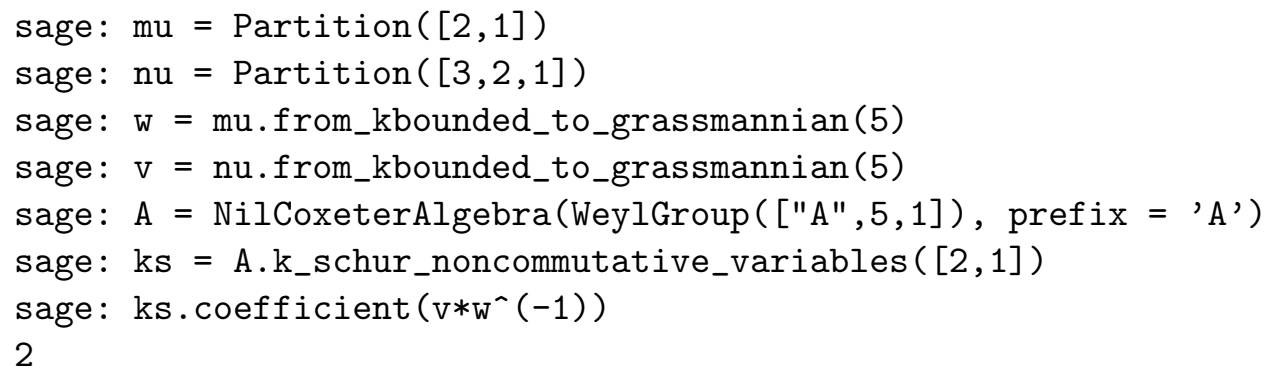

\subsection{Notes on references}

Note that in certain references (e.g. $[93,94,96])$ the notation for $\Lambda_{(k)}$ and $\Lambda^{(k)}$ are switched. We chose our convention to be consistent with [79], where the notation is motivated from the relation between these spaces and the homology/cohomology of the affine Grassmannian.

In reference [93, Conjecture 21] it is stated that the $k$-Schur functions defined using an algebraic definition satisfy the Pieri rule of Equation (2.19). Later references [95, 96] do use Equation (2.19) as the definition and prove properties from this point of view. The definition used in $[93,94]$ will be presented in the next section and is conjecturally equivalent to Equation (2.19) at $t=1$.

The proof of Theorem 2.22 is cited here as due to the affine insertion algorithm that was studied by Lam, Lapointe, Morse and Shimozono [81]. This algorithm will be discussed further in Section 6.3. A bijective algorithm is used to show that there is a duality between strong and weak orders on affine Grassmannians that is manifest in the Pieri rules for $k$-Schur functions and their duals.

When the details of affine Stanley symmetric functions are carried out in Chapter 3, there is a minor difference of notation; the affine nil-Coxeter algebra is denoted $\mathbb{A}_{n}$ in 
Section 2.5 and as $\mathbb{A}_{\mathrm{af}}$ in Chapter 3. In [133], Postnikov used the affine nil-Temperley-Lieb algebra, which is a quotient of the nil-Coxeter algebra, to provide a model for the quantum cohomology.

\section{Definitions of $k$-Schur functions}

In the previous section we defined $k$-Schur functions and their dual basis using the notions of strong and weak tableaux, but these are parameterless symmetric functions. In this section we will provide the original definition of $k$-Schur functions as well as several others that are conjecturally equivalent.

\subsection{Atoms as tableaux}

The origin of the tableaux definition (1.40) comes from identifying the $k$-Schur functions on the poset of standard tableaux ranked by the charge statistic. It is known that there is one Schur function in the expansion of the Macdonald symmetric functions for each standard tableau, so given that the $k$-Schur functions are these irreducible components of the Macdonald symmetric functions, it is natural to try to connect them with standard tableaux. These conjecturally symmetric functions were first given the name 'atoms' because they are the indecomposable pieces that come together to give Macdonald symmetric functions. They can be determined experimentally and from that point it is possible to conjecture beautiful properties that they possess.

Example 3.1. From Example 1.45 we 'know' that $A_{22}^{(2)}[X ; t]=s_{22}+t s_{31}+t^{2} s_{4}, A_{211}^{(2)}[X ; t]=$ $s_{211}+t s_{31}, A_{1111}^{(2)}[X ; t]=s_{1111}+t s_{211}+t^{2} s_{22}$. The following picture contains the standard tableaux of size 4 graded by charge. The tableau on the left has charge 0 , the one on the right has charge 6 and otherwise the left to right positions depends on the value of the charge. On this diagram we have circled the groups of these tableaux which represent the atoms:

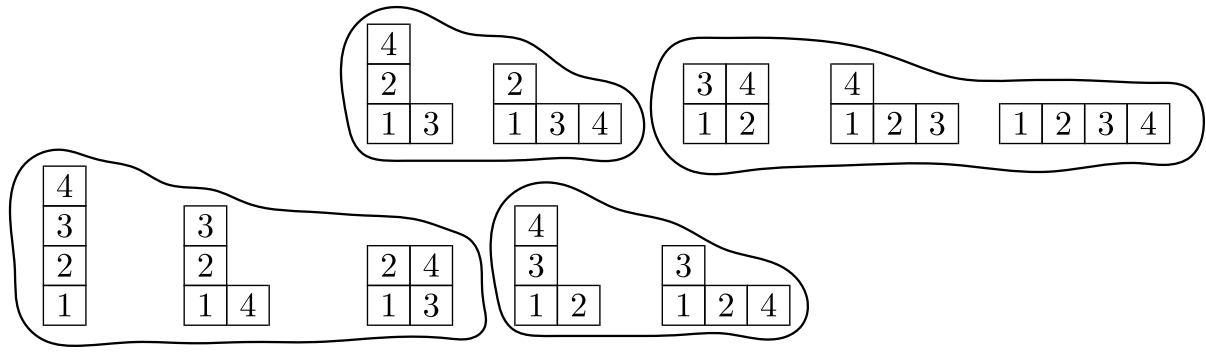

Moreover using the same method as in Example 1.45 to compute the 3-Schur functions, we obtain $s_{1111}^{(3)}[X ; t]=s_{1111}+t s_{211}, s_{211}^{(3)}=s_{211}+t s_{31}, s_{22}^{(3)}=s_{22}, s_{31}^{(3)}=s_{31}+t s_{4}$. We then 
picture these symmetric functions as groups of tableaux on the tableaux poset ranked by charge by placing a circle around the 'copies' of the 3-Schur functions:
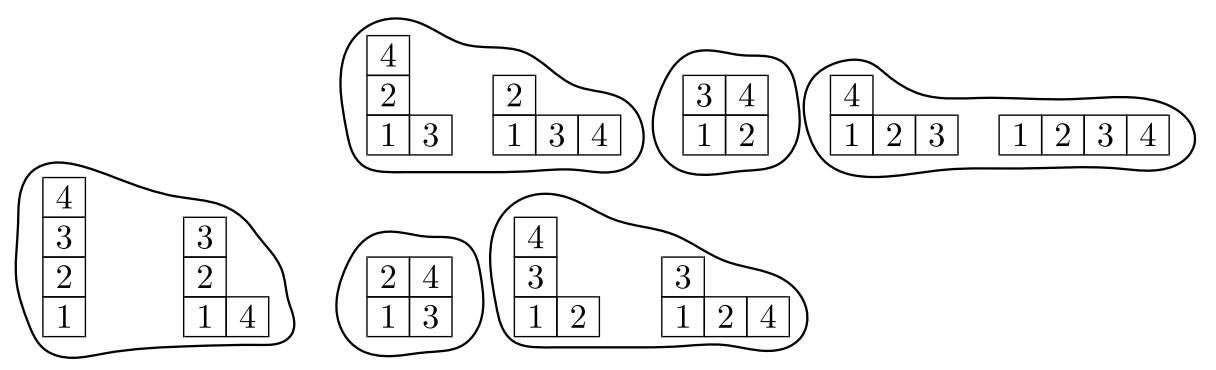

Notice in this example that the atoms of level $k=3$ are contained in those of level 2. We will see later that it is a property of $k$-Schur functions that the ones of level $k$ always expand positively in terms of $k$-Schur functions of level $k+1$.

At $k=4$ the $k$-Schur function $s_{\lambda}^{(4)}=s_{\lambda}$ for $\lambda \vdash 4$. Hence if we were to redraw the picture and circle the pieces representing the $k$-Schur functions, then each tableau would be in its own circle. This observation shows that moving from level 2 to 3 to 4 is that the circles representing the $k$-Schur functions break into smaller and smaller pieces.

The atoms can be computed by means of a recursive combinatorial procedure. We will describe each of the steps as operations on tableaux. The operators will act on a single tableau or a set of tableaux (depending on what is appropriate) and return a set of tableaux.

First we define $\sigma_{i}$ as the operator which takes a tableau with $a$ cells labeled with $i$ and $b$ cells labeled with $i+1$ and changes it into a tableau with $b$ cells labelled by $i$ and $a$ labelled with $i+1$. This is done by considering the reading word and placing a closed parenthesis ")" under each letter in the word labelled with an $i$ and and an open parenthesis "(" under each letter in the word labelled by an $i+1$. The word naturally has cells which have matching open and closed parentheses and the remainder of the parentheses are 'free.' Change the free open or closed parentheses and their corresponding labels so that in the end there are $b$ labels with $i$ and $a$ labels of $i+1$. The result of this operation is the tableau of the same shape, but with this new reading word. This operation is also known as the 'reflection along an $i$-string' in a crystal graph, see for example [105].

Example 3.2. Consider the action of $\sigma_{3}$ on the semi-standard tableau

\begin{tabular}{|l|l|l|l|l|l|l|l|l|}
\hline 4 & 5 & 5 & \multicolumn{9}{|c|}{} \\
\hline 3 & 3 & 4 & 6 & 6 & \multicolumn{3}{|c|}{} \\
\hline 2 & 2 & 3 & 3 & 3 & 5 & 6 & \multicolumn{2}{|c}{} \\
\hline 1 & 1 & 1 & 1 & 2 & 2 & 3 & 3 & 4 \\
\hline
\end{tabular}


Because there are four more $3 \mathrm{~s}$ in the word than there are $4 \mathrm{~s}$, we should change four of the label 3s to the label 4. Reading the entries in this tableau that are 3 or 4 only, the word is 4334333334 which, replacing 4 with (and 3 with ), corresponds to the word of parentheses $(())(())))$ )(. If we ignore all the parentheses which match up and change the rightmost four unmatched closed parentheses to open parentheses, this corresponds to the word ()$)()((((\rightarrow 4334344444$. Now this sequence of labels is placed back in the tableau in the positions of the labels of 3 and 4 , and the resulting tableau is

\begin{tabular}{|c|c|c|c|c|c|c|c|}
\hline 4 & 5 & 5 & & & & & \\
\hline 3 & 3 & 4 & 6 & 6 & & & \\
\hline 2 & 2 & 3 & 4 & 4 & & 6 & \\
\hline 1 & 1 & 1 & 1 & 2 & & 4 & \begin{tabular}{l|l|}
44 \\
\end{tabular} \\
\hline
\end{tabular}

The next operation is an analogue of the Pieri rule on tableaux. Define an operation $\mathbb{B}_{r}$ for $r \geq 1$ which adds a horizontal strip of size $r$ to the tableau in all possible ways and then acts by operators $\sigma_{i}$ to change the weight so that there are $r$ labels of 1 . That is, if we take a tableau $T$ of shape $\lambda \vdash m$,

$$
\mathbb{B}_{r}(T)=\left\{\sigma_{1} \sigma_{2} \cdots \sigma_{\ell(\mu)}(T \subseteq \mu): \mu / \lambda \text { is a horizontal strip of size } r\right\}
$$

where $T \subseteq \mu$ represents the semi-standard tableau $T$ as a sequence of partitions with an additional partition $\mu$ attached. 
Example 3.3. Consider the action of $\mathbb{B}_{3}$ on the tableau $\begin{aligned} & \frac{3}{3} \\ & \frac{2}{1}\end{aligned}$

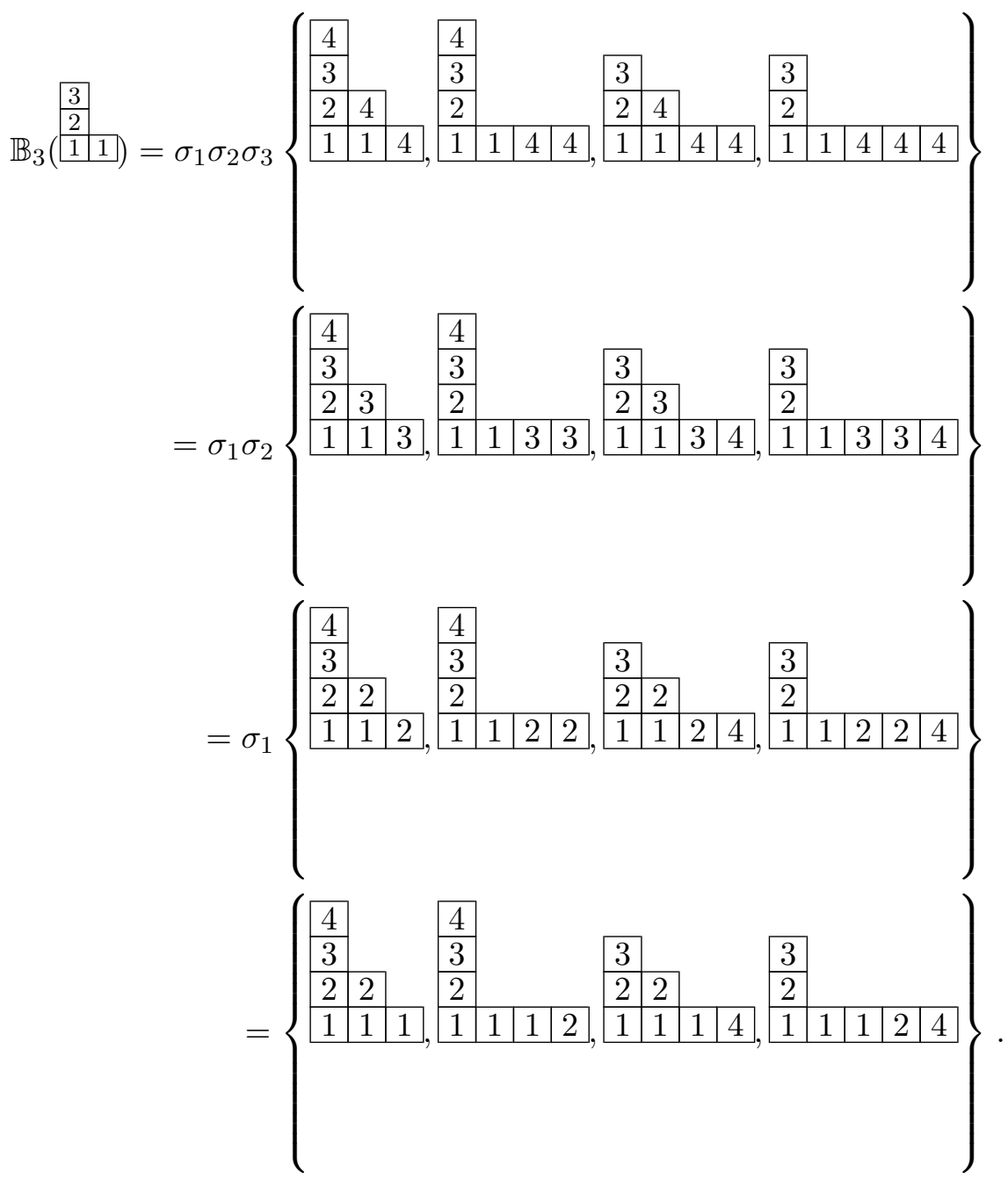

The last notion that we need is that of a katabolizable tableau. To introduce this defintion we need the notion of jeu de taquin, Knuth equivalence or the Robinson-SchenstedKnuth algorithm. We introduce a generalization of the Robinson-Schensted-Knuth algorithm in Section 6. We assume here that the reader is familiar with this notion (and if not then one can skip ahead a few sections or consult [140] for example).

Let $T$ be a tableau with reading word $w$. The definition that $T$ is katabolizable with respect to a sequence of partitions $\lambda^{(*)}=\left(\lambda^{(1)}, \lambda^{(2)}, \ldots, \lambda^{(r)}\right)$ is recursive. It is required that $r=\ell\left(\lambda^{(1)}\right)$. Then let $w=u v$, where $u$ is the largest subword of $w$ that does not contain an $r$. Let $v^{\prime}$ be $v$ with all letters 1 through $r$ deleted. We say that $T$ is katabolizable 
with respect to $\lambda^{(*)}$ if $T$ contains as a subtableau the semi-standard tableau of shape $\lambda^{(1)}$ and weight $\lambda^{(1)}$ and if $\operatorname{RSK}\left(v^{\prime} u\right)=(P, Q)$ where $P$ is a tableau which is $\left(\lambda^{(2)}, \ldots, \lambda^{(r)}\right)$ katabolizable with the labels shifted by $r$.

Example 3.4. Consider the tableau

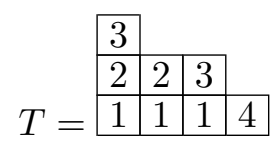

which is katabolizable with respect to the sequence of partitions $((3,2),(2,1))$. This is because $r=\ell\left(\lambda^{(1)}\right)=2$. Furthermore, the reading word of $T$ is $w=32231114=u v$, where $u=3$ and $v=2231114$. Then $v^{\prime}=34$ and the $P$ tableau of $\operatorname{RSK}\left(v^{\prime} u\right)$ is $\frac{4}{3} 3$.

Now $T$ is not katabolizable with respect to the sequence $((3),(2,2),(1))$. The reason is that in this case $u=3223$ and $v=1114$, so that $v^{\prime}=4$. Then the $P$ tableau of $\operatorname{RSK}\left(v^{\prime} u\right)$ is

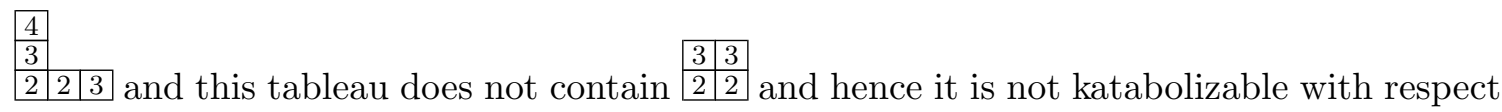
to the sequence $((2,2),(1))$.

Recall that we introduced the notion of the $k$-split of a partition in (1.2). Now define an operator on tableaux $\mathbb{K}^{\rightarrow k}$ that acts on a set of tableaux with partition weight $\lambda$ such that $\mathbb{K}^{\rightarrow k}$ kills all tableaux that are not katabolizable with respect to the $k$-split of $\lambda$ and keeps the ones that are katabolizable.

Example 3.5. Consider the action of $\mathbb{K}^{\rightarrow 3}$ on the following set of semi-standard tableaux with weight $(2,1,1,1,1)$. Since $(2,1,1,1,1) \rightarrow 3=((2,1),(1,1,1))$, a tableau will only survive if it contains $\begin{array}{ll}2 & 1 \\ \frac{1}{1} & 1\end{array}$ as a subtableau.

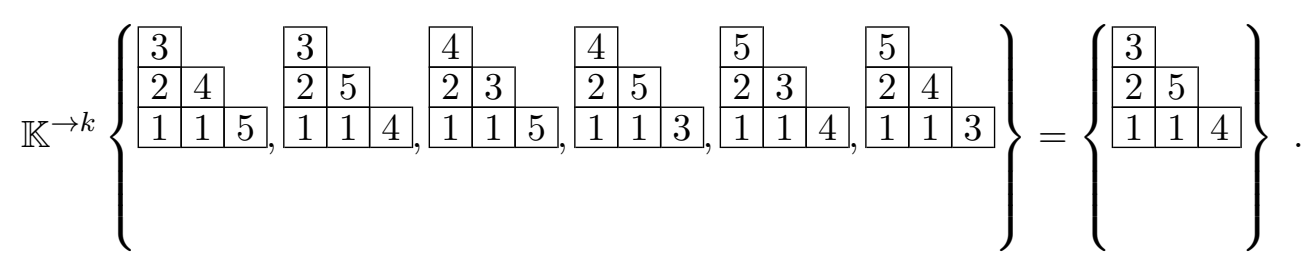

Given a $k$-bounded partition $\lambda$, we define the $k$-atom $\mathbb{A}_{\lambda}^{(k)}$ as a set of tableaux which are computed recursively as

$$
\mathbb{A}_{\lambda}^{(k)}=\mathbb{K}^{\rightarrow k} \mathbb{B}_{\lambda_{1}} \mathbb{A}_{\left(\lambda_{2}, \lambda_{3}, \ldots, \lambda_{\ell(\lambda)}\right)}^{(k)} .
$$




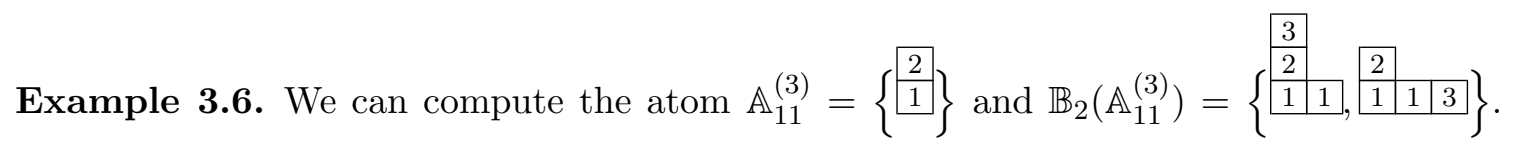
The atom $\mathbb{A}_{211}^{(3)}=\left\{\begin{array}{l|l|l|l|l|}\hline 3 & 2 \\ \hline & 1 & 1\end{array}, \begin{array}{lll}1 & 1 & 3 \\ \hline\end{array}\right\}$ because each of these tableaux survives the operator $\mathbb{K}^{\rightarrow 3}$. As we have seen in Example 3.3, we know what the action of $\mathbb{B}_{3}$ on the first tableau

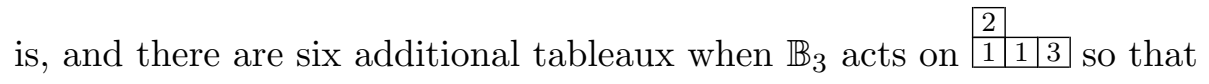

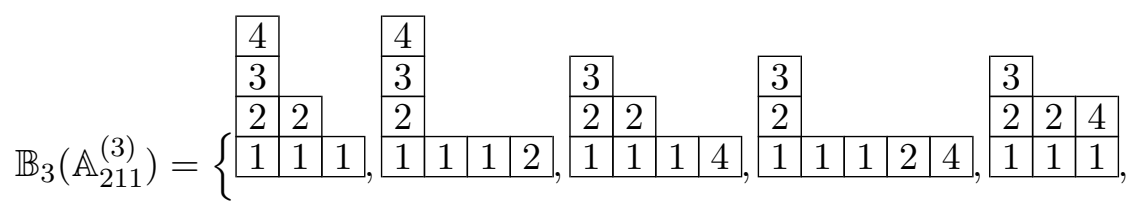

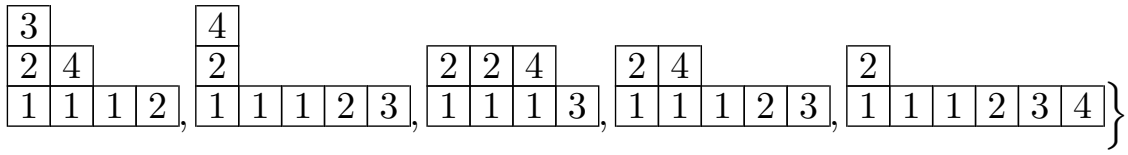

It is an unusual situation, but all 10 of these tableaux are katabolizable with respect to the sequence $(3,2,1,1)^{\rightarrow 3}=((3),(2,1),(1))$ and hence survive the operator $\mathbb{K}^{\rightarrow 3}$.

If on the other hand, we do the computation with $k=4$, we find

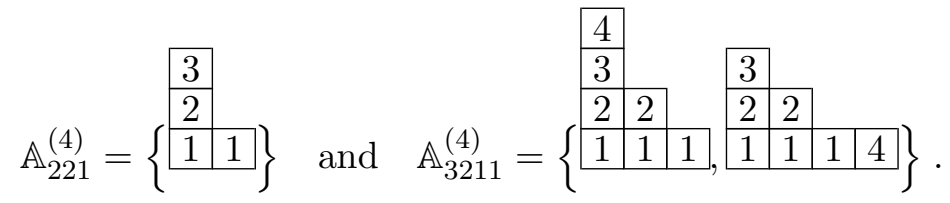

We can check (3.3) in SAGE via

$$
\begin{aligned}
& \text { sage: } 1 a=\operatorname{Partition}([3,2,1,1]) \\
& \text { sage: } 1 \text { a.k_atom }(4) \\
& {[[[1,1,1],[2,2],[3],[4]],[[1,1,1,4],[2,2],[3]]]}
\end{aligned}
$$

Now we define the symmetric function $A_{\lambda}^{(k)}[X ; t]$ in terms of the set of tableaux $\mathbb{A}_{\lambda}^{(k)}$ as

$$
A_{\lambda}^{(k)}[X ; t]=\sum_{T \in \mathbb{A}_{\lambda}^{(k)}} t^{\operatorname{charge}(T)} s_{\text {shape }(T)} .
$$

Example 3.7. The charge of the tableau of shape $(3,2,1,1)$ in $(3.3)$ is 0 , whereas the charge of the tableau of shape $(4,2,1)$ is 1 . Hence we obtain

$$
A_{3211}^{(4)}[X ; t]=s_{3211}+t s_{421}
$$


The element $A_{3211}^{(3)}[X ; t]$ is a generating function for the tableaux in (3.2) with a term of the form $t$ raised to the charge times the Schur function indexed by the shape. By computing the charge of each of these tableaux we determine

$$
A_{3211}^{(3)}[X ; t]=s_{3211}+t s_{4111}+\left(t+t^{2}\right) s_{421}+t s_{331}+\left(t^{2}+t^{3}\right) s_{511}+t^{2} s_{43}+t^{3} s_{52}+t^{4} s_{61} .
$$

The atoms by this definition are conjectured to be a basis of the space

$$
\begin{aligned}
\Lambda_{(k)}^{t} & =\mathcal{L}_{\mathbb{Q}(q, t)}\left\{H_{\lambda}[X ; q, t]: \lambda_{1} \leq k\right\} \\
& =\mathcal{L}_{\mathbb{Q}(q, t)}\left\{s_{\lambda}\left[\frac{X}{1-t}\right]: \lambda_{1} \leq k\right\} \\
& =\mathcal{L}_{\mathbb{Q}(q, t)}\left\{Q_{\lambda}^{\prime}[X ; t]: \lambda_{1} \leq k\right\}
\end{aligned}
$$

However, no proof that these functions are even elements of this space currently exists.

\subsection{A symmetric function operator definition}

In this section we present a conjecturally equivalent definition for $k$-Schur functions that is in similar spirit to the atom description given in the previous section, but now with an algebraic flavor.

In Section 1.7 we defined the operator $\mathbf{B}_{m}$ which has the property that $\mathbf{B}_{m}\left(Q_{\lambda}^{\prime}[X ; t]\right)=$ $Q_{(m, \lambda)}^{\prime}[X ; t]$. We now define a new operator in terms of the $\mathbf{B}_{m}$ as

$$
\mathbf{B}_{\lambda}:=\prod_{1 \leq i<j \leq \ell(\lambda)}\left(1-t R_{i j}\right) \mathbf{B}_{\lambda_{1}} \mathbf{B}_{\lambda_{2}} \cdots \mathbf{B}_{\lambda_{\ell(\lambda)}}
$$

where

$$
R_{i j}\left(\mathbf{B}_{\mu_{1}} \mathbf{B}_{\mu_{2}} \cdots \mathbf{B}_{\mu_{\ell(\mu)}}\right)=\mathbf{B}_{\mu_{1}} \mathbf{B}_{\mu_{2}} \cdots \mathbf{B}_{\mu_{i}+1} \cdots \mathbf{B}_{\mu_{j}-1} \cdots \mathbf{B}_{\mu_{\ell(\mu)}} .
$$

Alternatively $\mathbf{B}_{\lambda}$ is also given by the equation

$$
\mathbf{B}_{\lambda}=\sum_{\nu, \mu} c_{\mu \lambda}^{\nu} s_{\nu}[X] s_{\mu}[X(t-1)]^{\perp}
$$

where the sum is over partitions $\nu$ and $\mu$ such that $\ell(\nu) \leq \ell(\lambda)$ and $\ell(\mu) \leq \ell(\lambda)$ and $c_{\mu \lambda}^{\nu}=\left\langle s_{\mu} s_{\lambda}, s_{\nu}\right\rangle$ is the Littlewood-Richardson coefficient from (1.25).

The 'parabolic' Hall-Littlewood symmetric functions that we will need are defined in terms of these operators. For a sequence of partitions $\lambda^{(*)}=\left(\lambda^{(1)}, \lambda^{(2)}, \ldots, \lambda^{(d)}\right)$ define the symmetric function

$$
H_{\lambda^{(*)}}[X ; t]=\mathbf{B}_{\lambda^{(1)}} \mathbf{B}_{\lambda^{(2)}} \cdots \mathbf{B}_{\lambda^{(d)}}(1) .
$$

It is easy to see from (3.7) that when $t=1$, we have $H_{\lambda^{(*)}}[X ; 1]=s_{\lambda^{(1)}} s_{\lambda^{(2)}} \cdots s_{\lambda^{(d)}}$. The index set of these symmetric functions is much larger than the set of partitions, 
so that these elements are not linearly independent. In fact, these symmetric function interpolate between the Hall-Littlewood symmetric functions and the Schur functions since $H_{(\lambda)}[X ; t]=s_{\lambda}$, that is, the element indexed by a list containing exactly one partition, and $H_{\left(\left(\lambda_{1}\right),\left(\lambda_{2}\right), \ldots,\left(\lambda_{\ell(\lambda)}\right)\right)}[X ; t]=Q_{\lambda}^{\prime}[X ; t]$, that is, the element indexed by a list where each partition is a single part of $\lambda$.

There is a conjectured combinatorial interpretation for the expansion of $H_{\lambda^{(*)}}[X ; t]$ (see [148]) if $\lambda^{(*)}$ is a sequence of partitions such that the concatenation of the partitions in $\lambda^{(*)}$ is a partition (i.e. if for each of the adjacent partitions in $\lambda^{(*)}$ we have $\lambda_{\ell\left(\lambda^{(i)}\right)}^{(i)} \geq \lambda_{1}^{(i+1)}$ ). In this case

$$
H_{\lambda^{(*)}}[X ; t]=\sum_{T} t^{\operatorname{charge}(T)} s_{\text {shape }(T)}
$$

where the sum is over all tableaux $T$ which are katabolizable with respect to $\lambda^{(*)}$.

The first algebraic definition of $k$-Schur functions requires an intermediary basis; the $k$-split basis for $\Lambda_{(k)}^{t}$. This basis is made up of, for each $k$-bounded partition $\lambda, G_{\lambda}^{(k)}[X ; t]=$ $H_{\lambda \rightarrow k}[X ; t]$.

Sage Example 3.8. The 3 -split basis element $G_{3211}^{(3)}$ is a $t$-analogue of the product of $s_{3} s_{21} s_{1}$. The operators $\mathbf{B}_{\lambda}$ are programmed in SAGE and we may calculate $\mathbf{B}_{(3)} \mathbf{B}_{(2,1)} \mathbf{B}_{(1)}(1)$ in the following steps:

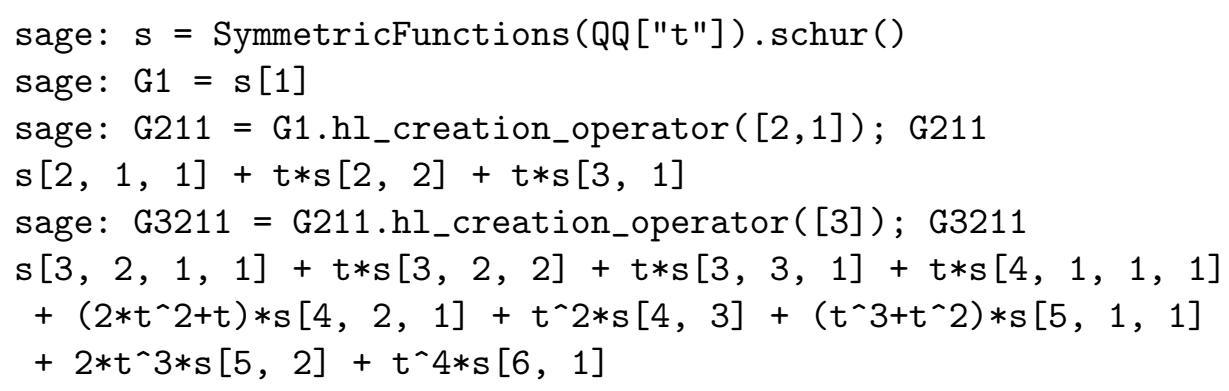

This calculation shows that $\mathbf{B}_{(2,1)}\left(s_{1}\right)=s_{\theta}+t s^{+t s_{\square}}$ and

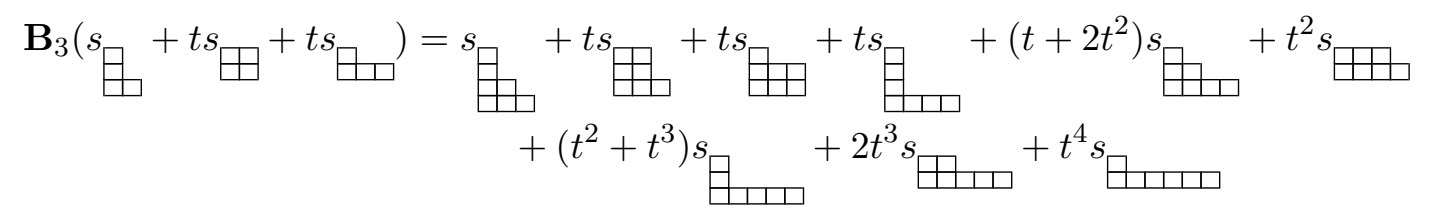

and this expression is equal to $G_{3211}^{(3)}$.

Then, to arrive at the $k$-Schur functions, a second operator is needed. Let $T_{i}^{(k)}$ be an operator on symmetric functions defined so that

$$
T_{i}^{(k)}\left(G_{\lambda}^{(k)}[X ; t]\right)= \begin{cases}G_{\lambda}^{(k)}[X ; t] & \text { if } \lambda_{1}=i \\ 0 & \text { otherwise }\end{cases}
$$


For $k$-bounded partition $\lambda$, the elements $\tilde{A}_{\lambda}[X ; t]$ were defined in [93] by a recursive algorithm; if $\ell(\lambda)=1$ and $r \leq k$, then $\tilde{A}_{(r)}[X ; t]=s_{(r)}$. Otherwise we set for $\lambda_{1} \leq m \leq k$,

$$
\tilde{A}_{\left(m, \lambda_{1}, \lambda_{2}, \ldots, \lambda_{\ell(\lambda)}\right.}^{(k)}[X ; t]=T_{m}^{(k)} \mathbf{B}_{m} \tilde{A}_{\lambda^{(k)}}[X ; t] .
$$

Example 3.9. The 3 -split of $(2,1,1)$ is $(2,1,1) \rightarrow 3=((2,1),(1))$, hence

$$
\tilde{A}_{211}^{(3)}=T_{2}^{(3)} \mathbf{B}_{2}\left(s_{11}\right)=T_{2}^{(3)}\left(s_{211}+t s_{31}\right)=T_{2}^{(3)}\left(G_{211}^{(3)}-G_{22}^{(3)}\right)=s_{211}+t s_{31} .
$$

Moreover, we may calculate using Example 3.8 that

$$
\begin{aligned}
\mathbf{B}_{3}\left(\tilde{A}_{211}^{(3)}\right) & =s_{3211}+t s_{331}+t s_{4111}+\left(t+t^{2}\right) s_{421}+t^{2} s_{43}+\left(t^{2}+t^{3}\right) s_{51}+t^{3} s_{52}+t^{4} s_{61} \\
& =G_{3211}^{(3)}-t s_{322}-t^{2} s_{421}-t^{3} s_{52} .
\end{aligned}
$$

We can also determine that $G_{322}^{(3)}=s_{322}+t s_{421}+t^{2} s_{52}$ (using the same techniques as in Example 3.8), so that

$$
\tilde{A}_{3211}^{(3)}=T_{3}^{(3)} \mathbf{B}_{3}\left(\tilde{A}_{211}^{(3)}\right)=G_{3211}^{(3)}-t G_{322}^{(3)} .
$$

This (not coincidently) is equal to $A_{3211}^{(3)}[X ; t]$ as was calculated in Example 3.7.

The algebraic operations mimic those of the combinatorial definition defined in the previous section and so it is important to emphasize that the combinatorial and algebraic definitions are equivalent.

Conjecture 3.10. For $k>0$ and a $k$-bounded partition $\lambda$,

$$
A_{\lambda}^{(k)}[X ; t]=\tilde{A}_{\lambda}^{(k)}[X ; t] .
$$

\subsection{Weak tableaux II}

We have seen that a fruitful characterization for $k$-Schur functions (without parameter $t$ ) is given by inverting (2.14); for $\mu_{1} \leq k$,

$$
h_{\mu}=\sum_{\lambda: \lambda_{1} \leq k} K_{\lambda \mu}^{(k)} s_{\lambda}^{(k)},
$$

where the weak Kostka numbers $K_{\lambda \mu}^{(k)}$ count weak $k$-tableaux. Here we present $k$-Schur functions that reduce to these parameterless $k$-Schur functions when $t=1$. The method is to introduce weak Kostka-Foulkes polynomials as polynomials in $\mathbb{N}[t]$ defined by refining the charge statistic to a statistic that associates a non-negative integer called the $k$-charge to each $k$-tableau. Then setting

$$
K_{\lambda \mu}^{(k)}(t)=\sum_{\substack{\operatorname{shape}(T)=\mathfrak{c}(\lambda) \\ \operatorname{weight}(T)=\mu}} t^{\operatorname{kcharge}(T)},
$$


it happens that $K_{\lambda \lambda}^{(k)}(t)=1$ and since there are no $k$-tableaux of shape $\mathfrak{c}(\lambda)$ and weight $\mu$ when $\mu>\lambda$, the $k$-charge matrix $K_{\lambda \mu}^{(k)}(t)$ is unitriangular. So, in the spirit of (3.12),

$$
Q_{\mu}^{\prime}[X ; t]=\sum_{\lambda} K_{\lambda \mu}^{(k)}(t) \tilde{s}_{\lambda}^{(k)}[X ; t]
$$

characterizes the functions $\left\{\tilde{s}_{\lambda}^{(k)}[X ; t]\right\}$.

There are several different characterizations for $k$-charge. We give here two distinct formulations defined directly on $k$-tableaux, discovered by Lapointe-Pinto and Morse [99, 36]. There are other formulations including one on $\alpha$-factorizations, one on an object called affine Bruhat countertableau [35, 36], and in relation with the energy function on Kirillov-Reshetikhin crystals [126].

The $k$-charge statistic on $k$-tableaux is first described in the standard case since it is in these terms that we define it for semi-standard $k$-tableaux. Important to the definition is a number $\operatorname{diag}\left(c_{1}, c_{2}\right)$, associated to cells $c_{1}$ and $c_{2}$ in a $(k+1)$-core, defined to be the number of diagonals of residue $x$ that are strictly between $c_{1}$ anc $c_{2}$ where $x$ is the residue of the lower cell. When it is well-defined to do so, functions defined with a cell as input can instead take a letter as input. For example, in a standard $k$-tableau it is natural to discuss the residue of a specific letter (since any cell containing that letter has the same residue) instead of the residue of a specific cell.

Definition 3.11. Given a standard $k$-tableau $T$ on $m$ letters, put a bar on the topmost occurrence of letter $r$, for each $r=1, \ldots, m$. Define the index of $T$, starting from $I_{1}=0$, by

$$
I_{r}= \begin{cases}I_{r-1}+1+\operatorname{diag}(\bar{r}, \overline{r-1}) & \text { if } \bar{r} \text { is east of } \overline{r-1} \\ I_{r-1}-\operatorname{diag}(\bar{r}, \overline{r-1}) & \text { otherwise }\end{cases}
$$

for $r=2, \ldots, m$. The $k$-charge of $T$ is the sum of entries in $I(T)$, denoted by $\operatorname{kcharge}(T)$.

Example 3.12. For $k=3$,

$$
T=\begin{array}{|l|l|l|l|l|}
\hline 4_{2} & & \\
\hline 2_{3} & 6_{0} & & \\
\hline 1_{0} & 3_{1} & 4_{2} & 5_{3} & 6_{0} \\
\hline
\end{array} \Longrightarrow I(T)=[0,0,1,1,3,3] \quad \Longrightarrow \operatorname{kcharge}(T)=8 .
$$

It is not immediately clear that the $k$-charge is a non-negative integer and it is sometimes helpful to use a different formulation of $k$-charge. Let $T_{\leq x}$ denote the subtableau obtained by deleting all letters larger than $x$ from $T$.

Definition 3.13. Given a $k$-tableau $T$, the $T$-residue order of $\{0, \ldots, k\}$ is defined by

$$
x>x-1>\cdots>0>k>\cdots>x+1,
$$

where $x$ is the residue of the highest addable corner of $T$. Note that $x=1-\ell(\lambda)(\bmod k+1)$, for $\lambda$ the shape of $T$. 
Example 3.14. With $k=3$, consider

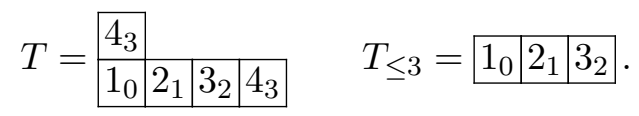

The $T_{\leq 3}$-residue order is $3>2>1>0$ and the $T$-residue order is $2>1>0>3$.

Given a standard $k$-tableau $T$ on $m$ letters, define the index $J(T)=\left[J_{1}, \ldots, J_{m}\right]$, starting from $J_{1}=0$, by setting for $r=2, \ldots, m$,

$$
J_{r}= \begin{cases}J_{r-1}+1 & \text { if } \operatorname{res}(r)>\operatorname{res}(r-1) \\ J_{r-1} & \text { otherwise }\end{cases}
$$

under $T_{\leq r}$-residue order (see Example 3.16).

Proposition 3.15. For a standard $k$-tableau $T$ of shape $\lambda$,

$$
\operatorname{kcharge}(T)=\sum_{r}\left(J_{r}(T)+\operatorname{diag}\left(c_{r}, c^{(r)}\right)\right),
$$

where $c_{r}$ is the highest cell containing an $r$ and $c^{(r)}=\left(\ell\left(\operatorname{shape}\left(T_{\leq r}\right)\right)+1,1\right)$.

Example 3.16. For $k=3$,

$$
T=\begin{array}{|l|l|l|l|l|}
\hline 4_{2} & & &
\end{array} \quad \Longrightarrow J(T)=[0,0,1,1,2,3], \operatorname{diag}\left(c_{5},(4,1)\right)=1 \Longrightarrow \operatorname{kcharge}(T)=8 .
$$

Remark 3.17. Given a standard $k$-tableau of shape $\lambda$ where $k \geq h(\lambda)$, the index conditions (3.14) and (3.15) both reduce to (1.37). Thus, since a diagonal of residue $x$ occurs at most once in $\lambda$ for any $x, k$-charge reduces to charge.

As with the charge of Lascoux and Schützenberger, we extend the definition of $k$ charge to semi-standard $k$-tableaux by successively computing on an appropriate choice of standard sequences. The trick is to introduce a method for making this choice in $k$ tableaux.

Definition 3.18. From an $x$ (of some residue $i$ ) in a semi-standard $k$-tableau $T$, the appropriate choice of $x+1$ will be determined by choosing its residue from the set $A$ of all $(k+1)$-residues labelling $x+1$ 's. Reading counter-clockwise from $i$, this choice is the closest $j \in A$ on a circle labelled clockwise with $0,1, \ldots, k$.

Remark 3.19. Definition 3.18 reduces to Definition 1.41 when $T$ is a semi-standard tableau of shape $\lambda$ and $k \geq h(\lambda)$. In particular, consider $x$ of $(k+1)$-residue $i$ in $T$. Note that $h(\lambda) \leq k$ implies there is a unique cell $c$ of residue $i$ that contains $x$. Let $j$ be the first 
entry on the circle reading counter-clockwise from $i$ that is a residue of a cell containing $x+1$. If there is an $x+1$ above $c$, then the south-easternmost cell containing an $x+1$ that is above $c$ has residue $j$ since there are no $x+1$ 's of a residue counter-clockwise between $i$ and $j$. If there are none above $c$, then for the same reason, the south-easternmost cell containing an $x+1$ has residue $j$.

In the semistandard case, we need to be more specific about the residue order used in (3.15). The letters $r$ that occur in the tableau are ordered with respect to the standard subsequence they belong to under Definition 3.18. So $r$ in the first chosen standard subword is bigger than the one from the second standard subword etc. Each letter $r$ has its own distinct residue $i$. The $J$-index of (3.15) should be computed with respect to the $T_{\leq r_{i}}{ }^{-}$ residue order when dealing with letter $r_{i}$.

Example 3.20. With $k=4$ and weight $(2,2,2,2,2,2,1)$ :
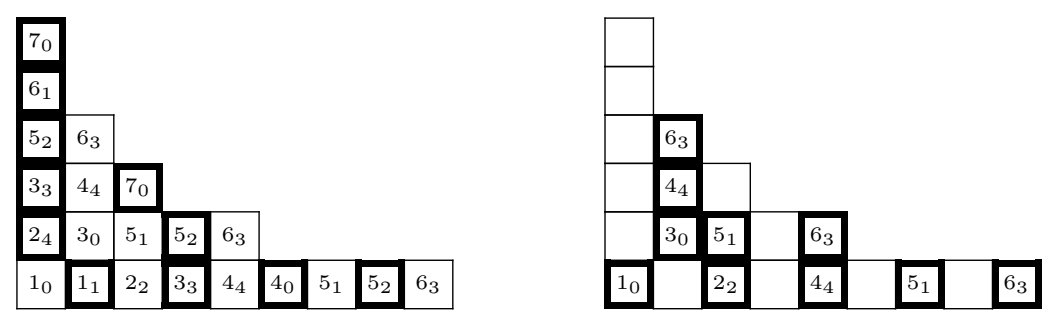

Note that for example

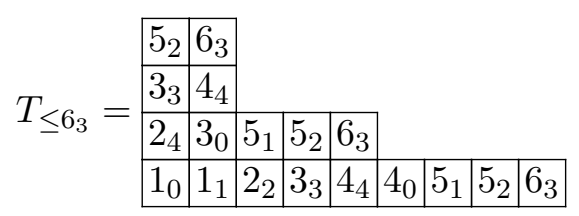

so that the highest addable cell in $T_{\leq 6_{3}}$ has residue 1 . We have

$$
\begin{aligned}
& J=[0,0,0,1,1,1,1] \text { and } \operatorname{diag}\left(c_{4}, c^{(4)}\right)=1 \quad J=[0,1,1,1,2,2] \\
& I=[0,0,0,2,1,1,1] \operatorname{since} \operatorname{diag}(\overline{3}, \overline{4})=1, \operatorname{diag}(\overline{4}, \overline{5})=1 \quad I=[0,1,1,1,2,2]
\end{aligned}
$$

Using either index $I$ or $J$, we find that the $k$-charge of $T$ is 12 .

Sage Example 3.21. We verify the above example in SAGE:

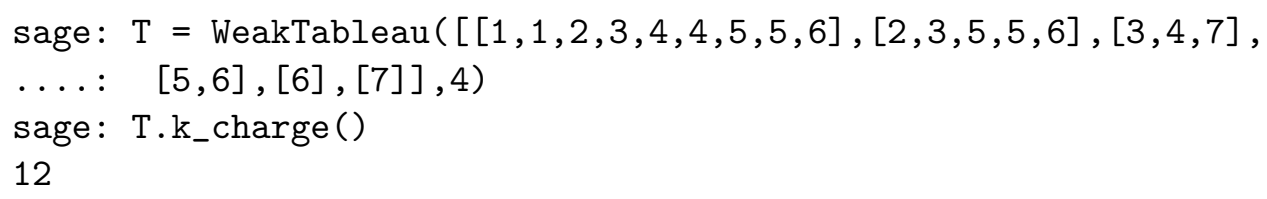

We can also demonstrate an example of Equation (3.13) using SAGE. 


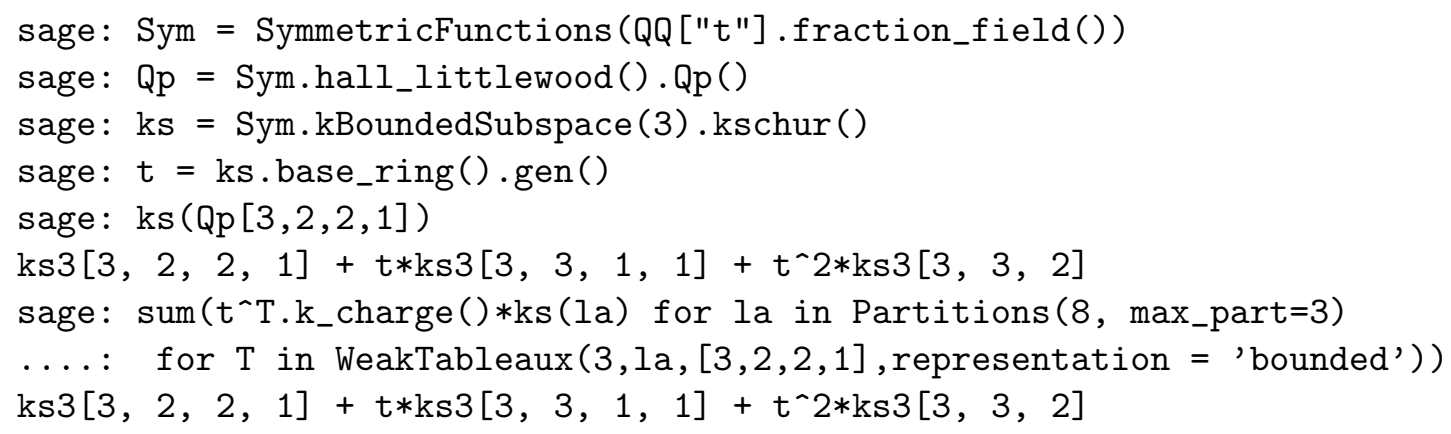

\subsection{Strong tableaux II}

The definition of the $k$-Schur functions in terms of strong marked tableaux as in (2.29) also has a version with a parameter $t$. For this definition we need to define the spin of a strong marked ribbon. Recall that if $\tau$ and $\kappa$ are $(k+1)$-cores such that $\tau \Rightarrow_{k} \kappa$, then $\kappa / \tau$ is a skew partition which consists of several copies of connected components which are ribbons of the same size and shape. Let $h$ represent the height of one of these ribbons (that is that it occupies $h$ rows). Now a strong marked cover consists of the skew partition $\kappa / \tau$ and a marking $c$ of one of the connected components. If there are $r$ connected components in $\kappa / \tau$, then the spin of a marked cover is equal to $(h-1) \times r$ plus the number of ribbons which are above the marked one.

The spin of a strong marked tableau, $\kappa^{(0)}=\emptyset \Rightarrow_{k} \kappa^{(1)} \Rightarrow_{k} \kappa^{(2)} \Rightarrow_{k} \cdots \Rightarrow_{k} \kappa^{(m)}$ with markings $c_{1}, c_{2}, \ldots, c_{m}$ is the sum of the spins of the strong marked ribbons $\kappa^{(i)} / \kappa^{(i-1)}$ with marking $c_{i}$.

Example 3.22. Recall from Example 2.23 the marked semi-standard tableau with $k=3$ :

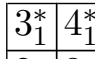

$$
\begin{aligned}
& \begin{array}{lll}
2_{2} & 3_{3} \\
\hline
\end{array} \\
& \begin{array}{|l|l|l|l|}
\hline 2_{1}^{*} & 3_{2} & 3_{2}^{*} & 4_{1} \\
\hline 1^{*} & 1^{*} & 2^{*} & 3^{*} \\
\hline
\end{array} \\
& \begin{array}{|l|l|l|l|}
\hline 1_{1}^{*} & 1_{2}^{*} & 2 & 3 \\
\hline
\end{array}
\end{aligned}
$$

There is a contribution of 1 to the spin for the ribbon of cells labelled by $2_{2}$ and there is a contribution of 1 due to the labeling of the lower occurrence of $3_{3}$. Therefore the total spin of this tableau is 2 .

The $k$-Schur function (this time with a $t$ ) in terms of strong tableaux are defined as (see also [81, Conjecture 9.11])

$$
s_{\lambda}^{(k)}[X ; t]=\sum_{\mu \vdash|\lambda|} \sum_{\left(\kappa^{(*)}, c_{*}\right)} t^{\operatorname{spin}\left(\kappa^{(*)}, c_{*}\right)} m_{\mu},
$$

where the sum is over all strong marked tableaux $\left(\kappa^{(*)}, c_{*}\right)$ of shape $\lambda$ and weight $\mu$. 
Example 3.23. For this definition, there is a reason to choose a smaller example for computation. We have thus far used as our running example the 3-Schur function indexed by the partition $(3,2,1,1)$. For the coefficient of the monomial $m_{1111111}$ there are 210 strong marked tableaux. To choose a smaller example we take as an example the 3-bounded partition $(3,1,1)$ which corresponds to the 4 -core $(4,1,1)$ which has 10 strong marked tableaux in total. The strong partial order on 4-cores contains the following interval (see Figure 2.5):

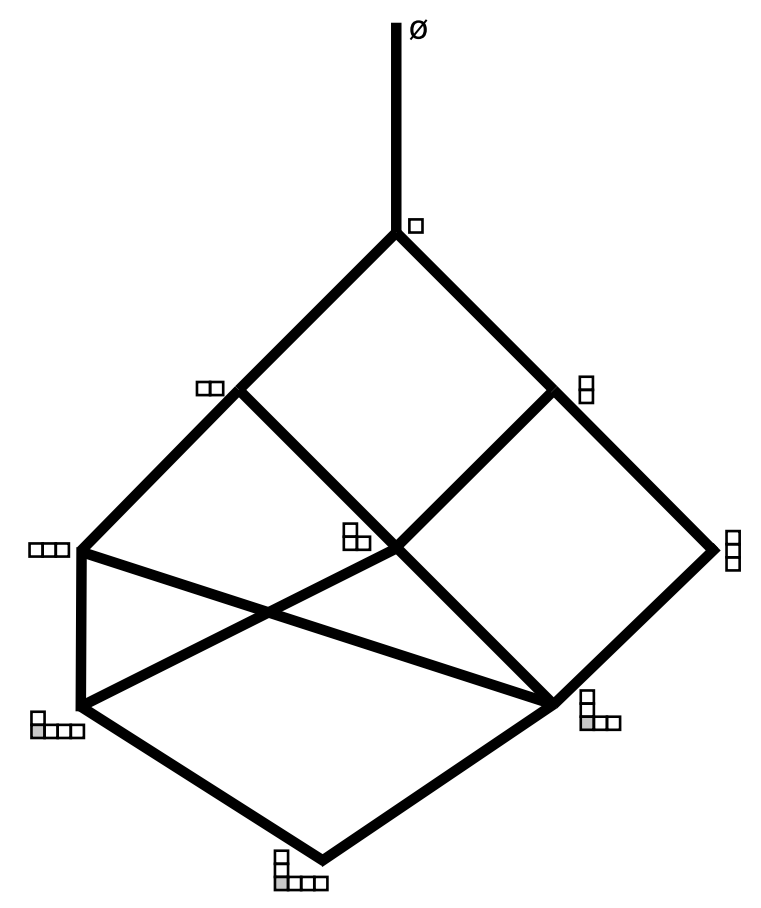

These correspond to the following 10 marked strong standard tableaux:

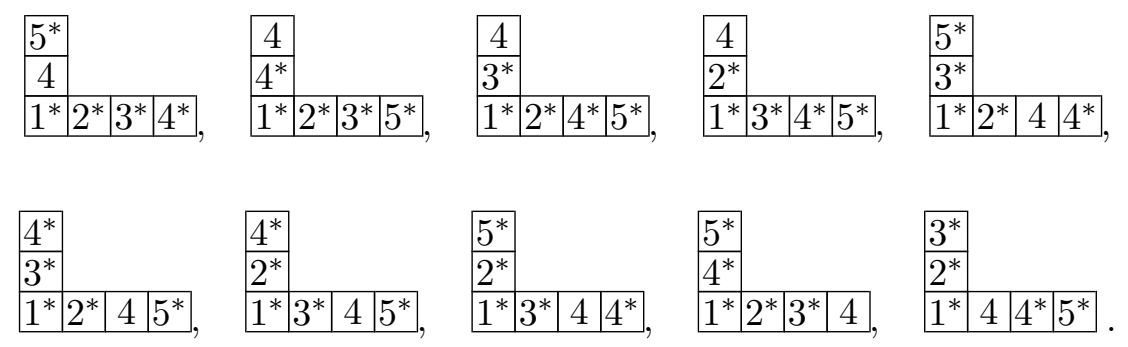

The first four of these strong marked standard tableaux have spin equal to 1 and the remaining 6 have spin equal to 0 . There is a semi-standard tableau of weight $\mu$ which corresponds to the standard tableau if the cells labeled $1,2, \ldots, \mu_{1}$ form a strong marked 
horizontal strip, $\mu_{1}+1, \mu_{1}+2, \ldots, \mu_{1}+\mu_{2}$ form another strong marked horizontal strip, etc.. From these 10 strong marked standard tableaux it is possible to read off that

$s_{311}^{(3)}[X ; t]=t m_{41}+t m_{32}+(1+2 t) m_{311}+(1+2 t) m_{221}+(3+3 t) m_{2111}+(6+4 t) m_{111111}$.

Sage Example 3.24. In this example we will show how SAGE can be used to compute the monomial expansion of the $k$-Schur function by computing a statistic for each strong $k$-tableau.

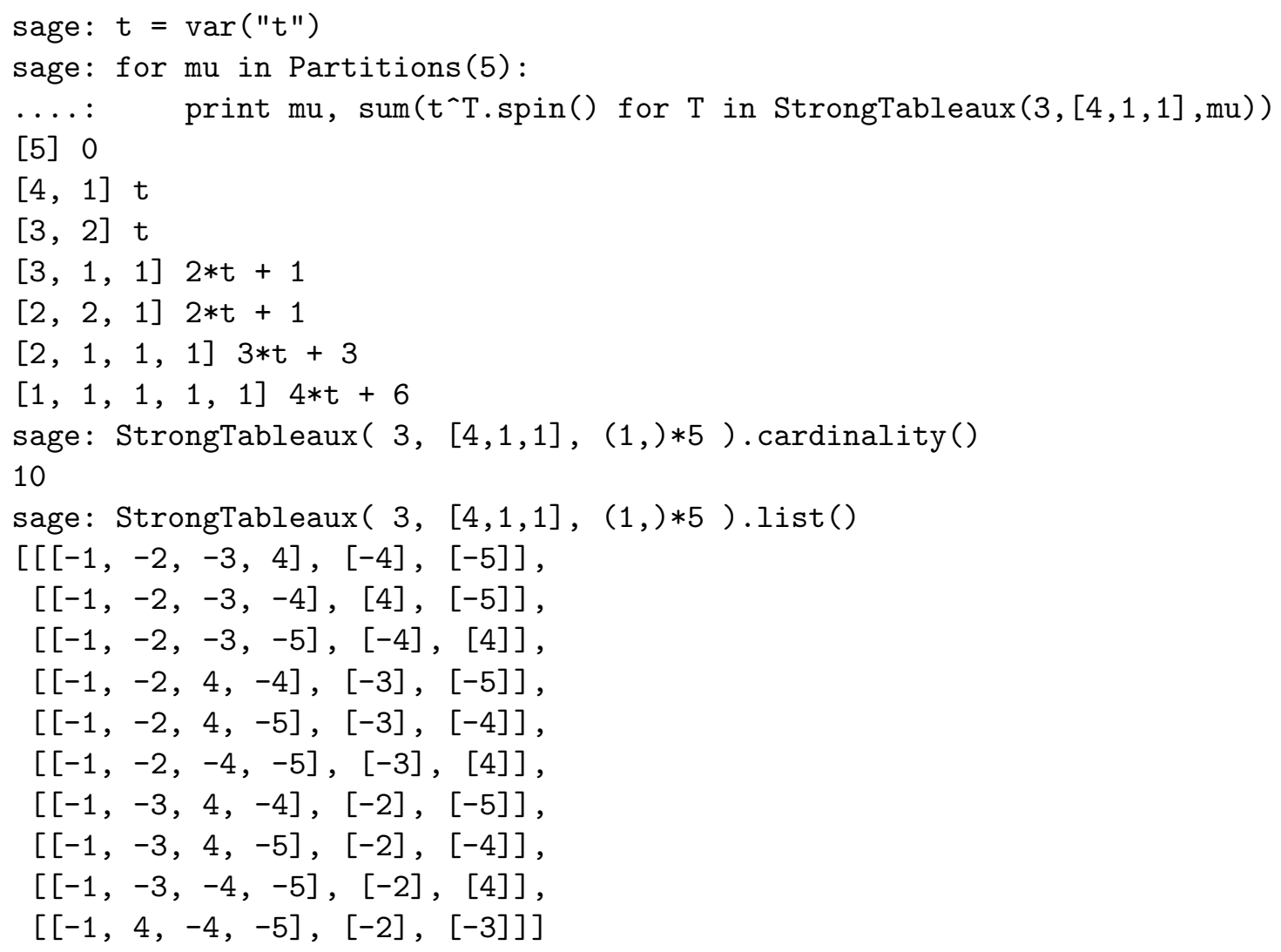

The definitions $A_{\lambda}^{(k)}[X ; t], \tilde{A}_{\lambda}^{(k)}[X ; t], s_{\lambda}^{(k)}[X ; t]$, and $\tilde{s}_{\lambda}^{(k)}[X ; t]$ of what are generically known as $k$-Schur functions have very different characters. Each connects to a different area of algebraic combinatorics and has its own benefits and detriments. The conjectured properties of $k$-Schur functions are sometimes clear from one definition, but difficult to prove for another. In the next section we will examine these properties and see why it would be very beneficial to resolve the following conjecture.

Conjecture 3.25. For $k>0$ and a $k$-bounded partition $\lambda$,

$$
s_{\lambda}^{(k)}[X ; t]=A_{\lambda}^{(k)}[X ; t]=\tilde{A}_{\lambda}^{(k)}[X ; t]=\tilde{s}_{\lambda}^{(k)}[X ; t] .
$$




\subsection{Notes on references}

The atom definition of the $k$-Schur functions was the first description and they were referred to by Alain Lascoux as potatoes ('les patates') since when copies of the atoms were identified on photocopies of the cyclage poset they could be circled to form tuber-like shapes (which we have endeavored to recreate in Example 3.1). The combinatorial definition uses the concept of katabolizable tableaux $[146,147]$ which generalizes the notion of cyclage which comes from [107]. The non-recursive definition of the charge statistic on tableaux also comes from [107].

It turns out that the atom definition is still roughly the easiest and fastest definition to implement on a computer. The implementation of the $k$-Schur functions in SAGE uses the definition of $A_{\lambda}^{(k)}[X ; t]$. For this reason, it is important to note that while Conjecture 3.10 has been checked up to degree $m=19$, Conjecture 3.25 has only been checked up to bounded partitions of $m=11$, but for $m>11$ there is currently no proof that these definitions are equivalent in general, even for $t=1$.

The operators $\mathbf{B}_{\lambda}$ from Equation (3.6) were defined by Shimozono and Zabrocki in [149] as a tool for understanding the generalized Kostka polynomials (also known as parabolic Kostka coefficients) that were studied by Shimozono and Weyman [148]. The combinatorial interpretation for the Schur expansion of a composition of these operators when the indexing partitions concatenate to a partition in terms of katabolizable tableaux is still an open problem. Certain cases of this are known (e.g. [144]) and its resolution would be helpful in attacking Conjecture 3.10. In [23] the Bernstein operators at $t=1$ are used to derive a recursion relation for the $k$-Schur functions which allow an easy expansion in the complete homogeneous basis.

In References $[96,97]$ the definition of the $k$-Schur functions at $t=1$ is taken to be the symmetric functions which satisfy the $k$-Pieri rule of Equation (2.19). Later Lam, Lapointe, Morse and Shimozono [81] showed that the $k$-Schur functions which satisfy the $k$-Pieri rule (2.19) are equivalent to Equation (3.16) at $t=1$, and Equation (3.16) is [81, Conjecture 9.11]. Assaf and Billey [6, Definition 3.2] have a slightly different statement of Equation (3.16) as a quasi-symmetric function expansion in the fundamental basis.

Recently, Dalal and Morse [35] have proven a second characterization of the $k$-Pieri at $t=1$ which leads to a very different sort of tableaux that enumerate $K_{\lambda \mu}^{(k)}$. They use these tableaux to provide an alternative combinatorial interpretation to Equation (1.38).

We note that the line between what is called a 'conjectured property' and what is called a 'definition' is sometimes a little blurry because we have provided several definitions of $k$ Schur functions which are conjectured to be equivalent. There are reasons to do this instead of taking one as definition and the rest as conjectured formulas; historically the $k$-Schur functions were presented in the literature this way. However, we note that there may be more definitions of the $k$-Schur functions than presented in this section. For instance, the $k$-shape poset presented in Section 7 and the representation theoretical definition which is briefly discussed in Section 5.5 are two other properties which may be taken as definitions, 
but are currently only conjectured to be equivalent to the definitions presented here.

\section{Properties of $k$-Schur functions and their duals}

In this section we list many of the properties of the $k$-Schur functions, both conjectured and proven. This section is mainly meant to be a statement of the 'current state of affairs' and is likely to change. There may not be a lot to say about each of these properties and instead we provide a proper reference for the statement of the conjecture or property, but we shall endeavor to add a few words about what needs to be proven in order to say why the property is true. The properties are each marked in the discussion with one of four headers $A^{(k)}, \tilde{A}^{(k)}, \tilde{s}^{(k)}, s^{(k)}$ to indicate comments on which of the four definitions (the $k$-atom definition of Section 3.1, the operator definition of Section 3.2, the weak tableaux definition of Section 3.3, and the strong tableaux definition of Section 3.4). Since $s_{\lambda}^{(k)}=\tilde{s}_{\lambda}^{(k)}$ at $t=1$ by [81, Theorem 4.11], we often group these two definitions together.

\section{1 $k$-Schur functions are Schur functions when $k \geq|\lambda|$ and when $t=0$}

Note that the stated property for $k \geq|\lambda|$ (resp. $t=0$ ) are two different statements. Nevertheless, it has been proven for all definitions of the $k$-Schur functions. It is known that $s_{\lambda}^{(k)}[X ; t]=s_{\lambda}$ plus other terms indexed by partitions $\mu$ which are strictly larger in dominance order, each with a positive power of $t$ as a coefficient.

$A^{(k)}$ : (proven, [92, Property 6]) When $k$ is larger than the largest hook of $\lambda$, then the only katabolizable tableau is the semi-standard tableau of shape $\lambda$ and weight $\lambda$. The charge of this tableau is 0 so it is clear that $A_{\lambda}^{(k)}[X ; t]=s_{\lambda}$. For $t=0$, we know that $A_{\lambda}^{(k)}[X ; 0]=s_{\lambda}$ because only the tableau of shape $\lambda$ and weight $\lambda$ has charge 0 in the atom tableaux.

$\tilde{A}^{(k)}$ : (proven, [93, Property 8]) This is slightly more complicated than the tableau definition but notice that $G_{\lambda}^{(k)}[X ; t]=s_{\lambda}$ if $k \geq|\lambda|$. The action of the operator $\mathbf{B}_{m}\left(s_{\lambda}\right)=$ $s_{\left(m, \lambda_{1}, \ldots, \lambda_{\ell(\lambda)}\right)}+$ other terms with first part which is larger than $m$ (and hence will be killed by the operator $\left.T_{m}^{(k)}\right)$. Also at $t=0$, the operator $\left.\mathbf{B}_{m}\right|_{t=0}=\mathbf{S}_{m}$ and $G_{\lambda}^{(k)}[X ; 0]=s_{\lambda}$.

$s^{(k)}$ : (proven, [96, Property 39]) One would need to trace through the definition of the strong order, but for $k \geq|\lambda|$, all marked strong tableaux will be standard tableaux and the markings of the marked strong tableaux are exactly the condition that these tableaux should be isomorphic to semi-standard tableaux. Because the heights of the ribbons are all 1 , the spins for all tableaux are 0. Therefore, $s_{\lambda}^{(k)}[X ; t]=\sum_{\mu} K_{\lambda \mu} m_{\mu}=s_{\lambda}$.

$\tilde{s}^{(k)}$ : (proven) When $k$ is large, weak $k$-tableaux are usual semi-standard tableaux and (3.13) turns into the definition of Schur functions. Similarly, $Q_{\lambda}^{\prime}[X ; 0]=s_{\lambda}$ which shows the second statement. 


\subsection{The $k$-Schur function is Schur positive}

In fact, a more refined conjecture to the statement that $k$-Schur functions are Schur positive, is that the $k$-Schur functions expand positively in the $(k+1)$-Schur functions. This property is usually referred to as ' $k$-branching' and is discussed in slightly more detail in Section 4.10 and again in Section 7. Repeated applications of the $k$-branching property yields a positive expansion of the $k$-Schur functions in the Schur functions. An explicit rule for the $k$ branching formula with a $t$ is conjectured in [82, Conjecture 1].

$A^{(k)}$ : (proven, [92, Property 7]) This property follows directly from the definition. Since $A_{\lambda}^{(k)}[X ; t]$ is a sum of Schur functions, one for each tableau in the set, this property is true by definition.

$\tilde{A}^{(k)}$ : (conjecture, [93, Equation (1.6)]) Equation (3.8) is an outstanding conjecture due to Shimozono and Weyman [148] that $H_{\lambda^{(*)}}[X ; t]$ is Schur positive whenever the concatenation of all of the partitions of $\lambda^{(*)}$ form a partition (which happens for all $k$-splits of partitions). Conjecture 3.10 is likely to follow from this conjecture and hence so would the property that $\tilde{A}_{\lambda}^{(k)}[X ; t]$ is Schur positive.

$s^{(k)}$ : (for arbitrary $t$ it follows from [82, Conjecture 1], for $t=1$ follows from [82, Theorem 2]) In Section 7 we will give a precise conjecture of how $s_{\lambda}^{(k)}[X ; t]$ expands positively in $s_{\lambda}^{(k+1)}[X ; t]$. If we iteratively apply that rule, it must be that $s_{\lambda}^{(k)}[X ; t]$ expands positively in the limit as $k$ increases, and when $k \geq \lambda_{1}+\ell(\lambda)-1$ then $s_{\lambda}^{(k)}[X ; t]=s_{\lambda}[X]$. In addition, some conjectures of [81] were proven in [13], in particular that "skew shaped" strong Schur functions are symmetric.

Assaf and Billey [6] convert the monomial expansion of $k$-Schur functions in terms of strong marked tableaux into a quasi-symmetric function expansion. They conjectured that a dual equivalence graph structure can be placed on strong tableaux and using Assaf's earlier work $[4,5]$ this structure can in theory be used to show that the $s_{\lambda}^{(k)}[X ; t]$ are Schur positive as long as a computer check on a finite number of elements is completed. Billey informs us that this calculation has a current estimated running time which is quite long and has not yet been completed.

$\tilde{s}^{(k)}$ : (conjecture, proven for $t=1$ ) Since $s_{\lambda}^{(k)}=\tilde{s}_{\lambda}^{(k)}$ for $t=1$, this property is true by [82, Theorem 2]. A promising approach for generic $t$ is underway; by duality, the positivity of $\tilde{s}_{\lambda}^{(k)}[X ; t]$ in terms of $\tilde{s}_{\mu}^{(k+1)}[X ; t]$ 's follows from the positivity of dual $k+1$ Schur functions into dual $k$-Schur functions. This, in turn, would follow by showing that there is a compatibility between $k$-charge and the weak bijection introduced in [82]. A partial solution has been given in [99], where the compatibility is shown for standard $k$ tableaux. 


\subsection{At $t=1$, the $k$-Schur functions satisfy the $k$-Pieri rule}

In Sections 2.2 and 2.4 we discussed the $k$-Pieri rule at $t=1$. It states that for $1 \leq r \leq k$, $h_{r} s_{\lambda}^{(k)}[X ; 1]=\sum_{\mu} s_{\mu}^{(k)}[X ; 1]$ where the sum is over all partitions $\mu$ such that $\mu \vdash|\lambda|+r$, $\mathfrak{c}(\lambda) \subseteq \mathfrak{c}(\mu)$ and $\mathfrak{c}(\mu) / \mathfrak{c}(\lambda)$ is a weak horizontal strip.

$A^{(k)}$ : (conjecture [92, Conjecture 41]) The tableaux operations for the definition of the $k$ atoms are somewhat unusual and more work needs to be done to understand many of their properties. Combinatorially, the $k$-Pieri rule is most clearly stated in terms of $(k+1)$-cores, the connection between $k$-atoms and $(k+1)$-cores is not clear in this definition.

$\tilde{A}^{(k)}$ : (conjecture [93, Conjecture 21]) In order to prove this property it seems as though it would be necessary to understand the commutation relationship between multiplication by a symmetric function $h_{r}$ and the operators $T_{i}$ of Equation (3.9) when $t=1$.

$\tilde{s}^{(k)}$ : (proven [96, Theorem 29]) This property follows by showing that the number of weak $k$-tableaux of weight $\alpha$ equals the number of $k$-tableaux of weight $\beta$, for $\beta$ any rearrangement of the parts of $\alpha$.

$s^{(k)}$ : (proven [81, Theorem 4.11]) It was proven in [81] that $s_{\lambda}^{(k)}=\tilde{s}_{\lambda}^{(k)}$ when $t=1$. The result then follows from the Pieri rule on $\tilde{s}_{\lambda}^{(k)}$ from [96].

It is conjectured that the $k$-Schur functions also satisfy a $t$-analogue of the $k$-Pieri rule Equation (2.19) where the operator $\mathbf{B}_{m}$ takes the role of multiplication by $h_{m}$ (and reduces as such when $t=1$ ). Recall that Zabrocki [158] determined the action of $\mathbf{B}_{m}$ on Schur functions in his thesis and he gave a new proof for the charge formulation of Hall-Littlewood polynomials $Q_{\lambda}[X ; t]$.

Given a $(k+1)$-core $\lambda$ with $\lambda_{1}=m$, let $\rho$ be the unique partition whose first part is $m$ and where $\rho / \lambda$ is a horizontal strip of size $m$ (that is, $\rho$ is obtained by adding a cell to the top of each column of $\lambda$ ). For $\mathfrak{p}(\lambda)_{1} \leq r \leq k$, Maria-Elena Pinto conjectured that

$$
\mathbf{B}_{r} s_{\lambda}^{(k)}[X ; t]=\sum_{\mu / \lambda \text { weak } r \text {-strip }} t^{a(\mu)} s_{\mu}^{(k)}[X ; t],
$$

where $a(\mu)$ is the number of cells of $\rho / \lambda$ whose residue does not label a cell of $\mu / \lambda$ (see also [36] for a different conjectured formula using strong order chains in $\tilde{A}^{k}$ ).

Example 4.1. Let $k=5$ and consider $\lambda=(4,2,1,1)$, so that $\mathfrak{p}(\lambda)=(3,2,1,1)$.

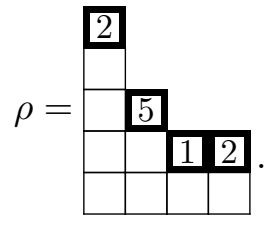


Possible horizontal weak 3-strips are
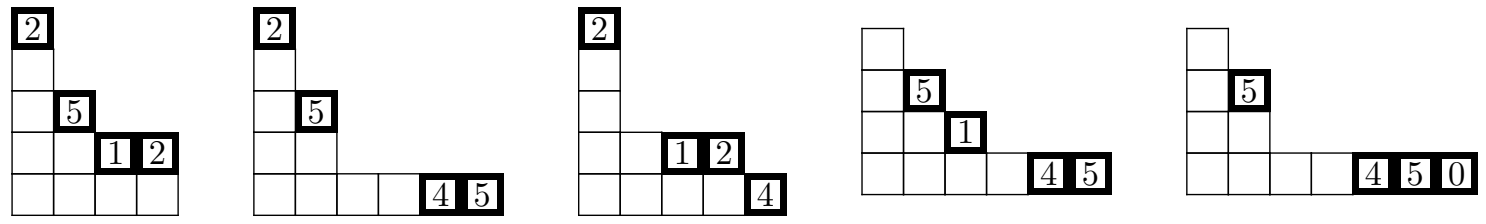

and their respective powers are $t^{0}, t^{1}$ (the cell of residue 1 in $\rho$ ), $t^{1}$ (the cell of residue 5 in $\rho$ ), $t^{2}$ (the two cells of residue 2 in $\rho$ ) and $t^{3}$ (the cell of residue 1 and the 2 cells of residue 2 in $\rho$ ). This gives

$$
\mathbf{B}_{3} s_{4211}^{(5)}[X ; t]=s_{44211}^{(5)}[X ; t]+t s_{62211}^{(5)}[X ; t]+t s_{54111}^{(5)}[X ; t]+t^{2} s_{6321}^{(5)}[X ; t]+t^{3} s_{7221}^{(5)}[X ; t] .
$$

As with the action of $\mathbf{B}_{m}$ on a Schur functions, if $m<\lambda_{1}$, the $k$-Schur expansion of $\mathbf{B}_{m}\left(s_{\lambda}^{(k)}[X ; t]\right)$ has negative terms. Currently, there is no conjecture describing these terms that cancel when $t=1$.

Sage Example 4.2. Here we demonstrate the action of the $\mathbf{B}_{m}$ operator on the $k$-Schur function basis. For $k \geq m \geq \lambda_{1}-1$ this is conjectured to expand positively in the $k$-Schur basis.

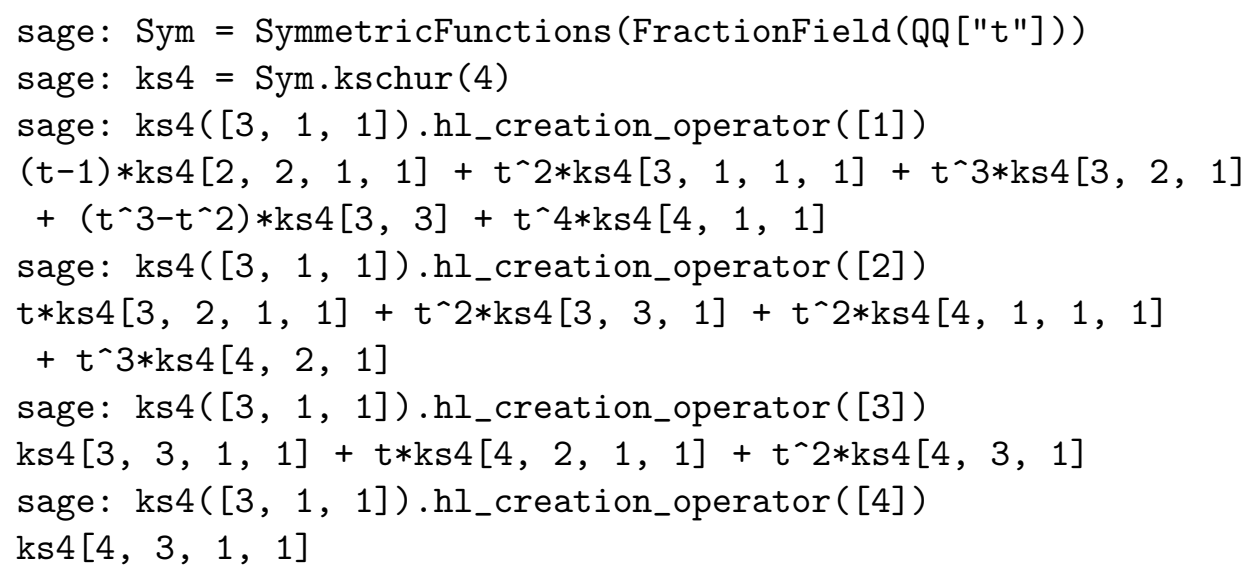

\section{$4.4 \quad k$-conjugation}

The $\omega$-involution sending $s_{\lambda}$ to $s_{\lambda^{\prime}}$ acts simply on $k$-Schur functions as well. It was conjectured in $[92,93]$ that, for some non-negative power of $t$,

$$
\omega\left(s_{\lambda}^{(k)}[X ; t]\right)=t^{d} s_{\lambda^{\omega_{k}}}^{(k)}[X ; 1 / t]
$$

where $\lambda^{\tilde{\omega}_{k}}$ is a $k$-bounded partition depending on $\lambda$ and $k$. Later, it was shown in [95] that $\lambda^{\tilde{\omega}_{k}}=\lambda^{\omega_{k}}$ corresponds to usual conjugation in the $k+1$-core framework (see Definition 1.19). One consequence of $k$-branching [82] is that the power of $d$ counts cells of $\mathfrak{c}(\lambda)$ with hook greater than $k$ (see also [32]). 
$A^{(k)}$ : (conjecture [92, Conjecture 36]) Using the definition of $k$-Schur functions in terms of tableaux atoms, there is definitely an orientation in the definition of katabolism that is not compatible with the notion of conjugation of the tableau. This is because the first step of the definition involves a split of the reading word of the tableau which involves reading the rows, and it really is not clear how this would carry to the columns.

One possible approach to the tableaux definition would be to show that there is a bijection between the tableaux in the atoms $\mathbb{A}_{\lambda}^{(k)}$ and $\mathbb{A}_{\lambda \omega_{k}}^{(k)}$. Since the $k$-conjugation is most easily expressed in terms of $(k+1)$-cores, it seems that some connection between atoms and $(k+1)$-cores will need to be found.

$\tilde{A}^{(k)}$ : (conjecture [93, Conjecture 40], at $t=1$ this is [93, Conjecture 19]) Since the algebraic definition depends on the operation of $k$-split, it is not clear how the action of $\omega$ interacts with the individual operators. There is some hope that some algebraic tools will be developed to resolve this conjecture by looking at the expansion in terms of $s_{\lambda}[X /(1-t)]$ since for an element $f[X ; t] \in \Lambda_{(n)}^{t}$ it is at least known that $\omega(f[X ; 1 / t]) \in \Lambda_{(n)}^{t}$.

$s^{(k)}, \tilde{s}^{(k)}$ : (conjecture, for $t=1$ this is [96, Theorem 38]) At $t=1$ this property follows because of [96, Theorem 33], where a formula for the product of $e_{r}$ and a $k$-Schur function is given as

$$
e_{r} s_{\lambda}^{(k)}=\sum_{\mu} s_{\mu}^{(k)}
$$

where the sum is over all $k$-bounded partitions $\mu$ of size $|\lambda|+r$ such that $\mu / \lambda$ is a vertical strip and $\mu^{\omega_{k}} / \lambda^{\omega_{k}}$ a horizontal strip.

Sage Example 4.3. Let us check an example of equation (4.2) by a calculation in SAGE. In order to invert the parameter $t$, we must first expand the $k$-Schur function in a basis which is independent of the parameter $t$. In fact, if we apply the involution $\omega$ alone, the function no longer lies in the space spanned by the $k$-Schur functions. However, if we apply $\omega$ and invert the parameter, then it does belong to the right space.

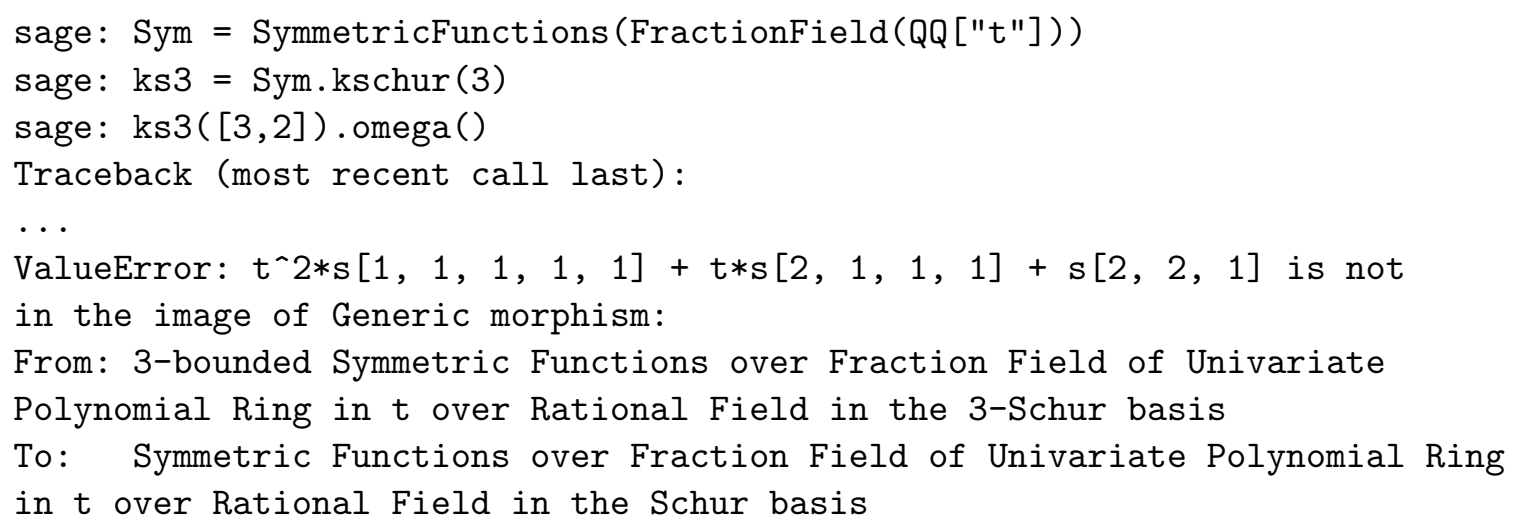




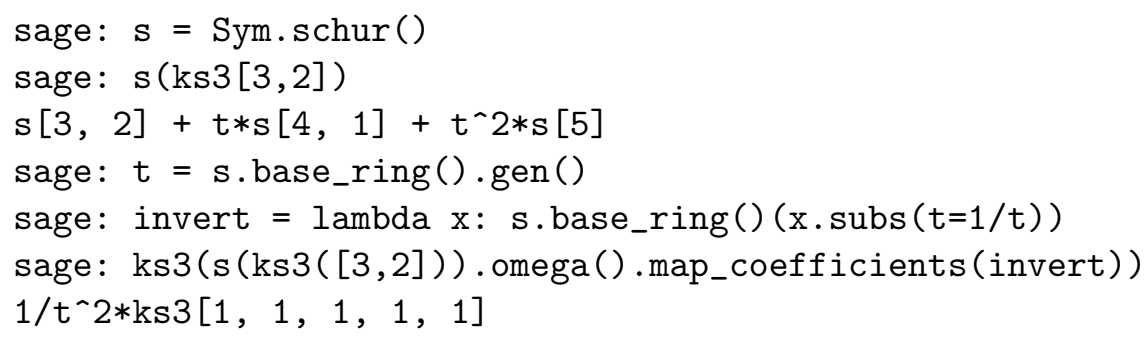

In fact, there is a short-cut for the last computation in SAGE by simply asking

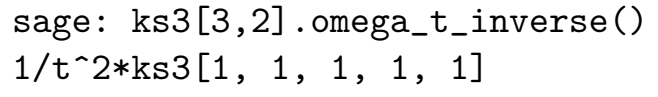

\subsection{The $k$-Schur functions form a basis for $\Lambda_{(k)}^{t}$}

Recall from Equation (3.5) that the definition of $\Lambda_{(k)}^{t}$ is the linear span over $\mathbb{Q}(q, t)$ of the symmetric functions $H_{\lambda}[X ; q, t]$ (or $s_{\lambda}[X /(1-t)]$ or $\left.Q_{\lambda}^{\prime}[X ; t]\right)$ over all partitions $\lambda$ with $\lambda_{1} \leq k$. For each of our definitions, it is not necessarily clear that the $k$-Schur functions even lie in $\Lambda_{(k)}^{t}$. However, if they do and if they are linearly independent, they will form a basis since they are also indexed by $k$-bounded partitions.

$A^{(k)}$ : (conjecture [92, Conjecture 8]) The atoms are known to be linearly independent [92, Property 7] since they are triangular with respect to the Schur functions. Nevertheless, it remains a conjecture that they are elements of $\Lambda_{(k)}^{t}$; their combinatorial definition does not give a direct connection with the known bases of $\Lambda_{(k)}^{t}$. It seems as though the most likely means of proving this conjecture is to show Conjecture 3.10, otherwise there is no obvious connection with the spanning elements which define $\Lambda_{(k)}^{t}$.

$\tilde{A}^{(k)}$ : (proven [93, Theorem 33]) This result is non-trivial because it is not easy to demonstrate that the elements $G_{\lambda}^{(k)}[X ; t]$ form a basis of $\Lambda_{(k)}^{t}$.

$s^{(k)}$ : (conjecture, discussion of this definition is in [81, Section 9.3] but this particular property is not directly addressed; at $t=1$ this is [96, Property 27]) In reference [96] the $k$-Schur functions $s_{\lambda}^{(k)}$ are defined as the basis which satisfies the $k$-Pieri rule of (2.19) and from that definition it is clear that $s_{\lambda}^{(k)} \in \Lambda_{(k)}$. The fact that they form a basis follows from a unitriangularity relation with the basis $\left\{h_{\lambda}: \lambda_{1} \leq k\right\}$ that follows from the $k$-Pieri rule.

$\tilde{s}^{(k)}$ : (proven [35]) Since $Q_{\lambda}^{\prime}[X ; t]$ forms a basis of $\Lambda_{(k)}^{t}$ and the matrix $K_{\lambda \mu}^{(k)}(t)$ is invertible, $\tilde{s}_{\lambda}^{(k)}[X ; t]$ also forms a basis of $\Lambda_{(k)}^{t}$. 


\subsection{The $k$-rectangle property}

A remarkable property of the $k$-Schur functions is that it is trivial to multiply any $s_{\lambda}^{(k)}$ by a $k$-Schur function indexed by a $k$-rectangle - any partition of the form $\left(\ell^{k-\ell+1}\right)$. Precisely, for any $k$-bounded partition $\lambda$ and any integer $1 \leq \ell \leq k$,

$$
s_{\ell^{k+1-\ell}} s_{\lambda}^{(k)}=s_{\lambda \cup \ell^{k+1-\ell}}^{(k)},
$$

where $\lambda \cup \nu$ depicts the partition obtained by putting the parts of $\lambda$ and $\nu$ into nonincreasing order. In fact, this property has a generic $t$ analog in which the Schur function $s_{\ell^{k+1-\ell}}$ is replaced by the operator $\mathbf{B}_{\ell^{k+1-\ell}}$ defined in Equation (3.6). Then, given $\lambda$ and an integer $1 \leq \ell \leq k$,

$$
\mathbf{B}_{\left(\ell^{k-\ell+1}\right)} s_{\lambda}^{(k)}[X ; t]=t^{|\mu|-\ell(\mu) \ell} s_{\left(\ell^{k-\ell+1}\right) \cup \lambda}^{(k)}[X ; t],
$$

where $\lambda=(\mu, \nu)$ with $\mu_{\ell(\mu)}>\ell \geq \nu_{1}$. A by-product of this result is that any $k$-Schur function can be obtained by $k$-rectangle translation of elements in a distinguished set of $k$ ! $k$-Schur functions. These $k$ ! elements are those indexed by irreducible partitions -partitions with at most $k-r$ parts of size $r$, for $1 \leq r \leq k$. For any $k$-bounded partition $\nu$, up to a $t$-factor,

$$
s_{\nu}^{(k)}[X ; t]=\mathbf{B}_{R_{1}} \mathbf{B}_{R_{2}} \cdots \mathbf{B}_{R_{d}} s_{\lambda}^{(k)}[X ; t],
$$

where $\lambda$ is the irreducible partition obtained by removing $k$-rectangles $R_{1}, \ldots, R_{d}$ from $\nu$.

$A^{(k)}$ : (conjecture [92, Conjecture 21]) This is not known and there are no obvious techniques to be tried. One caveat of the atom definition is that the $t=1$ case does not simplify things; the result is also unknown when $t=1$.

$\tilde{A}^{(k)}$ : (proven [94, Theorem 26]) This is shown by developing properties of the $\mathbf{B}_{\lambda}$ operators and the commutation relations with the operator $T_{m}^{(k)}$.

$s^{(k)}, \tilde{s}^{(k)}$ : (conjecture, but proven for $t=1$ in [96, Theorem 40]) When $t=1$, the operator $\mathbf{B}_{R}$ reduces to multiplication by $s_{R}$ and it was shown that the linear operation of adding a $k$-rectangle to the index of a $k$-Schur function commutes with the Pieri rule from Equation (2.19).

\subsection{When $t=1$, the product of $k$-Schur functions is $k$-Schur positive}

Note that $\Lambda_{(k)}^{t}$ is not an algebra and the product of two arbitrary $k$-Schur functions does not remain in the space. However, when $t=1$ we have $\Lambda_{(k)}^{t=1}=\Lambda_{(k)}$ and as discussed in Section 2.2, the space defined in Equation (2.8) is closed under multiplication. The structure coefficients $c_{\lambda \mu}^{\nu(k)}$ defined by

$$
s_{\lambda}^{(k)} s_{\mu}^{(k)}=\sum_{\nu} c_{\lambda \mu}^{\nu(k)} s_{\nu}^{(k)}
$$


are non-negative integer coefficients. Recall from Section 2.5 that these are now called $k$ Littlewood-Richardson coefficients and they are a family of constants that includes GromovWitten invariants for complete flag varieties, WZW-fusion coefficients, and the structure constants of Schubert polynomials.

$A^{(k)}$ : (conjecture [92, Conjecture 39]) Note that the $k$-Pieri rule is a special case of computing the product of two $k$-Schur functions. As discussed in Section 4.3, the techniques to work with atoms have yet to be developed and even this 'simple' case remains unproven. However, the atom definition is likely to be useful in gaining insight into the combinatorial nature of the structure coefficients.

$\tilde{A}^{(k)}:$ (conjecture [93, Conjecture 20]) In this case we have again that the $k$-Pieri rule at $t=1$ is a conjecture. It would be sufficient to show that $s_{\lambda}^{(k)}[X ; 1]=\tilde{A}_{\lambda}^{(k)}[X ; 1]$ since the $k$-Pieri rule characterizes $s_{\lambda}^{(k)}[X ; 1]$ and hence the result is known in this case. It seems that the algebraic definition using operators might be helpful finding a $t$-analogue of the coefficients $c_{\lambda \mu}^{\nu(k)}$.

$s^{(k)}, \tilde{s}^{(k)}$ : (proven [79, Corollary 8.2]) Lam [79] proved that the $s_{\lambda}^{(k)}[X ; 1]$ are isomorphic to the Schubert basis for the homology of the affine Grassmannian. From geometric considerations, it follows that the structure coefficients $c_{\lambda \mu}^{\nu(k)}$ enumerate certain curves in a finite flag variety.

\subsection{Positively closed under coproduct}

The space $\Lambda_{(k)}^{t}$ is not an algebra, but since it is linearly spanned by the elements $s_{\lambda}[X /(1-t)]$ for $\lambda_{1} \leq k$, it is a coalgebra under the coproduct defined by

$$
\Delta\left(s_{\lambda}[X /(1-t)]\right)=\sum_{\mu, \nu} c_{\mu \nu}^{\lambda} s_{\mu}[X /(1-t)] s_{\nu}[Y /(1-t)]
$$

(where the $c_{\mu \nu}^{\lambda}$ are the Littlewood-Richardson coefficients). Compare this also with the coproduct (1.28) on $\Lambda$. Since if $\lambda$ is $k$-bounded then all of the terms $\mu, \nu$ which appear in this expansion will also be $k$-bounded, it is conjectured that if the coefficients $C_{\mu \nu}^{\lambda}(t)$ are defined as the coefficients in the expansion

$$
\Delta\left(s_{\lambda}^{(k)}[X ; t]\right)=\sum_{\mu, \nu} C_{\mu \nu}^{\lambda(k)}(t) s_{\mu}^{(k)}[X ; t] s_{\nu}^{(k)}[Y ; t]
$$

then the $C_{\mu \nu}^{\lambda}(t)$ are polynomials in $t$ with non-negative integer coefficients.

$A^{(k)}:$ (conjecture [92, Conjecture 17])

$\tilde{A}^{(k)}:($ conjecture $[93$, Conjecture 41]) 
$s^{(k)}$ : (conjecture, proven for $t=1$ in [79, Corollary 8.1]) Because of the duality of the elements $\tilde{F}_{\mu}^{(k)}$ to the elements $s_{\lambda}^{(k)}$, it follows that at $t=1$, the basis $\tilde{F}_{\lambda}^{(k)}$ of the space $\Lambda^{(k)}$ multiplies as

$$
\tilde{F}_{\mu}^{(k)} \tilde{F}_{\nu}^{(k)}=\sum_{\lambda} C_{\mu \nu}^{\lambda(k)} \tilde{F}_{\lambda}^{(k)}
$$

where $C_{\mu \nu}^{\lambda(k)}=C_{\mu \nu}^{\lambda(k)}(1)$.

Although we can say little about the $A^{(k)}$ and $\tilde{A}^{(k)}$ cases, we can determine from the definition of $s_{\lambda}^{(k)}[X ; t]$ that

$$
s_{\lambda}^{(k)}[X+z ; t]=\sum_{r \geq 0} z^{r} h_{r}^{\perp} s_{\lambda}^{(k)}[X ; t]=\sum_{r \geq 0} z^{r} C_{\mu(r)}^{\lambda(k)}(t) s_{\mu}^{(k)}[X ; t] .
$$

In this special case the coefficients are

$$
C_{\mu(r)}^{\lambda(k)}(t)=\sum_{\kappa^{(*)}, c_{*}} t^{\operatorname{spin}\left(\kappa^{(*)}, c_{*}\right)}
$$

with the sum is over all strong marked horizontal strips from $\mathfrak{c}(\mu)$ to $\mathfrak{c}(\lambda)$. That is, the sum runs over $\kappa^{(*)}, c_{*}$ which are $(k+1)$-core tableaux of the form

$$
\kappa^{(0)}=\mathfrak{c}(\lambda) \Rightarrow_{k} \kappa^{(1)} \Rightarrow_{k} \kappa^{(2)} \Rightarrow_{k} \cdots \Rightarrow_{k} \kappa^{(r)}=\mathfrak{c}(\mu)
$$

and markings $c_{1}<c_{2}<\cdots<c_{r}$ where $c_{i}$ is the content of the lower right hand cell of one of the ribbons of $\kappa^{(i)} / \kappa^{(i-1)}$ and where $\operatorname{spin}\left(\kappa^{(*)}, c_{*}\right)$ is defined, as before, as the sum of the spins of the strong marked ribbons $\left(\kappa^{(i)} / \kappa^{(i-1)}, c_{i}\right)$.

$\tilde{s}^{(k)}$ : (conjecture) For $t=1, s_{\lambda}^{(k)}=\tilde{s}_{\lambda}^{(k)}$ and hence also follows from [79, Corollary 8.1].

Sage Example 4.4. Here is a calculation in SAGE where we observe that the coefficients that appear in an example of (4.9) are polynomials in $\mathbb{N}[t]$.

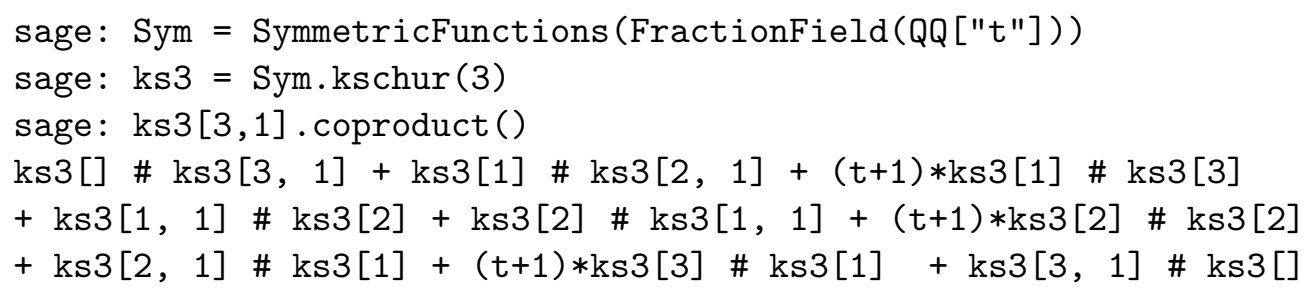

\subsection{The product of a $k$-Schur and $\ell$-Schur function is $(k+\ell)$-Schur positive}

Recall that one characterization of $\Lambda_{(k)}^{t}$ is that it is the linear span of $\left\{s_{\lambda}[X /(1-t)]\right\}_{\lambda_{1} \leq k}$. Now if we know that $f \in \Lambda_{(k)}^{t}$ and $g \in \Lambda_{(\ell)}^{t}$ then we know by the Littlewood-Richardson rule that $f g$ will be in the linear span of $\left\{s_{\lambda}[X /(1-t)]\right\}_{\lambda_{1} \leq k+\ell}$. By the discussion in Section 4.5, 
it is not even clear that the products $s_{\lambda}^{(k)}[X ; t] s_{\mu}^{(\ell)}[X ; t]$ or $A_{\lambda}^{(k)}[X ; t] A_{\mu}^{(\ell)}[X ; t]$ will be in the space $\Lambda_{(k+\ell)}^{t}$, but it has been proven that $\tilde{A}_{\lambda}^{(k)}[X ; t] \tilde{A}_{\mu}^{(\ell)}[X ; t]$ and $\tilde{s}_{\lambda}^{(k)}[X ; t] \tilde{s}_{\mu}^{(\ell)}[X ; t]$ is an element of $\Lambda_{(k+\ell)}^{t}$.

Given that the product of a $k$-Schur function and an $\ell$-Schur function is in the linear span of $\Lambda_{(k+\ell)}^{t}$, it is natural to conjecture that the resulting product will be $(k+\ell)$-Schur positive. We do not know of an attribution for this conjecture but it seems to have been passed around in discussions and talks on the subject.

Sage Example 4.5. We demonstrate an example of this conjecture in SAGE by showing that the product of a 3-Schur function and a 2-Schur function expands positively in terms of 5-Schur functions.

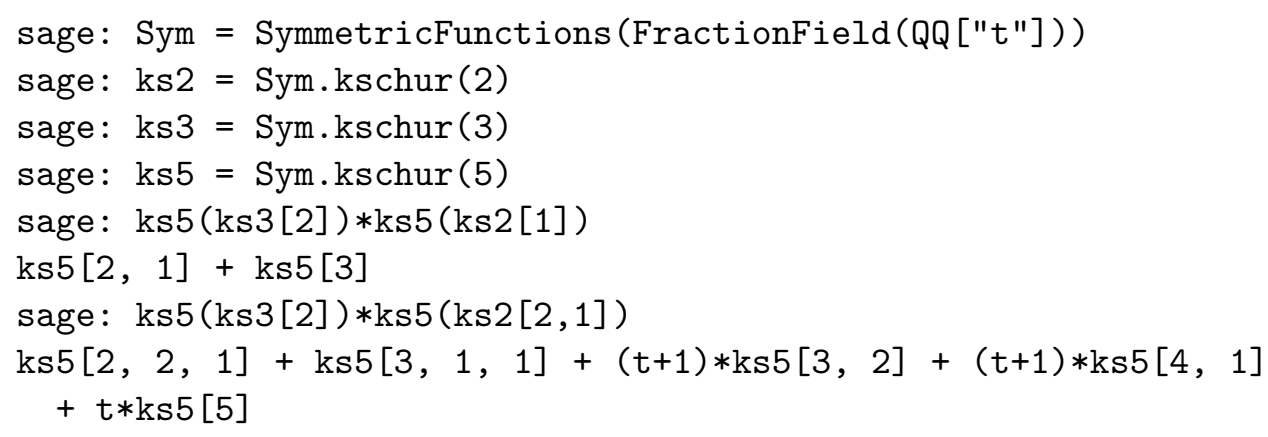

\subsection{Branching property from $k$ to $k+1$}

One of the properties that is easy to observe when conjecturing the existence of atoms is that the atoms seem to split into smaller pieces as $k$ increases. In the limit (when $k \geq|\lambda|$ ), we know that $s_{\lambda}^{(k)}[X ; t]=s_{\lambda}$ (see Section 4.1). Although it is clear that $\Lambda_{(k)}^{t} \subseteq \Lambda_{(k+1)}^{t}$, it is not easy to prove this branching property.

One reason in particular that this is a difficult property to understand is that both the definition of $A_{\lambda}^{(k)}$ and $\tilde{A}_{\lambda}^{(k)}$ involve the operation of the $k$-split, one in the katabolism procedure, and the other in the $k$-split basis $G_{\lambda}^{(k)}[X ; t]$. In theory the $k$-split of a partition $\lambda$ can be very different than the $(k+1)$-split of the same partition (e.g. consider the 4 and 5 split of $(4,4,4,3,3,3,2,2,1,1)$ which are $((4),(4),(4),(3,3),(3,2),(2,1,1))$ and $((4,4),(4,3),(3,3,2),(2,1,1))$ respectively). A priori we would not expect to see that $A_{\lambda}^{(k)}[X ; t]$ expands positively in $A^{(k+1)}[X ; t]$ or $\tilde{A}^{(k)}[X ; t]$ expands positively in $\tilde{A}_{\lambda}^{(k+1)}[X ; t]$. However this property was one that was used to conjecture/compute the $k$-atoms before there was a first formal definition.

$s^{(k)}$ : (proven for $t=1$ in [82], [82, Conjecture 3] is combinatorial formula) At $t=1$, the proof of the $k \rightarrow k+1$ branching for $s_{\lambda}^{(k)}[X ; t]$ follows from the study [82] of a poset on particular partitions called $k$-shapes. In Section 7 we will give some details on the 
combinatorics behind these results and state the explicit combinatorial formula for this rule in Theorem 7.7. In short, [82] proves that $s_{\lambda}^{(k)}$ expands positively in the elements $s_{\lambda}^{(k+1)}$ and gives a conjecture for the expansion of $s_{\lambda}^{(k)}[X ; t]$ in terms of elements of the form $s_{\lambda}^{(k+1)}[X ; t]$. For generic $t$, the same paper gives a conjecture formula Conjecture 7.8 and discusses the additional properties needed for the result to hold in general. Some progress has been made in this direction in [99].

$\tilde{s}^{(k)}$ : (conjecture) At $t=1, s_{\lambda}^{(k)}=\tilde{s}_{\lambda}^{(k)}$, hence the result in this case also follows from refrence [82].

Sage Example 4.6. Here are some examples confirming the branching conjecture (for the implementation in SAGE):

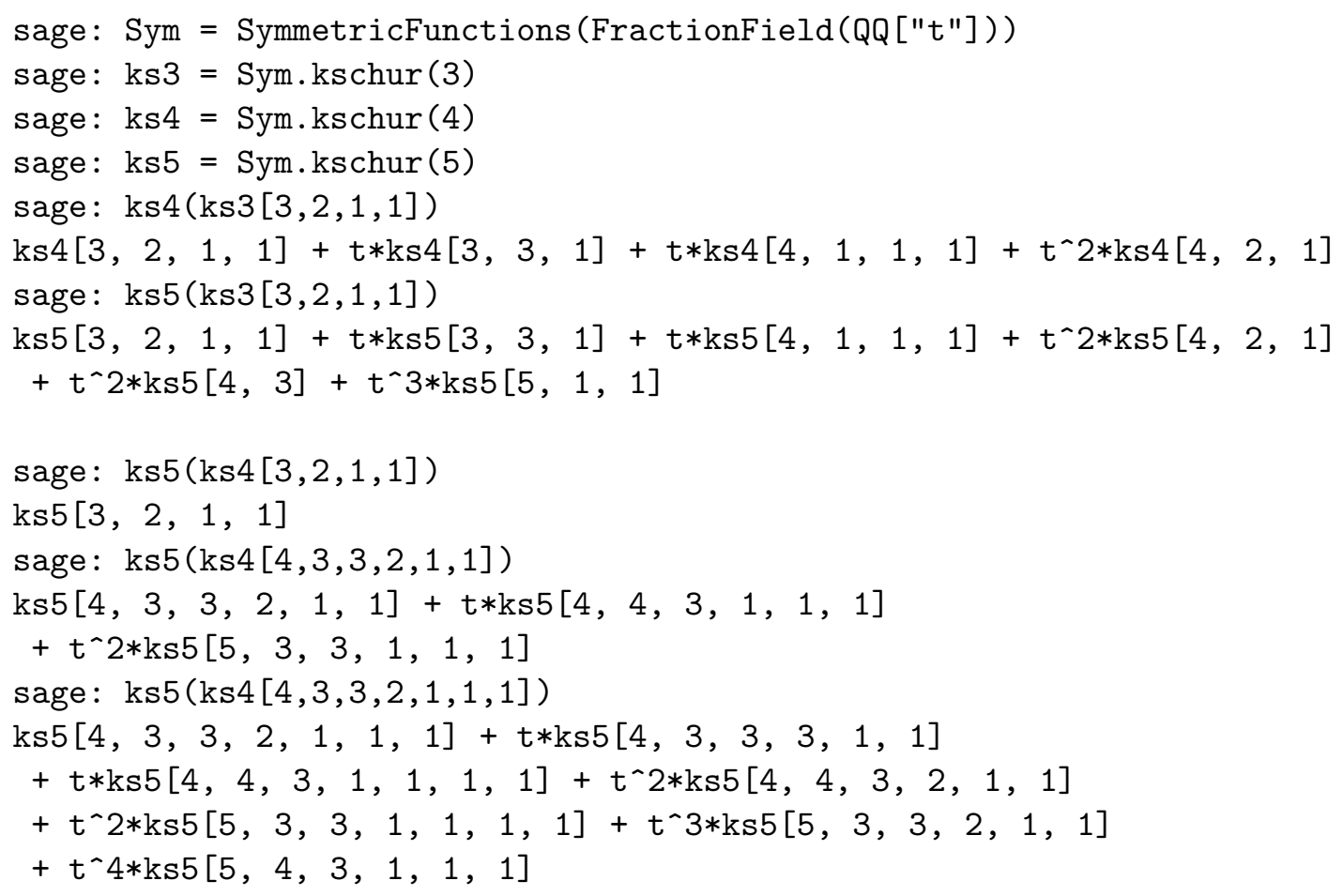

\section{$4.11 k$-Schur positivity of Macdonald symmetric functions}

Even equipped with all these definitions, it has yet to be understood why the Macdonald polynomials expand positively in terms of $k$-Schur functions. Recall from (1.39) that, for any $k$-bounded partition $\mu$, the coefficients in

$$
H_{\mu}[X ; q, t]=\sum_{\lambda: \lambda_{1} \leq k} K_{\lambda \mu}^{(k)}(q, t) s_{\lambda}^{(k)}[X ; t]
$$


are conjectured to be polynomials in $q$ and $t$ with non-negative integer coefficients. An ideal solution to this problem would be to find statistics $a_{\mu}^{(k)}$ and $b_{\mu}^{(k)}$ on weak tableaux such that

$$
K_{\lambda \mu}^{(k)}(q, t)=\sum_{T} q^{a_{\mu}^{(k)}(T)} t^{b_{\mu}^{(k)}(T)}
$$

where the sum is over all standard weak tableaux of shape $\lambda$. Section 3.3 discusses partial progress in this direction where such a solution is given for the cases $K_{\lambda \mu}^{(k)}(1,1)=K_{\lambda 1|\mu|}^{(k)}$ and $K_{\lambda \mu}^{(k)}(0,1)=K_{\lambda \mu}^{(k)}$.

$A^{(k)}$ : (conjecture [92, Conjecture 8]) This conjecture was the original motivation for studying $k$-Schur functions; it was a promising attack on a combinatorial interpretation of the Macdonald-Kostka coefficients especially when coupled with the conjecture [92, Eq. (1.15)] that $K_{\lambda \mu}(q, t)-K_{\lambda \mu}^{(k)}(q, t)$ is in $\mathbb{N}[q, t]$. However, a clear combinatorial interpretation of $K_{\lambda \mu}(q, t)$ remains elusive as does even the positivity of the polynomials $K_{\lambda \mu}^{(k)}(q, t)$.

$\tilde{A}^{(k)}$ : (conjecture [93, Eq. (1.7)]) A preliminary attack of the $k=2$ case of this conjecture was considered in [91] and [159] although the complete formulation of the conjecture had not been yet made. Lapointe and Morse together with Lascoux developed the ideas further into $k$-atoms. Even without knowledge of this conjecture, the latter reference also refers to collections of tableaux as 'atoms' and some of the symmetric functions defined there were actually the 2 -atoms.

$s^{(k)}$ : (conjecture [95, Eq. (11.6)]) It was because of the characterization of the $k$-Schur functions as the basis that satisfies the $k$-Pieri rule of Equation (2.19) that there is a combinatorial interpretation for $K_{\lambda \mu}^{(k)}(1,1)$ in terms of weak tableaux.

$\tilde{s}^{(k)}$ : (conjecture) This is a conjecture, but for $q=0, H_{\mu}[X ; 0, t]=Q_{\mu}^{\prime}[X ; t]$ and the definition [36] of $\tilde{s}_{\lambda}^{(k)}$ yields that $K_{\lambda \mu}^{(k)}(0, t)=K_{\lambda \mu}^{(k)}(t)$.

Sage Example 4.7. Here are some of the $k$-analogues of the $(q, t)$-Macdonald-Kostka coefficients computed in SAGE:

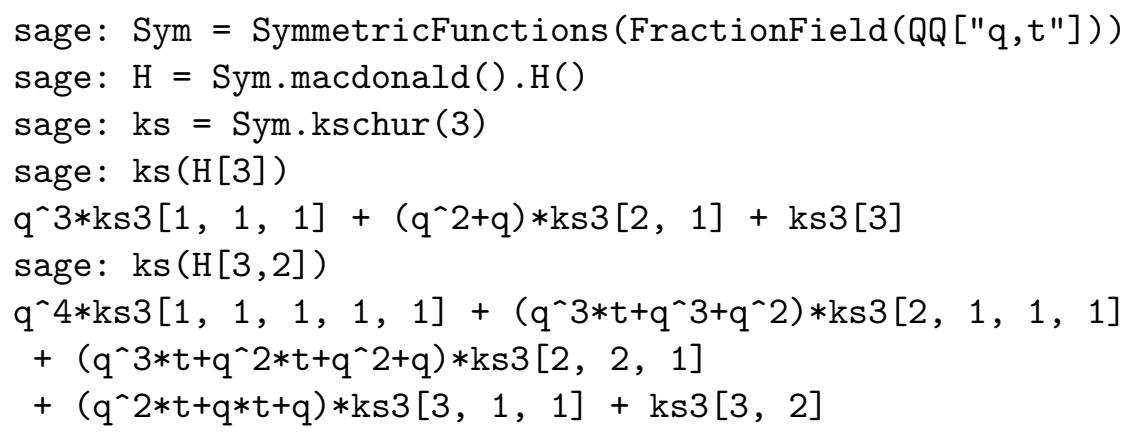




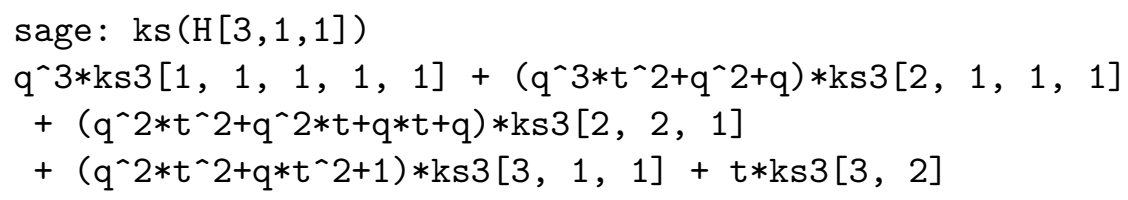

\section{Directions of research and open problems}

In this section we consider further directions of $k$-Schur research, some in their early stages.

\subsection{A $k$-Murnaghan-Nakayama rule}

The Murnaghan-Nakayama rule $[118,127,129]$ is a combinatorial formula for the characters $\chi_{\lambda}(\mu)$ of the symmetric group in terms of ribbon tableaux. Under the Frobenius characteristic map, there exists an analogous statement on the level of symmetric functions, which follows directly from the formula

$$
p_{r} s_{\lambda}=\sum_{\mu}(-1)^{\operatorname{height}(\mu / \lambda)} s_{\mu}
$$

Here $p_{r}$ is the $r$-th power sum symmetric function, $s_{\lambda}$ is the Schur function labeled by partition $\lambda$, and the sum is over all partitions $\lambda \subseteq \mu$ for which $\mu / \lambda$ is a border strip of size $r$. Recall that a border strip is a connected skew shape without any $2 \times 2$ squares. The height height $(\mu / \lambda)$ of a border strip $\mu / \lambda$ is one less than the number of rows.

In [7], an analogue of the Murnaghan-Nakayama rule for the product of $p_{r}$ times $s_{\lambda}^{(k)}$ is given. This is derived using the $k$-Pieri rule, and is expressed in terms of the action of the affine symmetric group (resp. nil-Coxeter group) on cores. To give the precise result we need to make a couple of definitions. We define a vertical domino in a skew-partition to be a pair of cells in the diagram, with one sitting directly above the other. For the skew of two $k$-bounded partitions $\lambda \subseteq \mu$ we define the height as

$$
\text { height }(\mu / \lambda)=\text { number of vertical dominos in } \mu / \lambda \text {. }
$$

For ribbons, that is skew shapes without any $2 \times 2$ squares, the definition of height can be restated as the number of occupied rows minus the number of connected components. Notice that this is compatible with the usual definition of the height of a border strip.

Definition 5.1. The skew of two $k$-bounded partitions, $\mu / \lambda$, is called a $k$-ribbon of size $r$ if $\mu$ and $\lambda$ satisfy the following properties:

(0) (containment condition) $\lambda \subseteq \mu$ and $\lambda^{\omega_{k}} \subseteq \mu^{\omega_{k}}$;

(1) (size condition) $|\mu / \lambda|=r$;

(2) (ribbon condition) $\mathfrak{c}(\mu) / \mathfrak{c}(\lambda)$ is a ribbon; 
(3) (connectedness condition) $\mathfrak{c}(\mu) / \mathfrak{c}(\lambda)$ is $k$-connected, that is, the contents of $\mathfrak{c}(\mu) / \mathfrak{c}(\lambda)$ form an interval of $[0, k]$ (where 0 is $k$ are adjacent);

(4) (height statistics condition) height $(\mu / \lambda)+\operatorname{height}\left(\mu^{\omega_{k}} / \lambda^{\omega_{k}}\right)=r-1$.

Then the $k$-Murnaghan-Nakayama rule states:

Theorem 5.2. For $1 \leq r \leq k$ and $\lambda$ a $k$-bounded partition, we have

$$
p_{r} s_{\lambda}^{(k)}=\sum_{\mu}(-1)^{\operatorname{height}(\mu / \lambda)} s_{\mu}^{(k)}
$$

where the sum is over all $k$-bounded partitions $\mu$ such that $\mu / \lambda$ is a k-ribbon of size $r$.

Computer evidence suggests that the ribbon condition (2) of Definition 5.1 might be superfluous because it is implied by the other conditions of the definition. This was checked for $k, r \leq 11$ and for all $|\lambda|=n \leq 12$ and $|\mu|=n+r$. Also, the $k$-Murnaghan-Nakayama rule of Theorem 5.2 was only proven for the definition of $k$-Schur functions $s_{\lambda}^{(k)}[X ; 1]$ and not in terms of $A_{\lambda}^{(k)}[X ; 1]$ or $\tilde{A}_{\lambda}^{(k)}[X ; 1]$.

Note that a Murnaghan-Nakayama rule potentially provides us with a fourth, independent definition of the $k$-Schur functions in a manner similar to Equation (2.19). The fault with this approach is that it is not immediately obvious that this system of equations is invertible and consequently defines the elements $s_{\lambda}^{(k)}$.

An analog of the Murnaghan-Nakayama rule for the elements $\tilde{F}_{\lambda}^{(k)}[X]$ would give a combinatorial interpretation of the $k$-Schur functions in the power sum basis at $t=1$.

Sage Example 5.3. Let us show how to compute the Murnaghan-Nakayama rule for $\tilde{F}_{\lambda}^{(k)}[X]$. The quotient space $\Lambda^{(k)}$ is implemented in SAGE, but there are several means of computing the coefficients of $p_{k} \tilde{F}_{\lambda}^{(k)}[X]$ by duality. As an example, let us compute $p_{2} \tilde{F}_{21}^{(3)}[X]:$

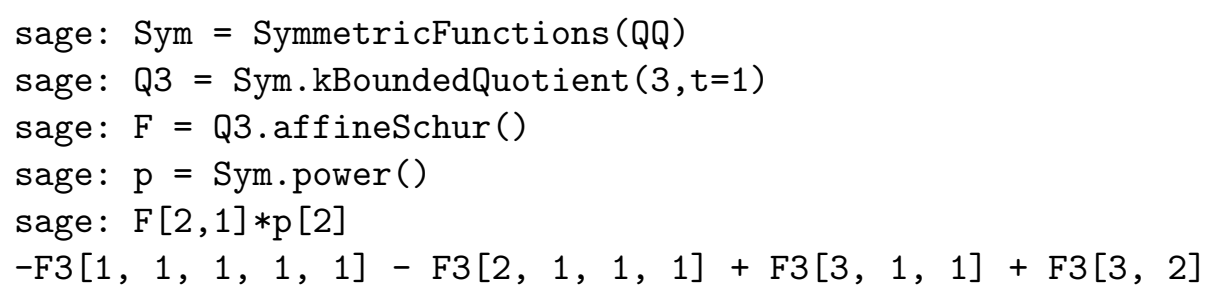

Hence this computation shows that

$$
p_{2} \tilde{F}_{21}^{(3)}=\tilde{F}_{32}^{(3)}+\tilde{F}_{311}^{(3)}-\tilde{F}_{2111}^{(3)}-\tilde{F}_{11111}^{(3)}
$$




\subsection{A rectangle generalization at $t$ a root of unity}

Let $\zeta_{m}$ be an $m^{\text {th }}$ root of unity (take $\zeta_{m}=e^{2 \pi i / m}$ ). A result due to Lascoux, Leclerc and Thibon [104] states that if $\lambda=\left(1^{m_{1}}, 2^{m_{2}}, \ldots, d^{m_{d}}\right)$ and $m_{i}=q_{i} m+r_{i}$ for $0 \leq r_{i}<m$, then

$$
Q_{\lambda}^{\prime}\left[X ; \zeta_{m}\right]=\left(Q_{\left(1^{m}\right)}^{\prime}\left[X ; \zeta_{m}\right]\right)^{q_{1}}\left(Q_{\left(2^{m}\right)}^{\prime}\left[X ; \zeta_{m}\right]\right)^{q_{2}} \cdots\left(Q_{\left(d^{m}\right)}^{\prime}\left[X ; \zeta_{m}\right]\right)^{q_{d}} Q_{\nu}^{\prime}\left[X ; \zeta_{m}\right]
$$

where $\nu=\left(1^{r_{1}}, 2^{r_{2}}, \ldots, d^{r_{d}}\right)$. A similar property is shown by Descouens and Morita [39] for the Macdonald symmetric functions. Namely they show

$$
H_{\lambda}\left[X ; q, \zeta_{m}\right]=\left(H_{\left(1^{m}\right)}\left[X ; q, \zeta_{m}\right]\right)^{q_{1}}\left(H_{\left(2^{m}\right)}\left[X ; q, \zeta_{m}\right]\right)^{q_{2}} \cdots\left(H_{\left(d^{m}\right)}\left[X ; q, \zeta_{m}\right]\right)^{q_{d}} H_{\nu}\left[X ; q, \zeta_{m}\right]
$$

Moreover it is shown in these references that

$$
Q_{\left(r^{m}\right)}^{\prime}\left[X ; \zeta_{m}\right]=p_{m} \circ h_{r}
$$

and

$$
H_{\left(r^{m}\right)}\left[X ; q, \zeta_{m}\right]=p_{m} \circ h_{r}[X /(1-q)]\left(\prod_{i=1}^{r}\left(1-q^{i m}\right)\right)
$$

where $\circ$ is the operation of plethysm.

Since at arbitrary $t$, both of these functions expand positively in $k$-Schur functions, it is natural to ask if this property is shared by the $k$-Schur functions themselves. At $t=1$ the $k$-Schur functions satisfy (see Section 4.6)

$$
s_{\left(\ell^{k-\ell+1}\right)}^{(k)}[X ; 1] s_{\lambda}^{(k)}[X ; 1]=s_{\left(\ell^{k-\ell+1}\right) \cup \lambda}^{(k)}[X ; 1]
$$

At an $m^{\text {th }}$ root of unity this property seems to generalize and we conjecture

Conjecture 5.4. For $\ell \leq k$ and $\zeta_{m}=e^{2 \pi i / m}$

$$
s_{\left(\ell^{m(k-\ell+1)}\right)}^{(k)}\left[X ; \zeta_{m}\right] s_{\lambda}^{(k)}\left[X ; \zeta_{m}\right]=s_{\left(\ell^{m(k-\ell+1)}\right) \cup \lambda}^{(k)}\left[X ; \zeta_{m}\right]
$$

and moreover

$$
s_{\left(\ell^{m(k-\ell+1)}\right)}^{(k)}\left[X ; \zeta_{m}\right]=p_{m} \circ s_{\left(\ell^{k-\ell+1}\right)}^{(k)}[X ; 1] .
$$

Sage Example 5.5. We demonstrate an example of this conjecture by building two copies of the $k$-Schur functions in SAGE, one where the parameter $t$ is specialized to a fourth root of unity, and the other where $t=1$. Expanding these $k$-Schur functions in the power sum basis makes it possible to see the relationship between these elements, checking the second relation: 


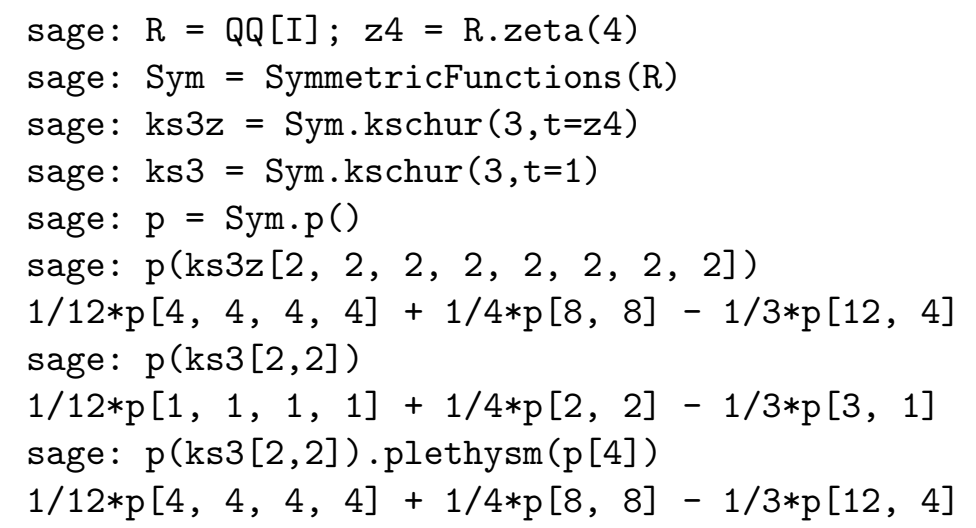

The first relation can be checked as follows:

sage: $\operatorname{ks} 3 z[3,3,3,3] * k s 3 z[2,1]$

$\mathrm{ks} 3[3,3,3,3,2,1]$

\subsection{A dual-basis to $s_{\lambda}^{(k)}[X ; t]$}

Recall from Section 1.7 that $P_{\lambda}[X ; t]$ is the dual basis to $Q_{\lambda}^{\prime}[X ; t]$ with respect to the $\langle.,$. scalar product. Moreover, we have the expansion (3.13)

$$
Q_{\mu}^{\prime}[X ; t]=\sum_{\lambda \vdash|\mu|, \lambda_{1} \leq k} K_{\lambda \mu}^{(k)}(t) \tilde{s}_{\lambda}^{(k)}[X ; t]
$$

and

$$
\tilde{s}_{\lambda}^{(k)}[X ; t]=\sum_{\mu \vdash|\lambda|, \mu_{1} \leq k} K_{\mu \lambda}^{(k)}(t)^{-1} Q_{\mu}^{\prime}[X ; t]
$$

where $K_{\lambda \mu}^{(k)}(t) t$-enumerate weak tableaux of shape $\mathfrak{c}(\lambda)$ and weight $\mu$. A $t$-generalization for dual $k$-Schur functions of Equation (2.17) then comes out of this [36] by duality,

$$
\tilde{F}_{\lambda}^{(k)}[X ; t]:=\sum_{\mu \vdash|\lambda|, \mu_{1} \leq k} K_{\lambda \mu}^{(k)}(t) P_{\mu}[X ; t]
$$

These elements clearly live in a space spanned by $\left\{P_{\lambda}[X ; t]\right\}_{\lambda_{1} \leq k}$. In fact, by triangularity considerations of the symmetric functions $P_{\lambda}[X ; t]$, we have that

$$
\Lambda_{t}^{(k)}:=\mathcal{L}\left\{\tilde{F}_{\lambda}^{(k)}[X ; t]\right\}_{\lambda_{1} \leq k}=\mathcal{L}\left\{P_{\lambda}[X ; t]\right\}_{\lambda_{1} \leq k}=\mathcal{L}\left\{s_{\lambda}[X]\right\}_{\lambda_{1} \leq k}=\mathcal{L}\left\{m_{\lambda}[X]\right\}_{\lambda_{1} \leq k} .
$$

While this space is not closed under the usual product, it is closed under coproduct. 
Now recall from Section 1.7 , that $\left\langle Q_{\lambda}^{\prime}[X ; t], P_{\mu}[X ; t]\right\rangle=\delta_{\lambda \mu}$. Hence

$$
\begin{aligned}
\left\langle s_{\mu}^{(k)}[X ; t], \tilde{F}_{\lambda}^{(k)}[X ; t]\right\rangle & =\sum_{\substack{\gamma \vdash|\mu| \\
\gamma \\
\gamma_{1} \leq k}} K_{\gamma \mu}^{(k)}(t)^{-1}\left\langle Q_{\gamma}^{\prime}[X ; t], \tilde{F}_{\lambda}^{(k)}[X ; t]\right\rangle \\
& =\sum_{\substack{\gamma \vdash|\mu| \\
\gamma_{1} \leq k}} K_{\gamma \mu}^{(k)}(t)^{-1} K_{\lambda \gamma}^{(k)}(t)=\delta_{\lambda \mu} .
\end{aligned}
$$

Therefore we can see that the elements $\left\{\tilde{F}_{\lambda}^{(k)}[X ; t]\right\}_{\lambda_{1} \leq k}$ are another $t$-analogue of the Schur functions which, by triangularity considerations, live in the linear span of $\mathcal{L}\left\{m_{\lambda}: \lambda_{1} \leq k\right\}$ and are dual to $\left\{s_{\lambda}^{(k)}[X ; t]\right\}_{\lambda_{1} \leq k}$ with respect to the usual scalar product.

Just as with the space $\Lambda_{(k)}^{t}$, the linear span of the dual elements for the $k$-Schur functions is a subspace and not an algebra with respect to the usual product. We can however make it an algebra, by introducing a product

$$
\tilde{F}_{\nu}^{(k)}[X ; t] \cdot{ }^{t} \tilde{F}_{\mu}^{(k)}[X ; t]:=\sum_{\lambda} C_{\nu \mu}^{\lambda(k)}(t) \tilde{F}_{\lambda}^{(k)}[X ; t]
$$

where the coefficients $C_{\nu \mu}^{\lambda(k)}(t)$ are precisely those defined by Equation (4.9). The coefficients $C_{\mu \nu}^{\lambda(k)}(t)$ are discussed in Section 4.8 and they are conjectured to be polynomials in $t$ with non-negative integer coefficients. To be clear, we take as definition that the space is closed under a product where the structure coefficients are

$$
C_{\nu \mu}^{\lambda(k)}(t)=\left\langle\Delta\left(s_{\lambda}^{(k)}[X ; t]\right), \tilde{F}_{\nu}^{(k)}[X ; t] \tilde{F}_{\mu}^{(k)}[Y ; t]\right\rangle
$$

There are several ways of computing a more explicit formula for these coefficients, but let us consider one that can be found by expanding the elements $s_{\lambda}^{(k)}[X ; t]$ and $\tilde{F}_{\lambda}^{(k)}[X ; t]$ in the Schur basis. Since

$$
s_{\lambda}^{(k)}[X ; t]=\sum_{\mu \vdash|\lambda|}\left\langle s_{\lambda}^{(k)}[X ; t], s_{\mu}[X]\right\rangle s_{\mu}[X]
$$

and

$$
\tilde{F}_{\lambda}^{(k)}[X ; t]=\sum_{\mu \vdash|\lambda|}\left\langle\tilde{F}_{\lambda}^{(k)}[X ; t], s_{\mu}[X]\right\rangle s_{\mu}[X]
$$

a formula for these coefficients is found by combining (5.8), (5.9), and (5.10) to obtain

$$
C_{\nu \mu}^{\lambda(k)}(t)=\sum_{\substack{\theta \vdash|\lambda| \\ \tau \vdash|\nu|, \gamma \vdash|\mu|}} c_{\tau \gamma}^{\theta}\left\langle s_{\lambda}^{(k)}[X ; t], s_{\theta}[X]\right\rangle\left\langle\tilde{F}_{\nu}^{(k)}[X ; t], s_{\tau}[X]\right\rangle\left\langle\tilde{F}_{\mu}^{(k)}[X ; t], s_{\gamma}[X]\right\rangle
$$


where the $c_{\mu \nu}^{\lambda}$ are the Littlewood-Richardson coefficients previously discussed.

Little is known about this product and it would be useful to understand it in terms of another basis. However, it is possible to compute these coefficients as elements of a quotient algebra or, as we do here, define a projection operator and notice that the product ${ }^{t}{ }^{t}$ is simply the usual product followed by a projection into the space $\Lambda_{t}^{(k)}$.

Proposition 5.6. Let $\Theta^{(k)}$ be a projection from $\Lambda$ to $\Lambda_{t}^{(k)}$, the space spanned by functions dual to the $k$-Schur functions, defined by $\Theta^{(k)}\left(P_{\lambda}[X ; t]\right)=P_{\lambda}[X ; t]$ if $\lambda_{1} \leq k$, and $\Theta^{(k)}\left(P_{\lambda}[X ; t]\right)=0$ if $\lambda_{1}>k$, then

$$
\tilde{F}_{\nu}^{(k)}[X ; t] \cdot{ }^{t} \tilde{F}_{\mu}^{(k)}[X ; t]=\Theta^{(k)}\left(\tilde{F}_{\nu}^{(k)}[X ; t] \tilde{F}_{\mu}^{(k)}[X ; t]\right) .
$$

Proof. In order to prove this we need to show that the coefficient of $\tilde{F}_{\lambda}^{(k)}[X ; t]$ in the expression $\Theta^{(k)}\left(\tilde{F}_{\nu}^{(k)}[X ; t] \tilde{F}_{\mu}^{(k)}[X ; t]\right)$ is equal to $C_{\nu \mu}^{\lambda(k)}(t)$. Since $s_{\lambda}^{(k)}[X ; t]$ is in the linear span of elements $\left\{Q_{\lambda}^{\prime}[X ; t]\right\}_{\lambda_{1} \leq k}$, we can conclude that the coefficient of $\tilde{F}_{\lambda}^{(k)}[X ; t]$ in $\Theta^{(k)}\left(\tilde{F}_{\nu}^{(k)}[X ; t] \tilde{F}_{\mu}^{(k)}[X ; t]\right)$ is equal to

$$
\left\langle\Theta^{(k)}\left(\tilde{F}_{\nu}^{(k)}[X ; t] \tilde{F}_{\mu}^{(k)}[X ; t]\right), s_{\lambda}^{(k)}[X ; t]\right\rangle=\left\langle\tilde{F}_{\nu}^{(k)}[X ; t] \tilde{F}_{\mu}^{(k)}[X ; t], s_{\lambda}^{(k)}[X ; t]\right\rangle .
$$

We can use Equations (5.9) and (5.10) to expand the right hand side so that it is equal to

$$
\begin{aligned}
& \sum_{\substack{\theta \vdash|\lambda| \\
\tau \vdash|\nu|, \gamma \vdash|\mu|}}\left\langle s_{\tau} s_{\gamma}, s_{\theta}\right\rangle\left\langle\tilde{F}_{\nu}^{(k)}[X ; t], s_{\tau}[X]\right\rangle\left\langle\tilde{F}_{\mu}^{(k)}[X ; t], s_{\gamma}[X]\right\rangle\left\langle s_{\lambda}^{(k)}[X ; t], s_{\theta}[X]\right\rangle \\
= & \sum_{\substack{\theta \vdash|\lambda| \\
\tau \vdash|,|, \gamma \vdash|\mu|}} c_{\tau \gamma}^{\theta}\left\langle\tilde{F}_{\nu}^{(k)}[X ; t], s_{\tau}[X]\right\rangle\left\langle\tilde{F}_{\mu}^{(k)}[X ; t], s_{\gamma}[X]\right\rangle\left\langle s_{\lambda}^{(k)}[X ; t], s_{\theta}[X]\right\rangle=C_{\nu \mu}^{\lambda(k)}(t)
\end{aligned}
$$

by (5.11). This shows that the coefficients that appear in the $t$-product in Equation (5.7) are the same that appear by usual multiplication followed by a projection by $\Theta^{(k)}$.

Sage Example 5.7. We can compute the coefficients $C_{\lambda \mu}^{\nu(k)}(t)$ as structure coefficients of the dual basis elements $\tilde{F}_{\lambda}^{(k)}[X ; t]$. These elements are implemented in SAGE in a space representing the quotient of the ring of symmetric functions $\Lambda$ by the ideal generated by the Hall-Littlewood symmetric functions $P_{\lambda}[X ; t]$ with $\lambda_{1}>k$.

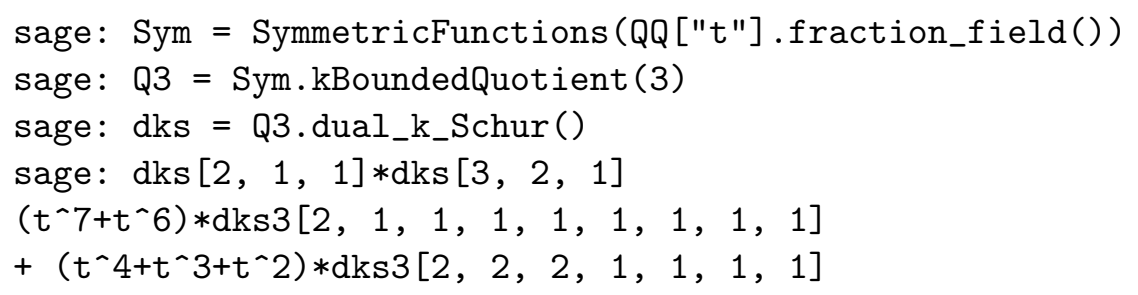


$+\left(t^{\wedge} 3+t^{\wedge} 2\right) * \operatorname{dks} 3[2,2,2,2,1,1]$

$+(t \wedge 5+2 * t \wedge 4+2 * t \wedge 3+t \wedge 2) * \operatorname{dks} 3[2,2,2,2,2]$

$+(t \wedge 5+2 * t \wedge 4+t \wedge 3) * \operatorname{dks} 3[3,1,1,1,1,1,1,1]$

$+\left(2 * t \wedge 5+3 * t \wedge 4+4 * t \wedge 3+3 * t^{\wedge} 2+t\right) * d k s 3[3,2,1,1,1,1,1]$

$+(2 * t \wedge 2+t+1) * d k s 3[3,2,2,1,1,1]$

$+(t \wedge 4+3 * t \wedge 3+4 * t \wedge 2+3 * t+1) * \operatorname{dks} 3[3,2,2,2,1]$

$+(t \wedge 5+t \wedge 4+4 * t \wedge 3+4 * t \wedge 2+3 * t+1) * d k s 3[3,3,1,1,1,1]$

$+(2 * t \wedge 5+3 * t \wedge 4+5 * t \wedge 3+6 * t \wedge 2+4 * t+2) * \operatorname{dks} 3[3,3,2,1,1]$

$+(t \wedge 4+t \wedge 3+3 * t \wedge 2+2 * t+1) * \operatorname{dks} 3[3,3,2,2]$

$+\left(t^{\wedge} 5+3 * t \wedge 4+3 * t \wedge 3+4 * t \wedge 2+2 * t+1\right) * \operatorname{dks} 3[3,3,3,1]$

\subsection{A product on $\Lambda_{(k)}^{t}$}

What is interesting about the $\tilde{F}_{\lambda}^{(k)}[X ; t]$ elements is that they are clearly closed under the usual coproduct operation of the symmetric functions. It is therefore natural to consider the coefficients that appear in the coproduct of the elements which are dual to the $k$-Schur functions as the structure constants of the product of $k$-Schur functions.

Define the coefficients $c_{\nu \mu}^{\lambda(k)}(t)$ by the coproduct formula

$$
\Delta\left(\tilde{F}_{\lambda}^{(k)}[X ; t]\right)=\sum_{\nu, \mu} c_{\nu \mu}^{\lambda(k)}(t) \tilde{F}_{\nu}^{(k)}[X ; t] \tilde{F}_{\mu}^{(k)}[Y ; t]
$$

We will give a more precise calculation of these coefficients below, but assuming that they exist, we then define

$$
s_{\nu}^{(k)}[X ; t] \cdot t s_{\mu}^{(k)}[X ; t]:=\sum_{\lambda \vdash|\nu|+|\mu|} c_{\nu \mu}^{\lambda(k)}(t) s_{\lambda}^{(k)}[X ; t]
$$

where

$$
c_{\nu \mu}^{\lambda(k)}(t):=\left\langle\Delta\left(\tilde{F}_{\lambda}^{(k)}[X ; t]\right), s_{\nu}^{(k)}[X ; t] s_{\mu}^{(k)}[Y ; t]\right\rangle .
$$

We can then derive from Equations (5.9) and (5.10), that

$$
c_{\nu \mu}^{\lambda(k)}(t)=\sum_{\substack{\theta \vdash|\lambda| \\ \tau \vdash|\nu|, \gamma \vdash|\mu|}} c_{\tau \gamma}^{\theta}\left\langle\tilde{F}_{\lambda}^{(k)}[X ; t], s_{\theta}[X]\right\rangle\left\langle s_{\nu}^{(k)}[X ; t], s_{\tau}[X]\right\rangle\left\langle s_{\mu}^{(k)}[X ; t], s_{\gamma}[X]\right\rangle .
$$

At this point it is possible to see that while it is not obvious what the product structure on the $k$-Schur functions should be, if we take this to be the definition then, as in the case with the product $\cdot^{t}$, the product on $k$-Schur functions can also be realized as a projection of the usual product. 
Proposition 5.8. Let $\Theta_{(k)}$ be a projection from the space $\Lambda$ to the space $\Lambda_{(k)}^{t}$ spanned by the $k$-Schur functions defined by $\Theta_{(k)}\left(Q_{\lambda}^{\prime}[X ; t]\right)=Q_{\lambda}^{\prime}[X ; t]$ if $\lambda_{1} \leq k$ and $\Theta_{(k)}\left(Q_{\lambda}^{\prime}[X ; t]\right)=0$, then

$$
s_{\nu}^{(k)}[X ; t] \cdot{ }_{t} s_{\mu}^{(k)}[X ; t]=\Theta_{(k)}\left(s_{\nu}^{(k)}[X ; t] s_{\mu}^{(k)}[X ; t]\right) .
$$

Proof. The proof proceeds exactly as it did in Proposition 5.6. We compute that the coefficient of $s_{\lambda}^{(k)}[X ; t]$ in $\Theta_{(k)}\left(s_{\nu}^{(k)}[X ; t] s_{\mu}^{(k)}[X ; t]\right)$ is

$$
\begin{aligned}
& \left\langle\Theta_{(k)}\left(s_{\nu}^{(k)}[X ; t] s_{\mu}^{(k)}[X ; t]\right), \tilde{F}_{\lambda}^{(k)}[X ; t]\right\rangle=\left\langle s_{\nu}^{(k)}[X ; t] s_{\mu}^{(k)}[X ; t], \tilde{F}_{\lambda}^{(k)}[X ; t]\right\rangle \\
= & \sum_{\substack{\theta \vdash|\lambda| \\
\tau \vdash|\nu|, \gamma \vdash|\mu|}} c_{\tau \gamma}^{\theta}\left\langle s_{\nu}^{(k)}[X ; t], s_{\tau}[X]\right\rangle\left\langle s_{\mu}^{(k)}[X ; t], s_{\gamma}[X]\right\rangle\left\langle\tilde{F}_{\lambda}^{(k)}[X ; t], s_{\theta}[X]\right\rangle=c_{\nu \mu}^{\lambda(k)}(t) .
\end{aligned}
$$

So we see again that the structure coefficients in the definition of the $t$-product in Equation (5.14) are exactly those that occur by taking the usual product and then projecting using the map $\Theta_{(k)}$.

It was discussed in Section 4.7 that the coefficients $c_{\mu \nu}^{\lambda(k)}(1)$ are non-negative integers (conjecturally for certain definitions), but in the following example we will see that $c_{\mu \nu}^{\lambda(k)}(t)$ are not generally elements of $\mathbb{N}[t]$. This makes us believe that this product is not 'the' product to define on the space $\Lambda_{(k)}^{t}$ (if there is such a product). At least in certain cases, the operators $\mathbf{B}_{\lambda}$ from Equation (3.7) also provide a means of defining a $t$-analogue of multiplication and in light of Equation (4.1), it seems possible that there is some other $t$-product on $\Lambda_{(k)}^{t}$ which is the right one to consider on this space.

Sage Example 5.9. We show how a product of $k$-Schur functions can be computed under the projection $\Theta_{(k)}$. The product is first computed in the $Q_{\mu}^{\prime}[X ; t]$ basis and then the projection is computed by restricting the support to those partitions whose parts are less than or equal to $k$, which is 2 in this example.

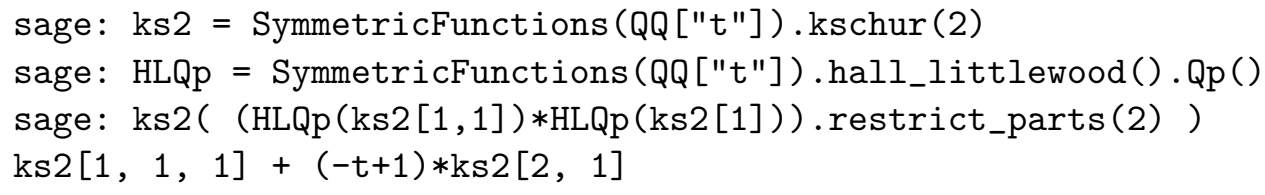

The coefficient $t-1$ should appear as the coefficient of $\tilde{F}_{1}^{(2)} \otimes \tilde{F}_{11}^{(2)}$ in the expansion of the coproduct $\Delta\left(\tilde{F}_{21}^{(2)}\right)$. We can calculate this using SAGE using the following commands.

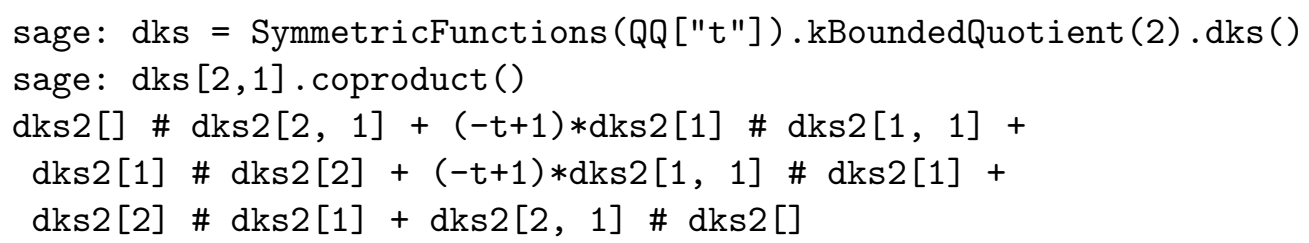




\subsection{A representation theoretic model of $k$-Schur functions}

The Frobenius map is a map from $S_{m}$-modules to symmetric functions of degree $m$ which takes an irreducible module indexed by the partition $\lambda$ to the Schur function also indexed by the partition $\lambda \vdash m$. Here we denote this map by $\mathcal{F}$ with $\mathcal{F}\left(V_{\lambda}\right)=s_{\lambda}$, where $V_{\lambda}$ is an irreducible $S_{m}$-module.

The parameter $t$ in the ring of symmetric functions represents a grading and we can define for a graded module $V=\bigoplus_{d \geq 0} V_{d}$,

$$
\mathcal{F}_{t}(V)=\sum_{d \geq 0} t^{d} \mathcal{F}\left(V_{d}\right)
$$

For example, if we consider the ring of polynomials $\mathbb{C}\left[a_{1}, a_{2}, \ldots, a_{m}\right]$ as a module graded by the degree in the variables $a_{i}$, then $\mathcal{F}_{t}\left(\mathbb{C}\left[a_{1}, a_{2}, \ldots, a_{m}\right]\right)=h_{m}\left[\frac{X}{1-t}\right]$. In particular, we also have for any irreducible $S_{m}$-module $V_{\lambda}$ that the tensor product $V_{\lambda} \otimes \mathbb{C}\left[a_{1}, a_{2}, \ldots, a_{m}\right]$ is an $S_{m}$-module, where $S_{m}$ acts diagonally on the tensors and

$$
\mathcal{F}_{t}\left(V_{\lambda} \otimes \mathbb{C}\left[a_{1}, a_{2}, \ldots, a_{m}\right]\right)=s_{\lambda}\left[\frac{X}{1-t}\right] .
$$

Mark Haiman and Li-Chung Chen [32] defined $\mathcal{M}^{(k)}$ to be the category of graded finitely generated $\mathbb{C}\left[a_{1}, a_{2}, \ldots, a_{m}\right] * S_{m}$-modules $V$ such that $V$ has an $S_{m}$-equivariant $\mathbb{C}\left[a_{1}, a_{2}, \ldots, a_{m}\right]$ free resolution using only the $V_{\lambda} \otimes \mathbb{C}\left[a_{1}, a_{2}, \ldots, a_{m}\right]$ with $\lambda_{1} \leq k$. Then they conjecture the following.

Conjecture 5.10. The modules which are irreducible in $\mathcal{M}^{(k)}$ have images under the map $\mathcal{F}_{t}$ which are equal to $\omega s_{\lambda}^{(k)}[X ; t]$ for $\lambda \vdash m$ and $\lambda_{1} \leq k$.

We say that two partitions $\lambda$ and $\mu$ of the same size are called skew-linked if there exists a skew partition $\gamma / \tau$ such that $\lambda_{i}$ is the number of cells in the $i^{\text {th }}$ row of $\gamma / \tau$ and $\mu_{i}^{\prime}$ is the number of cells in the $i^{\text {th }}$ column of $\gamma / \tau$. The definition of skew-linked is not associated with a particular value of $k$, but we have seen examples of partitions which are skew-linked through the $(k+1)$-cores. We always have that $\lambda$ and $\left(\lambda^{\omega_{k}}\right)^{\prime}$ are skew-linked since the skew partition representing the cells of $\mathfrak{c}_{k}(\lambda)$ with hook less than $k+1$ has $\lambda_{i}$ cells in row $i$ and the transpose of $\mathfrak{c}_{k}(\lambda)$ has $\lambda_{i}^{\omega_{k}}$ cells with hook less than $k+1$ in row $i$. But there are other examples of pairs of partitions which are skew-linked. Of course, if $\lambda$ and $\mu$ are skew-linked, then $\mu^{\prime}$ and $\lambda^{\prime}$ are skew-linked.

Example 5.11. For a small example, consider that the partition $(2,2,1)$ is skew-linked to $(2,2,1),(3,2),(4,1)$ and $(5)$ through the skew partitions $(2,2,1),(3,2,1) /(1),(4,2,1) /(2)$, and $(5,3,1) /(3,1)$ respectively.

For a larger example, the partition $(4,4,2,2,2,1)$ is skew linked to $(6,4,2,2,1)$ because the skew partition $(6,4,2,2,2,1) /(2)$ has rows given by $(4,4,2,2,2,1)$ and columns given by the partition $(5,4,2,2,1,1)$. 


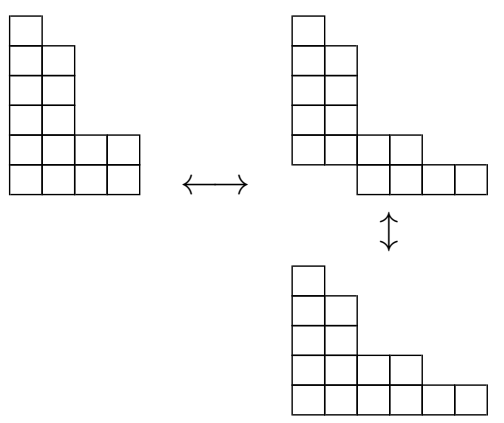

Notice that $(6,4,2,2,1)$ is not the conjugate of the $k$-conjugate of $(4,4,2,2,2,1)$ for any $k$.

Theorem 5.12. (L.-C. Chen) If $\lambda$ is skew-linked to $\mu$ by a skew partition $\gamma / \tau$ with $\tau \vdash d$, then $V_{\lambda}$ occurs with multiplicity 1 at degree $d$ in $V_{\mu} \otimes \mathbb{C}\left[a_{1}, a_{2}, \ldots, a_{m}\right]$ and does not appear at a lower degree.

As a corollary they construct a module by moding out the module $V_{\mu} \otimes \mathbb{C}\left[a_{1}, a_{2}, \ldots, a_{m}\right]$ by the space generated by all modules $V_{\gamma}$ which are at degree $d$ which are not $V_{\lambda}$. In the particular case when $\lambda$ is a $k$-bounded partition, $\lambda^{\prime}$ is skew-linked to $\mu=\lambda^{\omega_{k}}$ and the module $V_{\lambda}$ is conjectured to have Frobenius image equal to $\omega s_{\lambda}^{(k)}[X ; t]$.

Conjecture 5.13. For a $k$-bounded partition $\lambda$, let $d$ be the number of cells in $\mathfrak{c}_{k}(\lambda)$ which have a hook length greater than $k$. Let $W$ be the module which is generated by $V_{\lambda^{\omega_{k}}}$ as an $S_{m} * \mathbb{C}\left[a_{1}, a_{2}, \ldots, a_{m}\right]$-module and cogenerated by the copy of $V_{\lambda^{\prime}}$ of degree $d$. Then $\mathcal{F}_{t}(W)=\omega s_{\lambda}^{(k)}[X ; t]$.

Haiman and Li-Chung Chen [32] note that this module is only conjectured to lie within the category of $\mathcal{M}^{(k)}$.

\subsection{From Pieri to $K$-theoretic $k$-Schur functions}

As mentioned in the introduction, a trend in Schubert calculus is to generalize the classical setup. The replacement of cohomology by $K$-theory is a particularly fruitful variation. Lascoux and Schützenberger introduced the Grothendieck polynomials in [108] as representatives for the $K$-theory classes determined by structure sheaves of Schubert varieties. Grothendieck polynomials have since been connected to representation theory and algebraic geometry and combinatorics is again at the forefront (e.g. [37, 76, 101, 45]). For example, the stable Grothendieck polynomials $G_{\lambda}$ are inhomogeneous symmetric polynomials whose lowest homogeneous degree component is a Schur function. Buch proved in [27] that they are the weight generating functions

$$
G_{\lambda}=\sum_{\substack{T \text { set-valued } \\ \text { shape }(T)=\lambda}}(-1)^{|\lambda|-|\operatorname{weight}(T)|} x^{\text {weight(T)}},
$$


of tableaux called set-valued tableaux. Such a tableau $T$ is a filling of each cell in a shape with a set of integers, where a set $X$ below (west of) $Y$ satisfies $\max X<(\leq) \min Y$. The weight of $T$ is $\alpha$ where $\alpha_{i}$ is the number of cells in $T$ containing an $i$. Pieri rules are given in [113] in terms of binomial numbers and a generalization for Yamanouchi tableaux gives [27] a combinatorial rule for the structure constants.

Ideas in $k$-Schur theory extend to the inhomogeneous setting providing combinatorial tools that apply to torus-equivariant $K$-theory of the affine Grassmannian of $S L_{k+1}$. Similar to the development described in Section 2.2, a close study of a Pieri rule and its iteration is carried out in [124], leading to a family of affine set-valued tableaux that are in bijection with elements of the affine nil-Hecke algebra. These simultaneously generalize set-valued tableaux and weak $k$-tableaux.

These tableaux are defined along the lines described in Remark 2.3; the semi-standard case is given by putting conditions on the reading words of the standard case. Recall from Section 3.3 that $T_{\leq x}$ is the subtableau obtained by deleting all letters larger than $x$ from $T$ and note that this is well-defined for a set-valued tableau T. A standard affine set-valued tableau $T$ of degree $n$ is then defined as a set-valued filling such that, for each $1 \leq x \leq n$, shape $\left(T_{\leq x}\right)$ is a core and the cells containing an $x$ form the set of all removable corners of $T_{\leq x}$ with the same residue.

Example 5.14. With $k=2$, the standard affine set-valued tableaux of degree 5 with shape $\mathfrak{c}(2,1,1)=(3,1,1)$ are

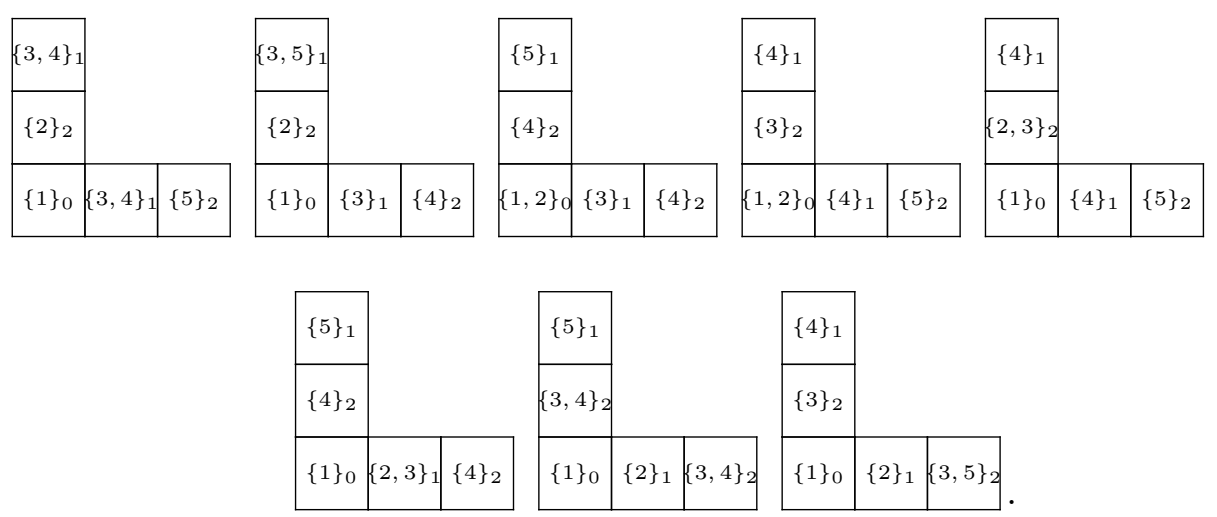

For the semi-standard case, first note that a set-valued tableau $T$ of weight $\alpha$ is a standard set-valued tableau with increasing reading words in the alphabets $\mathcal{A}_{\alpha, x}$ of (2.5), where the reading word is obtained by reading letters from a cell in decreasing order (and as usual, cells are taken from top to bottom and left to right). Since letters in standard affine set-valued tableaux can occur with multiplicity, the lowest reading word in $\mathcal{A}$ - reading the lowest occurrence of the letters in $\mathcal{A}$ from top to bottom and left to right - is used. Again, letters in the same cell are read in decreasing order. In Example 5.14, the lowest reading words in $\{1, \ldots, 5\}$ are $21435,52134,52134,32145,32145,51324,51243,41253$. 
The affine $K$-theoretic generalization of a tableau with weight $\alpha$ is then given, for any $k$-bounded composition $\alpha$, by a standard affine set-valued tableau of degree $|\alpha|$ where, for each $1 \leq x \leq \ell(\alpha)$

1. the lowest reading word in $\mathcal{A}_{\alpha, x}$ is increasing

2. the letters of $\mathcal{A}_{\alpha, x}$ occupy $\alpha_{x}$ distinct residues

3. the letters of $\mathcal{A}_{\alpha, x}$ form a horizontal strip.

Example 5.15. The affine set-valued tableaux in (5.18) of Example 5.14 all have weight $(2,1,1,1)$ and shape $(3,1,1)=\mathfrak{c}(2,1,1)$, for $k=2$.

It is proven in [124] that the weight generating functions of affine set-valued tableaux

$$
G_{\lambda}^{(k)}=\sum_{\substack{T \text { affine set-valued tableau } \\ \text { shape }(T)=\mathfrak{c}(\lambda)}}(-1)^{|\lambda|+\mid \text { weight }(T) \mid} x^{\text {weight }(T)}
$$

are Schubert representatives for $K$-theory of the affine Grassmannian of $S L_{k+1}$ called affine stable Grothendieck polynomials $[78,85]$. These reduce to Grothendieck polynomials for large $k$ and the term of lowest degree in $G_{\lambda}^{(k)}$ is the dual $k$-Schur function $\tilde{F}_{\lambda}^{(k)}$. As in the $k$-Schur set-up, these do not form a self-dual basis. Instead, the dual to $\left\{G_{\lambda}^{(k)}\right\}_{\lambda_{1} \leq k}$ is an inhomogeneous basis $\left\{g_{\lambda}^{(k)}\right\}_{\lambda_{1} \leq k}$ for $\Lambda_{(k)}$.

The set-up discussed in Sections 2.1 and 2.2 is extended in [124] and gives affine $K$ theoretic properties for this basis such as Pieri rules and an analog to property (4.2) for an inhomogeneous involution $\Omega$;

$$
\Omega g_{\lambda}^{(k)}=g_{\lambda^{\omega} \omega_{k}}^{(k)}
$$

Among some of the open combinatorial problems, it remains to develop an affine $K$ theoretic set-up in the dual world along the lines discussed in Section 2.4; finding the Pieri rules for $\left\{G_{\lambda}^{(k)}\right\}_{\lambda_{1} \leq k}$ and giving a weight-generating formulation for $\left\{g_{\lambda}^{(k)}\right\}_{\lambda_{1} \leq k}$. In addition, there are properties of Grothendieck polynomials that conjecturally extend to these new bases. For example, there is a combinatorial expansion of Grothendieck polynomials into Schur functions described by a family of skew tableaux [113]. It is conjectured that $G_{\lambda}^{(k)}$ and $g_{\lambda}^{(k)}$ have combinatorial expansions in terms of dual $k$-Schur functions and $k$-Schur functions, respectively. Noncommutative versions of the affine stable Grothendieck polynomials and $\left\{g_{\lambda}^{(k)}\right\}_{\lambda_{1} \leq k}$ are given in [85], where geometric aspects of these bases are also explored. Further conjectures relating to these bases can be found in [85, 124].

Sage Example 5.16. The basis $\left\{g_{\lambda}^{(k)}\right\}_{\lambda_{1} \leq k}$ has been implemented in SAGE and so it is possible to begin experimenting with these combinatorial problems. 


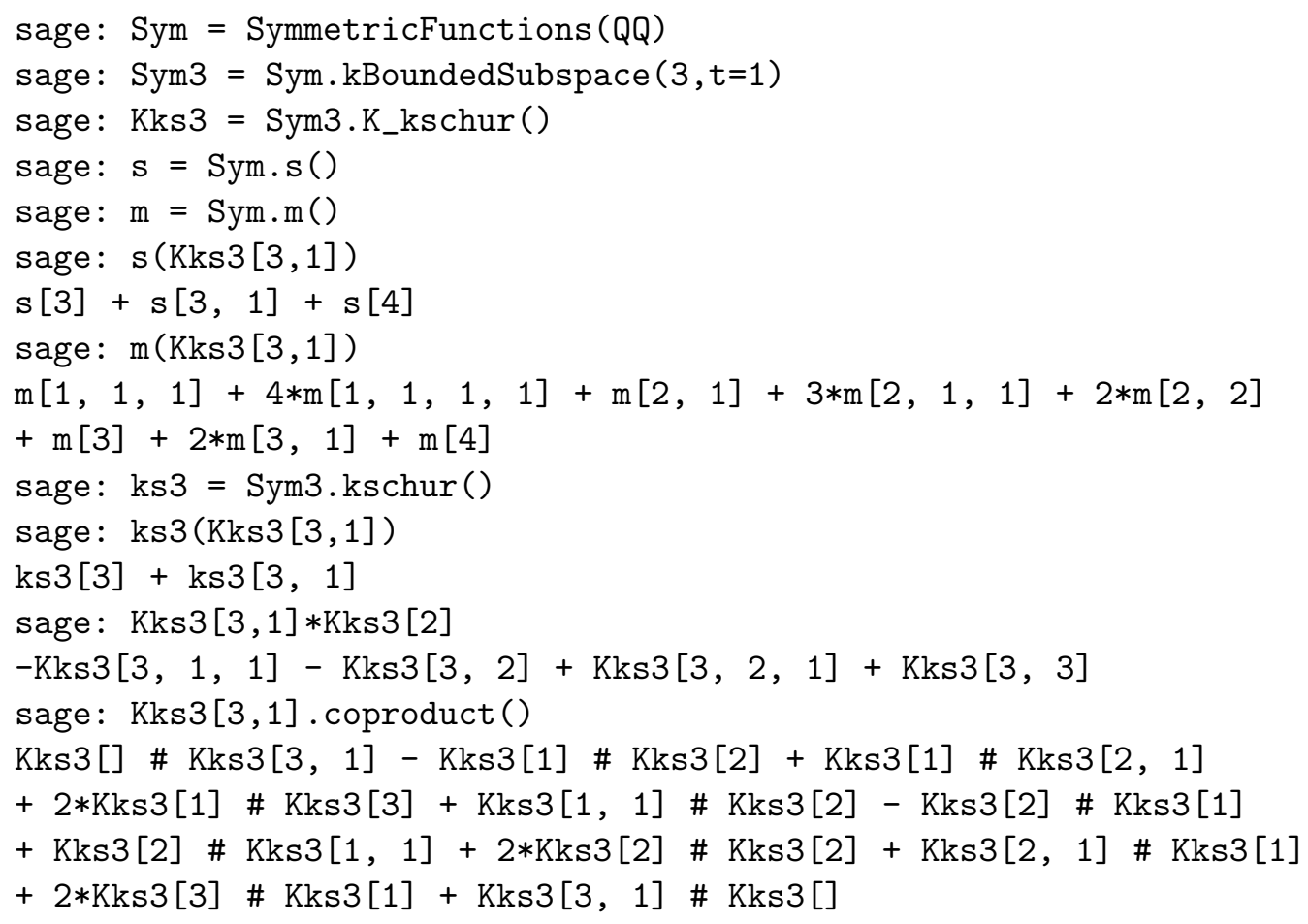

Since the elements $\left\{G_{\lambda}^{(k)}\right\}_{\lambda_{1} \leq k}$ are an infinite sum of elements of degree greater than or equal to $|\lambda|$ and we do not know their algebra structure, we cannot currently represent them as we do other bases in SAGE. However, a preliminary implementation of this basis does exist that allows one to compute the elements up to a a given degree.

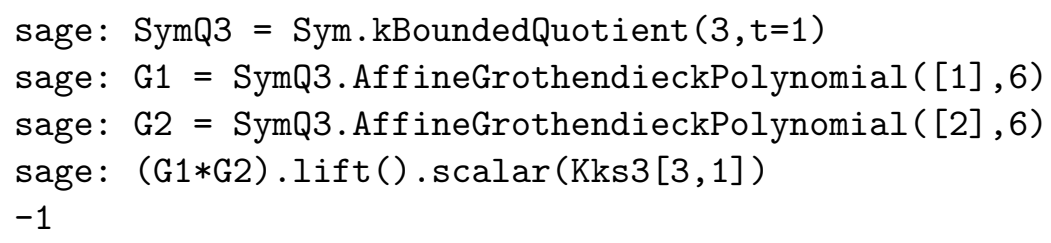

Notice how the coproduct applied to $g_{(3,1)}^{(3)}$ agrees with the product structure in this one calculation since we see that coefficient of $G_{(3,1)}^{(3)}$ in the product of $G_{(2)}^{(3)}$ and $G_{(1)}^{(3)}$ is equal to the coefficient of $g_{(2)}^{(3)} \otimes g_{(1)}^{(3)}$ in $\Delta\left(g_{(3,1)}^{(3)}\right)$.

We may also list all the terms which appear in a product of elements of $\left\{G_{\lambda}^{(k)}\right\}_{\lambda_{1} \leq k}$. This is sufficient for generating data for a Pieri rule on $\left\{G_{\lambda}^{(k)}\right\}_{\lambda_{1} \leq k}$ since we can compute products and use the duality with the $\left\{g_{\lambda}^{(k)}\right\}_{\lambda_{1} \leq k}$ basis to determine the structure coefficients up to a degree higher than what appears in our product.

sage: G31 = SymQ3.AffineGrothendieckPolynomial $([3,1], 8)$ 


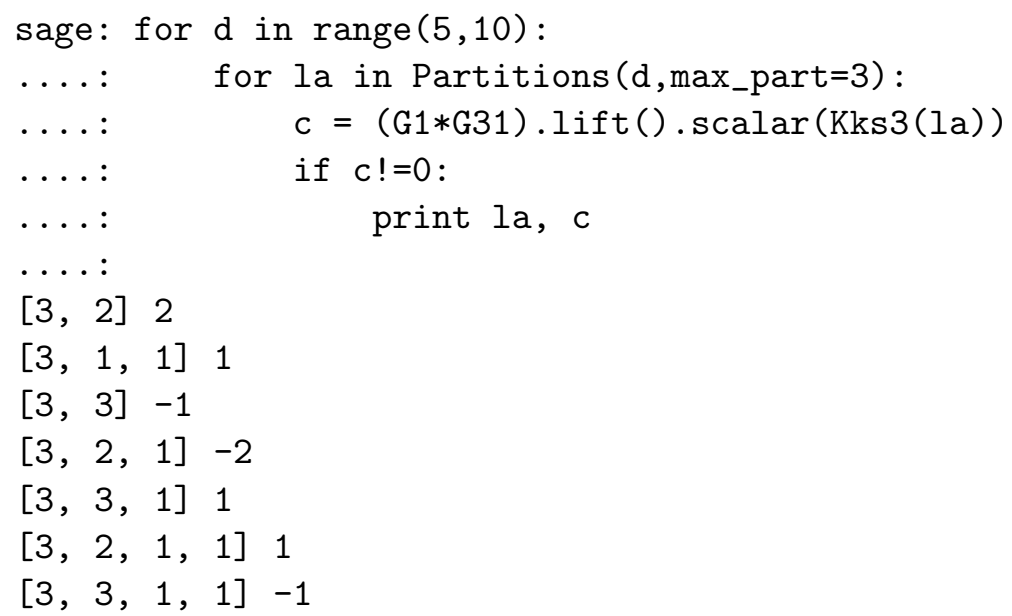

This SAgE calculation shows that no terms indexed by partitions of size 9 appear in the product of $G_{(3,1)}^{(3)}$ and $G_{(1)}^{(3)}$, and since we believe that this indicates highest degree of terms which will appear in our product will be 8 , then

$$
G_{(3,1)}^{(3)} G_{(1)}^{(3)}=2 G_{(3,2)}^{(3)}+G_{(3,1,1)}^{(3)}-G_{(3,3)}^{(3)}-2 G_{(3,2,1)}^{(3)}+G_{(3,3,1)}^{(3)}+G_{(3,2,1,1)}^{(3)}-G_{(3,3,1,1)}^{(3)} .
$$

\section{Duality between the weak and strong orders}

In this section we consider a $k$-analogue of the Cauchy identity and the Robinson-SchenstedKnuth (RSK) algorithm (or insertion algorithm). The RSK algorithm provides a bijection between permutations and pairs of tableaux satisfying certain conditions. As shown in [81] this can be generalized to the affine setting.

\section{$6.1 \quad k$-analogue of the Cauchy identity}

In the algebra of symmetric functions (or rather polynomials) an important identity is the Cauchy identity, stating that

$$
\prod_{i, j=1}^{m} \frac{1}{1-x_{i} y_{j}}=\prod_{j=1}^{m} \sum_{r \geq 0} h_{r}\left[X_{m}\right] y_{j}^{r}=\sum_{\lambda} h_{\lambda}\left[X_{m}\right] m_{\lambda}\left[Y_{m}\right]=\sum_{\lambda} s_{\lambda}\left[X_{m}\right] s_{\lambda}\left[Y_{m}\right],
$$

where the last two sums run over all partitions $\lambda$.

Although there is an algebraic proof of this identity that follows from calculations in Section 1.5, there is also a direct combinatorial proof of this result. Recall from Equation (2.7) that the Schur function is equal to

$$
s_{\lambda}\left[X_{m}\right]=\sum_{T} \mathbf{x}^{T}
$$


where the sum is over all semi-standard tableaux of shape $\lambda$ and $\mathbf{x}^{T}$ is a monomial which represents the product over $i$ of $x_{i}$ raised to the number of $i$ in the tableaux.

The Schur functions are in fact characterized as the unique basis which satisfies Equation (6.1) and which is triangularly related to the monomial symmetric functions. Notice that

$$
\prod_{i, j=1}^{m} \frac{1}{1-x_{i} y_{j}}=\sum_{M} \prod_{i, j=1}^{m}\left(x_{i} y_{j}\right)^{m_{i j}}=\sum_{M}(\mathbf{x y})^{M}
$$

where the sum is over all $m \times m$ matrices $M=\left(m_{i j}\right)_{1 \leq i, j \leq m}$ with non-negative integer entries $m_{i j}$. There is a famous bijection due to Robinson-Schensted-Knuth [72, 138, 143] (see for instance [140] for a clear exposition of the bijection) that identifies such a matrix $M$ to a biword in the alphabet of letters $\left(\begin{array}{l}r \\ s\end{array}\right)$ with $1 \leq r, s \leq m$. The bijection maps these biwords to pairs $(P, Q)$, where $P$ and $Q$ are semi-standard tableaux of the same shape and $\mathbf{x}^{P} \mathbf{y}^{Q}=\prod_{i, j=1}^{m}\left(x_{i} y_{j}\right)^{m_{i j}}$. As a consequence we conclude that

$$
\prod_{i, j=1}^{m} \frac{1}{1-x_{i} y_{j}}=\sum_{M}(\mathbf{x y})^{M}=\sum_{(P, Q)} \mathbf{x}^{P} \mathbf{y}^{Q}=\sum_{\lambda}\left(\sum_{\operatorname{shape}(P)=\lambda} \mathbf{x}^{P}\right)\left(\sum_{\operatorname{shape}(Q)=\lambda} \mathbf{y}^{Q}\right) \text {. }
$$

Since $\sum_{\text {shape }(P)=\lambda} \mathbf{x}^{P}$ is triangularly related to the monomial basis, Equation (6.2) must hold by comparing Equations (6.1) and (6.4). In this section we consider the generalization of the Cauchy identity and the RSK algorithm to $k$-Schur functions and their dual basis at $t=1$.

By taking the coefficient of $x_{1} x_{2} \cdots x_{m} y_{1} y_{2} \cdots y_{m}$ in Equation (6.1), we find the identity

$$
m !=\sum_{\lambda \vdash m} f_{\lambda}^{2}
$$

where $f_{\lambda}$ is the number of standard tableaux of shape $\lambda$. This may be seen as an algebraic formulation of the more standard presentation of the RSK algorithm on permutations, namely, there is a bijection between permutations $\pi$ and pairs of tableaux $(P, Q)$, where $P$ and $Q$ are standard tableaux of the same shape.

In Equation (2.17), we stated that $\tilde{F}_{\lambda}^{(k)}=\sum_{\mu} K_{\lambda \mu}^{(k)} m_{\mu}$, where $K_{\lambda \mu}^{(k)}$ is equal to the number of weak tableaux of shape $\mathfrak{c}(\lambda)$ and weight $\mu$. The collective results in $[78,97]$ show that

$$
\tilde{F}_{\lambda}^{(k)}\left[X_{m}\right]=\sum_{T} \mathbf{x}^{T}
$$

where the sum is over all weak tableaux $T$ of shape $\mathfrak{c}(\lambda)$ in the weight $\{1,2, \ldots, m\}$.

Now consider the following $k$-bounded analogue of the kernel (6.3) given by

$$
\prod_{j=1}^{m}\left(1+h_{1}\left[X_{m}\right] y_{j}+h_{2}\left[X_{m}\right] y_{j}^{2}+\cdots+h_{k}\left[X_{m}\right] y_{j}^{k}\right)=\sum_{\lambda: \lambda_{1} \leq k} h_{\lambda}\left[X_{m}\right] m_{\lambda}\left[Y_{m}\right]=\sum_{M}(\mathbf{x y})^{M}
$$


where the sum on the right hand side of the equation is over all $k$-bounded matrices $M=\left(m_{i j}\right)_{1 \leq i, j \leq n}$, whose entries are non-negative integers and satisfy $\sum_{i=1}^{n} m_{i j} \leq k$, and $(\mathbf{x y})^{M}=\prod_{i, j}\left(x_{i} y_{j}\right)^{m_{i j}}$.

In [81] the authors provide a bijection between the set of $k$-bounded matrices and pairs of tableaux $(P, Q)$ such that $P$ is a strong tableau and $Q$ is a weak tableau that are both of the same $(k+1)$-core shape. This is done by introducing an insertion algorithm which generalizes that of the RSK bijection (and reduces to RSK case when $k \geq \sum_{i, j} m_{i j}$ ).

Here we provide an exposition of a special case of this bijection, namely between permutation matrices (with entries in $\{0,1\}$ with a single 1 in each row and column) and pairs of tableaux $(P, Q)$, where $P$ is a strong standard tableau and $Q$ is a weak standard tableau.

The bijection in [81] (which is called 'affine insertion' in analogy with RSK-insertion) shows that the duality of the weak and strong functions can be expressed through the duality of the kernel in (6.6) and hence

$$
\prod_{j=1}^{m}\left(1+h_{1}\left[X_{m}\right] y_{j}+h_{2}\left[X_{m}\right] y_{j}^{2}+\cdots+h_{k}\left[X_{m}\right] y_{j}^{k}\right)=\sum_{\lambda: \lambda_{1} \leq k} \operatorname{Strong}_{\mathfrak{c}(\lambda)}\left[X_{m}\right] \operatorname{Weak}_{\mathfrak{c}(\lambda)}\left[Y_{m}\right]
$$

where the functions are defined as

$$
\operatorname{Weak}_{\kappa}\left[X_{m}\right]=\sum_{T} \mathbf{x}^{T}
$$

with the sum over all weak tableaux of shape $\kappa(\mathrm{a}(k+1)$-core $)$ and

$$
\operatorname{Strong}_{\kappa}\left[X_{m}\right]=\sum_{T} \mathbf{x}^{T}
$$

with the sum is over all strong tableaux of shape $\kappa$. In both cases $\mathbf{x}^{T}$ represents a monomial associated to the weight. The equality $\tilde{F}_{\lambda}^{(k)}\left[X_{m}\right]=\operatorname{Weak}_{\mathfrak{c}(\lambda)}\left[X_{m}\right]$ with dual $k$-Schur functions relies on a permutation action on the weight which can be found in [96]. The equality $s_{\lambda}^{(k)}\left[X_{m}\right]=\operatorname{Strong}_{\mathfrak{c}(\lambda)}\left[X_{m}\right]$ follows by a duality argument after showing that the functions Strong ${ }_{\kappa}\left[X_{m}\right]$ form a basis [81].

Let $f_{\kappa}^{\text {weak }}$ be the number of standard weak tableaux of shape $\kappa$ and $f_{\kappa}^{\text {strong }}$ be the number of standard strong tableaux of shape $\kappa$, where $\kappa$ is a $(k+1)$-core. If we take the coefficient of $x_{1} x_{2} \cdots x_{m} y_{1} y_{2} \cdots y_{m}$ in Equation (6.7), we find the following combinatorial result

$$
m !=\sum_{\lambda: \lambda_{1} \leq k} f_{\mathfrak{c}(\lambda)}^{\text {strong }} f_{\mathfrak{c}(\lambda)}^{\text {weak }}
$$

where the sum is over $k$-bounded partitions of $\lambda$ of $m$. This formula can be seen as a manifestation of a bijection between the set of permutations $\sigma$ of $S_{m}$ and pairs of tableaux $\left(P^{(k)}, Q^{(k)}\right)$, where $P^{(k)}$ is a strong standard tableau and $Q^{(k)}$ is a weak standard tableau. 


\subsection{A brief introduction to Fomin's growth diagrams}

Affine insertion is proved using Fomin's growth diagrams [42] which is a tool of presenting insertion algorithms on graded posets via certain local rules. To put the affine insertion algorithm in context, we give a brief presentation of the usual RSK algorithm between permutations and pairs of standard tableaux in terms of growth diagrams in order to show how the algorithms compare. In Section 6.3 we will demonstrate how the algorithm can be generalized to the bijection which explains Equation (6.10). The treatment we present in this section follows roughly the way of viewing Fomin's growth diagrams that is presented in [140, Section 5.2] with a few modifications in orientation.

We begin with an $n \times n$ permutation matrix corresponding to a permutation $\pi$ (we use the convention that row $i$ has a 1 in column $\pi_{i}$ ) and convert it into a pair of standard tableaux of the same shape. To do this we draw an $n \times n$ lattice of squares and label the vertices of this lattice with partitions and the centers of these squares with the entries of the permutation matrix. At the start of the procedure we begin by labeling only the first row and first column of vertices with empty partitions and fill in the rest of the diagram by a recursive procedure using a set of local rules.

To describe the RSK algorithm we describe a 'local rule' which is a bijection between two types of arrays.

\section{Case 1:}

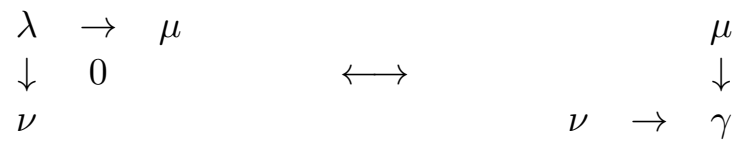

(a) If $\lambda=\mu=\nu$, then $\gamma=\nu$.

(b) If $\mu \neq \nu$, then $\gamma=\mu \cup \nu$.

(c) If $\lambda$ is strictly contained in $\mu=\nu$, then if $\mu$ is obtained from $\lambda$ by adding a cell in row $i$, then $\gamma$ is obtained from $\mu$ by adding a cell in row $i+1$.

\section{Case 2:}

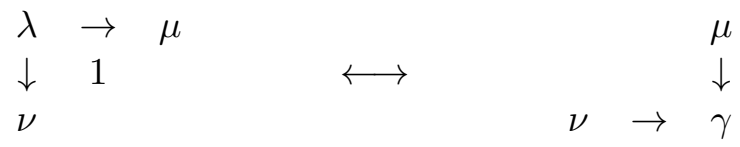

This case can only occur when $\lambda=\mu=\nu$, and then $\gamma$ is obtained from $\mu$ by adding a cell in the first row.

By successively applying these local rules, the growth diagram is filled in until it is an $(n+1) \times(n+1)$ array of partitions. Because each of the rules we apply is a bijection, we need only remember the last row and last column of the array and the rest of the table can be recovered by applying the local rule in reverse. The last row of this table is a sequence 
of partitions each of which differ by a single cell and so can be interpreted as a standard tableau which agrees with the insertion tableau of $\pi$. The last column of table is also a sequence of partitions each of which differ by a single cell; the corresponding standard tableau agrees with the recording tableau corresponding to the permutation $\pi$.

The important thing to notice is that the local rules can be reversed. For this reason if we are given just the pair of tableaux that represent the last row and the last column of the table, it is possible to reconstruct the entire table and hence the permutation matrix.

A beautiful feature of the growth diagram perspective on the RSK insertion algorithm is also that it makes it completely manifest that interchanging the insertion tableau $P$ and recording tableau $Q$ inverts the permutation $\pi$. This can be seen by interchanging the rows and columns of the array, which inverts the permutation matrix and interchanges $P$ and $Q$.

Example 6.1. Let us consider the permutation 4132 as a running example. We begin with a row and a column of 5 empty partitions and the entries of the permutation matrix in an array pictured below.

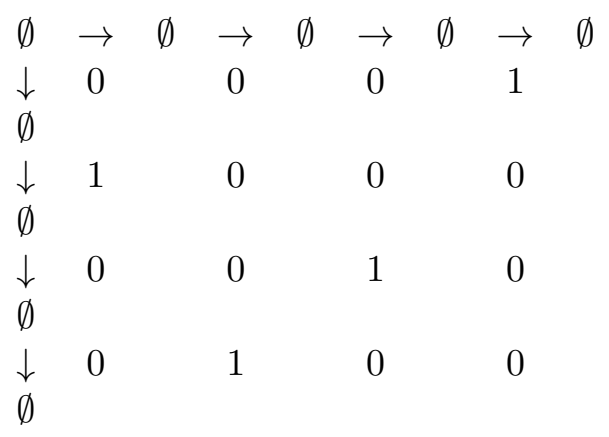

The local rules may be applied at first only in one place:

\begin{tabular}{|c|c|c|c|c|}
\hline$\emptyset \rightarrow$ & $\emptyset$ & $\rightarrow \emptyset$ & $\rightarrow \emptyset$ & $\rightarrow$ \\
\hline 0 & $\downarrow$ & 0 & 0 & 1 \\
\hline$\emptyset \rightarrow$ & $\emptyset$ & & & \\
\hline 1 & & 0 & 0 & 0 \\
\hline$\downarrow \quad 0$ & & 0 & 1 & 0 \\
\hline$\quad 0$ & & 1 & 0 & 0 \\
\hline
\end{tabular}

In successive steps, the local rules may be applied in each corner. 


$\begin{array}{lcccccccc}\emptyset & \rightarrow & \emptyset & \overrightarrow{0} & \emptyset & \overrightarrow{0} & \emptyset & \vec{G} & \emptyset \\ \downarrow & 0 & \downarrow & 0 & \downarrow & 0 & & 1 & \\ \emptyset & \rightarrow & \emptyset & \rightarrow & \emptyset & & & & \\ \downarrow & 1 & \downarrow & 0 & & 0 & & 0 & \\ \emptyset & \rightarrow & \square & & & & & & \\ \downarrow & 0 & & 0 & & 1 & & 0 & \\ \emptyset & & & & & & & & \\ \downarrow & 0 & & 1 & & 0 & & 0 & \\ \emptyset & & & & & & & & \\ & & & & & & & & \\ \emptyset & \rightarrow & \emptyset & \rightarrow & \emptyset & \rightarrow & \emptyset & \rightarrow & \emptyset \\ \downarrow & 0 & \downarrow & 0 & \downarrow & 0 & \downarrow & 1 & \\ \emptyset & \rightarrow & \emptyset & \rightarrow & \emptyset & \rightarrow & \emptyset & & \\ \downarrow & 1 & \downarrow & 0 & \downarrow & 0 & & 0 & \\ \emptyset & \rightarrow & \square & \rightarrow & \square & & & & \\ \downarrow & 0 & \downarrow & 0 & & 1 & & 0 & \\ \emptyset & \rightarrow & \square & & & & & & \\ \downarrow & 0 & & 1 & & 0 & & 0 & \\ \emptyset & & & & & & & & \end{array}$

By continuing to apply the local rules, we arrive at the following completed table:

$$
\begin{aligned}
& \emptyset \rightarrow \emptyset \rightarrow \emptyset \rightarrow \emptyset \rightarrow \emptyset \\
& \begin{array}{lllllllll}
\downarrow & 0 & \downarrow & 0 & \downarrow & 0 & \downarrow & 1 & \downarrow
\end{array} \\
& \emptyset \rightarrow \emptyset \rightarrow \emptyset \rightarrow \emptyset \rightarrow \square \\
& \downarrow \begin{array}{lllllllll} 
& 1 & \downarrow & 0 & \downarrow & 0 & \downarrow & 0 & \downarrow
\end{array} \\
& \emptyset \rightarrow \square \rightarrow \square \rightarrow \square \rightarrow \square Q \\
& \begin{array}{lllllllll}
\downarrow & 0 & \downarrow & 0 & \downarrow & 1 & \downarrow & 0 & \downarrow
\end{array} \\
& \begin{array}{ccccccccc}
\emptyset & \rightarrow & \square & \rightarrow & \square & \rightarrow & \square & \rightarrow & \square \\
\downarrow & 0 & \downarrow & 1 & \downarrow & 0 & \downarrow & 0 & \downarrow
\end{array} \\
& \emptyset \rightarrow \square \rightarrow \underset{P}{\square} \rightarrow \square \rightarrow \square
\end{aligned}
$$

Now in order to reconstruct this entire table, we need only remember the two standard tableaux $(P, Q)=\left(\frac{\frac{4}{3}}{\frac{1}{12}}, \frac{4}{2}, \frac{1}{13}\right)$ which correspond to the last row and the last column of the table. This indeed agrees with the usual insertion tableau $P$ and recording tableau $Q$ when row-inserting 4132 (see for example [140]). 


\subsection{Affine insertion}

Now that we have presented Fomin's growth diagrams as a tool for understanding RSK, we will give the local rules necessary to understand $k$-affine insertion for permutations. This is the algorithm presented in [81]. Parts of these local rules use operations which are described in Sections 1.2, 1.3 and 1.4 (in particular the action of $s_{i}$, the notion of strong and weak cover, and the vocabulary of content and residue).

We have stripped down the algorithm presented in [81] in hopes of making it clearer by having fewer details to follow. The algorithm presented in [81] is slightly more general because it includes an additional rule that generalizes the bijection from $k$-bounded nonnegative integer matrices to pairs tableaux of the same shape, the first is a strong semistandard tableau, the second is a weak semi-standard tableau. This more general bijection is sufficient to show Equation (6.7). Here we are pairing down the presentation rule to only demonstrate how Equation (6.10) works.

To do this we construct again a table that will be a growth diagram, where there is an $(n+1) \times(n+1)$ array of $(k+1)$-cores and between these entries we put the $n \times n$ permutation matrix.

There is an additional piece of information which is recorded in this matrix besides the shapes of the $k+1$-cores. The horizontal connectors in our growth diagrams keep track of (possibly empty) strong covers and the vertical connectors keep track of (possibly empty) weak covers. If there are two $(k+1)$-cores that are adjacent in the same row, $\tau \rightarrow \kappa$, then either $\tau=\kappa$ or $\kappa$ covers $\tau$ in the strong order. In the second case, we also need to mark one of the connected components of $\kappa / \tau$ in the diagram of $\kappa$ or keep track of this marking on the arrow $\stackrel{c}{\rightarrow}$, where $c$ represents the content of the diagonal of the marked cell. When working with the diagram in the examples below we only record this information by marking a cell of $\kappa / \tau$ within core $\kappa$ for compactness of notation.

We begin with the first row and column of this array consisting of empty cores only (and hence there are no markings necessary). Given a corner of the table that is partially filled in, we complete the rest with the following local rules.

Case 1:

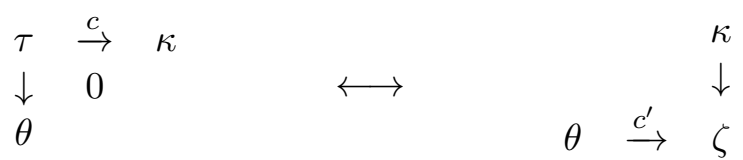

Try to apply (a)-(c) in this order. If a case does not apply, proceed to the next case.

(a) If $\tau=\kappa$, then $\zeta=\theta$ and neither $\kappa$ nor $\zeta$ will be marked; if $\tau=\theta$, then $\zeta=\kappa$ and $c^{\prime}=c$

(b) If $\kappa / \tau$ is not contained in $\theta / \tau$, let $r$ be the residue of the cells $\theta / \tau \quad(\bmod k+1)$ (there is exactly one). In this case $\zeta$ is $s_{r}$ applied to $\kappa$. One cell of $\kappa$ on the diagonal with content $c$ is marked. In $\zeta$ mark the component that has an overlap with the marked 
ribbon in $\kappa$ (alternatively, if $c$ does not have residue $r$, then $c^{\prime}=c$; otherwise $c^{\prime}$ is on one diagonal higher than $c$ ).

(c) If $\tau$ is strictly contained in $\kappa=\theta$, let $c^{\prime}$ be the content of the first diagonal which is weakly to the left of the marked ribbon of $\kappa / \tau$ and is an addable cell of $\kappa$. Then $\zeta=s_{c^{\prime}} \kappa$ and the marked cell of $\zeta$ is on the diagonal with content $c^{\prime}$.

\section{Case 2:}

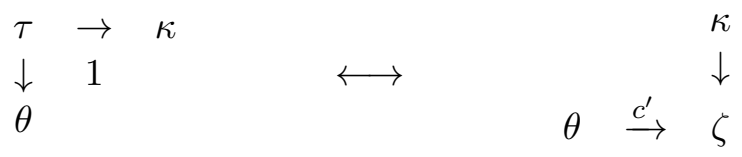

This case can only occur when $\tau=\kappa=\theta$, and then $\zeta$ is $s_{\tau_{1}}$ applied to $\tau$ (the effect of adding a cell in the first row of $\tau$, but as a $(k+1)$-core). A marking $c^{\prime}$ is added in last cell of the first row of $\zeta$.

Example 6.2. Let us compute the growth diagram for the matrix corresponding to the permutation 4132. As in Example 6.1, we begin our growth diagram with the first row and column consisting of empty cores.

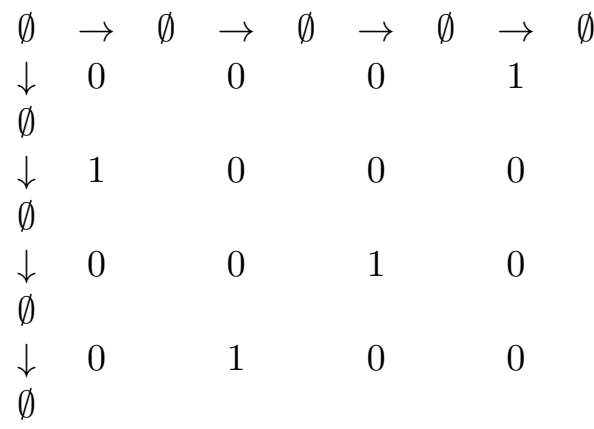

Below is the growth diagram for $k=1$ :

$$
\begin{array}{ccccccccc}
\emptyset & \rightarrow & \emptyset & \rightarrow & \emptyset & \rightarrow & \emptyset & \rightarrow & \emptyset \\
\downarrow & 0 & \downarrow & 0 & \downarrow & 0 & \downarrow & 1 & \downarrow \\
\emptyset & \rightarrow & \emptyset & \rightarrow & \emptyset & \rightarrow & \emptyset & \rightarrow & * \\
\downarrow & 1 & \downarrow & 0 & \downarrow & 0 & \downarrow & 0 & \downarrow \\
\emptyset & \rightarrow & * & \rightarrow & \square & \rightarrow & \square & \rightarrow & * \\
\downarrow & 0 & \downarrow & 0 & \downarrow & 1 & \downarrow & 0 & \downarrow \\
\emptyset & \rightarrow & * & \rightarrow & \square & \rightarrow & \square_{*} & \rightarrow & \square \\
\downarrow & 0 & \downarrow & 1 & \downarrow & 0 & \downarrow & 0 & \downarrow \\
\emptyset & \rightarrow & * & \rightarrow & \square_{*} & \rightarrow & \square_{*} & \rightarrow & \square^{*}
\end{array}
$$


Note that since the value of $k$ is too small, Case $1(\mathrm{~b})$ is not used. By reading the last row of this table we can encode it as a single strong tableau. When $k=1$ there is only one weak tableau of shape $(m, m-1, \ldots, 2,1)$. The last row and column of this table can then be encoded as

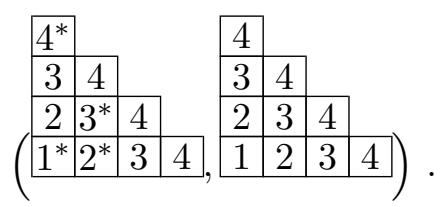

For $k=2$ and starting with the same permutation the situation is a little more complicated:

$$
\begin{array}{ccccccccc}
\emptyset & \rightarrow & \emptyset & \rightarrow & \emptyset & \rightarrow & \emptyset & \rightarrow & \emptyset \\
\downarrow & 0 & \downarrow & 0 & \downarrow & 0 & \downarrow & 1 & \downarrow \\
\emptyset & \rightarrow & \emptyset & \rightarrow & \emptyset & \rightarrow & \emptyset & \rightarrow & * \\
\downarrow & 1 & \downarrow & 0 & \downarrow & 0 & \downarrow & 0 & \downarrow \\
\emptyset & \rightarrow & * & \rightarrow & \square & \rightarrow & \square & \rightarrow & * \\
\downarrow & 0 & \downarrow & 0 & \downarrow & 1 & \downarrow & 0 & \downarrow \\
\emptyset & \rightarrow & * & \rightarrow & \square & \rightarrow & \square * & \rightarrow & * \\
\downarrow & 0 & \downarrow & 1 & \downarrow & 0 & \downarrow & 0 & \downarrow \\
\emptyset & \rightarrow & * & \rightarrow & \square * & \rightarrow & * & & * \\
\end{array}
$$

Now it is necessary to apply all four rules to fill in the growth diagram. The first time that Case 1 (b) occurs is constructing the last entry in the fourth row of cores (the last entry of the third row of the permutation matrix).

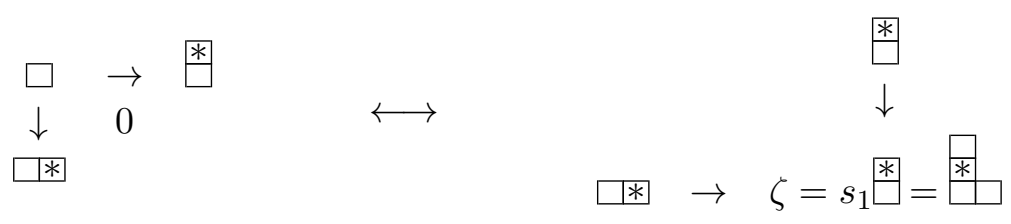

If we just record the last row as a strong tableau and the last column as a weak tableau, we have the follow pair:

$$
\begin{array}{|l|l|l|l|}
\hline 4^{*} & & 3 & \\
\hline 3^{*} & & & \\
\hline 1^{*} & 2^{*} & 3 \\
\hline & 1 & 3 & 4 \\
\hline
\end{array}
$$

and this pair of tableaux is sufficient to reconstruct the entire table.

For $k=3$, the case is similar to the $k=2$ case in that there are examples where all four rules are applied in order to construct the table: 


$$
\begin{array}{ccccccccc}
\emptyset & \rightarrow & \emptyset & \rightarrow & \emptyset & \rightarrow & \emptyset & \rightarrow & \emptyset \\
\downarrow & 0 & \downarrow & 0 & \downarrow & 0 & \downarrow & 1 & \downarrow \\
\emptyset & \rightarrow & \emptyset & \rightarrow & \emptyset & \rightarrow & \emptyset & \rightarrow & * \\
\downarrow & 1 & \downarrow & 0 & \downarrow & 0 & \downarrow & 0 & \downarrow \\
\emptyset & \rightarrow & * & \rightarrow & \square & \rightarrow & \square & \rightarrow & * \\
\downarrow & 0 & \downarrow & 0 & \downarrow & 1 & \downarrow & 0 & \downarrow \\
\emptyset & \rightarrow & * & \rightarrow & \square & \rightarrow & \square & \rightarrow & * \\
\downarrow & 0 & \downarrow & 1 & \downarrow & 0 & \downarrow & 0 & \downarrow \\
\emptyset & \rightarrow & * & \rightarrow & \square * & \rightarrow & * & \rightarrow & { }^{*}
\end{array}
$$

\begin{tabular}{|c|c|c|c|}
\hline$\sigma$ & $\left(P^{(1)}, Q^{(1)}\right)$ & $\left(P^{(2)}, Q^{(2)}\right)$ & $\left(P^{(\infty)}, Q^{(\infty)}\right)$ \\
\hline 123 & 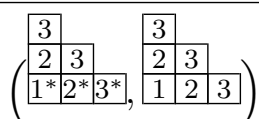 & 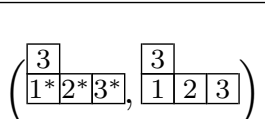 & $\left(\begin{array}{lll|l|l|}1^{*}\left|2^{*}\right| 3^{*} & 1 & 1 & 2 & 3 \\
\end{array}\right.$ \\
\hline 132 & \begin{tabular}{|l|l|l|l|l|}
$\begin{array}{l}3 \\
2\end{array}$ & $3^{*}$ \\
$1^{*}$ & $2^{*}$ & 3 \\
\end{tabular}, $\begin{array}{llll}3 & \\
2 & 3 & 3 & \\
1 & 2 & 3 \\
\end{array}$ & $\left(\begin{array}{l|l|l|l|}3^{*} & 3 & & \\
1^{*} 2^{*} \mid 3\end{array}, \begin{array}{l|l|l} & 2 & 3 \\
\end{array}\right)$ & 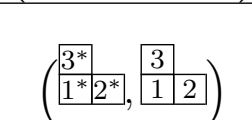 \\
\hline 213 & 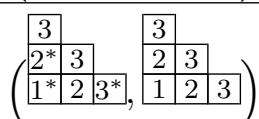 & $\left(\begin{array}{l|l|l} & \frac{3}{2^{*}} \\
1^{*} & 3^{*} & \frac{3}{2} \\
& \frac{1}{1} & 3 \\
\end{array}\right.$ & 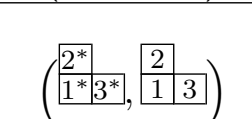 \\
\hline 231 & 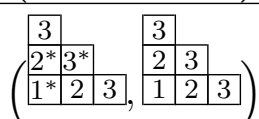 & 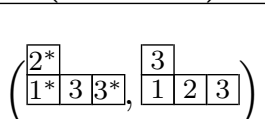 & 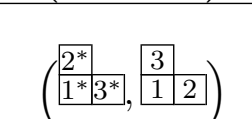 \\
\hline 312 & 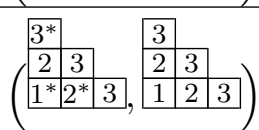 & 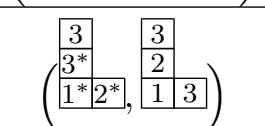 & 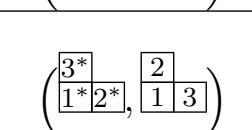 \\
\hline 321 & 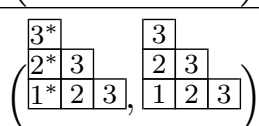 & 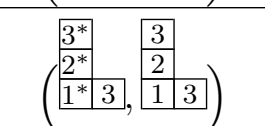 & $\left(\begin{array}{l|l}3^{*} & 3 \\
2^{*} & 2 \\
1^{*}, & 1 \\
\end{array}\right)$ \\
\hline
\end{tabular}

Because we can reconstruct the table from the last row and column of this table, it is necessary to keep track only of the strong tableau representing the last row and the weak tableau representing the last column. This is represented by the pair,

$$
\left.\begin{array}{|l|l|l|l|}
\hline 4^{*} & & & \\
\hline 3^{*} & & & \\
\hline 2 & & \\
\hline 1^{*} & 2^{*} & 4 \\
\hline & 1 & 3 & 4 \\
\hline
\end{array}\right) .
$$

Example 6.3. In the following table we have presented permutations of 3 (corresponding to permutation matrices) which are in bijection with pairs of weak and strong tableaux for $k=1,2$ and 3 . When $k=3$, the tableaux are in bijection with pairs of standard tableaux of the same shape by dropping the markings in the strong tableaux. 
Example 6.4. In the following table we have permutations of 4 (corresponding to permutation matrices) which are in bijection with pairs of weak and strong tableaux for $k=1,2$ and 3,4 . When $k=4$, the tableaux are in bijection with pairs of standard tableaux of the same shape by dropping the markings in the strong tableaux. 


\begin{tabular}{|c|c|c|c|c|}
\hline$\sigma$ & $\left(P^{(1)}, Q^{(1)}\right)$ & $\left(P^{(2)}, Q^{(2)}\right)$ & $\left(P^{(3)}, Q^{(3)}\right)$ & $\left(P^{(\infty)}, Q^{(\infty)}\right)$ \\
\hline 1234 & 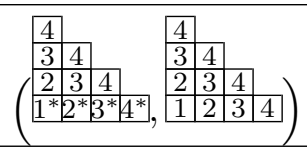 & 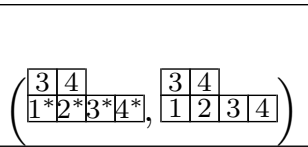 & 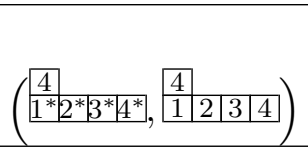 & 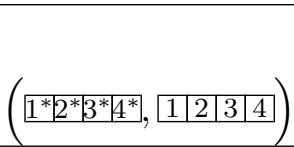 \\
\hline 1243 & 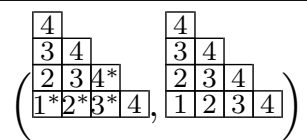 & 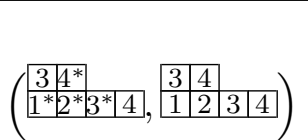 & 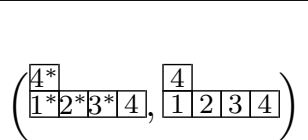 & $\left(\frac{4^{*}}{1^{*} 2^{*} 3^{*}}\right.$, \\
\hline 1324 & 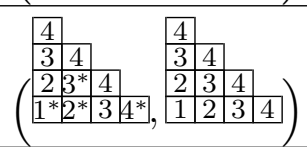 & 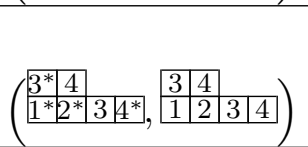 & 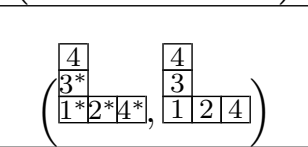 & $14)$ \\
\hline 1342 & 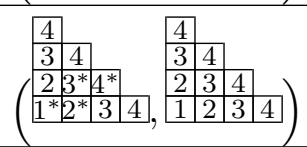 & 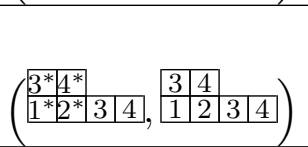 & 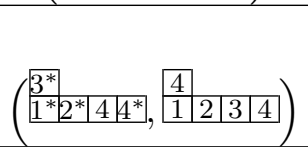 & $\left(\frac{3^{*}}{1^{*} 2^{*} 1^{*},}\right.$, \\
\hline 1423 & 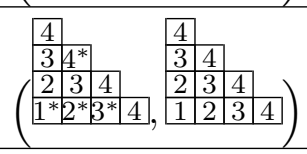 & 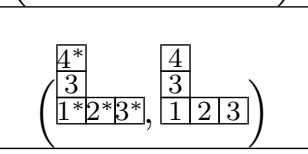 & $2 \mid 4)$ & \\
\hline 1432 & 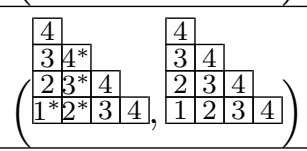 & 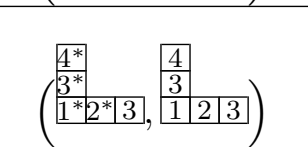 & 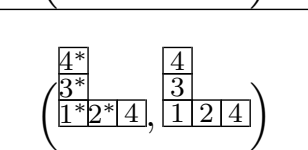 & 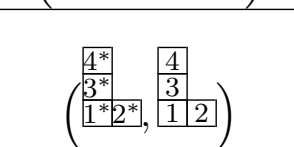 \\
\hline 2134 & 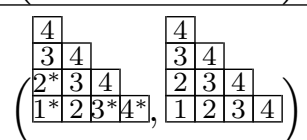 & 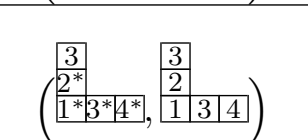 & 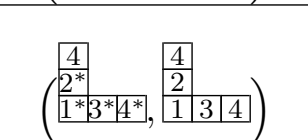 & $\left(\frac{2}{1}\right.$ \\
\hline 2143 & 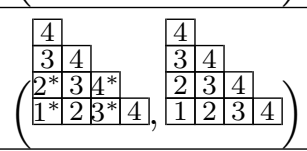 & 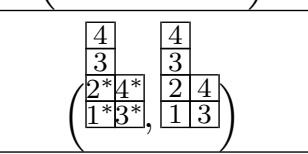 & & \\
\hline 2314 & 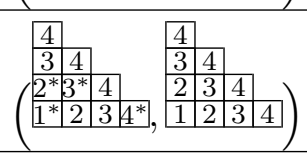 & 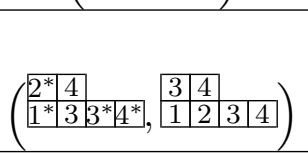 & 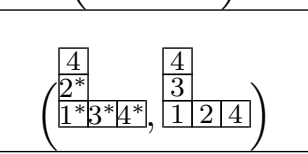 & 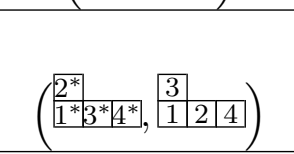 \\
\hline 2341 & 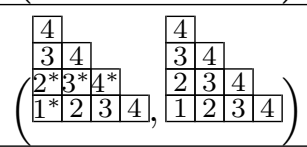 & 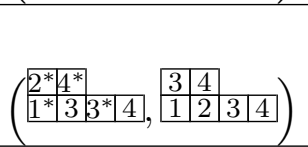 & 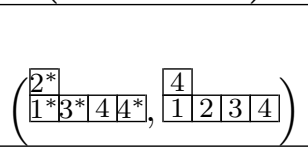 & 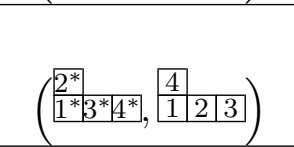 \\
\hline 2413 & 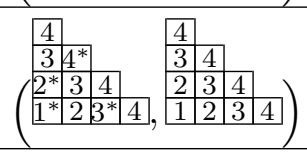 & 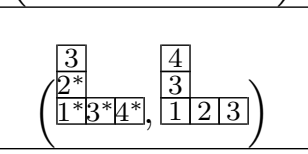 & 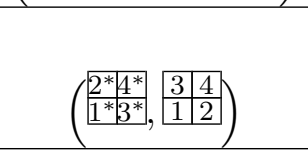 & $\left(\begin{array}{ll|l}* 4^{*} * & 3 & 4 \\
1 * 3 * & 1 & 2 \\
\end{array}\right)$ \\
\hline 2431 & 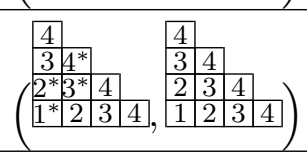 & 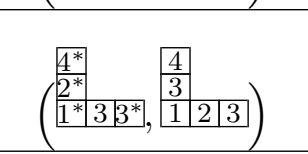 & 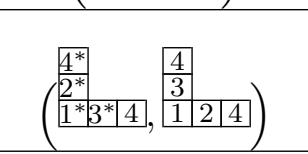 & $\left(\begin{array}{l|l|l}* * \\
2^{*} \\
1^{*} 3^{*}\end{array}, \begin{array}{|l|l} & \\
3 & \\
1 & 2\end{array}\right)$ \\
\hline
\end{tabular}




\begin{tabular}{|c|c|c|c|c|}
\hline$\sigma$ & $\left(P^{(1)}, Q^{(1)}\right)$ & $\left(P^{(2)}, Q^{(2)}\right)$ & $\left(P^{(3)}, Q^{(3)}\right)$ & $\left(P^{(\infty)}, Q^{(\infty)}\right)$ \\
\hline 3124 & 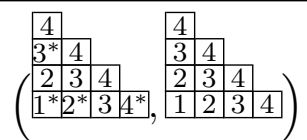 & 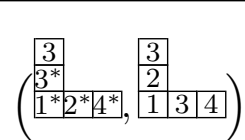 & 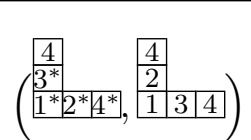 & 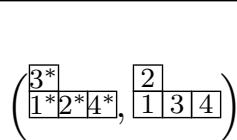 \\
\hline 3142 & 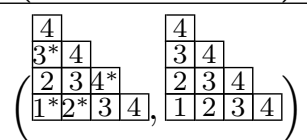 & 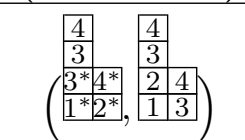 & $\begin{array}{l}3^{*} 4^{*} \\
I^{*} 2^{*} \\
\end{array}$ & ]) \\
\hline 3214 & 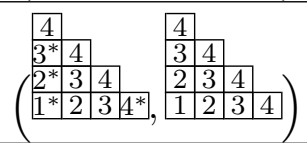 & 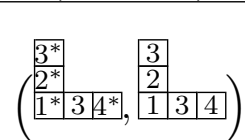 & $\left.\begin{array}{ll}\frac{4}{3^{*}} & \frac{4}{3} \\
\left(\frac{2^{*}}{1^{*}} 4^{*},\right. & \frac{1}{2} \\
& \frac{1}{4} \\
\end{array}\right)$ & 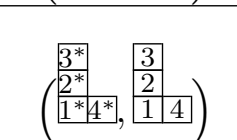 \\
\hline 3241 & 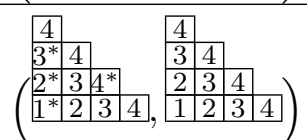 & $\rightarrow$ & 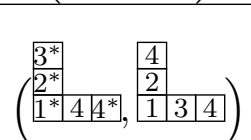 & 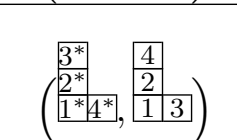 \\
\hline 3412 & 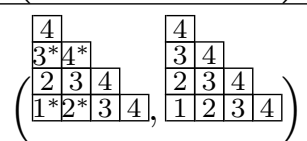 & 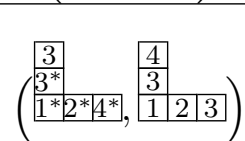 & - & \\
\hline 3421 & 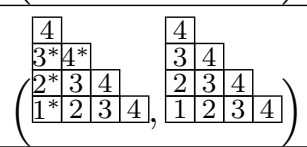 & 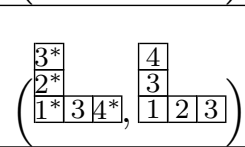 & 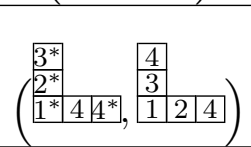 & 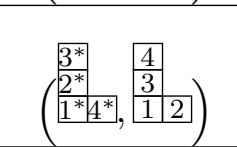 \\
\hline 4123 & 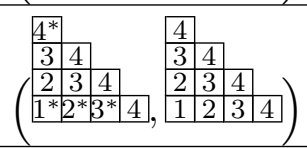 & 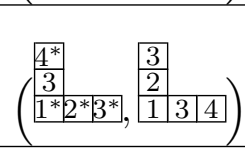 & 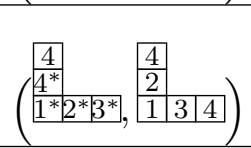 & 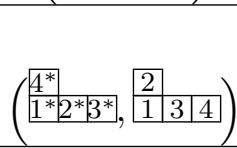 \\
\hline 4132 & 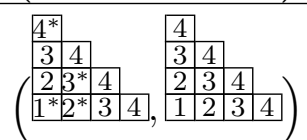 & 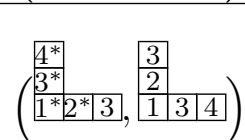 & 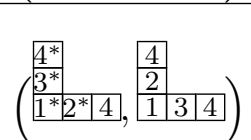 & $\left(\begin{array}{l|l}\frac{4^{*}}{3^{*}} & \frac{4}{2} \\
1^{*} 2^{*} & \frac{1}{1} \\
\end{array}\right)$ \\
\hline 4213 & 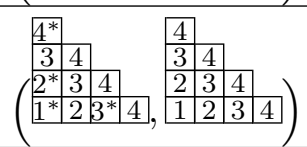 & 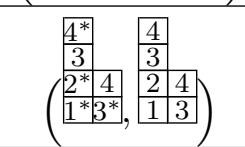 & 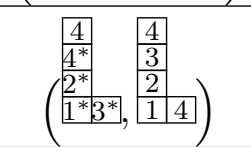 & 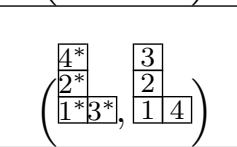 \\
\hline 4231 & 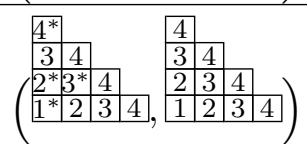 & 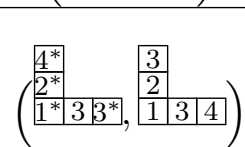 & 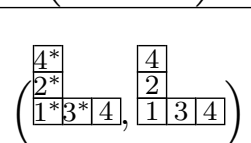 & $\left(\begin{array}{l|l}\frac{4^{*}}{2^{*}} & \left.\begin{array}{ll}4 \\
1^{*} 3^{*}\end{array}, \begin{array}{ll}2 \\
1\end{array}\right) \\
\end{array}\right)$ \\
\hline 4312 & 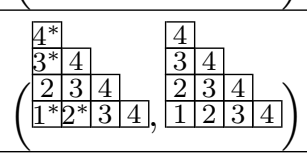 & 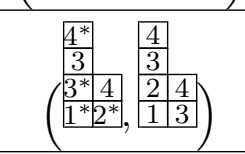 & 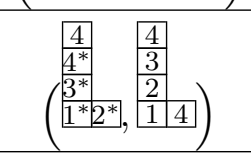 & $\left(\begin{array}{lll}4^{*} & \begin{array}{ll}3 * \\
1^{*} 2^{*}\end{array}, \begin{array}{ll}3 \\
2 \\
1\end{array} \\
\end{array}\right.$ \\
\hline 4321 & 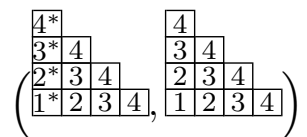 & 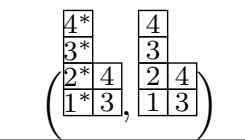 & 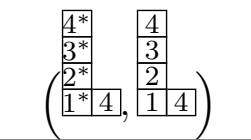 & 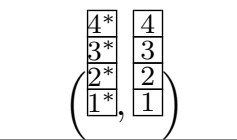 \\
\hline
\end{tabular}




\subsection{The $t$-compatible affine insertion algorithm}

Since the expression on the left of Equation (6.10) is independent of $k$, we realize that since $w \leftrightarrow\left(P^{(k)}, Q^{(k)}\right) \leftrightarrow\left(P^{(k+1)}, Q^{(k+1)}\right)$, where $P^{(k)}$ and $P^{(k+1)}$ are strong $k$ and $(k+1)$ tableaux may help us to see how $s_{\lambda}^{(k)}$ can be expressed as a positive sum of elements $s_{\mu}^{(k+1)}$. It may be possible to understand the expansion of $s_{\lambda}^{(k)}$ in terms of Schur functions or in terms of $s_{\lambda}^{(k+1)}$ using this bijection, but this would impose certain conditions on the shapes of tableaux $P^{(k)}$ and $P^{(k+1)}$ that do not seem to hold.

In this section we present some evidence suggesting that one might hope for an affine insertion bijection which has additional properties that are not shared with the affine insertion algorithm of Section 6.3.

There is also a $t$-analogue of Equation (6.7) which can be used as a stronger guide for the combinatorics of an analogue of the Robinson-Schensted-Knuth bijection. The coefficient of $m_{\left(1^{m}\right)}[X]$ in $Q_{\left(1^{m}\right)}^{\prime}[X ; t]$ is equal to the $t$-analogue of $m !,[m]_{t} !=(1-t)^{-m} \prod_{i=1}^{m}\left(1-t^{i}\right)$, and hence we have

$$
\begin{aligned}
{[m]_{t} ! } & =\left\langle Q_{\left(1^{m}\right)}^{\prime}[X ; t], h_{\left(1^{m}\right)}[X]\right\rangle=\sum_{\lambda \vdash m} K_{\lambda\left(1^{m}\right)}(t)\left\langle s_{\lambda}[X], h_{\left(1^{m}\right)}[X]\right\rangle \\
& =\sum_{\lambda \vdash m} K_{\lambda\left(1^{m}\right)}(t) f_{\lambda}=\sum_{\lambda: \lambda_{1} \leq k} K_{\lambda\left(1^{m}\right)}^{(k)}(t)\left\langle s_{\lambda}^{(k)}[X ; t], h_{\left(1^{m}\right)}[X]\right\rangle .
\end{aligned}
$$

The polynomial $\left\langle s_{\lambda}^{(k)}[X ; t], h_{\left(1^{m}\right)}[X]\right\rangle$ is equal to $\sum_{P} t^{\operatorname{spin}(P)}$, where the sum is over all strong standard $k$-tableaux of shape $\mathfrak{c}(\lambda)$ by Equation (3.16). The coefficient $K_{\lambda_{\left(1^{m}\right)}}^{(k)}(t)$ is a $t$-analogue of the number of standard weak $k$-tableaux of shape $\mathfrak{c}(\lambda)$. This equation is just one possible refinement of Equation (6.10). Similarly, the right hand side of this equation depends on $k$ while the left hand side does not and this indicates that we might hope to see some relationship between the bijection at level $k$ and level $k+1$ that relates the $t$ statistic.

If we are looking to explain this algebraic expression with a bijection, we would like to find a statistic charge on standard weak $k$-tableaux and a bijection between permutations and pairs of strong and weak tableaux of the same shape $w^{-1} \leftrightarrow\left(P^{(k)}, Q^{(k)}\right)$ such that

$$
\operatorname{charge}(w)=\operatorname{spin}\left(P^{(k)}\right)+\operatorname{charge}\left(Q^{(k)}\right) .
$$

Note that we taking the association with $w^{-1}$ to ensure that everything agrees since as $k \rightarrow \infty$ the statistic charge was defined so that the charge of a permutation is the charge of its insertion tableau. The statistic spin on $k$-strong tableaux is different in nature than the charge statistic and in general $\operatorname{spin}\left(P^{(\infty)}\right)=0$.

The reason the previous affine insertion algorithm is not quite 'real' is that we are unable to use it to explain this $t$-analogue. A 'real' affine insertion algorithm would allow us to take a definition of the charge statistic so that if $w^{-1} \leftrightarrow\left(P^{(k)}, Q^{(k)}\right)$, then charge $\left(Q^{(k)}\right)=$ 
$\operatorname{charge}(w)-\operatorname{spin}\left(P^{(k)}\right)$. It would need to be the case that if $u$ and $v$ are two different permutations such that $u^{-1} \leftrightarrow\left(P^{1(k)}, Q^{(k)}\right)$ and $v^{-1} \leftrightarrow\left(P^{2(k)}, Q^{(k)}\right)$, then charge $(u)-$ $\operatorname{spin}\left(P^{1(k)}\right)=\operatorname{charge}(v)-\operatorname{spin}\left(P^{2(k)}\right)$. The following example, based on the calculations from Example 6.3, shows that this affine insertion algorithm is not compatible with the spin statistic in this sense.

Example 6.5. It is probably easiest to see that Equation (6.12) cannot hold in our example unless charge $(w)=\operatorname{spin}\left(P^{(1)}\right)$ because when $k=1$ all of the $Q^{(1)}$ tableaux are the same.

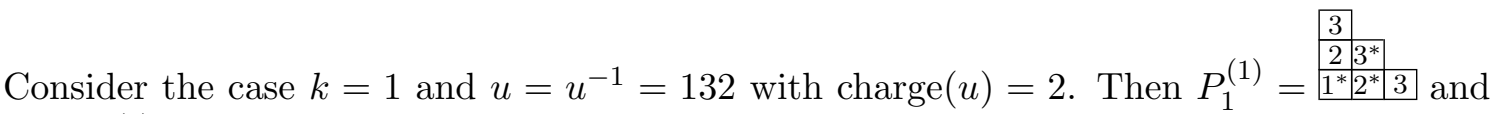
$\operatorname{spin}\left(P_{1}^{(1)}\right)=1$.

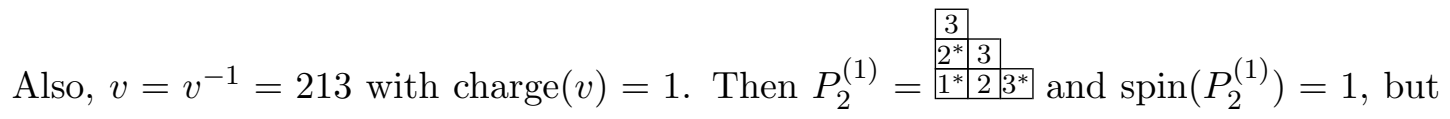
$Q^{(1)}=$\begin{tabular}{lll}
3 & 3 \\
\hline & 3 & 2 \\
1 & 2 & 3
\end{tabular} is the same in both cases and if there is a charge statistic it should be the same.

\section{The $k$-shape poset and a branching rule for expressing $k$-Schur in $(k+1)$-Schur functions}

One of the more recent developments with the definition of $k$-Schur functions defined as the sum over strong tableaux is an explanation of why they expand positively in the $(k+1)$ Schur functions. That is, there are nonnegative integer coefficients $b_{\mu \lambda}^{(k)}$ such that

$$
s_{\mu}^{(k-1)}=\sum_{\lambda} b_{\mu \lambda}^{(k)} s_{\lambda}^{(k)}
$$

and in this section we will describe a combinatorial interpretation for the coefficients $b_{\mu \lambda}^{(k)}$.

If we consider a partition $\lambda$ as a collection of cells, then $\operatorname{Int}^{k}(\lambda)=\{b \in \operatorname{dg}(\lambda)$ : $\left.\operatorname{hook}_{\lambda}(b)>k\right\}$ and $\partial^{k}(\lambda)=\lambda / \operatorname{Int}^{k}(\lambda)$. We define the row shape (resp. column shape) of $\lambda$ to be the composition $r s^{k}(\lambda)$ (resp. $c s^{k}(\lambda)$ ) consisting of the number of cells in each of the rows of $\partial^{k}(\lambda)$. The partition $\lambda$ is said to be a $k$-shape if both $r s^{k}(\lambda)$ and $c s^{k}(\lambda)$ are partitions. Let $\Pi^{k}$ denote the set of $k$-shapes and $\Pi_{N}^{k}$ represent the set of $k$-shapes $\lambda$ such that $\left|\partial^{k}(\lambda)\right|=N$. Notice that both the $k$-cores and the $(k+1)$-cores of size $N$ are a subset of $\Pi_{N}^{k}$.

Example 7.1. If $k=3$, then

$$
\Pi_{3}^{3}=\{(1,1,1),(2,1),(3),(3,1),(2,1,1)\}
$$


since they correspond to the 3-boundaries

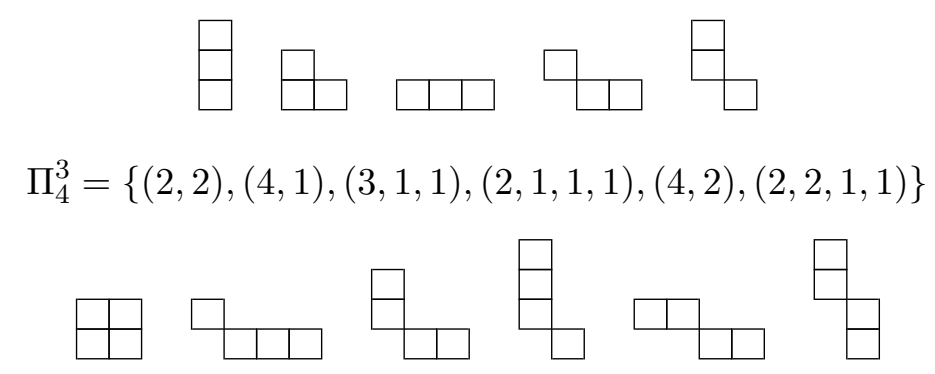

Example 7.2. The partition $\lambda=(6,2,1)$ is a 4 -shape but it is not a 3 -shape. We calculate that

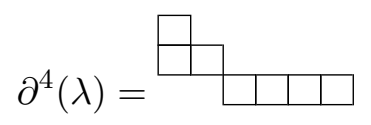

and hence $r s^{4}(\lambda)=(4,2,1)$ and $c s^{4}(\lambda)=(2,1,1,1,1,1)$, but

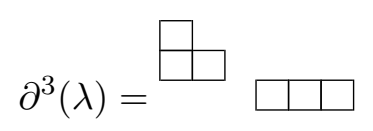

so that $r s^{3}(\lambda)=(3,2,1)$ and $c s^{3}(\lambda)=(2,1,0,1,1,1)$ and hence it is not a 3 -shape.

Example 7.3. We include a table of the number of $k$-shapes for $1 \leq k \leq 9$ and $1 \leq N \leq 13$. In the limit (for $N<k$ ) it is the case that $\left|\Pi_{N}^{k}\right|$ is equal to the number of partitions of $N$.

\begin{tabular}{|c||c|c|c|c|c|c|c|c|c|c|c|c|c|c|}
\hline$k \backslash N$ & 0 & 1 & 2 & 3 & 4 & 5 & 6 & 7 & 8 & 9 & 10 & 11 & 12 & 13 \\
\hline$k=1$ & 1 & 1 & 1 & 1 & 1 & 1 & 1 & 1 & 1 & 1 & 1 & 1 & 1 & 1 \\
\hline$k=2$ & 1 & 1 & 3 & 3 & 6 & 6 & 10 & 10 & 15 & 15 & 21 & 21 & 28 & 28 \\
\hline$k=3$ & 1 & 1 & 2 & 5 & 6 & 10 & 15 & 21 & 27 & 40 & 48 & 65 & 81 & 103 \\
\hline$k=4$ & 1 & 1 & 2 & 3 & 8 & 9 & 15 & 23 & 35 & 42 & 69 & 86 & 116 & 155 \\
\hline$k=5$ & 1 & 1 & 2 & 3 & 5 & 11 & 14 & 21 & 30 & 49 & 67 & 90 & 120 & 177 \\
\hline$k=6$ & 1 & 1 & 2 & 3 & 5 & 7 & 16 & 19 & 30 & 41 & 60 & 89 & 127 & 163 \\
\hline$k=7$ & 1 & 1 & 2 & 3 & 5 & 7 & 11 & 21 & 27 & 40 & 56 & 79 & 107 & 163 \\
\hline$k=8$ & 1 & 1 & 2 & 3 & 5 & 7 & 11 & 15 & 29 & 36 & 54 & 73 & 105 & 138 \\
\hline$k=9$ & 1 & 1 & 2 & 3 & 5 & 7 & 11 & 15 & 22 & 38 & 49 & 70 & 97 & 134 \\
\hline
\end{tabular}

We will define a poset structure on the set $\Pi_{N}^{k}$ by describing how the elements are related by a set of row and column moves. In order to define row and column moves we need to define a notion of row-type and column-type strings which describe the movement of cells to get from one $k$-shape to another.

For a cell $b=(x, y)$ we say that the diagonal index of $b$ is $d(b)=y-x$. Two cells $b, b^{\prime}$ are called contiguous if $\left|d(b)-d\left(b^{\prime}\right)\right| \in\{k, k+1\}$. A string of length $\ell$ is a skew shape $\mu / \lambda$ 
consisting of cells $\left\{a_{1}, a_{2}, \ldots, a_{\ell}\right\}$ such that $a_{i+1}$ and $a_{i}$ are contiguous for each $1 \leq i<\ell$ and $a_{i+1}$ lies strictly below $a_{i}$.

For a skew diagram $D$, define $\operatorname{left}_{a}(D)$ to be the leftmost cell in the same row as the cell $a$ and $\operatorname{bot}_{a}(D)$ to be the bottommost cell in same column as $a$.

A string $\mu / \lambda=\left\{a_{1}, a_{2}, \ldots, a_{\ell}\right\}$ is called a row-string if $\operatorname{hook}_{\lambda}\left(\operatorname{left}_{a_{1}}\left(\partial^{k}(\lambda)\right)\right)=k$ and $\operatorname{hook}_{\lambda}\left(\operatorname{bot}_{a_{\ell}}\left(\partial^{k}(\lambda)\right)\right)<k$. It is called a column-string if the transpose picture is a rowstring, or, if hook ${ }_{\lambda}\left(\operatorname{left}_{a_{1}}\left(\partial^{k}(\lambda)\right)\right)<k$ and $\operatorname{hook}_{\lambda}\left(\operatorname{bot}_{a_{\ell}}\left(\partial^{k}(\lambda)\right)\right)=k$.

A row move (resp. column move) of rank $r$ and length $\ell$ is a chain of parititions $\lambda=\lambda^{0} \subset \lambda^{1} \subset \cdots \subset \lambda^{r}=\mu$ that satisfies

- $\lambda, \mu \in \Pi^{k}$

- $s_{i}=\lambda^{i} / \lambda^{i-1}$ is a row-type (resp. column-type) string consisting of $\ell$ cells for all $1 \leq i \leq r$.

- the strings $s_{i}$ are all translates of each other

- the top cells (resp. rightmost cells) of $s_{1}, s_{2}, \ldots, s_{r}$ occur in consecutive columns (resp. rows) from left to right (resp. bottom to top).

To be clear, a column move is a sequence of partitions whose conjugate partitions are a row move.

Example 7.4. If $k=5$ and $N=18$, then $\lambda=(9,5,4,4,2,1,1,1)$ and $\mu=(9,7,5,4,2,1,1,1)$ are both 5 -shapes and the sequence of partitions $\lambda^{0}=\lambda \subset \lambda^{1}=(9,5,5,4,2,1,1,1) \subset \lambda^{2}=$ $(9,6,5,4,2,1,1,1) \subset \lambda^{3}=\mu$ have the following boundaries:
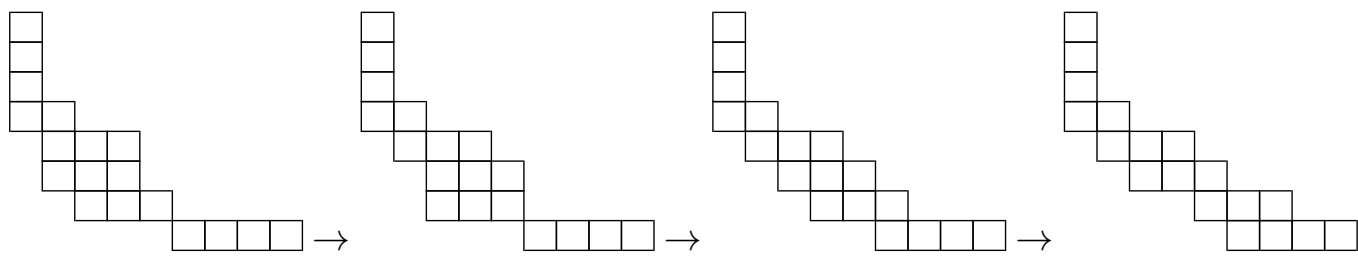

The set $\Pi_{N}^{k}$ is endowed with a poset structure with an edge from $\lambda$ to $\mu$ (or a cover relation $\lambda \rightarrow \mu$ ) if there is a row or a column move from $\lambda$ to $\mu$. With some analysis one can show that the minimal elements of the poset structure on $\Pi_{N}^{k}$ are the $k$-cores and the maximal elements are the $(k+1)$-cores.

Example 7.5. The set of elements $\Pi_{5}^{3}$ is endowed with the the following poset structure. The edges representing a row move are labeled with an $r$ and those with a column move with a $c$. 


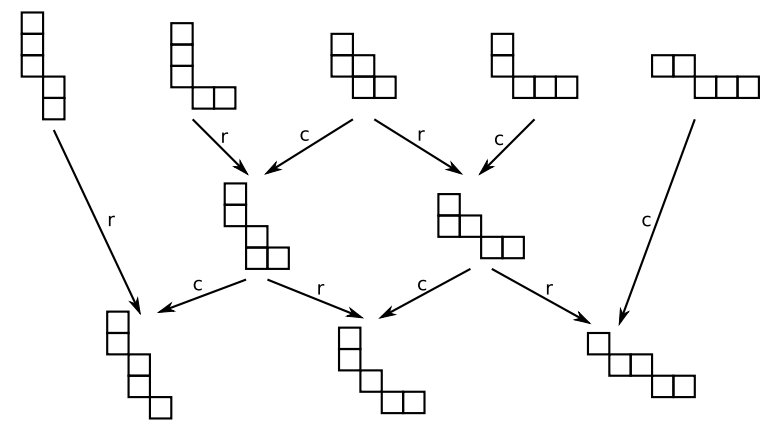

Example 7.6. In the set of elements of $\Pi_{5}^{2}$, we see that the highest elements in this partial order are those that are the lowest elements of $\Pi_{5}^{3}$. The Hasse diagram resembles the following.

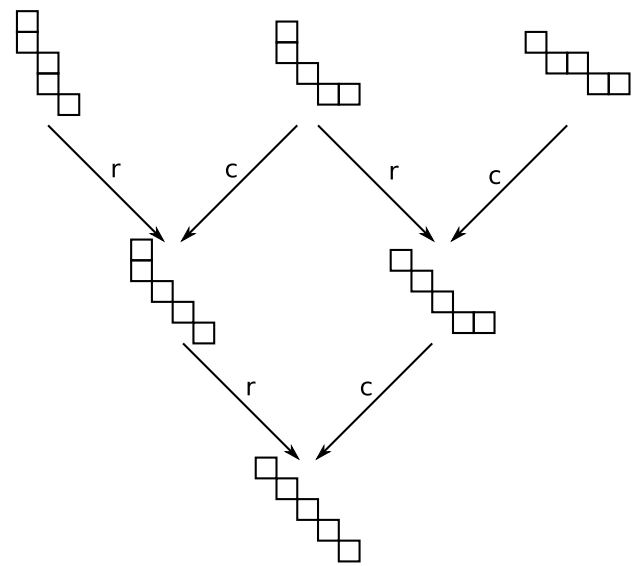

Now the combinatorial interpretation for the branching coefficients of the $k$-Schur functions is given in terms of paths within this poset (up to an equivalence on diamonds).

Define a charge of a move to be 0 if it is a row move and $r \ell$ for a move of length $\ell$ and rank $r$.

If $m, M, \tilde{m}, \tilde{M}$ are moves relating the $k$-shapes $\lambda$ and $\gamma$ through the following diagram,

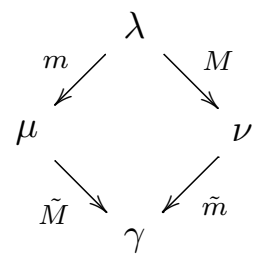

such that

$$
\operatorname{charge}(m)+\operatorname{charge}(\tilde{M})=\operatorname{charge}(\tilde{m})+\operatorname{charge}(M)
$$


then the two paths from $\lambda$ to $\gamma$ are equivalent. Now consider two $k$-shapes $\kappa, \tau \in \Pi_{N}^{k}$ where $\kappa$ is a $(k+1)$-core and $\tau$ is a $k$-core. Let $\mathcal{P}(\kappa, \tau)$ be the set of paths from $\kappa$ to $\tau$ with respect to this equivalence relation.

The reason the $k$-shapes are related to $k$-Schur functions is that we have the following theorem.

Theorem 7.7. [82, Theorem 2] Let $\lambda$ be $k-1$ bounded partition,

$$
s_{\lambda}^{(k-1)}[X]=\sum_{\mu}\left|\mathcal{P}\left(\mathfrak{c}_{k}(\mu), \mathfrak{c}_{k-1}(\lambda)\right)\right| s_{\mu}^{(k)}[X]
$$

where the sum is over all $k$-bounded partitions $\mu$.

While there is not a complete proof, the charge is defined so that the following conjecture should also hold.

Conjecture 7.8. [82, Conjecture 3] Let $\lambda$ be $k-1$ bounded partition,

$$
s_{\lambda}^{(k-1)}[X ; t]=\sum_{\mu} \sum_{\mathbf{p} \in \mathcal{P}\left(\mathfrak{c}_{k+1}(\mu), \mathfrak{c}_{k}(\lambda)\right)} t^{\operatorname{charge}(\mathbf{p})} s_{\mu}^{(k)}[X ; t]
$$

where the sum is over all $k$-bounded partitions $\mu$.

Example 7.9. Example 7.5 and Theorem 7.7 can be used to calculate the following expansions of 2-Schur functions in 3-Schur functions.

$$
\begin{gathered}
s_{11111}^{(2)}[X]=s_{11111}^{(3)}[X]+s_{2111}^{(3)}[X]+s_{221}^{(3)}[X] \\
s_{2111}^{(2)}[X]=s_{2111}^{(3)}[X]+s_{221}^{(3)}[X]+s_{311}^{(3)}[X] \\
s_{221}^{(2)}[X]=s_{221}^{(3)}[X]+s_{311}^{(3)}[X]+s_{32}^{(3)}[X] .
\end{gathered}
$$

This is because the two paths from the shape $(3,2,1)$ to $(4,2,1,1)$ are equivalent under the diamond relation.

The relation that appears in Conjecture 7.8 says that

$$
\begin{aligned}
s_{11111}^{(2)}[X ; t] & =s_{11111}^{(3)}[X ; t]+t^{2} s_{2111}^{(3)}[X ; t]+t^{3} s_{221}^{(3)}[X ; t] \\
s_{2111}^{(2)}[X ; t] & =s_{2111}^{(3)}[X ; t]+t s_{221}^{(3)}[X ; t]+t^{2} s_{311}^{(3)}[X ; t] \\
s_{221}^{(2)}[X ; t] & =s_{221}^{(3)}[X ; t]+t s_{311}^{(3)}[X ; t]+t^{2} s_{32}^{(3)}[X ; t] .
\end{aligned}
$$

This is because the column moves with charge 2 are those from $(3,2,1,1)$ to $(3,2,2,1,1)$ and from $(5,2)$ to $(5,3,1)$. The others all have charge 1 . 
Example 7.10. The other poset we have drawn shows that $s_{11111}^{(1)}[X]=s_{11111}^{(2)}[X]+$ $2 s_{2111}^{(2)}[X]+s_{221}^{(2)}[X]$. In this example the two paths from $(2,1,1,1)$ to $(1,1,1,1,1)$ are not equivalent. We can check that the charge of the move from $(4,2,1,1)$ to $(4,3,2,1,1)$ is 3 , while the move from $(5,3,2,1)$ to $(5,4,3,2,1)$ is 4 . Since the charge from $(5,3,1)$ to $(5,3,2,1)$ is 2 , we conclude that Conjecture 7.8 can be used to compute

$$
s_{11111}^{(1)}[X ; t]=s_{11111}^{(2)}[X ; t]+\left(t^{3}+t^{4}\right) s_{2111}^{(2)}[X ; t]+t^{6} s_{221}^{(2)}[X ; t] .
$$




\title{
Chapter 3
}

\section{Stanley symmetric functions and Peterson algebras}

\author{
THOMAS LAM ${ }^{1}$ \\ tfylam@umich.edu
}

This purpose of this chapter is to introduce Stanley symmetric functions and affine Stanley symmetric functions from the combinatorial and algebraic point of view. The presentation roughly follows 3 lectures I gave at a conference titled "Affine Schubert Calculus" held in July of 2010 at the Fields Institute in Toronto ${ }^{2}$.

The goal is to develop the theory (with the exception of positivity) without appealing to geometric reasoning. The material is aimed at an audience with some familiarity with symmetric functions, Young tableaux and Coxeter groups/root systems.

Stanley symmetric functions are a family $\left\{F_{w} \mid w \in S_{n}\right\}$ of symmetric functions indexed by permutations. They were invented by Stanley [150] to enumerate the reduced words of elements of the symmetric group. The most important properties of the Stanley symmetric functions are their symmetry, established by Stanley, and their Schur positivity, first proven by Edelman and Greene [40], and by Lascoux and Schützenberger [108].

Recently, a generalization of Stanley symmetric functions to affine permutations was developed in [78]. These affine Stanley symmetric functions turned out to have a natural geometric interpretation [79]: they are pullbacks of the cohomology Schubert classes of the affine flag variety $L S U(n) / T$ to the affine Grassmannian (or based loop space) $\Omega S U(n)$ under the natural map $\Omega S U(n) \rightarrow L S U(n) / T$. The combinatorics of reduced words and the geometry of the affine homogeneous spaces are connected via the nilHecke ring of Kostant and Kumar [75], together with a remarkable commutative subalgebra due to Peterson [131].

\footnotetext{
${ }^{1}$ The author was supported by NSF grants DMS-0652641, DMS-0901111, and DMS-1160726, and by a Sloan Fellowship.

${ }^{2}$ see http://www.fields.utoronto.ca/programs/scientific/10-11/schubert/
} 
The symmetry of affine Stanley symmetric functions follows from the commutativity of Peterson's subalgebra, and the positivity in terms of affine Schur functions is established via the relationship between affine Schubert calculus and quantum Schubert calculus $[88,116]$. The affine-quantum connection was also discovered by Peterson.

The affine generalization also connects Stanley symmetric functions with the theory of Macdonald polynomials [120] and $k$-Schur functions [92] - my own involvement in this subject began when I heard a conjecture of Mark Shimozono relating the Lapointe-LascouxMorse $k$-Schur functions to the affine Grassmannian.

While the definition of (affine) Stanley symmetric functions does not easily generalize to other (affine) Weyl groups (see [19, 20, 46, 84, 132]), the algebraic and geometric constructions mentioned above do.

The first third (Sections 1 - 3) of the chapter centers on the combinatorics of reduced words. We discuss reduced words in the (affine) symmetric group, the definition of the (affine) Stanley symmetric functions, and introduce the Edelman-Greene correspondence. Section 4 reviews the basic notation of Weyl groups and affine Weyl groups. In Sections 5-9 we introduce and study four algebras: the nilCoxeter algebra, the Kostant-Kumar nilHecke ring, the Peterson centralizer subalgebra of the nilHecke ring, and the FominStanley subalgebra of the nilCoxeter algebra. The discussion in Section 9 is new, and is largely motivated by a conjecture (Conjecture 5.6) of the author and Postnikov. In Section 10, we give a list of geometric interpretations and references for the objects studied in the earlier sections.

We have not intended to be comprehensive, especially with regards to generalizations and variations. There are four such which we must mention:

1. There is an important and well-developed connection between Stanley symmetric functions and Schubert polynomials, see [18, 108].

2. There is a theory of (affine) Stanley symmetric functions in classical types; see [19, $20,46,84,132]$.

3. Nearly all the constructions here have $K$-theoretic analogues. For full details see $[27,28,45,85]$.

4. There is a $t$-graded version of the theory. See $[92,95,96]$.

We have included exercises and problems throughout which occasionally assume more prerequisites. The exercises vary vastly in terms of difficulty. Some exercises essentially follow from the definitions, but other problems are questions for which the answers might not yet be known. 


\section{Stanley symmetric functions and reduced words}

For an integer $m \geq 1$, let $[m]=\{1,2, \ldots, m\}$. For a partition (or composition) $\lambda=$ $\left(\lambda_{1}, \lambda_{2}, \ldots, \lambda_{\ell}\right)$, we write $|\lambda|=\lambda_{1}+\cdots+\lambda_{\ell}$. The dominance order $\preceq$ on partitions is given by $\lambda \prec \mu$ if for some $k>0$ we have $\lambda_{1}+\lambda_{2}+\cdots+\lambda_{j}=\mu_{1}+\mu_{2}+\cdots+\mu_{j}$ for $1 \leq j<k$ and $\lambda_{1}+\lambda_{2}+\cdots+\lambda_{k}<\mu_{1}+\mu_{2}+\cdots+\mu_{k}$. The descent set $\operatorname{Des}(\mathbf{a})$ of a word $a_{1} a_{2} \cdots a_{n}$ is given by $\operatorname{Des}(\mathbf{a})=\left\{i \in[n-1] \mid a_{i}>a_{i+1}\right\}$.

\subsection{Young tableaux and Schur functions}

We shall assume the reader has some familiarity with symmetric functions and Young tableaux see Chapter 2, Section 1 or [120] [151, Ch. 7]. We write $\Lambda$ for the ring of symmetric functions. We let $m_{\lambda}$, where $\lambda$ is a partition, denote the monomial symmetric function, and let $h_{k}$ and $e_{k}$, for integers $k \geq 1$, denote the homogeneous and elementary symmetric functions respectively. For a partition $\lambda=\left(\lambda_{1}, \lambda_{2}, \ldots, \lambda_{\ell}\right)$, we define $h_{\lambda}:=h_{\lambda_{1}} h_{\lambda_{2}} \cdots h_{\lambda_{\ell}}$, and similarly for $e_{\lambda}$. We let $\langle.,$.$\rangle denote the Hall inner product of symmetric functions.$ Thus $\left\langle h_{\lambda}, m_{\mu}\right\rangle=\left\langle m_{\lambda}, h_{\mu}\right\rangle=\left\langle s_{\lambda}, s_{\mu}\right\rangle=\delta_{\lambda \mu}$.

We shall draw Young diagrams in French notation. A tableau of shape $\lambda$ is a filling of the Young diagram of $\lambda$ with integers. A tableau is column-strict (resp. row-strict) if it is increasing along columns (resp. rows). A tableau is standard if it is column-strict and row-strict, and uses each number $1,2, \ldots,|\lambda|$ exactly once. A tableau is semi-standard if it is column-strict, and weakly increasing along rows. Thus the tableaux

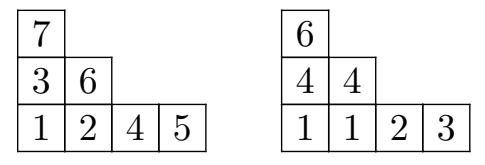

are standard and semistandard respectively. The weight $\operatorname{wt}(T)$ of a tableau $T$ is the composition $\left(\alpha_{1}, \alpha_{2}, \ldots\right)$ where $\alpha_{i}$ is equal to the number of $i$ 's in $T$. The Schur function $s_{\lambda}$ is given by

$$
s_{\lambda}\left(x_{1}, x_{2}, \ldots\right)=\sum_{T} x^{\mathrm{wt}(T)}
$$

where the summation is over semistandard tableaux of shape $\lambda$, and for a composition $\alpha$, we define $x^{\alpha}:=x_{1}^{\alpha_{1}} x_{2}^{\alpha_{2}} \ldots$. For a standard Young tableau $T$ we define $\operatorname{Des}(T)=$ $\{i \mid i+1$ is in a lower row than $i\}$. We also write $f^{\lambda}$ for the number of standard Young tableaux of shape $\lambda$. Similar definitions hold for skew shapes $\lambda / \mu$.

We shall often use the Jacobi-Trudi formula for Schur functions (see [120, 151]).

Theorem 1.1. Let $\lambda=\left(\lambda_{1} \geq \lambda_{2} \geq \cdots \geq \lambda_{\ell}>0\right)$ be a partition. Then

$$
s_{\lambda}=\operatorname{det}\left(h_{\lambda_{i}+j-i}\right)_{i, j=1}^{\ell} \text {. }
$$




\subsection{Permutations and reduced words}

Let $S_{n}$ denote the symmetric group of permutations on the letters $[n]$. We think of permutations $w, v \in S_{n}$ as bijections $[n] \rightarrow[n]$, so that the product $w v \in S_{n}$ is the composition $w \circ v$ as functions. The simple transposition $s_{i} \in S_{n}, i \in\{1,2, \ldots, n-1\}$ swaps the letters $i$ and $i+1$, keeping the other letters fixed. The symmetric group is generated by the $s_{i}$, with the relations

$$
\begin{aligned}
s_{i}^{2} & =1 & \text { for } 1 \leq i \leq n-1 \\
s_{i} s_{i+1} s_{i} & =s_{i+1} s_{i} s_{i+1} & \text { for } 1 \leq i \leq n-2 \\
s_{i} s_{j} & =s_{j} s_{i} & \text { for }|i-j|>1
\end{aligned}
$$

The length $\ell(w)$ of a permutation $w \in S_{n}$ is the length of the shortest expression $w=$ $s_{i_{1}} \cdots s_{i_{\ell}}$ for $w$ as a product of simple generators. Such a shortest expression is called a reduced expression for $w$, and the word $i_{1} i_{2} \cdots i_{\ell}$ is a reduced word for $w$. Let $R(w)$ denote the set of reduced words of $w \in S_{n}$. We usually write permutations in one-line notation, or alternatively give reduced words. For example $3421 \in S_{4}$ has reduced word 23123.

There is a natural embedding $S_{n} \hookrightarrow S_{n+1}$ and we will sometimes not distinguish between $w \in S_{n}$ and its image in $S_{n+1}$ under this embedding.

\subsection{Reduced words for the longest permutation}

The longest permutation $w_{0} \in S_{n}$ is $w_{0}=n(n-1) \cdots 21$ in one-line notation. Stanley [150] conjectured the following formula for the number of reduced words of $w_{0}$, which he then later proved using the theory of Stanley symmetric functions. Let $\delta_{n}=(n, n-1, \ldots, 1)$ denote the staircase of size $n$.

Theorem $1.2([150])$. The number $R\left(w_{0}\right)$ of reduced words for $w_{0}$ is equal to the number $f^{\delta_{n-1}}$ of staircase shaped standard Young tableaux.

\subsection{The Stanley symmetric function}

Definition 1.3 (Original definition). Let $w \in S_{n}$. Define the Stanley symmetric function ${ }^{3}$ $F_{w}$ by

$$
F_{w}\left(x_{1}, x_{2}, \ldots\right)=\sum_{\substack{1 \leq b_{1} \leq b_{2} \leq \cdots \leq b_{\ell} \\ a_{1} a_{2} \ldots a_{\ell} \in R(w) \\ a_{i}<a_{i+1}}} x_{b_{1}} x_{b_{2}} \cdots b_{b_{\ell}<b_{i+1}} .
$$

We shall establish the following fundamental result [150] in two different ways in Sections 2 and 5, but shall assume it for the remainder of this section.

Theorem 1.4 ([150]). The generating function $F_{w}$ is a symmetric function.

\footnotetext{
${ }^{3}$ Our conventions differ from Stanley's original definitions by $w \leftrightarrow w^{-1}$.
} 
A word $a_{1} a_{2} \cdots a_{\ell}$ is decreasing if $a_{1}>a_{2}>\cdots>a_{\ell}$. A permutation $w \in S_{n}$ is decreasing if it has a (necessarily unique) decreasing reduced word. The identity id $\in S_{n}$ is considered decreasing. A decreasing factorization of $w \in S_{n}$ is an expression $w=v_{1} v_{2} \cdots v_{r}$ such that $v_{i} \in S_{n}$ are decreasing, and $\ell(w)=\sum_{i=1}^{r} \ell\left(v_{i}\right)$.

Definition 1.5 (Decreasing factorizations). Let $w \in S_{n}$. Then

$$
F_{w}\left(x_{1}, x_{2}, \ldots\right)=\sum_{w=v_{1} v_{2} \cdots v_{r}} x_{1}^{\ell\left(v_{1}\right)} \cdots x_{r}^{\ell\left(v_{r}\right)} .
$$

Example 1.6. Consider $w=s_{1} s_{3} s_{2} s_{3} \in S_{4}$. Then $R(w)=\{1323,3123,1232\}$. Thus

$$
F_{w}=m_{211}+3 m_{1111}=s_{211} .
$$

The decreasing factorizations which give $m_{211}$ are $\overline{31} \overline{2} \overline{3}, \overline{1} \overline{32} \overline{3}, \overline{1} \overline{2} \overline{32}$.

\subsection{The code of a permutation}

Let $w \in S_{n}$. The code $c(w)$ is the sequence $c(w)=\left(c_{1}, c_{2}, \ldots\right)$ of nonnegative integers given by $c_{i}=\#\{j \in[n] \mid j>i$ and $w(j)<w(i)\}$ for $i \in[n]$, and $c_{i}=0$ for $i>n$. Note that the code of $w$ is the same regardless of which symmetric group it is considered an element of.

Let $\lambda(w)$ be the partition conjugate to the partition obtained from rearranging the parts of $c\left(w^{-1}\right)$ in decreasing order.

Example 1.7. Let $w=216534 \in S_{6}$. Then $c(w)=(1,0,3,2,0,0, \ldots)$, and $c\left(w^{-1}\right)=$ $(1,0,2,2,1,0, \ldots)$. Thus $\lambda(w)=(4,2)$.

For a symmetric function $f \in \Lambda$, let $\left[m_{\lambda}\right] f$ denote the coefficient of $m_{\lambda}$ in $f$.

Proposition 1.8 ([150]). Let $w \in S_{n}$.

1. Suppose $\left[m_{\lambda}\right] F_{w} \neq 0$. Then $\lambda \prec \lambda(w)$.

2. $\left[m_{\lambda(w)}\right] F_{w}=1$.

Proof. Left multiplication of $w$ by $s_{i}$ acts on $c\left(w^{-1}\right)$ by

$$
\left(c_{1}, \ldots, c_{i}, c_{i+1}, \ldots\right) \longmapsto\left(c_{1}, \ldots, c_{i+1}, c_{i}-1, \ldots\right)
$$

whenever $\ell\left(s_{i} w\right)<\ell(w)$. Thus factorizing a decreasing permutation $v$ out of $w$ from the left will decrease $\ell(v)$ different entries of $c\left(w^{-1}\right)$ each by 1 . (1) follows easily from this observation.

To obtain (2), one notes that there is a unique decreasing permutation $v$ of length $\mu_{1}(w)$ such that $\ell(w)=\ell\left(v^{-1} w\right)+\ell(v)$.

Example 1.9. Continuing Example 1.6, one has $w=2431$ in one-line notation. Thus $\lambda(w)=(2,1,1)$, agreeing with Proposition 1.8 and our previous calculation. 


\subsection{Fundamental Quasi-symmetric functions}

Let $D \subset[n-1]$. Define the (Gessel) fundamental quasi-symmetric function $L_{D}$ by

$$
L_{D}\left(x_{1}, x_{2}, \ldots\right)=\sum_{\substack{1 \leq b_{1} \leq b_{2} \cdots \leq b_{n} \\ i \in \bar{D} \stackrel{b_{i+1}>b_{i}}{\Longrightarrow}}} x_{b_{1}} x_{b_{2}} \cdots x_{b_{n}} .
$$

Note that $L_{D}$ depends not just on the set $D$ but also on $n$.

The descent set $\operatorname{Des}(T)$ of a standard Young tableau $T$ is the set of labels $i$, such that $i+1$ occurs in a higher row than $i$. A basic fact relating Schur functions and fundamental quasi-symmetric functions is:

Proposition 1.10. Let $\lambda$ be a partition. Then

$$
s_{\lambda}=\sum_{T} L_{\operatorname{Des}(T)}
$$

where the summation is over all standard Young tableaux of shape $\lambda$.

Definition 1.11 (Using quasi symmetric functions). Let $w \in S_{n}$. Then

$$
F_{w}\left(x_{1}, x_{2}, \ldots\right)=\sum_{\mathbf{a} \in R\left(w^{-1}\right)} L_{\operatorname{Des}(\mathbf{a})}
$$

Example 1.12. Continuing Example 1.6, we have $F_{w}=L_{2}+L_{1}+L_{3}$, where all subsets are considered subsets of [3]. Note that these are exactly the descent sets of the tableaux

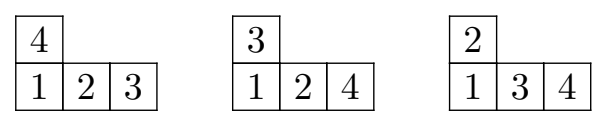

\subsection{Exercises}

1. Prove that $|c(w)|:=\sum_{i} c_{i}(w)$ is equal to $\ell(w)$.

2. Let $S_{\infty}=\cup_{n \geq 1} S_{n}$, where permutations are identified under the embeddings $S_{1} \hookrightarrow$ $S_{2} \hookrightarrow S_{3} \cdots$. Prove that $w \longmapsto c(w)$ is a bijection between $S_{\infty}$ and nonnegative integer sequences with finitely many non-zero entries.

3. Prove the equivalence of Definitions 1.3, 1.5, and 1.11.

4. What happens if we replace decreasing factorizations by increasing factorizations in Definition 1.5?

5. What is the relationship between $F_{w}$ and $F_{w^{-1}}$ ? 
6. (Grassmannian permutations) A permutation $w \in S_{n}$ is Grassmannian if it has at most one descent.

(a) Characterize the codes of Grassmannian permutations.

(b) Show that if $w$ is Grassmannian then $F_{w}$ is a Schur function.

(c) Which Schur functions are equal to $F_{w}$ for some Grassmannian permutation $w \in S_{n}$ ?

7. (321-avoiding permutations [18]) A permutation $w \in S_{n}$ is 321-avoiding if there does not exist $a<b<c$ such that $w(a)>w(b)>w(c)$. Show that $w$ is 321-avoiding if and only if no reduced word $\mathbf{i} \in R(w)$ contains a consecutive subsequence of the form $j(j+1) j$. If $w$ is 321-avoiding, show directly from the definition that $F_{w}$ is a skew Schur function.

\section{Edelman-Greene insertion}

\subsection{Insertion for reduced words}

We now describe an insertion algorithm for reduced words, due to Edelman and Greene [40], which establishes Theorem 1.4, and in addition stronger positivity properties. Related bijections were studied by Lascoux-Schützenberger [110] and by Haiman [62].

Let $T$ be a column and row strict Young tableau. The reading word $r(T)$ is the word obtained by reading the rows of $T$ from left to right, starting with the top row.

Let $w \in S_{n}$. We say that a tableau $T$ is a $E G$-tableau for $w$ if $r(T)$ is a reduced word for $w$. For example,

$$
T=\begin{array}{|l|l|l|}
\hline 2 & 3 & \\
\hline 1 & 2 & 3 \\
\hline
\end{array}
$$

has reading word $r(T)=23123$, and is an EG-tableau for $3421 \in S_{4}$.

Theorem 2.1 ([40]). Let $w \in S_{n}$. There is a bijection between $R(w)$ and the set of pairs $(P, Q)$, where $P$ is an EG-tableau for $w$, and $Q$ is a standard Young tableau with the same shape as $P$. Furthermore, under the bijection $\mathbf{i} \leftrightarrow(P(\mathbf{i}), Q(\mathbf{i}))$ we have $\operatorname{Des}(\mathbf{i})=\operatorname{Des}(Q)$.

Combining Theorem 2.1 with Proposition 1.10 and Definition 1.11, we obtain:

Corollary 2.2. Let $w \in S_{n}$. Then $F_{w}=\sum_{\lambda} \alpha_{w \lambda} s_{\lambda}$, where $\alpha_{w \lambda}$ is equal to the number of EG-tableau for $w^{-1}$. In particular, $F_{w}$ is Schur positive.

As a consequence we obtain Theorem 1.4.

Lemma 2.3. Suppose $T$ is an EG-tableau for $S_{n}$. Then the shape of $T$ is contained in the staircase $\delta_{n-1}$. 
Proof. Since $T$ is row-strict and column-strict, the entry in the $i$-th row and $j$-th column is greater than or equal to $i+j-1$. But EG-tableaux can only be filled with the numbers $1,2, \ldots, n-1$, so the shape of $T$ is contained inside $\delta_{n-1}$.

Proof of Theorem 1.2. The longest word $w_{0}$ has length $\left(\begin{array}{l}n \\ 2\end{array}\right)$. Suppose $T$ is an EG-tableau for $w_{0}$. Since the staircase $\delta_{n-1}$ has exactly $\left(\begin{array}{l}n \\ 2\end{array}\right)$ boxes, Lemma 2.3 shows that $T$ must have shape $\delta_{n-1}$. But then it is easy to see that the only possibility for $T$ is the tableau

\begin{tabular}{|c|c|c|c|c|}
\hline$n-1$ & & & & \\
\hline$\vdots$ & $\vdots$ & & & \\
\hline 3 & $\ldots$ & $n-1$ & & \\
\hline 2 & 3 & $\ldots$ & $n-1$ & \\
\hline 1 & 2 & 3 & $\ldots$ & $n-$ \\
\hline
\end{tabular}

Thus it follows from Theorem 2.1 that $R\left(w_{0}\right)=f^{\delta_{n-1}}$.

The proof of Theorem 2.1 is via an explicit insertion algorithm. Suppose $T$ is an EGtableau. We describe the insertion of a letter $a$ into $T$. If the largest letter in the first (that is, bottom) row of $T$ is less than $a$, then we add $a$ to the end of the first row, and the insertion is complete. Otherwise, we find the smallest letter $a^{\prime}$ in $T$ greater than $a$, and bump $a^{\prime}$ to the second row, where the insertion algorithm is recursively performed. The first row $R$ of $T$ changes as follows: if both $a$ and $a+1$ were present in $R$ (and thus $\left.a^{\prime}=a+1\right)$ then the row remains unchanged; otherwise, we replace $a^{\prime}$ by $a$ in $R$.

For a reduced word $\mathbf{i}=i_{1} i_{2} \cdots i_{\ell}$, we obtain $P(\mathbf{i})$ by inserting $i_{1}$, then $i_{2}$, and so on, into the empty tableau. The tableau $Q(\mathbf{i})$ is the standard Young tableau which records the changes in shape of the EG-tableau as this insertion is performed.

Example 2.4. Let $\mathbf{i}=21232$. Then the successive EG-tableau are

\begin{tabular}{|c|c|c|c|c|c|c|c|}
\hline 2 & \begin{tabular}{|l|}
2 \\
\end{tabular} & & \begin{tabular}{|l}
2 \\
\end{tabular} & & \begin{tabular}{|l|}
2 \\
\end{tabular} & 3 & \\
\hline 1 & & 2 & & \begin{tabular}{l|l}
2 & 3
\end{tabular} & 1 & 2 & 3 \\
\hline
\end{tabular}

so that

$$
Q(\mathbf{i})=\begin{array}{|l|l|l}
\hline 2 & 5 & \\
\hline 1 & 3 & 4 \\
\hline
\end{array}
$$




\subsection{Coxeter-Knuth relations}

Let $\mathbf{i}$ be a reduced word. A Coxeter-Knuth relation on $\mathbf{i}$ is one of the following transformations on three consecutive letters of $\mathbf{i}$ :

1. $a(a+1) a \sim(a+1) a(a+1)$

2. $a b c \sim a c b$ when $b<a<c$

3. $a b c \sim b a c$ when $b<c<a$

Since Coxeter-Knuth relations are in particular Coxeter relations for the symmetric group, it follows that if two words are related by Coxeter-Knuth relations then they represent the same permutation in $S_{n}$. The following result of Edelman and Greene states that Coxeter-Knuth equivalence is an analogue of Knuth-equivalence for reduced words.

Theorem $2.5([40])$. Suppose $\mathbf{i}, \mathbf{i}^{\prime} \in R(w)$. Then $P(\mathbf{i})=P\left(\mathbf{i}^{\prime}\right)$ if and only if $\mathbf{i}$ and $\mathbf{i}^{\prime}$ are Coxeter-Knuth equivalent.

\subsection{Exercises and Problems}

1. For $w \in S_{n}$ let $1 \times w \in S_{n+1}$ denote the permutation obtained from $w$ by adding 1 to every letter in the one-line notation, and putting a 1 in front. Thus if $w=24135$, we have $1 \times w=135246$. Show that $F_{w}=F_{1 \times w}$.

2. Suppose $w \in S_{n}$ is 321-avoiding (see Section 1.7). Show that Edelman-Greene insertion of $\mathbf{i} \in R(w)$ is the usual Robinson-Schensted insertion of $\mathbf{i}$.

3. (Vexillary permutations [18]) A permutation $w \in S_{n}$ is vexillary if it avoids the pattern 2143. That is, there do not exist $a<b<c<d$ such that $w(b)<w(a)<$ $w(d)<w(c)$. In particular, $w_{0}$ is vexillary.

The Stanley symmetric function $\tilde{F}_{w}$ is equal to a Schur function $s_{\lambda}$ if and only if $w$ is vexillary [18, p.367]. Is there a direct proof using Edelman-Greene insertion?

4. (Shape of a reduced word) The shape $\lambda(\mathbf{i})$ of a reduced word $\mathbf{i} \in R(w)$ is the shape of the tableau $P(\mathbf{i})$ or $Q(\mathbf{i})$ under Edelman-Greene insertion. Is there a direct way to read off the shape of a reduced word? (See [154] for a description of $\lambda_{1}(\mathbf{i})$.)

For example, Greene's invariants (see for example [151, Ch. 7]) describe the shape of a word under Robinson-Schensted insertion.

5. (Coxeter-Knuth relations and dual equivalence (graphs)) Show that Coxeter-Knuth relations on reduced words correspond exactly to elementary dual equivalences on the recording tableau (see [62]). They thus give a structure of a dual equivalence graph [5] on $R(w)$. 
An independent proof of this (in particular not using EG-insertion), together with the technology of [5], would give a new proof of the Schur positivity of Stanley symmetric functions.

6. (Lascoux-Schützenberger transition) Let $(i, j) \in S_{n}$ denote the transposition which swaps $i$ and $j$. Fix $r \in[n]$ and $w \in S_{n}$. The Stanley symmetric functions satisfy [110] the equality

$$
\sum_{u=w(r, s): \ell(u)=\ell(w)+1} F_{u}=\left(\sum_{v=s} F_{v}\right)\left(+F_{x}\right)
$$

where the last term with $x=(1 \times w)(1, r)$ is only present if $\ell(x)=\ell(w)+1$.

One obtains another proof of the Schur positivity of $F_{u}$ as follows. Let $r$ be the last descent of $u$, and let $k$ be the largest index such that $u(r)>u(k)$. Set $w=u(r, k)$. Then the left hand side of (2.1) has only one term $F_{u}$. Recursively repeating this procedure for the terms $F_{v}$ on the right hand side one obtains a positive expression for $F_{u}$ in terms of Schur functions.

7. (Little's bijection) Little [117] described an algorithm to establish (2.1), which we formulate in the manner of [86]. A v-marked nearly reduced word is a pair (i, $a$ ) where $\mathbf{i}=i_{1} i_{2} \cdots i_{\ell}$ is a word with letters in $\mathbb{Z}_{>0}$ and $a$ is an index such that $\mathbf{j}=$ $i_{1} i_{2} \cdots \hat{i}_{a} \cdots i_{\ell}$ is a reduced word for $v$, where $\hat{i}_{a}$ denotes omission. We say that $(\mathbf{i}, a)$ is a marked nearly reduced word if it is a $v$-marked nearly reduced word for some $v$. A marked nearly reduced word is a marked reduced word if $\mathbf{i}$ is reduced.

Define the directed Little graph on marked nearly reduced words, where each vertex has a unique outgoing edge $(\mathbf{i}, a) \rightarrow\left(\mathbf{i}^{\prime}, a^{\prime}\right)$ as follows: $\mathbf{i}^{\prime}$ is obtained from $\mathbf{i}$ by changing $i_{a}$ to $i_{a}-1$. If $i_{a}-1=0$, then we also increase every letter in $\mathbf{i}$ by 1 . If $\mathbf{i}$ is reduced then $a^{\prime}=a$. If $\mathbf{i}$ is not reduced then $a^{\prime}$ is the unique index not equal to $a$ such that $i_{1} i_{2} \cdots \hat{i}_{a^{\prime}} \cdots i_{\ell}$ is reduced. (Check that this is well-defined.)

For a marked reduced word ( $\mathbf{i}, a)$ such that $\mathbf{i}$ is reduced, the forward Little move sends $(\mathbf{i}, a)$ to $(\mathbf{j}, b)$ where $(\mathbf{j}, b)$ is the first marked reduced word encountered by traversing the Little graph.

Example 2.6. Beginning with $\mathbf{i}=2134323$ and $a=5$ one has

$$
2134321 \rightarrow 2134221 \rightarrow 2134211 \rightarrow 3245321 .
$$

Note that $\mathbf{i}$ is a reduced word for $u=53142$ which covers $w=43152$. The word 3245321 is a reduced word for $514263=(1 \times w)(1,2)$.

Check that if you apply the forward Little move to a $w$-marked reduced word $(\mathbf{i}, a)$ where $\mathbf{i} \in R(u)$ for some $u$ on the left hand side of (2.1), you will get a ( $w$ or $1 \times w)$ marked reduced word $(\mathbf{j}, b)$ where $\mathbf{j} \in R(v)$ for some $v$ on the right hand side of (2.1). This can then be used to prove (2.1). 
8. (Dual Edelman-Greene equivalence) Let $R(\infty)$ denote the set of all reduced words of permutations. We say that $\mathbf{i}, \mathbf{i}^{\prime} \in R(\infty)$ are dual EG-equivalent if the recording tableaux under EG-insertion are the same: $Q(\mathbf{i})=Q\left(\mathbf{i}^{\prime}\right)$.

Conjecture 2.7. Two reduced words are dual EG-equivalent if and only if they are connected by forward and backwards Little moves.

For example, both 2134321 and 3245321 of Example 2.6 Edelman-Greene insert to give recording tableau

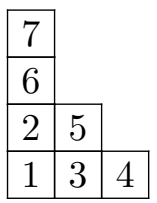

Hamaker and Young [63] have announced a proof of Conjecture 2.7.

9. Fix a symmetric group $S_{n}$. Is there a formula for the number of EG-tableau of a fixed shape $\lambda$ ? (See also Section 5.4 and compare with formulae for the number of (semi)standard tableaux [151].)

10. There are two common bijections which demonstrate the symmetry of Schur functions: the Bender-Knuth involution [10], and the Lascoux-Schützenberger/crystal operators (see for example [106]).

Combine this with Edelman-Greene insertion to obtain an explicit weight-changing bijection on the monomials of a Stanley symmetric function, which exhibits the symmetry of a Stanley symmetric function. Compare with Stanley's original bijection [150].

11. (Jeu-de-taquin for reduced words) There are Jeu-de-taquin theories for skew EGtableaux. This was developed for two-row tableaux by Bergeron and Sottile [15], and by Thomas and Yong $[153,154]$ in the context of Hecke insertion. For example one possible slide is

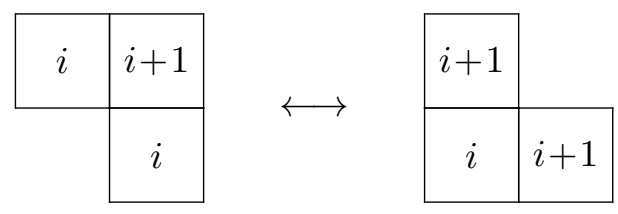

\section{Affine Stanley symmetric functions}

\subsection{Affine symmetric group}

For basic facts concerning the affine symmetric group, we refer the reader to Chapter 2, Section 1 and to [21]. 
Let $n>2$ be a positive integer. Let $\tilde{S}_{n}$ denote the affine symmetric group with simple generators $s_{0}, s_{1}, \ldots, s_{n-1}$ satisfying the relations

$$
\begin{aligned}
s_{i}^{2} & =1 & \text { for all } i \\
s_{i} s_{i+1} s_{i} & =s_{i+1} s_{i} s_{i+1} & \text { for all } i \\
s_{i} s_{j} & =s_{j} s_{i} & \text { for }|i-j| \geq 2 .
\end{aligned}
$$

Here and elsewhere, the indices will be taken modulo $n$ without further mention. The length $\ell(w)$ and reduced words $R(w)$ for affine permutations $w \in \tilde{S}_{n}$ are defined in an analogous manner to Section 1.2. The symmetric group $S_{n}$ embeds in $\tilde{S}_{n}$ as the subgroup generated by $s_{1}, s_{2}, \ldots, s_{n-1}$.

One may realize $\tilde{S}_{n}$ as the set of all bijections $w: \mathbb{Z} \rightarrow \mathbb{Z}$ such that $w(i+n)=w(i)+n$ for all $i$ and $\sum_{i=1}^{n} w(i)=\sum_{i=1}^{n} i$. In this realization, to specify an element $w \in \widetilde{S}_{n}$ it suffices to give the "window" $[w(1), w(2), \ldots, w(n)]$. The product $w \cdot v$ of two affine permutations is then the composed bijection $w \circ v: \mathbb{Z} \rightarrow \mathbb{Z}$. Thus $w s_{i}$ is obtained from $w$ by swapping the values of $w(i+k n)$ and $w(i+k n+1)$ for every $k \in \mathbb{Z}$. An affine permutation $w \in \tilde{S}_{n}$ is Grassmannian if $w(1)<w(2)<\cdots<w(n)$. For example, the affine Grassmannian permutation $[-2,2,6] \in \tilde{S}_{3}$ has reduced words 2120 and 1210 . We denote by $\tilde{S}_{n}^{0} \subset \tilde{S}_{n}$ the subset of affine Grassmannian permutations.

\subsection{Definition}

A word $a_{1} a_{2} \cdots a_{\ell}$ with letters in $\mathbb{Z} / n \mathbb{Z}$ is called cyclically decreasing if (1) each letter occurs at most once, and (2) whenever $i$ and $i+1$ both occur in the word, $i+1$ precedes $i$.

An affine permutation $w \in \tilde{S}_{n}$ is called cyclically decreasing if it has a cyclically decreasing reduced word. Note that such a reduced word may not be unique.

Lemma 3.1. There is a bijection between strict subsets of $\mathbb{Z} / n \mathbb{Z}$ and cyclically decreasing affine permutations $w \in \tilde{S}_{n}$, sending a subset $S$ to the unique cyclically decreasing affine permutation which has reduced word using exactly the simple generators $\left\{s_{i} \mid i \in S\right\}$.

We define cyclically decreasing factorizations of $w \in \tilde{S}_{n}$ in the same way as decreasing factorizations in $S_{n}$. See also Chapter 2, Section 1.3 where cyclically decreasing factorizations are discussed from the point of view of $k$-bounded partitions and $(k+1)$-cores.

Definition 3.2. Let $w \in \tilde{S}_{n}$. The affine Stanley symmetric function $\tilde{F}_{w}$ is given by

$$
\tilde{F}_{w}=\sum_{w=v_{1} v_{2} \cdots v_{r}} x_{1}^{\ell\left(v_{1}\right)} x_{2}^{\ell\left(v_{2}\right)} \cdots x_{r}^{\ell\left(v_{r}\right)}
$$

where the summation is over cyclically decreasing factorizations of $w$.

Theorem 3.3 ([78]). The generating function $\tilde{F}_{w}$ is a symmetric function. 
Theorem 3.3 can be proved directly, as was done in [78]. We shall establish Theorem 3.3 using the technology of the affine nilHecke algebra in Sections 6-8. Some immediate observations:

1. $\tilde{F}_{w}$ is a homogeneous of degree $\ell(w)$.

2. If $w \in S_{n}$, then a cyclically decreasing factorization of $w$ is just a decreasing factorization of $w$, so $\tilde{F}_{w}=F_{w}$.

3. The coefficient of $x_{1} x_{2} \cdots x_{\ell(w)}$ in $\tilde{F}_{w}$ is equal to $|R(w)|$.

Example 3.4. Consider the affine permutation $w=s_{2} s_{1} s_{2} s_{0} s_{2}$. The reduced words are $R(w)=\{21202,12102,21020\}$. The other cyclically decreasing factorizations are

$\overline{21} \overline{2} \overline{0} \overline{2}, \overline{2} \overline{1} \overline{2} \overline{02}, \overline{1} \overline{21} \overline{0} \overline{2}, \overline{1} \overline{2} \overline{1} \overline{02}, \overline{1} \overline{2} \overline{10} \overline{2}, \overline{21} \overline{0} \overline{2} \overline{0}, \overline{2} \overline{1} \overline{02} \overline{0}, \overline{2} \overline{10} \overline{2} \overline{0}$

$\overline{21} \overline{2} \overline{02}, \overline{1} \overline{21} \overline{02}, \overline{21} \overline{02} \overline{0}$

Thus

$$
\tilde{F}_{w}=m_{221}+2 m_{2111}+3 m_{11111} .
$$

\subsection{Codes}

Let $w \in \tilde{S}_{n}$. The code $c(w)$ is a vector $c(w)=\left(c_{1}, c_{2}, \ldots, c_{n}\right) \in \mathbb{Z}_{\geq 0}^{n}-\mathbb{Z}_{>0}^{n}$ of non-negative entries with at least one 0 . The entries are given by $c_{i}=\#\{j \in \mathbb{Z} \mid j>i$ and $w(j)<w(i)\}$.

It is shown in [21] that there is a bijection between codes and affine permutations and that $\ell(w)=|c(w)|:=\sum_{i=1}^{n} c_{i}$. We define $\lambda(w)$ as for usual permutations (see Section 1). For example, for $w=s_{2} s_{0} s_{1} s_{2} s_{1} s_{0}=[-4,3,7] \in \tilde{S}_{3}$, one has $c\left(w^{-1}\right)=(5,1,0)$ and $\lambda=(2,1,1,1,1)$.

Let $\mathcal{B}^{n}$ denote the set of partitions $\lambda$ satisfying $\lambda_{1}<n$, called the set of $(n-1)$-bounded partitions.

Lemma $3.5([21])$. The map $w \mapsto \lambda(w)$ is a bijection between $\tilde{S}_{n}^{0}$ and $\mathcal{B}^{n}$.

The analogue of Proposition 1.8 has a similar proof.

Proposition 3.6 ([78]). Let $w \in \tilde{S}_{n}$.

1. Suppose $\left[m_{\lambda}\right] F_{w} \neq 0$. Then $\lambda \prec \lambda(w)$.

2. $\left[m_{\lambda(w)}\right] F_{w}=1$. 


\section{$3.4 \Lambda_{(n)}$ and $\Lambda^{(n)}$}

Let $\Lambda_{(n)} \subset \Lambda$ be the subalgebra generated by $h_{1}, h_{2}, \ldots, h_{n-1}$, and let $\Lambda^{(n)}:=\Lambda / I_{(n)}$ where $I_{(n)}$ is the ideal generated by $m_{\mu}$ for $\mu \notin \mathcal{B}^{n}$. A basis for $\Lambda_{(n)}$ is given by $\left\{h_{\lambda} \mid \lambda \in \mathcal{B}^{n}\right\}$. A basis for $\Lambda^{(n)}$ is given by $\left\{m_{\lambda} \mid \lambda \in \mathcal{B}^{n}\right\}$. Note that the rings $\Lambda_{(n)}$ and $\Lambda^{(n)}$ are denoted $\Lambda_{(k)}$ and $\Lambda^{(k)}$ in Chapter 2, where $k=n-1$.

The ring of symmetric functions $\Lambda$ is a Hopf algebra, with coproduct given by $\Delta\left(h_{k}\right)=$ $\sum_{j=0}^{k} h_{j} \otimes h_{k-j}$. Equivalently, the coproduct of $f\left(x_{1}, x_{2}, \ldots\right) \in \Lambda$ can be obtained by writing $f\left(x_{1}, x_{2}, \ldots, y_{1}, y_{2}, \ldots\right)$ in the form $\sum_{i} f_{i}\left(x_{1}, x_{2}, \ldots\right) \otimes g_{i}\left(y_{1}, y_{2}, \ldots\right)$ where $f_{i}$ and $g_{i}$ are symmetric in $x$ 's and $y$ 's respectively. Then $\Delta(f)=\sum_{i} f_{i} \otimes g_{i}$.

The ring $\Lambda$ is self Hopf-dual under the Hall inner product. That is, one has $\langle\Delta f, g \otimes h\rangle=$ $\langle f, g h\rangle$ for $f, g, h \in \Lambda$. Here the Hall inner product is extended to $\Lambda \otimes \Lambda$ in the obvious way. The rings $\Lambda_{(n)}$ and $\Lambda^{(n)}$ are in fact Hopf algebras, which are dual to each other under the same inner product. We refer the reader to [120] for further details.

\subsection{Affine Schur functions}

Stanley symmetric functions expand positively in terms of the basis of Schur functions (Corollary 2.2). We now describe the analogue of Schur functions for the affine setting.

For $\lambda \in \mathcal{B}^{n}$, we let $\tilde{F}_{\lambda}:=\tilde{F}_{w}$ where $w \in \tilde{S}_{n}^{0}$ is the unique affine Grassmannian permutation with $\lambda(w)=\lambda$. These functions $\tilde{F}_{\lambda}$ are called affine Schur functions (or dual $k$-Schur functions, or weak Schur functions).

Theorem $3.7([96,78])$. The affine Schur functions $\left\{\tilde{F}_{\lambda} \mid \lambda \in \mathcal{B}^{n}\right\}$ form a basis of $\Lambda^{(n)}$.

Proof. By Proposition 3.6, the leading monomial term of $\tilde{F}_{\lambda}$ is $m_{\lambda}$. Thus $\left\{\tilde{F}_{\lambda} \mid \lambda \in \mathcal{B}^{n}\right\}$ is triangular with respect to the basis $\left\{m_{\lambda} \mid \lambda \in \mathcal{B}^{n}\right\}$, so that it is also a basis.

We let $\left\{s_{\lambda}^{(k)}\right\} \subset \Lambda_{(n)}$ denote the dual basis to $\tilde{F}_{\lambda}$. These are the (ungraded) $k$-Schur functions, where $k=n-1$. It turns out that the $k$-Schur functions are Schur positive. However, affine Stanley symmetric functions are not. Instead, one has:

Theorem $3.8([79])$. The affine Stanley symmetric functions $\tilde{F}_{w}$ expand positively in terms of the affine Schur functions $\tilde{F}_{\lambda}$.

Theorem 3.8 was established using geometric methods. See Section 10 and [79]. It is an open problem to give a combinatorial interpretation of the affine Stanley coefficients, expressing affine Stanley symmetric functions in terms of affine Schur functions.

\subsection{Example: The case of $\tilde{S}_{3}$}

To illustrate Theorem 3.7, we completely describe the affine Schur functions for $\tilde{S}_{3}$. 
Proposition 3.9. Let $w \in \tilde{S}_{n}$ be the affine Grassmannian permutation corresponding to the partition $\left(2^{a} 1^{b}\right)$. Then $|R(w)|=\left(\begin{array}{c}\lfloor b / 2+a\rfloor \\ a\end{array}\right)$.

Proposition 3.10. The affine Schur function $\tilde{F}_{2^{a}, 1^{b}}$ is given by

$$
\tilde{F}_{2^{a}, 1^{b}}=\sum_{j=0}^{a}\left(\begin{array}{c}
\lfloor b / 2+a-j\rfloor \\
a-j
\end{array}\right) m_{2^{j} 1^{b+2 a-2 j}} .
$$

The $k$-Schur function $s_{2^{a}, 1^{b}}^{(2)}$ is given by

$$
s_{2^{a}, 1^{b}}^{(2)}=h_{2}^{a} e_{2}^{\lfloor b / 2\rfloor} h_{1}^{b-2\lfloor b / 2\rfloor} .
$$

Example 3.11. For $w=1210$, we have $a=1$ and $b=2$. Thus $R(w)=\{1210,2120\}$ has cardinality $\left(\begin{array}{l}2 \\ 1\end{array}\right)=2$, and $\tilde{F}_{2,1^{2}}=m_{211}+2 m_{1111}$.

Example 3.12. The affine Stanley symmetric function of Example 3.4 expands as

$$
\tilde{F}_{w}=\tilde{F}_{2^{2}, 1}+\tilde{F}_{2,1^{3}}+\tilde{F}_{1^{5}}
$$

agreeing with Theorem 3.8 .

\subsection{Exercises and problems}

1. (Coproduct formula [78]) Show that $\Delta \tilde{F}_{w}=\sum_{u v=w: \ell(w)=\ell(u)+\ell(v)} \tilde{F}_{u} \otimes \tilde{F}_{v}$.

2. (321-avoiding affine permutations [78]) Extend the results in Section 1.7 on 321avoiding permutations to the affine case.

3. (Affine vexillary permutations) For which $w \in \tilde{S}_{n}$ is $\tilde{F}_{w}$ equal to an affine Schur function $\tilde{F}_{\lambda}$ ? See the discussion of vexillary permutations in Section 2.3 and also [78, Problem 1].

4. ( $n \rightarrow \infty$ limit) Show that for a fixed partition $\lambda$, we have $\lim _{n \rightarrow \infty} \tilde{F}_{\lambda}^{(n)}=s_{\lambda}$, where $\tilde{F}^{(n)}$ denotes the affine Schur function for $\tilde{S}_{n}$.

5. Extend Proposition 3.10 to all affine Stanley symmetric functions in $\tilde{S}_{3}$, and thus give a formula for the affine Stanley coefficients.

6. Is there an affine analogue of the fundamental quasi-symmetric functions? For example, one might ask that affine Stanley symmetric functions expand positively in terms of such a family of quasi-symmetric functions. Affine Stanley symmetric functions do not in general expand positively in terms of fundamental quasi-symmetric functions (see [121, Theorem 5.7]). 
7. Find closed formulae for numbers of reduced words in the affine symmetric groups $\tilde{S}_{n}$, $n>3$, extending Proposition 3.9. Are there formulae similar to the determinantal formula, or hook-length formula for the number of standard Young tableaux?

8. ( $n$-cores) A skew shape $\lambda / \mu$ is a $n$-ribbon if it is connected, contains $n$ squares, and does not contain a $2 \times 2$ square. An $n$-core $\lambda$ is a partition such that there does not exist $\mu$ so that $\lambda / \mu$ is a $n$-ribbon. There is a bijection between the set of $n$-cores and the affine Grassmannian permutaitons $\tilde{S}_{n}^{0}$. Affine Schur functions can be described in terms of tableaux on $n$-cores, called $k$-tableau [95] (or weak tableau in [81]). See Chapter 2 for further details.

9. (Cylindric Schur functions $[133,121])$ Let $C(k, n)$ denote the set of lattice paths $p$ in $\mathbb{Z}^{2}$ where every step either goes up or to the right, and which is invariant under the translation $(x, y) \mapsto(x+n-k, y+k)$. Such lattice paths can be thought of as the boundary of an infinite periodic Young diagram, or equivalently of a Young diagram on a cylinder. We write $p \subset q$ if $p$ lies completely to the left of $q$. A cylindric skew semistandard tableau is a sequence $p_{0} \subset p_{1} \subset \cdots \subset p_{k}$ of $p_{i} \in C(k, n)$ where the region between $p_{i}$ and $p_{i+1}$ does not contain two squares in the same column. One obtains [133] a natural notion of a cylindric (skew) Schur function. Show that every cylindric Schur function is an affine Stanley symmetric function, and every affine Stanley symmetric function of a 321-avoiding permutation is a cylindric Schur function $([78])$.

10. (Kashiwara-Shimozono affine Grothendieck polynomials) The usual Stanley symmetric functions can be expressed as stable limits of Schubert polynomials [18]. What is the relationship between affine Stanley symmetric functions and the affine Grothendieck polynomials of Kashiwara and Shimozono [69]?

11. Is there a good notion of Coxeter-Knuth equivalence for reduced words of affine permutations? This may have an application to the affine Schur positivity of affine Stanley symmetric functions (Theorem 3.8). See also Section 2.3 (5).

12. (Affine Little bijection [86]) There is an affine analogue of Little's bijection (Section 2.3) developed in [86]. It gives a combinatorial proof of the affine analogue of the transition formula (2.1). Can the affine Little bijection, or the affine transition formula lead to a proof of Theorem 3.8? Can one define a notion of dual EG-equivalence using the affine Little bijection?

13. (Branching positivity $[82,80])$ Let $\tilde{F}_{\lambda}^{(n)}$ denote the affine Schur functions for $\tilde{S}_{n}$. Then $\tilde{F}_{\lambda}^{(n+1)}$ expands positively in terms of $\tilde{F}_{\mu}^{(n)}$ modulo the ideal in symmetric functions generated by $m_{\nu}$ with $\nu_{1} \geq n$. Deduce using (4) that $k$-Schur functions are Schur positive. 


\section{Root systems and Weyl groups}

In this section, we let $W$ be a finite Weyl group and $W_{\text {af }}$ denote the corresponding affine Weyl group. We shall assume basic familiarity with Weyl groups, root systems, and weights $[64,67]$.

\subsection{Notation for root systems and Weyl groups}

Let $A=\left(a_{i j}\right)_{i, j \in I_{\text {af }}}$ denote an affine Cartan matrix, where $I_{\text {af }}=I \cup\{0\}$, so that $\left(a_{i j}\right)_{i, j \in I}$ is the corresponding finite Cartan matrix. For example, for type $\tilde{A}_{n-1}$ (corresponding to $\left.\tilde{S}_{n}\right)$ and $n>2$ we have $I_{\text {af }}=\mathbb{Z} / n \mathbb{Z}$ and

$$
a_{i j}= \begin{cases}2 & \text { if } i=j \\ -1 & \text { if } j=i \pm 1 \\ 0 & \text { otherwise. }\end{cases}
$$

The affine Weyl group $W_{\text {af }}$ is generated by involutions $\left\{s_{i} \mid i \in I_{\text {af }}\right\}$ satisfying the relations $\left(s_{i} s_{j}\right)^{m_{i j}}=\mathrm{id}$, where for $i \neq j$, one defines $m_{i j}$ to be $2,3,4,6, \infty$ according as $a_{i j} a_{j i}$ is $0,1,2,3, \geq 4$. The finite Weyl group $W$ is generated by $\left\{s_{i} \mid i \in I\right\}$. For the symmetric group $W=S_{n}$, we have $I=[n-1], m_{i, i+1}=3$, and $m_{i j}=2$ for $|i-j| \geq 2$.

Let $R$ be the root system for $W$. Let $R^{+}$denote the positive roots, $R^{-}$denote the negative roots and $\left\{\alpha_{i} \mid i \in I\right\}$ denote the simple roots. Let $\theta$ denote the highest root of $R^{+}$. Let $\rho=\frac{1}{2} \sum_{\alpha \in R^{+}} \alpha$ denote the half sum of positive roots. Also let $\left\{\alpha_{i}^{\vee} \mid i \in I\right\}$ denote the simple coroots.

We write $R_{\mathrm{af}}$ and $R_{\mathrm{af}}^{+}$for the affine root system, and positive affine roots. The positive simple affine roots (resp. coroots) are $\left\{\alpha_{i} \mid i \in I_{\text {af }}\right\}$ (resp. $\left\{\alpha_{i}^{\vee} \mid i \in I_{\text {af }}\right\}$ ). The null root $\delta$

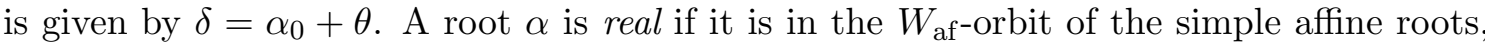
and imaginary otherwise. The imaginary roots are exactly $\{k \delta \mid k \in \mathbb{Z} \backslash\{0\}\}$. Every real affine root is of the form $\alpha+k \delta$, where $\alpha \in R$. The root $\alpha+k \delta$ is positive if $k>0$, or if $k=0$ and $\alpha \in R^{+}$.

Let $Q=\oplus_{i \in I} \mathbb{Z} \cdot \alpha_{i}$ denote the root lattice and let $Q^{\vee}=\oplus_{i \in I} \mathbb{Z} \cdot \alpha_{i}^{\vee}$ denote the co-root lattice. Let $P$ and $P^{\vee}$ denote the weight lattice and co-weight lattice respectively. Thus $Q \subset P$ and $Q^{\vee} \subset P^{\vee}$. We also have a map $Q_{\mathrm{af}}=\oplus_{i \in I_{\mathrm{af}}} \mathbb{Z} \cdot \alpha_{i} \rightarrow P$ given by sending $\alpha_{0}$ to $-\theta$ (or equivalently, by sending $\delta$ to 0 ). Let $\langle.,$.$\rangle denote the pairing between P$ and $P^{\vee}$. In particular, one requires that $\left\langle\alpha_{i}^{\vee}, \alpha_{j}\right\rangle=a_{i j}$.

The Weyl group acts on weights via $s_{i} \cdot \lambda=\lambda-\left\langle\alpha_{i}^{\vee}, \lambda\right\rangle \alpha_{i}$ (and via the same formula on $Q$ or $Q_{\mathrm{af}}$ ), and on coweights via $s_{i} \cdot \mu=\mu-\left\langle\mu, \alpha_{i}\right\rangle \alpha_{i}^{\vee}$ (and via the same formula on $\left.Q^{\vee}\right)$. For a real root $\alpha$ (resp. coroot $\left.\alpha^{\vee}\right)$, we let $s_{\alpha}$ (resp. $\left.s_{\alpha^{\vee}}\right)$ denote the corresponding reflection, defined by $s_{\alpha}=w r_{i} w^{-1}$ if $\alpha=w \cdot \alpha_{i}$. The reflection $s_{\alpha}$ acts on weights by $s_{\alpha} \cdot \lambda=\lambda-\left\langle\alpha^{\vee}, \lambda\right\rangle \alpha$. 
Example 4.1. Suppose $W=S_{n}$ and $W_{\text {af }}=\tilde{S}_{n}$. We have positive simple roots $\alpha_{1}, \alpha_{2}, \ldots, \alpha_{n-1}$ and an affine simple root $\alpha_{0}$. The finite positive roots are $R^{+}=\left\{\alpha_{i, j}:=\alpha_{i}+\alpha_{i+1} \cdots+\alpha_{j-1} \mid\right.$ $1 \leq i<j \leq n\}$. The reflection $s_{\alpha_{i, j}}$ is the transposition $(i, j)$. The highest root is $\theta=\alpha_{1}+\cdots+\alpha_{n-1}$. The affine positive roots are $R_{\mathrm{af}}^{+}=\left\{\alpha_{i, j} \mid i<j\right\}$, where for simple roots the index is taken modulo $n$. Note that one has $\alpha_{i, j}=\alpha_{i+n, j+n}$. The imaginary roots are of the form $\alpha_{i, i+k n}$. For a real root $\alpha_{i, j}$, the reflection $s_{\alpha_{i, j}}$ is the affine transposition $(i, j)$.

The weight lattice can be taken to be $P=\mathbb{Z}^{n} /(1,1, \ldots, 1)$, and the coweight lattice to be $P^{\vee}=\left\{\left(x_{1}, x_{2}, \ldots, x_{n}\right) \in \mathbb{Z}^{n} \mid \sum_{i} x_{i}=0\right\}$. The roots and coroots are then $\alpha_{i, j}=$ $e_{i}-e_{j}=\alpha_{i, j}^{\vee}$ (though the former is in $P$ and the latter is in $P^{\vee}$ ). The inner product $P^{\vee} \times P \rightarrow \mathbb{Z}$ is induced by the obvious one on $\mathbb{Z}^{n}$.

\subsection{Affine Weyl group and translations}

The affine Weyl group can be expressed as the semi-direct product $W_{\text {af }}=W \ltimes Q^{\vee}$, as follows. For each $\lambda \in Q^{\vee}$, one has a translation element $t_{\lambda} \in W_{\text {af }}$. Translations are multiplicative, so that $t_{\lambda} \cdot t_{\mu}=t_{\lambda+\mu}$. We also have the conjugation formula $w t_{\lambda} w^{-1}=t_{w \cdot \lambda}$ for $w \in W$ and $\lambda \in Q^{\vee}$. Let $s_{0}$ denote the additional simple generator of $W_{\mathrm{af}}$. Then translation elements are related to the simple generators via the formula

$$
s_{0}=s_{\theta} \vee t_{-\theta^{\vee}} .
$$

Example 4.2. For $W_{\text {af }}=\tilde{S}_{n}$, and $\lambda=\left(\lambda_{1}, \lambda_{2}, \ldots, \lambda_{n}\right) \in Q^{\vee}$, we have

$$
t_{\lambda}=\left[1+n \lambda_{1}, 2+n \lambda_{2}, \ldots, n+n \lambda_{n}\right] .
$$

Thus $t_{-\theta^{\vee}}=[1-n, 2, \ldots, n-1,2 n]$ and $s_{0}=s_{\theta} \vee t_{-\theta^{\vee}}$ is the equality

$$
[0,2, \ldots, n-1, n+1]=[n, 2, \ldots, n-1,1] \cdot[1-n, 2, \ldots, n-1,2 n] .
$$

The element $w t_{\lambda}$ acts on $\mu \in P$ via

$$
w t_{\lambda} \cdot \mu=w \cdot \mu \text {. }
$$

In other words, the translations act trivially on the finite weight lattice. This action is called the level zero action.

Let $\ell: W_{\mathrm{af}} \rightarrow \mathbb{Z}_{\geq 0}$ denote the length function of $W_{\mathrm{af}}$. Thus $\ell(w)$ is the length of the shortest reduced factorization of $w$.

Exercise 4.3. For $w t_{\lambda} \in W_{\mathrm{af}}$, we have

$$
\ell\left(w t_{\lambda}\right)=\sum_{\alpha \in R^{+}}|\langle\lambda, \alpha\rangle+\chi(w \cdot \alpha)|,
$$

where $\chi(\alpha)=0$ if $\alpha \in R^{+}$and $\chi(\alpha)=1$ otherwise. 
A coweight $\lambda$ is dominant (resp. anti-dominant) if $\langle\lambda, \alpha\rangle \geq 0$ (resp. $\leq 0$ ) for every root $\alpha \in R^{+}$.

Exercise 4.4. Suppose $\lambda \in Q^{\vee}$ is dominant. Then $\ell\left(t_{w \lambda}\right)=2\langle\lambda, \rho\rangle$.

Let $W_{\mathrm{af}}^{0}$ denote the minimal length coset representatives of $W_{\mathrm{af}} / W$, which we call Grassmannian elements. There is a natural bijection between $W_{\mathrm{af}}^{0}$ and $Q^{\vee}$ : each coset $W_{\mathrm{af}} / W$ contains one element from each set.

Exercise 4.5. We have $W_{\text {af }}^{0} \cap Q^{\vee}=Q^{-}$, the elements of the coroot lattice which are anti-dominant.

In fact an element $w t_{\lambda}$ lies in $W_{\text {af }}^{0}$ if and only if $t_{\lambda} \in Q^{-}$and $w \in W^{\lambda}$ where $W^{\lambda}$ is the set of minimal length representatives of $W / W_{\lambda}$ and $W_{\lambda}$ is the stabilizer subgroup of $\lambda$.

\section{NilCoxeter algebra and Fomin-Stanley construction}

Let $W$ be a Weyl group and $W_{\text {af }}$ be the corresponding affine Weyl group.

\subsection{The nilCoxeter algebra}

The nilCoxeter algebra $\mathbb{A}_{0}$ is the algebra over $\mathbb{Z}$ generated by $\left\{A_{i} \mid i \in I\right\}$ with relations

$$
\begin{array}{rlrl}
A_{i}^{2} & =0 & \\
\left(A_{i} A_{j}\right)^{b} & =\left(A_{j} A_{i}\right)^{b} & & \\
A_{j}\left(A_{i} A_{j}\right)^{b} & =\left(A_{i} A_{j}\right)^{b} A_{i} & \text { if }\left(s_{i} s_{j}\right)^{b}=\left(s_{j} s_{i}\right)^{b} & \text { if } s_{j}\left(s_{i} s_{j}\right)^{b}=\left(s_{i} s_{j}\right)^{b} s_{i}
\end{array}
$$

The algebra $\mathbb{A}_{0}$ is graded, where $A_{i}$ is given degree 1.

The corresponding algebra for the affine Weyl group will be denoted $\left(\mathbb{A}_{a f}\right)_{0}$. This algebra is also discussed in Chapter 2, Section 2.5.

Proposition 5.1. The nilCoxeter algebra $\mathbb{A}_{0}$ has basis $\left\{A_{w} \mid w \in W\right\}$, where $A_{w}=$ $A_{i_{1}} A_{i_{2}} \cdots A_{i_{\ell}}$ for any reduced word $i_{1} i_{2} \cdots i_{\ell}$ of $w$. The mulitplication is given by

$$
A_{w} A_{v}= \begin{cases}A_{w v} & \text { if } \ell(w v)=\ell(w)+\ell(v) \\ 0 & \text { otherwise. }\end{cases}
$$

\subsection{Fomin and Stanley's construction}

Suppose $W=S_{n}$. We describe the construction of Stanley symmetric functions of Fomin and Stanley [48]. Define

$$
\mathbf{h}_{k}=\sum_{w \text { decreasing: } \ell(w)=k} A_{w} .
$$


For example, when $n=4$, we have

$$
\begin{aligned}
& \mathbf{h}_{0}=\mathrm{id} \\
& \mathbf{h}_{1}=A_{1}+A_{2}+A_{3} \\
& \mathbf{h}_{2}=A_{21}+A_{31}+A_{32} \\
& \mathbf{h}_{3}=A_{321}
\end{aligned}
$$

where $A_{i_{1} i_{2} \cdots i_{\ell}}:=A_{i_{1}} A_{i_{2}} \cdots A_{i_{\ell}}$.

Lemma $5.2([48])$. The generating function $\mathbf{h}(t)=\sum_{k} \mathbf{h}_{k} t^{k}$ has the product expansion

$$
\mathbf{h}(t)=\left(1+t A_{n-1}\right)\left(1+t A_{n-2}\right) \cdots\left(1+t A_{1}\right) .
$$

Definition 5.3 (NilCoxeter algebra). The Stanley symmetric function $F_{w}$ is the coefficient of $A_{w}$ in the product

$$
\mathbf{h}\left(x_{1}\right) \mathbf{h}\left(x_{2}\right) \cdots
$$

Lemma 5.4 ([48]). We have

$$
\mathbf{h}(x) \mathbf{h}(y)=\mathbf{h}(y) \mathbf{h}(x)
$$

Thus for every $k, l$ we have

$$
\mathbf{h}_{k} \mathbf{h}_{l}=\mathbf{h}_{l} \mathbf{h}_{k} .
$$

Proof. We observe that $\left(1+x A_{i}\right)$ and $\left(1+y A_{j}\right)$ commute whenever $|i-j| \geq 2$ and that

$$
\left(1+x A_{i+1}\right)\left(1+x A_{i}\right)\left(1+y A_{i+1}\right)=\left(1+y A_{i+1}\right)\left(1+y A_{i}\right)\left(1+x A_{i+1}\right)\left(1-y A_{i}\right)\left(1+x A_{i}\right) .
$$

Assuming by induction that the result is true for $S_{n-1}$ we calculate

$$
\begin{aligned}
& \left(1+x A_{n-1}\right) \cdots\left(1+x A_{1}\right)\left(1+y A_{n-1}\right) \cdots\left(1+y A_{1}\right) \\
& =\left[\left(1+y A_{n-1}\right)\left(1+y A_{n-2}\right)\left(1+x A_{n-1}\right)\left(1-y A_{n-2}\right)\left(1+x A_{n-2}\right)\right] \\
& \left(1+x A_{n-3}\right) \cdots\left(1+x A_{1}\right)\left(1+y A_{n-2}\right) \cdots\left(1+y A_{1}\right) \\
& =\left(1+y A_{n-1}\right)\left(1+y A_{n-2}\right)\left(1+x A_{n-1}\right)\left(1-y A_{n-2}\right)\left[\left(1+y A_{n-2}\right) \cdots\left(1+y A_{1}\right)\right] \\
& {\left[\left(1+x A_{n-2}\right) \cdots\left(1+x A_{1}\right)\right]} \\
& =\left(1+y A_{n-1}\right) \cdots\left(1+y A_{1}\right)\left(1+x A_{n-1}\right) \cdots\left(1+x A_{1}\right) .
\end{aligned}
$$

Proof of Theorem 1.4. Follows immediately from Definition 5.3 and Lemma 5.4.

The following corollary of Lemma 5.4 suggests a way to generalize these constructions to other finite and affine Weyl groups.

Corollary 5.5. The elements $\mathbf{h}_{k}$ generate a commutative subalgebra of $\mathbb{A}_{0}$.

We call the subalgebra of Corollary 5.5 the Fomin-Stanley sublagebra of $\mathbb{A}_{0}$, and denote it by $\mathbb{B}$. As we shall explain, the combinatorics of Stanley symmetric functions is captured by the algebra $\mathbb{B}$, and the information can be extracted by "picking a basis". 


\subsection{A conjecture}

We take $W$ to be an arbitrary Weyl group. For basic facts concerning the exponents of $W$, we refer the reader to [64]. The following conjecture was made by the author and Alex Postnikov. Let $\left(R^{+}, \prec\right)$ denote the partial order on the positive roots of $W$ given by $\alpha \prec \beta$ if $\beta-\alpha$ is a positive sum of simple roots, and let $J\left(R^{+}, \prec\right)$ denote the set of upper order ideals of $\left(R^{+}, \prec\right)$.

Conjecture 5.6. The (finite) nilCoxeter algebra $\mathbb{A}_{0}$ contains a graded commutative subalgebra $\mathbb{B}^{\prime}$ satisfying:

1. Over the rationals, the algebra $\mathbb{B}^{\prime} \otimes_{\mathbb{Z}} \mathbb{Q}$ is generated by homogeneous elements $\mathbf{h}_{i_{1}}, \mathbf{h}_{i_{2}}, \ldots, \mathbf{h}_{i_{r}} \in$ $\mathbb{A}_{0}$ with degrees $\operatorname{deg}\left(\mathbf{h}_{i_{j}}\right)=i_{j}$ given by the exponents of $W$.

2. The Hilbert series $P(t)$ of $\mathbb{B}^{\prime}$ is given by

$$
P(t)=\sum_{I \in J\left(R^{+}, \prec\right)} t^{|I|}
$$

In particular, the dimension of $\mathbb{B}^{\prime}$ is a the generalized Catalan number for $W$ (see for example [47]).

3. The set $\mathbb{B}^{\prime}$ has a homogeneous basis $\left\{b_{I} \mid I \in J\left(R^{+}, \prec\right)\right\}$ consisting of elements which are nonnegative linear combinations of the $A_{w}$.

4. The structure constants of the basis $\left\{b_{I}\right\}$ are positive.

In the sequel we shall give an explicit construction of a commutative subalgebra $\mathbb{B}$ and provide evidence that it satisfies Conjecture 5.6.

Suppose $W=S_{n}$. We show that $\mathbb{B}^{\prime}=\mathbb{B}$ satisfies Conjecture 5.6. The upper order ideals $I$ of $\left(R^{+}, \prec\right)$ are naturally in bijection with Young diagrams fitting inside the staircase $\delta_{n-1}$. For each partition $\lambda$ we define the noncommutative Schur functions $\mathbf{s}_{\lambda} \in \mathbb{B}$, following Fomin and Greene [44], by writing $s_{\lambda}$ as a polynomial in the $h_{i}$, and then replacing $h_{i}$ by $\mathbf{h}_{i}$. (We set $\mathbf{h}_{k}=0$ if $k \geq n$, and $\mathbf{h}_{0}=1$.) Fomin and Greene show that $\mathbf{s}_{\lambda}$ is a nonnegative linear combination of the $A_{w}$ 's (but it will also follow from our use of the Edelman-Greene correspondence below).

Proposition 5.7 (Lam - Postnikov). The set $\left\{\mathbf{s}_{\lambda} \mid \lambda \subseteq \delta_{n-1}\right\} \subset \mathbb{B}$ is a basis for $\mathbb{B}$.

Proof. Let $\langle.,\rangle:. \mathbb{A}_{0} \otimes \mathbb{A}_{0} \rightarrow \mathbb{Z}$ be the inner product defined by extending bilinearly $\left\langle A_{w}, A_{v}\right\rangle=\delta_{w v}$. Rewriting the definition of Stanley symmetric functions and using the Cauchy identity, one has

$$
\begin{aligned}
F_{w} & =\sum_{\lambda}\left\langle\mathbf{h}_{\lambda_{1}} \mathbf{h}_{\lambda_{2}} \cdots, A_{w}\right\rangle m_{\lambda} \\
& =\sum_{\lambda}\left\langle\mathbf{s}_{\lambda}, A_{w}\right\rangle s_{\lambda} .
\end{aligned}
$$


It follows that the coefficient of $A_{w}$ in $\mathbf{s}_{\lambda}$ is $\alpha_{w \lambda}$, the coefficient of $s_{\lambda}$ in $F_{w}$. By Lemma 2.3 , we have $\mathbf{s}_{\lambda}=0$ unless $\lambda \subseteq \delta_{n-1}$. It remains to show that this set of $\mathbf{s}_{\lambda}$ are linearly independent. To demonstrate this, we shall find, for each $\lambda \in \delta_{n-1}$, some $w \in S_{n}$ such that $F_{w}=s_{\lambda}+\sum_{\mu \prec \lambda} \alpha_{w \mu} s_{\mu}$.

Since $s_{\lambda}=m_{\lambda}+\sum_{\mu \prec \lambda} K_{\lambda \mu} m_{\mu}$ (where the $K_{\lambda \mu}$ are the Kostka numbers), by Proposition 1.8 it suffices to show that the permutation $w$ with code $c(w)=\lambda$ lies in $S_{n}$. Let $\lambda=$ $\left(\lambda_{1} \geq \lambda_{2} \geq \ldots \geq \lambda_{\ell}>0\right)$. Then define $w$ recursively by $w(i)=\min \left\{j>\lambda_{i} \mid j \notin\right.$ $\{w(1), w(2), \ldots, w(i-1)\}$. Since $\lambda_{i} \leq n-i$, we have $w(i) \leq n$. By construction $w \in S_{n}$ and has code $c(w)=\lambda$.

Example 5.8. Let $W=S_{4}$. Write $A_{i_{1} i_{2} \cdots i_{k}}$ for $A_{s_{i_{1}} s_{i_{2}} \cdots s_{i_{k}}}$.

Then we have

$$
\begin{array}{ll}
\mathbf{s}_{\emptyset}=1 & \mathbf{s}_{1}=A_{1}+A_{2}+A_{3} \\
\mathbf{s}_{11}=A_{12}+A_{23}+A_{13} & \mathbf{s}_{2}=A_{21}+A_{32}+A_{31} \\
\mathbf{s}_{111}=A_{123} & \mathbf{s}_{3}=A_{321} \\
\mathbf{s}_{21}=A_{213}+A_{212}+A_{323}+A_{312} & \mathbf{s}_{211}=A_{1323}+A_{1213} \\
\mathbf{s}_{22}=A_{2132} & \mathbf{s}_{31}=A_{3231}+A_{3121} \\
\mathbf{s}_{221}=A_{23123} & \mathbf{s}_{311}=A_{32123} \\
\mathbf{s}_{32}=A_{32132} & \mathbf{s}_{321}=A_{321323}
\end{array}
$$

\subsection{Exercises and Problems}

1. In Example 5.8 every $A_{w}$ occurs exactly once, except for $w=s_{1} s_{3}$. Explain this using Theorem 2.5.

2. (Divided difference operators) Let $\partial_{i}, 1 \leq i \leq n-1$ denote the divided difference operator acting on polynomials in $x_{1}, x_{2}, \ldots, x_{n}$ by

$$
\partial_{i} \cdot f\left(x_{1}, \ldots, x_{n}\right)=\frac{f\left(x_{1}, x_{2}, \ldots, x_{n}\right)-f\left(x_{1}, \ldots, x_{i-1}, x_{i+1}, x_{i}, x_{i+2}, \ldots, x_{n}\right)}{x_{i}-x_{i+1}} .
$$

Show that $A_{i} \mapsto \partial_{i}$ generates an action of the nilCoxeter algebra $\mathbb{A}_{0}$ for $S_{n}$ on polynomials. Is this action faithful?

3. (Center) What is the center of $\mathbb{A}_{0}$ and of $\left(\mathbb{A}_{\mathrm{af}}\right)_{0}$ ? See [25].

4. Does an analogue of Conjecture 5.6 also hold for finite Coxeter groups which are not Weyl groups?

5. How many terms (counted with multiplicity) are there in the elements $\mathbf{s}_{\lambda}$ of Proposition 5.7? This is essentially the same problem as (9) in Section 2.3 (why?). 


\section{The affine nilHecke ring}

Kostant and Kumar [75] introduced a nilHecke ring to study the topology of Kac-Moody flag varieties. This ring is studied in detail in Chapter 4, Section 3. We give a brief development here. Let $W$ be a Weyl group and $W_{\text {af }}$ be the corresponding affine Weyl group.

\subsection{Definition of affine nilHecke ring}

In this section we study the affine nilHecke ring $\mathbb{A}_{\text {af }}$ of Kostant and Kumar [75]. Kostant and Kumar define the nilHecke ring in the Kac-Moody setting. The ring $\mathbb{A}_{\text {af }}$ below is a "small torus" variant of their construction for the affine Kac-Moody case.

The affine nilHecke ring $\mathbb{A}_{\mathrm{af}}$ is the ring with a 1 given by generators $\left\{A_{i} \mid i \in I_{\mathrm{af}}\right\} \cup\{\lambda \mid$ $\lambda \in P\}$ and the relations

$$
\begin{aligned}
A_{i} \lambda & =\left(s_{i} \cdot \lambda\right) A_{i}+\left\langle\alpha_{i}^{\vee}, \lambda\right\rangle \cdot 1 & & \text { for } \lambda \in P, \\
A_{i} A_{i} & =0, & & \\
\left(A_{i} A_{j}\right)^{m} & =\left(A_{j} A_{i}\right)^{m} & & \text { if }\left(s_{i} s_{j}\right)^{m}=\left(s_{j} s_{i}\right)^{m} .
\end{aligned}
$$

where the "scalars" $\lambda \in P$ commute with other scalars. Thus $\mathbb{A}_{\mathrm{af}}$ is obtained from the affine nilCoxeter algebra $\left(\mathbb{A}_{\mathrm{af}}\right)_{0}$ by adding the scalars $P$. The finite nilHecke ring is the subring $\mathbb{A}$ of $\mathbb{A}_{\text {af }}$ generated by $\left\{A_{i} \mid i \in I\right\} \cup\{\lambda \mid \lambda \in P\}$.

Let $w \in W_{\text {af }}$ and let $w=s_{i_{1}} \cdots s_{i_{l}}$ be a reduced decomposition of $w$. Then $A_{w}:=$ $A_{i_{1}} \cdots A_{i_{l}}$ is a well defined element of $\mathbb{A}_{\mathrm{af}}$, where $A_{\mathrm{id}}=1$.

Let $S=\operatorname{Sym}(P)$ denote the symmetric algebra of $P$. The following basic result follows from [75, Theorem 4.6], and can be proved directly from the definitions.

Lemma 6.1 (See Chapter 4, Lemma 3.6). The set $\left\{A_{w} \mid w \in W_{\mathrm{af}}\right\}$ is an S-basis of $\mathbb{A}_{\mathrm{af}}$.

Lemma 6.2. The map $W_{\mathrm{af}} \mapsto \mathbb{A}_{\mathrm{af}}$ given by $s_{i} \mapsto 1-\alpha_{i} A_{i} \in \mathbb{A}_{\mathrm{af}}$ is a homomorphism.

Proof. We calculate that

$$
\begin{aligned}
& s_{i}^{2}=1-2 \alpha_{i} A_{i}+\alpha_{i} A_{i} \alpha_{i} A_{i} \\
& =1-2 \alpha_{i} A_{i}+\alpha_{i}\left(-\alpha_{i} A_{i}+2\right) A_{i} \quad \text { using (6.1) } \\
& =1 \quad \text { using } A_{i}^{2}=0 \text {. }
\end{aligned}
$$

If $A_{i} A_{j}=A_{i} A_{j}$ then $\alpha_{i} A_{j}=A_{j} \alpha_{i}$ so it is easy to see that $s_{i} s_{j}=s_{j} s_{i}$. Suppose $A_{i} A_{j} A_{i}=$ 
$A_{j} A_{i} A_{j}$. Then $a_{i j}=a_{j i}=-1$ and we calculate that

$$
\begin{aligned}
s_{i} s_{j} s_{i}= & \left(1-\alpha_{i} A_{i}\right)\left(1-\alpha_{j} A_{j}\right)\left(1-\alpha_{i} A_{i}\right) \\
= & 1-\left(2 \alpha_{i} A_{i}+\alpha_{j} A_{j}\right)+\left(\alpha_{i} A_{i} \alpha_{j} A_{j}+\alpha_{i} A_{i} \alpha_{i} A_{i}+\alpha_{j} A_{j} \alpha_{i} A_{i}\right)-\alpha_{i} A_{i} \alpha_{j} A_{j} \alpha_{i} A_{i} \\
= & 1-\left(2 \alpha_{i} A_{i}+\alpha_{j} A_{j}\right)+\left(2 \alpha_{i} A_{j}+2 \alpha_{i} A_{i}+2 \alpha_{j} A_{i}\right)+\alpha_{i}\left(\alpha_{i}+\alpha_{j}\right) A_{i} A_{j}+ \\
& \alpha_{j}\left(\alpha_{i}+\alpha_{j}\right) A_{j} A_{i}-\left(\alpha_{i} A_{i}+\alpha_{i}\left(\alpha_{i}+\alpha_{j}\right) \alpha_{j} A_{i} A_{j} A_{i}\right) \\
= & 1+\left(2 \alpha_{i} A_{j}+2 \alpha_{j} A_{i}-\alpha_{i} A_{i}-\alpha_{j} A_{j}\right)+\alpha_{i}\left(\alpha_{i}+\alpha_{j}\right) A_{i} A_{j}+ \\
& \alpha_{j}\left(\alpha_{i}+\alpha_{j}\right) A_{j} A_{i}-\alpha_{i}\left(\alpha_{i}+\alpha_{j}\right) \alpha_{j} A_{i} A_{j} A_{i} .
\end{aligned}
$$

Since the above expression is symmetric in $i$ and $j$, we conclude that $s_{i} s_{j} s_{i}=s_{j} s_{i} s_{j}$.

Exercise 6.3. Complete the proof of Lemma 6.2 for $\left(A_{i} A_{j}\right)^{2}=\left(A_{j} A_{i}\right)^{2}$ and $\left(A_{i} A_{j}\right)^{3}=$ $\left(A_{j} A_{i}\right)^{3}$.

It follows from Lemma 6.1 that the map of Lemma 6.2 is an isomorphism onto its image. Abusing notation, we write $w \in \mathbb{A}_{\text {af }}$ for the element in the nilHecke ring corresponding to $w \in W_{\text {af }}$ under the map of Lemma 6.2. Then $W_{\text {af }}$ is a basis for $\mathbb{A}_{\mathrm{af}} \otimes_{S} \operatorname{Frac}(S) \operatorname{over} \operatorname{Frac}(S)$ (not over $S$ since $A_{i}=\frac{1}{\alpha_{i}}\left(1-s_{i}\right)$ ).

Lemma 6.4. Suppose $w \in W_{\text {af }}$ and $s \in S$. Then $w s=(w \cdot s) w$.

Proof. It suffices to establish this for $w=s_{i}$, and $s=\lambda \in P$. We calculate

$$
\begin{aligned}
s_{i} \lambda & =\left(1-\alpha_{i} A_{i}\right) \lambda \\
& =\lambda-\alpha_{i}\left(s_{i} \cdot \lambda\right) A_{i}-\alpha_{i}\left\langle\alpha_{i}^{\vee}, \lambda\right\rangle \\
& =\left(s_{i} \cdot \lambda\right)-\left(s_{i} \cdot \lambda\right) \alpha_{i} A_{i} \\
& =\left(s_{i} \cdot \lambda\right) s_{i} .
\end{aligned}
$$

\subsection{Coproduct}

The following Proposition is established in Chapter 4, Section 3.2. Note that the Weyl group $W$ there should be taken to be the affine Weyl group $W_{\text {af }}$ here.

Proposition 6.5. Let $M$ and $N$ be left $\mathbb{A}_{\text {af }}$-modules. Define

$$
M \otimes_{S} N=\left(M \otimes_{\mathbb{Z}} N\right) /\langle s m \otimes n-m \otimes s n \mid s \in S, m \in M, n \in N\rangle .
$$

Then $\mathbb{A}_{\mathrm{af}}$ acts on $M \otimes_{S} N$ by

$$
\begin{aligned}
s \cdot(m \otimes n) & =s m \otimes n \\
A_{i} \cdot(m \otimes n) & =A_{i} \cdot m \otimes n+m \otimes A_{i} \cdot n-\alpha_{i} A_{i} \cdot m \otimes A_{i} \cdot n .
\end{aligned}
$$


Under this action we have

$$
w \cdot(m \otimes n)=w m \otimes w n
$$

for any $w \in W_{\mathrm{af}}$.

Consider the case $M=\mathbb{A}_{\mathrm{af}}=N$. By Proposition 6.5 there is a left $S$-module homomorphism $\Delta: \mathbb{A}_{\mathrm{af}} \rightarrow \mathbb{A}_{\mathrm{af}} \otimes_{S} \mathbb{A}_{\mathrm{af}}$ defined by $\Delta(a)=a \cdot(1 \otimes 1)$. It satisfies

$$
\begin{aligned}
\Delta(q) & =s \otimes 1 & & \text { for } s \in S \\
\Delta\left(A_{i}\right) & =1 \otimes A_{i}+A_{i} \otimes 1-\alpha_{i} A_{i} \otimes A_{i} & & \text { for } i \in I .
\end{aligned}
$$

Let $a \in \mathbb{A}_{\mathrm{af}}$ and $\Delta(a)=\sum_{v, w} a_{v, w} A_{v} \otimes A_{w}$ with $a_{v, w} \in S$. In particular if $b \in \mathbb{A}_{\mathrm{af}}$ and $\Delta(b)=\sum_{v^{\prime}, w^{\prime}} b_{v^{\prime}, w^{\prime}} A_{v^{\prime}} \otimes A_{w^{\prime}}$ then

$$
\Delta(a b)=\Delta(a) \cdot \Delta(b):=\sum_{v, w, v^{\prime}, w^{\prime}} a_{v, w} b_{v^{\prime}, w^{\prime}} A_{v} A_{v^{\prime}} \otimes A_{w} A_{w^{\prime}}
$$

Remark 6.6. We caution that $\mathbb{A}_{\mathrm{af}} \otimes_{S} \mathbb{A}_{\mathrm{af}}$ is not a well-defined ring with the obvious multiplication.

\subsection{Exercises and Problems}

The theory of nilHecke rings in the Kac-Moody setting is well-developed [75, 77]. See Chapter 4, Section 3.

1. The following result is [75, Proposition 4.30]. Let $w \in W_{\text {af }}$ and $\lambda \in P$. Then

$$
A_{w} \lambda=(w \cdot \lambda) A_{w}+\sum_{w s_{\alpha}: \ell\left(w s_{\alpha}\right)=\ell(w)-1}\left\langle\alpha^{\vee}, \lambda\right\rangle A_{w s_{\alpha}}
$$

where $\alpha$ is always taken to be a positive root of $W_{\text {af }}$. The coefficients $\left\langle\alpha^{\vee}, \lambda\right\rangle$ are known as Chevalley coefficients.

2. (Center) What is the center of $\mathbb{A}_{\mathrm{af}}$ ? (See [79, Section 9] for related discussion.)

\section{Peterson's centralizer algebras}

Peterson studied a subalgebra of the affine nilHecke ring in his work [131] on the homology of the affine Grassmannian. 


\subsection{Peterson algebra and $j$-basis}

The Peterson centralizer subalgebra $\mathbb{P}$ is the centralizer $Z_{\mathbb{A}_{\mathrm{af}}}(S)$ of the scalars $S$ in the affine nilHecke ring $\mathbb{A}_{\mathrm{af}}$. In this section we establish some basic properties of this subalgebra. The results here are unpublished works of Peterson.

Lemma 7.1. Suppose $a \in \mathbb{A}_{\mathrm{af}}$. Write $a=\sum_{w \in W_{\mathrm{af}}} a_{w} w$, where $a_{w} \in \operatorname{Frac}(S)$. Then $a \in \mathbb{P}$ if and only if $a_{w}=0$ for all non-translation elements $w \neq t_{\lambda}$.

Proof. By Lemma 6.4, we have for $s \in S$

$$
\left(\sum_{w} a_{w} w\right) s=\sum_{w} a_{w}(w \cdot s) w
$$

and so $a \in \mathbb{P}$ implies $a_{w}(w \cdot s)=a_{w} s$ for each $s$. But using (4.1), one sees that every $w \in W_{\text {af }}$ acts non-trivially on $S$ except for the translation elements $t_{\lambda}$. Since $S$ is an integral domain, this implies that $a_{w}=0$ for all non-translation elements.

Lemma 7.2. The subalgebra $\mathbb{P}$ is commutative.

Proof. Follows from Lemma 7.1 and the fact that the elements $t_{\lambda}$ commute, and commute with $S$.

The following important result is the basis of Peterson's approach to affine Schubert calculus via the affine nilHecke ring.

Theorem 7.3 ([131, 79]). The subalgebra $\mathbb{P}$ has a basis $\left\{j_{w} \mid w \in W_{\text {af }}^{0}\right\}$ where

$$
j_{w}=A_{w}+\sum_{x \in W_{\mathrm{af}}-W_{\mathrm{af}}^{0}} j_{w}^{x} A_{x}
$$

for $j_{w}^{x} \in S$.

Peterson constructs the basis of Theorem 7.3 using the geometry of based loop spaces (see $[131,79])$. We sketch a purely algebraic proof of this theorem, following the ideas of Lam, Schilling, and Shimozono [85]. A full proof of this theorem is given in Chapter 4.

\subsection{Sketch proof of Theorem 7.3}

Let $\operatorname{Fun}\left(W_{\mathrm{af}}, S\right)$ denote the set of functions $\xi: W_{\mathrm{af}} \rightarrow S$. We may think of functions $\xi \in \operatorname{Fun}\left(W_{\mathrm{af}}, S\right)$ as functions on $\mathbb{A}_{\mathrm{af}}$, by the formula $\xi\left(\sum_{w \in W_{\mathrm{af}}} a_{w} w\right)=\sum_{w \in W_{\mathrm{af}}} \xi(w) a_{w}$. Note that if $a=\sum_{w \in W_{\mathrm{af}}} a_{w} w \in \mathbb{A}_{\mathrm{af}}$, the $a_{w}$ may lie in $\operatorname{Frac}(S)$ rather than $S$, so that in general $\xi(a) \in \operatorname{Frac}(S)$. Define

$$
\Xi_{\mathrm{af}}:=\left\{\xi \in \operatorname{Fun}\left(W_{\mathrm{af}}, S\right) \mid \xi(a) \in S \text { for all } a \in \mathbb{A}_{\mathrm{af}}\right\} .
$$


(The ring $\Xi_{\mathrm{af}}$ is denoted $\Lambda_{\mathrm{af}}$ in Chapter 4.) Let

$$
\Xi_{\mathrm{af}}^{0}=\left\{\xi \in \Xi_{\mathrm{af}} \mid \xi(w)=\xi(v) \text { whenever } w W=v W\right\} .
$$

It follows easily that $\Xi_{\text {af }}$ has a basis over $S$ given by $\left\{\xi^{w} \mid w \in W_{\text {af }}\right\}$ where $\xi^{w}\left(A_{v}\right)=\delta_{v w}$ for every $v \in W_{\text {af }}$. Similarly, $\Xi_{\text {af }}^{0}$ has a basis given by $\left\{\xi_{0}^{w} \mid w \in W_{\text {af }}^{0}\right\}$ where $\xi_{0}^{w}\left(A_{v}\right)=\delta_{v w}$ for every $v \in W_{\text {af }}^{0}$.

The most difficult step is the following statement:

Lemma 7.4. There is a map $\omega: \Xi_{\mathrm{af}} \rightarrow \Xi_{\mathrm{af}}^{0}$ defined by $\omega(\xi)\left(t_{\lambda}\right)=\xi\left(t_{\lambda}\right)$.

In other words, $\omega(\xi)$ remembers only the values of $\xi$ on translation elements, and one notes that $\omega\left(\xi^{w}\right)=\xi_{0}^{w}$ for $w \in W_{\mathrm{af}}^{0}$. By Lemma 7.1, $\xi(a)=\omega(\xi)(a)$ for $\xi \in \Xi_{\text {af }}$ and $a \in \mathbb{P}$. We define $\left\{j_{w} \in \mathbb{P} \otimes \operatorname{Frac}(S) \mid w \in W_{\text {af }}^{0}\right\}$ by the equation

$$
\xi_{0}^{v}\left(j_{w}\right)=\delta_{w v}
$$

for $w, v \in W_{\text {af }}^{0}$. Such elements $j_{w}$ exist (and $\operatorname{span} \mathbb{P} \otimes \operatorname{Frac}(S)$ over $\left.\operatorname{Frac}(S)\right)$ since each $\xi \in \Xi_{\mathrm{af}}^{0}$ is determined by its values on the translations $t_{\lambda}$. The coefficient $j_{w}^{x}$ of $A_{x}$ in $j_{w}$ is equal to the coefficient of $\xi_{0}^{w}$ in $\omega\left(\xi^{x}\right)$, which by Lemma 7.4, must lie in $S$. This proves Theorem 7.3.

Finally, Lemma 7.4 follows from the following description of $\Xi_{\mathrm{af}}$ and $\Xi_{\mathrm{af}}^{0}$, the latter due to Goresky-Kottwitz-Macpherson [58, Theorem 9.2]. See [85] for an algebraic proof in a slightly more general situation.

Proposition 7.5. Let $\xi \in \operatorname{Fun}\left(W_{\mathrm{af}}, S\right)$. Then $\xi \in \Xi_{\mathrm{af}}$ if and only if for each $\alpha \in R$, $w \in W_{\text {af }}$, and each integer $d>0$ we have

$$
\xi\left(w\left(1-t_{\alpha^{\vee}}\right)^{d-1}\right) \quad \text { is divisible by } \alpha^{d-1}
$$

and

$$
\xi\left(w\left(1-t_{\alpha^{\vee}}\right)^{d-1}\left(1-s_{\alpha}\right)\right) \quad \text { is divisible by } \alpha^{d} .
$$

Let $\xi \in \operatorname{Fun}\left(W_{\mathrm{af}}, S\right)$ satisfy $\xi(w)=\xi(v)$ whenever $w W=v W$. Then $\xi \in \Xi_{\mathrm{af}}^{0}$ if it satisfies (7.1).

Remark 7.6. The ring $\Xi_{\text {af }}$ is studied in detail by Kostant and Kumar [75] in the KacMoody setting. See Chapter 4.

\subsection{Exercises and Problems}

1. Show that $\Delta$ sends $\mathbb{P}$ to $\mathbb{P} \otimes \mathbb{P}$. Show that the coproduct structure constants of $\mathbb{P}$ in the $j$-basis are special cases of the coproduct structure constants of $\mathbb{A}_{\mathrm{af}}$ in the $A_{w^{-}}$basis. 
2. ( $j$-basis for translations $[131,79])$ Prove using Theorem 7.3 that

$$
j_{t_{\lambda}}=\sum_{\mu \in W \cdot \lambda} A_{t_{\mu}}
$$

3. ( $j$-basis is self-describing) Show that the coefficients $j_{w}^{x}$ directly determine the structure constants of the $\left\{j_{w}\right\}$ basis.

4. Find a formula for $j_{s_{i} t_{\lambda}}$ (see [88, Proposition 8.5] for a special case).

5. Extend the construction of $\mathbb{P}$, and Theorem 7.3 to extended affine Weyl groups. See [30] and [90, Theorem 12].

6. (Generators) Find generators and relations for $\mathbb{P}$. This does not appear to be known even in type $A$.

7. Find general formulae for $j_{w}$ in terms of $A_{x}$. See [88] for a formula in terms of quantum Schubert polynomials, which however is not very explicit.

\section{8 (Affine) Fomin-Stanley algebras}

Let $\phi_{0}: S \rightarrow \mathbb{Z}$ denote the map which sends a polynomial to its constant term. For example, $\phi_{0}\left(3 \alpha_{1}^{2} \alpha_{2}+\alpha_{2}+5\right)=5$.

\subsection{Commutation definition of affine Fomin-Stanley algebra}

We write $\left(\mathbb{A}_{\mathrm{af}}\right)_{0}$ for the affine nilCoxeter algebra. There is an evaluation at 0 map $\phi_{0}$ : $\mathbb{A}_{\mathrm{af}} \rightarrow\left(\mathbb{A}_{\mathrm{af}}\right)_{0}$ given by $\phi_{0}\left(\sum_{w} a_{w} A_{w}\right)=\sum_{w} \phi_{0}\left(a_{w}\right) A_{w}$. We define the affine FominStanley subalgebra to be $\mathbb{B}_{\mathrm{af}}=\phi_{0}(\mathbb{P}) \subset\left(\mathbb{A}_{\mathrm{af}}\right)_{0}$. The following results follow from Lemma 7.2 and Theorem 7.3.

Lemma 8.1. The set $\mathbb{B}_{\mathrm{af}} \subset\left(\mathbb{A}_{\mathrm{af}}\right)_{0}$ is a commutative subalgebra of $\left(\mathbb{A}_{\mathrm{af}}\right)_{0}$.

Theorem 8.2 ([79]). The algebra $\mathbb{B}_{\mathrm{af}}$ has a basis $\left\{j_{w}^{0} \mid w \in W_{\mathrm{af}}^{0}\right\}$ satisfying

$$
j_{w}^{0}=A_{w}+\sum_{\substack{x \notin W_{\mathrm{a}}^{0} \\ \ell(x)=\ell(w)}} j_{w}^{x} A_{x}
$$

and $j_{w}^{0}$ is the unique element in $\mathbb{B}_{\mathrm{af}}$ with unique Grassmanian term $A_{w}$.

Proposition 8.3 ([79]). The subalgebra $\mathbb{B}_{\mathrm{af}} \subset\left(\mathbb{A}_{\mathrm{af}}\right)_{0}$ is given by

$$
\mathbb{B}_{\mathrm{af}}=\left\{a \in\left(\mathbb{A}_{\mathrm{af}}\right)_{0} \mid \phi_{0}(a s)=\phi_{0}(s) a \text { for all } s \in S\right\} .
$$


Proposition 8.3 is proved in the following exercises (also see [79, Propositions 5.1,5.3,5.4]).

\section{Exercise 8.4.}

1. Check that $a \in \mathbb{B}_{\text {af }}$ satisfies $\phi_{0}(a s)=\phi_{0}(s) a$ for all $s \in S$, thus obtaining one inclusion.

2. Show that if $a=\sum_{w \in W} a_{w} A_{w} \in \mathbb{A}_{0}$ lies in $\mathbb{B}_{\mathrm{af}}$, then $a$ is a multiple of $A_{\mathrm{id}}$. (Hint: the action of $\mathbb{A}_{0}$ on $S$ via divided difference operators is faithful. See Section 5.4.)

3. Suppose that $a \in\left(\mathbb{A}_{\mathrm{af}}\right)_{0}$ satisfies the condition $\phi_{0}(a s)=\phi_{0}(s) a$ for all $s \in S$. Use (2) to show that $a$ must contain some affine Grassmannian term $A_{w}, w \in W_{\text {af }}^{0}$. Conclude by Theorem 8.2 that $a \in \mathbb{B}_{\text {af }}$.

A basic problem is to describe $\mathbb{B}_{\text {af }}$ explicitly. We shall do so in type $A$, following [79] and connecting to affine Stanley symmetric functions. For other types, see [84, 132].

\subsection{Noncommutative $k$-Schur functions}

In the remainder of this section, we take $W=S_{n}$ and $W_{\text {af }}=\tilde{S}_{n}$. Part of the material of this section is also presented in Chapter 2, Section 2.5. We define

$$
\tilde{\mathbf{h}}_{k}=\sum_{w \text { cyclically decreasing: } \ell(w)=k} A_{w}
$$

for $k=1,2, \ldots, n-1$. Introduce an inner product $\langle.,\rangle:.\left(\mathbb{A}_{\mathrm{af}}\right)_{0} \times\left(\mathbb{A}_{\mathrm{af}}\right)_{0} \rightarrow \mathbb{Z}$ given by extending linearly $\left\langle A_{w}, A_{v}\right\rangle=\delta_{w v}$.

Definition 8.5. The affine Stanley symmetric function $\tilde{F}_{w}$ is given by

$$
\tilde{F}_{w}=\sum_{\alpha}\left\langle h_{\alpha_{\ell}} \cdots h_{\alpha_{1}}, A_{w}\right\rangle x^{\alpha}
$$

where the sum is over compositions $\alpha=\left(\alpha_{1}, \alpha_{2}, \ldots, \alpha_{\ell}\right)$.

Below we shall show that

Theorem 8.6 ([79]). The elements $\tilde{\mathbf{h}}_{1}, \tilde{\mathbf{h}}_{2}, \ldots, \tilde{\mathbf{h}}_{n-1} \in\left(\mathbb{A}_{\mathrm{af}}\right)_{0}$ commute.

Assuming Theorem 8.6, Theorem 3.3 follows.

Define the noncommutative $k$-Schur functions $\mathbf{s}_{\lambda}^{(k)} \in\left(\mathbb{A}_{\mathrm{af}}\right)_{0}$ by writing the $k$-Schur functions $s_{\lambda}^{(k)}$ (see Section 3) as a polynomial in $h_{i}$, and replacing $h_{i}$ by $\tilde{\mathbf{h}}_{i}$.

Proposition 8.7 ([79]). Inside an appropriate completion of $\left(\mathbb{A}_{\mathrm{af}}\right)_{0} \otimes \Lambda^{(n)}$, we have

$$
\sum_{\alpha} \tilde{\mathbf{h}}_{\alpha} x^{\alpha}=\sum_{\lambda \in \mathcal{B}^{n}} \mathbf{s}_{\lambda}^{(k)} \tilde{F}_{\lambda}
$$

where the sum on the left hand side is over all compositions. 
Proof. Since $\left\{s_{\lambda}^{(k)}\right\}$ and $\left\{\tilde{F}_{\lambda}\right\}$ are dual bases, we have by standard results in symmetric functions $[151,120]$ that

$$
\sum_{\alpha} h_{\alpha}(y) x^{\alpha}=\sum_{\lambda \in \mathcal{B}^{n}} s_{\lambda}^{(k)}(y) \tilde{F}_{\lambda}(x)
$$

inside $\Lambda_{(n)} \otimes \Lambda^{(n)}$. Now take the image of this equation under the map $\Lambda_{(n)} \rightarrow\left(\mathbb{A}_{\mathrm{af}}\right)_{0}$, given by $h_{i} \mapsto \tilde{\mathbf{h}}_{i}$.

It follows from Definition 8.5 and Proposition 8.7 that

$$
\tilde{F}_{w}=\sum_{\lambda}\left\langle\mathbf{s}_{\lambda}^{(k)}, A_{w}\right\rangle \tilde{F}_{\lambda}
$$

Thus the coefficient of $A_{w}$ in $\mathbf{s}_{\lambda}^{(k)}$ is equal to the coefficient of $\tilde{F}_{\lambda}$ in $\tilde{F}_{w}$. By Theorem 3.7, it follows that

$$
\mathbf{s}_{\lambda}^{(k)}=A_{v}+\sum_{w \notin W_{\mathrm{af}}^{0}} \alpha_{w \lambda} A_{w} .
$$

In particular,

Proposition 8.8 ([79]). The subalgebra of $\left(\mathbb{A}_{\mathrm{af}}\right)_{0}$ generated by $\tilde{\mathbf{h}}_{1}, \ldots, \tilde{\mathbf{h}}_{n-1}$ is isomorphic to $\Lambda_{(n)}$, with basis given by $\mathbf{s}_{\lambda}^{(k)}$.

\subsection{Cyclically decreasing elements}

For convenience, for $I \subsetneq \mathbb{Z} / n \mathbb{Z}$ we define $A_{I}:=A_{w}$, where $w$ is the unique cyclically decreasing affine permutation which uses exactly the simple generators in $I$.

Theorem 8.9 ([79]). The affine Fomin-Stanley subalgebra $\mathbb{B}_{\text {af }}$ is generated by the elements $\tilde{\mathbf{h}}_{k}$, and we have $j_{w}^{0}=\mathbf{s}_{\lambda}^{(k)}$ where $w \in W_{\text {af }}^{0}$ satisfies $\lambda(w)=\lambda$.

Example 8.10. Let $W=S_{3}$. A part of the $j$-basis for $\mathbb{B}_{\mathrm{af}}$ is

$$
\begin{aligned}
j_{\text {id }}^{0} & =1 \\
j_{s_{0}}^{0} & =A_{0}+A_{1}+A_{2} \\
j_{s_{1} s_{0}}^{0} & =A_{10}+A_{21}+A_{02} \\
j_{s_{2} s_{0}}^{0} & =A_{01}+A_{12}+A_{20} \\
j_{s_{2} s_{1} s_{0}}^{0} & =A_{101}+A_{102}+A_{210}+A_{212}+A_{020}+A_{021} \\
j_{s_{1} s_{2} s_{0}}^{0} & =A_{101}+A_{201}+A_{012}+A_{212}+A_{020}+A_{120}
\end{aligned}
$$

We give a slightly different proof to the one in [79]. 
Proof. We begin by showing that $\tilde{\mathbf{h}}_{k} \in \mathbb{B}_{\text {af }}$. We will view $S$ as sitting inside the polynomial ring $\mathbb{Z}\left[x_{1}, x_{2}, \ldots, x_{n}\right]$; the commutation relations of $\mathbb{A}_{\text {af }}$ can easily be extended to include all such polynomials. To show that $\tilde{\mathbf{h}}_{k} \in \mathbb{B}_{\text {af }}$ it suffices to check that $\phi_{0}\left(\tilde{\mathbf{h}}_{k} x_{i}\right)=0$ for each $i$. But by the $\mathbb{Z} / n \mathbb{Z}$-symmetry of the definition of cyclically decreasing, we may assume $i=1$.

We note that

$$
A_{j} x_{i}= \begin{cases}x_{i+1} A_{i}+1 & j=i \\ x_{i-1} A_{i}-1 & j=i-1 \\ x_{i} A_{j} & \text { otherwise }\end{cases}
$$

Now let $I \subsetneq \mathbb{Z} / n \mathbb{Z}$ be a subset of size $k$. Then

$$
\phi_{0}\left(A_{I} x_{1}\right)= \begin{cases}A_{I \backslash\{1\}} & r, r+1, \ldots, n-1,0,1 \in I \text { but } r-1 \notin I \\ -\sum_{i=r}^{i=0} A_{I \backslash\{i\}} & r, r+1, \ldots, n-1,0 \in I \text { but } r-1,1 \notin I \\ 0 & \text { otherwise. }\end{cases}
$$

Given a size $k-1$ subset $J \subset \mathbb{Z} / n \mathbb{Z}$ not containing 1 , we see that the term $A_{J}$ comes up in two ways: from $\phi_{0}\left(A_{J \cup\{1\}} x_{1}\right)$ with a positive sign, and from $\phi_{0}\left(A_{J \cup\left\{r^{\prime}\right\}} x_{1}\right)$ with a negative sign, where $r+1, \ldots, n-1,0$ all lie in $J$.

Thus $\tilde{\mathbf{h}}_{k} \in \mathbb{B}_{\text {af }}$ for each $k$. It follows that the $\tilde{\mathbf{h}}_{k}$ commute, and by (8.1), it follows that $j_{w}^{0}=\mathbf{s}_{\lambda}^{(k)}$ and in particular $\tilde{\mathbf{h}}_{i}$ generate $\mathbb{B}_{\mathrm{af}}$.

\subsection{Coproduct}

The map $\Delta: \mathbb{A}_{\mathrm{af}} \rightarrow \mathbb{A}_{\mathrm{af}} \otimes_{S} \mathbb{A}_{\mathrm{af}}$ equips $\mathbb{P}$ with the structure of a Hopf-algebra over $S$ : to see that $\Delta$ sends $\mathbb{P}$ to $\mathbb{P} \otimes_{S} \mathbb{P}$, one uses $\Delta\left(t_{\lambda}\right)=t_{\lambda} \otimes t_{\lambda}$ and Lemma 7.1. Applying $\phi_{0}$, the affine Fomin-Stanley algebra $\mathbb{B}_{\text {af }}$ obtains a structure of a Hopf algebra over $\mathbb{Z}$.

Theorem 8.11 ([79]). The map $\Lambda_{(n)} \rightarrow \mathbb{B}_{\text {af }}$ given by $h_{i} \mapsto \tilde{\mathbf{h}}_{i}$ is an isomorphism of Hopf algebras.

By Proposition 8.8, to establish Theorem 8.11 it suffices to show that $\Delta\left(\tilde{\mathbf{h}}_{k}\right)=\sum_{j=0}^{k} \tilde{\mathbf{h}}_{j} \otimes$ $\tilde{\mathbf{h}}_{k-j}$. This can be done bijectively, using the definition in Section 6.2.

\subsection{Exercises and Problems}

Let $W$ be of arbitrary type.

1. Find a formula for $j_{s_{0}}^{0}$. See [87, Proposition 2.17].

2. For $W=S_{3}$, use Proposition 3.10 to give an explicit formula for the noncommutative $k$-Schur functions. 
3. (Dynkin automorphisms) Let $\omega$ be an automorphism of the corresponding Dynkin diagram. Then $\omega$ acts on $\left(\mathbb{A}_{\mathrm{af}}\right)_{0}$, and it is easy to see that $\omega\left(\mathbb{B}_{\mathrm{af}}\right)=\mathbb{B}_{\mathrm{af}}$. Is $\mathbb{B}_{\mathrm{af}}$ invariant under $\omega$ ? (For $W=S_{n}$ this follows from Theorem 8.9, since the $\tilde{\mathbf{h}}_{k}$ are invariant under cyclic symmetry.)

4. (Generators) Find generators and relations for $\mathbb{B}_{\text {af }}$, preferably using a subset of the $j$-basis as generators. See $[84,132]$ for the classical types. See also the proof of Proposition 9.4 below.

5. (Power sums [7, Corollary 3.7]) Define the noncommutative power sums $\mathbf{p}_{k} \in \mathbb{B}_{\text {af }}$ as the image in $\mathbb{B}_{\mathrm{af}}$ of $p_{k} \in \Lambda_{(n)}$ under the isomorphism of Proposition 8.8. Find an explicit combinatorial formula for $\mathbf{p}_{k}$. (See also [44].)

6. Is there a nice formula for the number of terms in the expression of $j_{w}^{0}$ in terms of $\left\{A_{x}\right\}$ ? This is an "affine nilCoxeter" analogue of asking for the number of terms $s_{\lambda}(1,1, \ldots, 1)$ in a Schur polynomial.

7. Find a combinatorial formula for the coproduct structure constants in the $j$-basis. These coefficients are known to be positive [77].

8. Find a combinatorial formula for the $j$-basis. It follows from work of Peterson (see also $[79,88])$ that the coefficients $j_{w}^{x}$ are positive.

\section{$9 \quad$ Finite Fomin-Stanley subalgebra}

In this section we return to general type.

There is a linear map $\kappa: \mathbb{A} \rightarrow\left(\mathbb{A}_{\mathrm{af}}\right)_{0}$ given by

$$
\kappa\left(A_{w}\right)= \begin{cases}A_{w} & w \in W \\ 0 & \text { otherwise }\end{cases}
$$

The finite Fomin-Stanley algebra $\mathbb{B}$ is the image of $\mathbb{P}$ under $\kappa$. Since $\kappa\left(\tilde{\mathbf{h}}_{k}\right)=\mathbf{h}_{k}$, this agrees with the definitions in Section 5.

\section{Conjecture 9.1.}

1. The finite Fomin-Stanley algebra $\mathbb{B}$ satisfies Conjecture 5.6.

2. The image $\kappa\left(j_{w}^{0}\right) \in \mathbb{B}$ of the $j$-basis element $j_{w}^{0} \in \mathbb{B}_{\text {af }}$ is a nonnegative integral linear combination of the $b_{I}$-basis.

3. If $w \in W_{\mathrm{af}}^{0}$ is such that there is a Dynkin diagram automorphism $\omega$ so that $\omega(w) \in W$, then $\kappa\left(j_{w}^{0}\right)$ belongs to the $b_{I}$-basis. 
Example 9.2. Let $W=S_{4}$ and $W_{\text {af }}=\tilde{S}_{4}$. Then $\mathbb{B}$ is described in Example 5.8. One calculates that $s_{221}^{(k)}=s_{221}+s_{32}$ so that

$$
\kappa\left(\mathbf{s}_{221}^{(k)}\right)=\mathbf{s}_{221}+\mathbf{s}_{32}=A_{32132}+A_{23123} \in \mathbb{B} .
$$

This supports Conjecture $9.1(2)$, and shows that $\kappa\left(j_{w}^{0}\right)$ does not have to be equal to 0 or to some $b_{I}$.

In the following we allow ourselves some geometric arguments and explicit calculations in other types to provide evidence for Conjecture 9.1 .

Example 9.3. Let $W=G_{2}$ with long root $\alpha_{1}$ and short root $\alpha_{2}$. Thus $\alpha_{0}$ is also a long root for $\tilde{G}_{2}$. As usual we shall write $A_{i_{1} i_{2} \cdots i_{\ell}}$ for $A_{s_{i_{1}} s_{i_{2}} \cdots s_{i_{\ell}}}$ and similarly for the $j$-basis. The affine Grassmannian elements of length less than or equal to 5 are

$$
\text { id, } s_{0}, s_{1} s_{0}, s_{2} s_{1} s_{0}, s_{1} s_{2} s_{1} s_{0}, s_{2} s_{1} s_{2} s_{1} s_{0}, s_{0} s_{1} s_{2} s_{1} s_{0} .
$$

And the $j$-basis is given by

$$
\begin{aligned}
j_{\text {id }}^{0} & =1 \\
j_{0}^{0} & =A_{0}+2 A_{1}+A_{2} \\
j_{10}^{0} & =\frac{1}{2}\left(j_{0}^{0}\right)^{2} \\
j_{210}^{0} & =\frac{1}{2}\left(j_{0}^{0}\right)^{3} \\
j_{1210}^{0} & =\frac{1}{4}\left(j_{0}^{0}\right)^{4} \\
j_{21210}^{0} & =A_{21210}+A_{21201}+2 A_{21012}+A_{12102}+3 A_{12101}+2 A_{12121} \\
& +A_{12012}+A_{02121}+3 A_{01201}+A_{01212} \\
j_{01210}^{0} & =A_{01210}+A_{01201}+A_{02121}+A_{12012}+A_{12101}+A_{12102}+A_{21012} \\
& +A_{21201}+A_{21212}
\end{aligned}
$$

which can be verified by using Theorem 8.2. Note that $j_{21210}^{0}+j_{01210}^{0}=\frac{1}{4}\left(j_{0}^{0}\right)^{5}$. Thus $\mathbb{B}$ has basis

id, $2 A_{1}+A_{2}, A_{12}+A_{21}, 2 A_{121}+A_{212}, A_{1212}+A_{2121}, 2 A_{12121}, A_{21212}, ? A_{121212}$

where the coefficient of $A_{121212}$ depends on the $j$-basis in degree 6 . The root poset of $G_{2}$ is $\alpha_{1}, \alpha_{2} \prec \alpha_{1}+\alpha_{2} \prec \alpha_{1}+2 \alpha_{2} \prec \alpha_{1}+3 \alpha_{2} \prec 2 \alpha_{1}+3 \alpha_{2}$. Both Conjectures 5.6 and 9.1 hold with this choice of basis.

Proposition 9.4. Over the rationals, the finite Fomin-Stanley subalgebra $\mathbb{B} \otimes_{\mathbb{Z}} \mathbb{Q}$ has a set of generators in degrees equal to the exponents of $W$. 
Proof. Let $\mathrm{Gr}_{G}$ denote the affine Grassmannian of the simple simply-connected complex algebraic group with Weyl group $W$. It is known [79, Theorem 5.5] that $\mathbb{B}_{\mathrm{af}} \simeq H_{*}\left(\mathrm{Gr}_{G}, \mathbb{Z}\right)$. But over the rationals the homology $H_{*}\left(\mathrm{Gr}_{G}, \mathbb{Q}\right)$ of the affine Grassmannian is known to be generated by elements in degrees equal to the exponents of $W$, see [54]. (In cohomology these generators are obtained by transgressing generators of $H^{*}(K, \mathbb{Q})$ which are known to correspond to the degrees of $W$.) Since these elements generate $\mathbb{B}_{\mathrm{af}}$, their image under $\kappa$ generate $\mathbb{B}$.

\subsection{Problems}

1. Find a geometric interpretation for the finite Fomin-Stanley algebras $\mathbb{B}$ and the conjectural basis $b_{I}$.

2. Find an equivariant analogue of the finite Fomin Stanley algebra $\mathbb{B}$, with the same relationship to $\mathbb{B}$ as $\mathbb{P}$ has to $\mathbb{B}_{\text {af. }}$. Extend Conjectures 5.6 and 9.1 to the equivariant setting.

\section{Geometric interpretations}

In this section we list some geometric interpretations of the material we have discussed. Let $G$ be the simple simply-connected complex algebraic group associated to $W$. (Co)homologies are with $\mathbb{Z}$-coefficients.

1. The affine Fomin-Stanley subalgebra $\mathbb{B}_{\text {af }}$ is Hopf-isomorphic to the homology $H_{*}\left(\operatorname{Gr}_{G}\right)$ of the affine Grassmannian associated to $G$. The $j$-basis $\left\{j_{w}\right\}$ is identified with the Schubert basis. [131] [79, Theorem 5.5]

2. The affine Schur functions $\tilde{F}_{\lambda}$ represent Schubert classes in $H^{*}\left(\operatorname{Gr}_{S L(n)}\right)$. [79, Theorem 7.1]

3. The affine Stanley symmetric functions are the pullbacks of the cohomology Schubert classes from the affine flag variety to the affine Grassmannian. [79, Remark 8.6]

4. The positivity of the affine Stanley to affine Schur coefficients is established via the connection between the homology of the $\mathrm{Gr}_{G}$ and the quantum cohomology of $G / B$. [131] [88] [116]

5. The (positive) expansion of the affine Schur symmetric functions $\left\{\tilde{F}_{\lambda}\right\}$ for $W_{\text {af }}=$ $\tilde{S}_{n}$ in terms of $\left\{\tilde{F}_{\mu}\right\}$ for $W_{\text {af }}=\tilde{S}_{n-1}$ has an interpretation in terms of the map $H^{*}(\Omega S U(n+1)) \rightarrow H^{*}(\Omega S U(n))$ induced by the inclusion $\Omega S U(n) \hookrightarrow \Omega S U(n+1)$ of based loop spaces. [80]

6. Certain affine Stanley symmetric functions represent the classes of positroid varieties in the cohomology of the Grassmannian. [73] 
7. The expansion coefficients of Stanley symmetric functions in terms of Schur functions are certain quiver coefficients. [26] 


\title{
Chapter 4
}

\section{Affine Schubert calculus}

\author{
MARK SHIMOzono ${ }^{1}$ \\ mshimo@math.vt.edu
}

\section{Introduction}

This chapter discusses how $k$-Schur and dual $k$-Schur functions can be defined for all types. This is done via some combinatorial problems that come from the geometry of a very large family of generalized flag varieties. They apply to the expansion of products of Schur functions, $k$-Schur functions and their dual basis, and Schubert polynomials. Despite the geometric origin of these problems, concrete algebraic models will be given for the relevant cohomology rings and their Schubert bases.

Let $G \supset P \supset T$ be a Kac-Moody group over $\mathbb{C}$ [77], a parabolic subgroup, and a maximal algebraic torus. For example, we may take $G=G L_{n}(\mathbb{C}), P$ the block upper triangular matrices with diagonal blocks of sizes $k$ and $n-k$, and $T \subset G$ the diagonal matrices. The group $G$ may be specified by a graph called a Dynkin diagram, plus a little more discrete data called a root datum; see $\S 2$. The subgroup $P$ is obtained by choosing a subset $J$ of the vertex set of the Dynkin diagram. Examples of $G / P$ are the Grassmannian $\operatorname{Gr}(k, n)$ of $k$-dimensional subspaces of $\mathbb{C}^{n}$, the variety $\mathrm{Fl}_{n}$ of full flags in $\mathbb{C}^{n}$, and the affine Grassmannian $\operatorname{Gr}_{S L_{n}}=S L_{n}(\mathbb{C}((t))) / S L_{n}(\mathbb{C}[[t]])$ where $\mathbb{C}[[t]]$ is the ring of formal power series and $\mathbb{C}((t))=\mathbb{C}[[t]]\left[t^{-1}\right]$ is the field of formal Laurent series. Given this modest amount of discrete data (root datum and Dynkin node subset) one may build the $T$-equivariant cohomology ring $H^{T}(G / P)$ of $G / P$. Following Kostant and Kumar [75] we shall give an explicit algebraic construction $\Lambda^{J}$ of $H^{T}(G / P)$; see $\S 3.6$. The ring $H^{T}(G / P)$ is a free module over the polynomial ring $S=H^{T}(p t)$, and comes equipped with a distinguished basis $\left\{\left[X^{w}\right]\right\}$ called the Schubert basis, which is indexed by a certain subset

\footnotetext{
${ }^{1}$ The author was supported by NSF grants DMS-0652641, DMS-0652648, and DMS-1200804.
} 
$W^{J}$ of the Weyl group $W$ :

$$
H^{T}(G / P)=\bigoplus_{w \in W^{J}} S\left[X^{w}\right]
$$

For $u, v, w \in W^{J}$ define the "structure constants" $p_{u v}^{w} \in S$ by

$$
\left[X^{u}\right]\left[X^{v}\right]=\sum_{w \in W^{J}} p_{u v}^{w}\left[X^{w}\right]
$$

For nontrivial geometric reasons it is known that the polynomial $p_{u v}^{w}$ is homogeneous of degree $\ell(u)+\ell(v)-\ell(w)$ and satisfies a property called Graham positivity [59]; see Section $\S 3.4$.

Problem 1.1. Find explicit combinatorial formulas for $p_{u v}^{w}$ which are obviously Grahampositive.

Using the algebraic model $\Lambda^{J}$, there is an effective algorithm for expanding any element of $H^{T}(G / P)$ into the Schubert basis, and particular for computing the polynomials $p_{u v}^{w}$.

Ordinary cohomology $H^{*}(G / P)$ can be recovered by forgetting equivariance, which means setting all the variables in the polynomial ring $S$ to zero. Consequently the Schubert structure constants for $H^{*}(G / P)$ are merely the polynomials $p_{u v}^{w}$ which are of degree zero, in which Graham-positivity reduces to the nonnegativity of an integer. Special cases of these nonnegative integers are the structure constants for the products of Schur functions (in the case of $\operatorname{Gr}(k, n)$ ), dual $k$-Schur functions (for $\operatorname{Gr}_{S L_{n}}$ ), and Schubert polynomials (for $\mathrm{Fl}_{n}$ ).

To understand $H^{T}(G / P)$ and its Schubert calculus, it suffices to consider the case that $P=B$ is the Borel subgroup. Kostant and Kumar defined a ring of operators $\mathbb{A}$ on $H^{T}(G / B)$ called the nilHecke ring, which is a noncommutative ring which is linearly dual (over $S$ ) with $H^{T}(G / B)$. Following Kostant and Kumar, we develop the algebraic construction $\Lambda$ of $H^{T}(G / B)$ via the systematic use of $\mathbb{A}$.

The above machinery is then specialized to the case of the affine Grassmannian Gr. In this setting the equivariant cohomology ring $H^{T}(\mathrm{Gr})$ has the rich additional structure of a Hopf algebra, with Hopf dual given by the equivariant homology ring $H_{T}(\mathrm{Gr})$ under the Pontryagin product. In this context, we explain Peterson's theory, which asserts the equality of the Schubert structure constants $d_{u v}^{w}$ for equivariant homology $H_{T}(\mathrm{Gr})$ with the Gromov-Witten invariants (quantum Schubert structure constants) for finite-dimensional flag varieties $G / B$. As a special case we obtain Lam's noncommutative $k$-Schur functions [79] and the relationship between $k$-Schur functions and the quantum Schubert polynomials of Fomin, Gelfand, and Postnikov [43]. We give a general explicit formula (Proposition 4.11) for the coefficients $d_{u v}^{w}$ which involves only some computations in $H^{T}(\mathrm{Gr})$ (localizing Schubert classes) that we already know how to do explicitly from the general machinery for $H^{T}(G / P)$. 


\section{Root Data}

We specify the data with which one may construct a Kac-Moody Lie algebra $\mathfrak{g}$ [67], KacMoody group $G$ [77], flag ind-variety Fl [77], thick flag scheme Fl [68], and nilHecke algebra [75]. Affine and finite root systems are treated briefly in Chapter 3 Section 4; see also Chapter 4 Section 2.3.

\subsection{Cartan Data and the Weyl group}

A Cartan datum is a pair $(I, A)$ where $I$ is a set and $A=\left(a_{i j}\right)_{i, j \in I}$ is a generalized Cartan matrix, that is, an $I \times I$ matrix of integers such that $a_{i i}=2$ for all $i \in I$ and if $i \neq j$ then $a_{i j} \leq 0$ with $a_{i j}<0$ if and only if $a_{j i}<0$. A Cartan datum may be specified by a graph called a Dynkin diagram which has node set $I$ and an edge joining $i \in I$ and $j \in I$ if $a_{i j}<0$, in which case the edge is labeled by the pair $\left(-a_{i j},-a_{j i}\right)$. There are some abbreviations. Suppose $a_{i j}=-1$. If $a_{j i}=-1$ then the edge joining $i$ and $j$ is a plain single edge. If $a_{j i}=-2$ then there is a double edge pointing from $i$ to $j$. If $a_{j i}=-3$ then there is a triple edge pointing from $i$ to $j$.

\begin{tabular}{|c|c|c|c|}
\hline$\left(a_{i j}, a_{j i}\right)$ & $(-1,-1)$ & $(-1,-2)$ & $(-1,-3)$ \\
\hline al & $i-j$ & $i \quad \Longrightarrow j$ & $i \Longrightarrow j$ \\
\hline
\end{tabular}

A Cartan datum is finite if $A$ is positive definite; irreducible if the Dynkin diagram is connected; affine if it is irreducible, $A$ has corank 1 , and the restriction of $A$ to $I^{\prime} \times I^{\prime}$ for every proper subset $I^{\prime} \subset I$, yields a finite Cartan datum.

Given a Cartan datum $(I, A)$, the Weyl group $W=W(I, A)$ is the group by elements $\left\{s_{i} \mid i \in I\right\}$ called simple reflections with relations

$$
\begin{aligned}
s_{i}^{2} & =1 \\
\underbrace{s_{i} s_{j} s_{i} \cdots}_{m_{i j} \text { times }} & =\underbrace{s_{j} s_{i} s_{j} \cdots}_{m_{i j} \text { times }} .
\end{aligned}
$$

for $i \neq j$ where $m_{i j}$ and $a_{i j} a_{j i}$ are related by:

\begin{tabular}{|c||c|c|c|c|c|}
\hline$a_{i j} a_{j i}$ & 0 & 1 & 2 & 3 & $>3$ \\
\hline$m_{i j}$ & 2 & 3 & 4 & 6 & $\infty$ \\
\hline
\end{tabular}

The length $\ell(w)$ of $w \in W$ is the minimum $\ell$ such that $w=s_{i_{1}} s_{i_{2}} \cdots s_{i_{\ell}}$ for some indices $i_{1}, i_{2}, \ldots, i_{\ell} \in I$. Such a factorization is called a reduced decomposition for $w$. A reflection in $W$ is an element of the form $w s_{i} w^{-1}$ for some $w \in W$ and $i \in I$. The Bruhat order $\leq$ on $W$ is the partial order generated by relations of the form $t w<w$ where $t$ is a reflection and $w \in W$ are such that $\ell(t w)<\ell(w)$. It is also generated by relations $w t<w$ where 
$\ell(w t)<\ell(w)$. Equivalently $v \leq w$ if every (or equivalently some) reduced decomposition of $w$ contains a subexpression that is a reduced decomposition for $v$. We denote by $v \lessdot w$ the covering relation for $W$. It is equivalent to saying that $v<w$ and $\ell(w)=\ell(v)+1$.

Example 2.1. The Cartan datum of finite type $A_{n-1}$ is defined by the Dynkin diagram

$$
1 \quad 2 \quad \cdots-n-2 \quad n-1
$$

That is, $I=\{1,2, \ldots, n-1\}, a_{i i}=2$ for $1 \leq i \leq n-1, a_{i, i+1}=a_{i+1, i}=-1$ for $1 \leq i \leq n-2$, with $a_{i j}=0$ for other $(i, j) \in I \times I$. The Weyl group is the symmetric group $S_{n}$ with $s_{i}=(i, i+1)$ the adjacent transposition. The reflections are the transpositions $(i, j)$ for $1 \leq i<j \leq n$.

Example 2.2. A Cartan datum of finite type $C_{n}$ is given by the Dynkin diagram

$$
1-2-n-n
$$

In particular $a_{n-1, n}=-2$ and $a_{n, n-1}=-1$. $W$ is the hyperoctahedral group, which is isomorphic to the subgroup of $S_{2 n}$ generated by $s_{i}=(i, i+1)(2 n-i, 2 n-i+1)$ for $1 \leq i \leq n-1$ and $s_{n}=(n, n+1)$.

Example 2.3. A Cartan datum of affine type $A_{n-1}^{(1)}$ is given by the Dynkin diagram

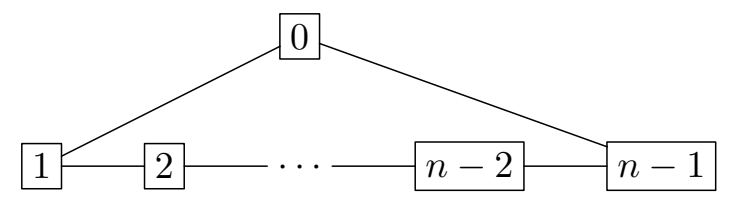

with Dynkin node set $I_{\mathrm{af}}=\mathbb{Z} / n \mathbb{Z}, a_{i i}=2$ for all $i \in I_{\mathrm{af}}, a_{i, i+1}=a_{i+1, i}=-1$ for all $i \in I_{\text {af }}$, including $a_{n-1,0}=a_{0, n-1}=-1 . W=\tilde{S}_{n}$ is the affine symmetric group. There is an injective homomorphism from $\tilde{S}_{n}$ into the permutations of $\mathbb{Z}$ such that $s_{i}$ is sent to the permutation of $\mathbb{Z}$ that exchanges $(i+k n, i+k n+1)$ for all $k \in \mathbb{Z}$.

Example 2.4. A Cartan datum of affine type $C_{n}^{(1)}$ is given by the Dynkin diagram

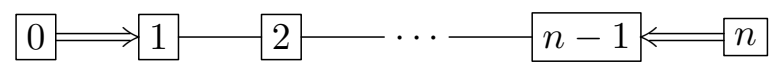

\subsection{Root data}

A root datum $\left(I, A, X, X^{*},\left\{\alpha_{i}\right\},\left\{\alpha_{i}^{\vee}\right\}\right)$ consists of a Cartan datum $(I, A)$ together with a free $\mathbb{Z}$-module $X$, the dual lattice $X^{*}=\operatorname{Hom}_{\mathbb{Z}}(X, \mathbb{Z})$, linearly independent elements $\left\{\alpha_{i} \mid i \in I\right\} \subset X$ called simple roots, linearly independent elements $\left\{\alpha_{i}^{\vee} \mid i \in I\right\} \subset X^{*}$ called simple coroots, such that

$$
\left\langle\alpha_{i}^{\vee}, \alpha_{j}\right\rangle=a_{i j} \quad \text { for } i, j \in I
$$


where $\langle\cdot, \cdot\rangle: X^{*} \times X \rightarrow \mathbb{Z}$ is the evaluation pairing.

A root datum must satisfy

$$
\operatorname{rank}(X) \geq|I|+\operatorname{nullity}(A) .
$$

A root datum is centerless if equality holds in (2.5).

$W$ acts on $X$ and $X^{*}$ via

$$
\begin{array}{ll}
s_{i} \cdot \lambda=\lambda-\left\langle\alpha_{i}^{\vee}, \lambda\right\rangle \alpha_{i} & \text { for } i \in I, \lambda \in X \\
s_{i} \cdot \mu=\mu-\left\langle\mu, \alpha_{i}\right\rangle \alpha_{i}^{\vee} & \text { for } i \in I, \mu \in X^{*} .
\end{array}
$$

These restrict to actions on the root lattice $Q$ and the coroot lattice $Q^{\vee}$ :

$$
\begin{gathered}
Q=\bigoplus_{i \in I} \mathbb{Z} \alpha_{i} \subset X \\
Q^{\vee}=\bigoplus_{i \in I} \mathbb{Z} \alpha_{i}^{\vee} \subset X^{*}
\end{gathered}
$$

One may show that

$$
\langle w \mu, w \lambda\rangle=\langle\mu, \lambda\rangle \quad \text { for } w \in W, \lambda \in X, \text { and } \mu \in X^{*} .
$$

The set of real roots is

$$
\Phi_{\mathrm{re}}=W \cdot\left\{\alpha_{i} \mid i \in I\right\} \subset X
$$

A real root

$$
\alpha=w \cdot \alpha_{i} \in \Phi_{\mathrm{re}}
$$

has an associated coroot

$$
\alpha^{\vee}=w \cdot \alpha_{i}^{\vee} \in X^{*}
$$

and a reflection

$$
s_{\alpha}=w s_{i} w^{-1} \in W .
$$

Both $\alpha^{\vee}$ and $s_{\alpha}$ are independent of the choices of $i$ and $w$. The reflection $s_{\alpha}$ acts on $X$ and $X^{*}$ by

$$
\begin{array}{ll}
s_{\alpha} \cdot \lambda=\lambda-\left\langle\alpha^{\vee}, \lambda\right\rangle \alpha & \text { for } \lambda \in X \\
s_{\alpha} \cdot \mu=\mu-\langle\mu, \alpha\rangle \alpha^{\vee} & \text { for } \mu \in X^{*} .
\end{array}
$$


For any $\alpha \in \Phi_{\text {re }}$ and $w \in W$, we have that

$$
\begin{aligned}
\beta & :=w \cdot \alpha \in \Phi_{\mathrm{re}} \\
\beta^{\vee} & =w \cdot \alpha^{\vee} \\
s_{\beta} & =w s_{\alpha} w^{-1} .
\end{aligned}
$$

The set of positive real roots is $\Phi_{\mathrm{re}}^{+}=\Phi_{\mathrm{re}} \cap \bigoplus_{i \in I} \mathbb{Z}_{\geq 0} \alpha_{i}$. One may show that

$$
\Phi_{\mathrm{re}}=\Phi_{\mathrm{re}}^{+} \cup-\Phi_{\mathrm{re}}^{+} \text {. }
$$

The inversion set of $w$ is

$$
\operatorname{Inv}(w)=\Phi_{\mathrm{re}}^{+} \cap w^{-1}\left(-\Phi_{\mathrm{re}}^{+}\right) \quad \text { for } w \in W .
$$

It is the set of positive roots which are sent to negative roots by $w$.

Lemma 2.5. Let $w \in W$. Then

$$
\begin{aligned}
\operatorname{Inv}(w) & =\left\{\alpha \in \Phi_{\mathrm{re}}^{+} \mid w s_{\alpha}<w\right\} \\
& =\left\{\alpha^{(j)}=s_{i_{1}} s_{i_{2}} \cdots s_{i_{j-1}} \cdot \alpha_{i_{j}} \mid 1 \leq j \leq \ell\right\} \quad \text { where } \\
w & =s_{i_{\ell}} \cdots s_{i_{1}} \quad \text { is a reduced decomposition. }
\end{aligned}
$$

Moreover none of the elements of $\operatorname{Inv}(w)$ is a scalar multiple of another.

Now for some details on root data. Let

$$
\begin{aligned}
L(Q) & =\left\{\lambda \in \mathbb{Q} \otimes_{\mathbb{Z}} X \mid\left\langle Q^{\vee}, \lambda\right\rangle \subset \mathbb{Z}\right\} \\
L\left(Q^{\vee}\right) & =\left\{\mu \in \mathbb{Q} \otimes_{\mathbb{Z}} X^{*} \mid\langle\mu, Q\rangle \subset \mathbb{Z}\right\}
\end{aligned}
$$

be the weight and coweight lattices. We have

$$
\begin{gathered}
Q \subset X \subset L(Q) \\
Q^{\vee} \subset X^{*} \subset L\left(Q^{\vee}\right)
\end{gathered}
$$

where $L(Q) / X$ and $L\left(Q^{\vee}\right) / X^{*}$ are finite. A root datum is simply connected if it is centerless and $L(Q)=X$.

Suppose the root datum is simply connected. Then $X$ has a basis $\left\{\omega_{i} \mid i \in I\right\} \cup\left\{\delta_{i} \mid\right.$ $1 \leq i \leq \operatorname{nullity}(A)\}$ and $X^{*}$ has a dual basis $\left\{\alpha_{i}^{\vee} \mid i \in I\right\} \cup\left\{d_{i} \mid 1 \leq i \leq \operatorname{nullity}(A)\right\}$. The $\omega_{i}$ are called fundamental weights and are uniquely defined. We have

$$
\begin{gathered}
\bigoplus_{i=1}^{\operatorname{nullity}(A)} \mathbb{Z} \delta_{i}=\left\{\lambda \in X \mid\left\langle Q^{\vee}, \lambda\right\rangle=0\right\}=X^{W} \\
\bigoplus_{i=1}^{\operatorname{nullity}(A)} \mathbb{Z} d_{i}=\left\{\mu \in X^{*} \mid\langle\mu, Q\rangle=0\right\}=X^{* W} .
\end{gathered}
$$


Since $\alpha_{k} \in X$ we have

$$
\alpha_{k}=\sum_{j \in I} a_{j k} \omega_{j} \bmod X^{W}
$$

Example 2.6. We give several root data for the type $A_{n-1}$ Cartan datum of Example 2.1. Recall that $I=\{1,2, \ldots, n-1\}$.

Let $\mathbb{Z}^{n}=\bigoplus_{i=1}^{n} \mathbb{Z} e_{i}$ be the standard basis and $x_{i} \in \operatorname{Hom}\left(\mathbb{Z}^{n}, \mathbb{Z}\right)$ be defined by $x_{i}\left(e_{j}\right)=$ $\delta_{i j}$ for $1 \leq i, j \leq n$. Let $\alpha_{i}=e_{i}-e_{i+1}$ and $\alpha_{i}^{\vee}=x_{i}-x_{i+1}$ for $i \in I$. These will be the simple roots and coroots, possibly up to taking suitable cosets. We have $\Phi_{\mathrm{re}}^{+}=\left\{\alpha_{i j}=\right.$ $\left.e_{i}-e_{j} \mid 1 \leq i<j \leq n\right\}$, and the associated reflection for $\alpha=\alpha_{i j}$ is the transposition $s_{\alpha}=(i, j)$.

1. The $G L_{n}$ root datum is given by taking $X=\mathbb{Z}^{n}$ and $X^{*}=\operatorname{Hom}\left(\mathbb{Z}^{n}, \mathbb{Z}\right)$. This root datum is not centerless: $\operatorname{nullity}(A)=0$ and $X^{W}=\mathbb{Z}\left(1^{n}\right)$.

2. The simply connected root datum is given by taking $X=\mathbb{Z}^{n} / \mathbb{Z}\left(1^{n}\right)=L(Q)$ and $X^{*}=Q^{\vee}$. Then $\omega_{i}=\left(1^{i}, 0^{n-i}\right)+\mathbb{Z}\left(1^{n}\right)$ for $i \in I$.

3. Let $x=x_{1}+x_{2}+\cdots+x_{n}$. The adjoint root datum is given by $X=Q$ and $X^{*}=\operatorname{Hom}\left(\mathbb{Z}^{n}, \mathbb{Z}\right) / \mathbb{Z} x=L\left(Q^{\vee}\right)$.

Example 2.7. Consider the type $C_{n}$ Cartan datum of Example 2.2. Using $X=\mathbb{Z}^{n}$ and $e_{i}$ and $x_{i}$ as in Example 2.6 we have $\alpha_{i}=e_{i}-e_{i+1}$ and $\alpha_{i}^{\vee}=x_{i}-x_{i+1}$ for $1 \leq i \leq n-1$, $\alpha_{n}=2 e_{n}$, and $\alpha_{n}^{\vee}=x_{n}$.

Remark 2.8. The fundamental coweights are elements $\omega_{i}^{\vee} \in \mathbb{Q} \otimes_{\mathbb{Z}} X^{*}$ such that

$$
\left\langle\omega_{i}^{\vee}, \alpha_{j}\right\rangle=\delta_{i j}
$$

for $i, j \in I$. The fundamental coweights need not be in the lattice $X^{*}$. In Example 2.7, we have $\omega_{n}^{\vee}=(1 / 2, \ldots, 1 / 2) \notin X^{*}$.

\subsection{Affine root data}

Let $\left(I, A, X, X^{*},\left\{\alpha_{i}\right\},\left\{\alpha_{i}^{\vee}\right\}\right)$ be an irreducible finite simply connected root datum. We shall describe the associated "untwisted" simply connected irreducible affine root datum denoted by $\left(I_{\mathrm{af}}, A_{\mathrm{af}}, X_{\mathrm{af}}, X_{\mathrm{af}}^{*},\left\{\alpha_{i}\right\},\left\{\alpha_{i}^{\vee}\right\}\right)$.

There is a distinguished node $0 \in I_{\text {af }}$ such that $I_{\text {af }}=I \cup\{0\}$ [67] and the restriction of $A_{\text {af }}$ to $I \times I$ is $A$. We write $W_{\text {af }}=W\left(I_{\text {af }}, A_{\text {af }}\right)$ for the affine Weyl group. There is a unique tuple $\left(a_{i} \mid i \in I_{\mathrm{af}}\right)$ of relatively prime positive integers which are the coefficients of a linear dependence relation among the columns of $A_{\mathrm{af}}$ :

$$
\sum_{j \in I} a_{j} a_{i j}=0 \quad \text { for all } i \in I_{\mathrm{af}} .
$$


The null root is

$$
\delta=\sum_{i \in I} a_{i} \alpha_{i}
$$

It is the unique generator of

$$
\begin{aligned}
X_{\mathrm{af}}^{W_{\mathrm{af}}} & =\left\{\lambda \in X_{\mathrm{af}} \mid w \cdot \lambda=\lambda \text { for all } w \in W_{\mathrm{af}}\right. \\
& =\left\{\lambda \in X_{\mathrm{af}} \mid\left\langle Q_{\mathrm{af}}^{\vee}, \lambda\right\rangle=0\right\}
\end{aligned}
$$

that lies in $\bigoplus_{i \in I_{\mathrm{af}}} \mathbb{Z}_{\geq 0} \alpha_{i}$. It satisfies

$$
\delta=\alpha_{0}+\theta
$$

where $\theta \in \Phi^{+}$is the highest root of the finite root datum.

There is a unique tuple $\left(a_{i}^{\vee} \mid i \in I_{\mathrm{af}}\right)$ of relatively prime positive integers which are the coefficients of a linear dependence relation among the rows of $A_{\mathrm{af}}$. The canonical central element $c \in X_{\mathrm{af}}^{*}$ is

$$
c=\sum_{i \in I_{\mathrm{af}}} a_{i}^{\vee} \alpha_{i}^{\vee}
$$

it generates

$$
\begin{aligned}
X_{\mathrm{af}}^{* W_{\mathrm{af}}} & =\left\{\mu \in X_{\mathrm{af}}^{*} \mid w \cdot \mu=\mu \text { for all } w \in W_{\mathrm{af}}\right\} \\
& =\left\{\mu \in X_{\mathrm{af}}^{*} \mid\left\langle\mu, Q_{\mathrm{af}}\right\rangle=0\right\} .
\end{aligned}
$$

We have

$$
c=\alpha_{0}^{\vee}+\theta^{\vee}
$$

where $\theta^{\vee} \in Q^{\vee}$ is the coroot associated to $\theta$.

We denote the affine fundamental weights by $\left\{\Lambda_{i} \mid i \in I_{\text {af }}\right\}$ and those of the finite root datum by $\left\{\omega_{i} \mid i \in I\right\}$. Then

$$
X_{\mathrm{af}}=\mathbb{Z} \delta \oplus \bigoplus_{i \in I_{\mathrm{af}}} \mathbb{Z} \Lambda_{i}
$$

and there is an element $d \in X_{\text {af }}^{*}$ called the degree generator, defined uniquely $\bmod \mathbb{Z} c$, such that $X_{\mathrm{af}}^{*}$ has dual basis

$$
X_{\mathrm{af}}^{*}=\mathbb{Z} d \oplus \bigoplus_{i \in I_{\mathrm{af}}} \mathbb{Z} \alpha_{i}^{\vee} .
$$


Then

$$
\alpha_{i}=\delta_{i 0}+\sum_{k \in I_{\mathrm{af}}} a_{k i} \Lambda_{k}
$$

where $A_{\mathrm{af}}=\left(a_{i j}\right)_{i, j \in I_{\mathrm{af}}}$. There is an exact sequence

$$
0 \rightarrow \mathbb{Z} \delta \oplus \mathbb{Z} \Lambda_{0} \rightarrow X_{\text {af }} \rightarrow X \rightarrow 0 .
$$

There is a section $X \rightarrow X_{\text {af }}$ defined by $\omega_{i} \mapsto \Lambda_{i}-a_{i}^{\vee} \Lambda_{0}$ for $i \in I$. Using this section we regard $Q \subset X \subset X_{\text {af }}$.

The real affine roots $\Phi_{\mathrm{af}}^{\mathrm{re}}$ and the positive subset $\Phi_{\mathrm{af}}^{\mathrm{re}+}$ are related to the set $\Phi$ of finite roots by

$$
\begin{aligned}
\Phi_{\mathrm{af}}^{\mathrm{re}} & =\Phi+\mathbb{Z} \delta \\
\Phi_{\mathrm{af}}^{\mathrm{re}+} & =\Phi^{+} \cup\left(\Phi+\mathbb{Z}_{>0} \delta\right) .
\end{aligned}
$$

Since $\left\langle c, Q_{\mathrm{af}}\right\rangle=0\left(\right.$ resp. $\left.\left\langle Q_{\mathrm{af}}^{\vee}, \delta\right\rangle=0\right)$, the action of $W_{\mathrm{af}}$ on $X_{\mathrm{af}}\left(\right.$ resp. $\left.X_{\mathrm{af}}^{*}\right)$ preserves the set of weights of a given level (resp. colevel) where

$$
\begin{aligned}
\operatorname{level}(\lambda) & =\langle c, \lambda\rangle & & \text { for } \lambda \in X_{\mathrm{af}} \\
\operatorname{colevel}(\mu) & =\langle\mu, \delta\rangle & & \text { for } \mu \in X_{\mathrm{af}}^{*} .
\end{aligned}
$$

Therefore for every $m \in \mathbb{Z}$ there is an action of $W_{\text {af }}$ called the level (colevel) $m$ action, on the set of elements of $X_{\mathrm{af}}$ of level zero (elements of $X_{\mathrm{af}}^{*}$ of colevel zero) which may be identified with $\mathbb{Z} \delta \oplus X$ (resp. $Q_{\mathrm{af}}^{\vee}=\mathbb{Z} c \oplus Q^{\vee}$ ), defined by

$$
\begin{array}{llrl}
w \cdot m & =-m \Lambda_{0}+w \cdot\left(m \Lambda_{0}+\lambda\right) & & \text { for } \lambda \in \mathbb{Z} \delta \oplus X \\
w \cdot m \mu & =-m d+w \cdot(m d+\mu) & & \text { for } \mu \in \mathbb{Z} c \oplus Q^{\vee} .
\end{array}
$$

For $\lambda \in \mathbb{Z} \delta \oplus X$ we have

$$
\begin{aligned}
& s_{i} \cdot{ }_{m} \lambda=s_{i} \cdot \lambda \quad \text { if } i \in I \\
& s_{0} \cdot{ }_{m} \lambda=s_{0} \cdot \lambda-m \alpha_{0} .
\end{aligned}
$$

For $\mu \in Q_{\text {af }}^{\vee}$ we have

$$
\begin{aligned}
& s_{i} \cdot m \mu=s_{i} \cdot \mu \quad \text { if } i \in I \\
& s_{0} \cdot m \mu=s_{0} \cdot \mu-m \alpha_{0}^{\vee} .
\end{aligned}
$$

Since

$$
\begin{aligned}
& W_{\mathrm{af}} \cdot \delta=\delta \\
& W_{\mathrm{af}} \cdot c=c
\end{aligned}
$$


the level $m$ action of $W_{\text {af }}$ on $\mathbb{Z} \delta \oplus X$ (resp. $Q_{\mathrm{af}}^{\vee}$ ) factors through projection $\bmod \mathbb{Z} \delta$ (resp. $\mathbb{Z} c$ ). So there is a level $m$ action of $W_{\text {af }}$ on $X$ given by

$$
\begin{aligned}
& s_{i} \cdot{ }_{m} \lambda=s_{i} \cdot \lambda \quad \text { for } i \in I \\
& s_{0} \cdot m \lambda=s_{\theta} \cdot \lambda+m \theta
\end{aligned}
$$

for $\lambda \in X$, and a colevel $m$ action of $W_{\text {af }}$ on $Q^{\vee}$ defined by

$$
\begin{aligned}
& s_{i} \cdot{ }_{m} \mu=s_{i} \cdot \mu \quad \text { if } i \in I \\
& s_{0} \cdot m \mu=s_{\theta} \cdot \mu+m \theta^{\vee}
\end{aligned}
$$

for $\mu \in Q^{\vee}$. Identifying $W_{\text {af }}$ with its image in $\operatorname{Aut}\left(Q^{\vee}\right)$ under the faithful level 1 action, we have

$$
W_{\mathrm{af}}=W \ltimes Q^{\vee}
$$

where $Q^{\vee}=\left\{t_{\mu} \mid \mu \in Q^{\vee}\right\}$ is the group of translations by elements of $Q^{\vee}$. We have

$$
w t_{\mu} w^{-1}=t_{w \cdot \mu} \quad \text { for } w \in W \text { and } \mu \in Q^{\vee} .
$$

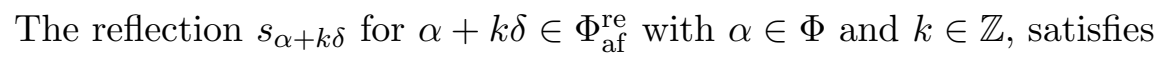

$$
s_{\alpha+k \delta}=s_{\alpha} t_{k \alpha} \text {. }
$$

In particular, for $\alpha_{0}=\delta-\theta$, we have

$$
s_{0}=s_{\theta} t_{-\theta^{\vee}} .
$$

Let $\mu \in Q^{\vee}$. Under the level $m$ action on $\mathbb{Z} \delta \oplus X$ we have

$$
t_{\mu} \cdot m(\lambda+k \delta)=(\lambda+k \delta)-(m+\langle\mu, \lambda\rangle) \delta \quad \text { for } \lambda \in X .
$$

Modding out by $\mathbb{Z} \delta$, the level 0 action of $W_{\text {af }}$ on $X$ is given by

$$
\left(u t_{\mu}\right){ }_{0} \lambda=u \cdot \lambda \quad \text { for } u \in W \text { and } \mu \in Q^{\vee} \text {; }
$$

the translations act trivially. Equivalently, by $(2.51), s_{0}$ acts by $s_{\theta}$.

Example 2.9. With the affine $A_{n-1}^{(1)}$ Cartan datum of Example 2.3, we have $a_{i}=1$ for all $i \in I_{\mathrm{af}}, \delta=\alpha_{0}+\alpha_{1}+\cdots+\alpha_{n-1}$ and $\theta=e_{1}-e_{n}$.

Example 2.10. With the affine $C_{2}^{(1)}$ Cartan datum of Example 2.4, we have $I_{\text {af }}=\{0,1,2\}$,

$$
A_{\mathrm{af}}=\left(\begin{array}{ccc}
2 & -1 & 0 \\
-2 & 2 & -2 \\
0 & -1 & 2
\end{array}\right)
$$

$\left(a_{0}, a_{1}, a_{2}\right)=(1,2,1), \delta=\alpha_{0}+2 \alpha_{1}+\alpha_{2}$, and $\theta=2 \alpha_{1}+\alpha_{2}$. We let $X=\mathbb{Z}^{2}, \alpha_{1}=(1,-1)$, $\alpha_{2}=(0,2), X^{*}=\mathbb{Z}^{2}, \alpha_{1}^{\vee}=(1,-1), \alpha_{2}^{\vee}=(0,1)$. Then $\theta=(2,0)=s_{1} \cdot \alpha_{2}$ so that $\theta^{\vee}=s_{1} \cdot \alpha_{2}^{\vee}=(1,0)=\alpha_{1}^{\vee}+\alpha_{2}^{\vee}$. So $c=\alpha_{0}^{\vee}+\alpha_{1}^{\vee}+\alpha_{2}^{\vee}$. 


\section{$3 \quad$ NilHecke ring and Schubert calculus}

In this section we consider the nilHecke ring $\mathbb{A}$ of Kostant and Kumar [75], for any KacMoody root datum. This general construction may be used to compute the cohomological Schubert calculus of the flag variety for the given root datum, equivariant with respect to the maximal torus.

The general construction of the Kostant-Kumar nilHecke ring $\mathbb{A}$ of this section, yields the nilHecke ring denoted $\mathbb{A}$ in Chapter 3 if a finite root datum is used, and yields an affine nilHecke ring $\mathbb{A}_{\mathrm{af}}$ if an untwisted affine root datum is used. This affine nilHecke ring $\mathbb{A}_{\mathrm{af}}$ is not the one denoted $\mathbb{A}_{\mathrm{af}}$ in Chapter 3; the latter, which we shall call $\mathbb{A}_{\text {af }}^{\prime}$, is a subquotient of $\mathbb{A}_{\mathrm{af}}$. Both compute cohomology of the affine flag variety, but $\mathbb{A}_{\mathrm{af}}$ uses equivariance for the maximal torus $T_{\text {af }}$ in the Kac-Moody group $G_{\text {af }}$, whereas $\mathbb{A}_{\text {af }}^{\prime}$ uses equivariance for the "small torus" $T$ in the simple Lie group $G \subset G_{\text {af }}$. See Sections 4.1 and 4.4.

The nilHecke ring $\mathbb{A}$ contains a subring $\mathbb{A}_{0}$ called the nilCoxeter algebra. For a finite root datum, $\mathbb{A}_{0}$ is the finite nilCoxeter algebra, also denoted $\mathbb{A}_{0}$ in Chapter 3 , Section 5 . For an untwisted affine root datum is of affine type, $\mathbb{A}_{0}$ is the affine nilCoxeter algebra, denoted $\left(\mathbb{A}_{\mathrm{af}}\right)_{0}$ in Chapter 3, Sections 5, 6.1, and 8.1.

\subsection{NilHecke ring}

Fix a root datum. Let $S=H^{T}(\mathrm{pt}) \cong \operatorname{Sym}(X)$ be the polynomial ring having a variable for each free generator of $X$ and let $\mathrm{F}=\operatorname{Frac}(S)$ be the fraction field. The action of $W$ on $X$ induces actions of $W$ on $S$ and $\mathrm{F}$ by ring automorphisms, and $\mathrm{F}$ acts on itself by left multiplication.

Example 3.1. For $A_{n-1}$ and the $G L_{n}$ root datum we have $X=\bigoplus_{i=1}^{n} \mathbb{Z} z_{n}$ and $S=$ $\mathbb{Z}\left[z_{1}, \ldots, z_{n}\right], \mathrm{F}=\mathbb{Q}\left(z_{1}, \ldots, z_{n}\right) . W=S_{n}$ permutes variables.

Let $v \in W$ and $q, q^{\prime} \in \mathrm{F}$. Viewing both as operators on $\mathrm{F}$ we have

$$
\begin{aligned}
(v \circ q) \cdot q^{\prime} & =v \cdot\left(q \cdot q^{\prime}\right) \\
& =(v \cdot q)\left(v \cdot q^{\prime}\right) \\
& =((v \cdot q) \circ v) \cdot q^{\prime},
\end{aligned}
$$

that is, $v q=(v \cdot q) v$ as operators on F. Define

$$
\mathrm{F}_{W}=\bigoplus_{w \in W} \mathrm{~F} w
$$

the F-vector space with basis $W$, with product given by

$$
(p v)(q w)=(p(v \cdot q))(v w) \quad \text { for } p, q \in \mathrm{F} \text { and } v, w \in W .
$$

It is the smash product of $\mathbb{Q}[W]$ and $\mathrm{F}$, the ring generated by the actions of $W$ and $\mathrm{F}$ upon F. 
Example 3.2. For the $A_{n-1}$ root datum of Example 2.6, we have $S=\mathbb{Z}\left[x_{1}, \ldots, x_{n}\right]$ and $\mathrm{F}=\mathbb{Q}\left(x_{1}, \ldots, x_{n}\right)$.

For any $\alpha \in \Phi_{\text {re }}$ define the element $A_{\alpha} \in \mathrm{F}_{W}$ by

$$
A_{\alpha}=\alpha^{-1}\left(1-s_{\alpha}\right) \text {. }
$$

We write

$$
A_{i}=A_{\alpha_{i}} \quad \text { for } i \in I \text {. }
$$

For $\alpha=w \cdot \alpha_{i} \in \Phi_{\text {re }}$ we have

$$
\begin{aligned}
w A_{i} w^{-1} & =A_{\alpha} \\
s_{\alpha} A_{\alpha} & =A_{\alpha} \\
A_{\alpha} s_{\alpha} & =-A_{\alpha} \\
A_{\alpha}^{2} & =0 .
\end{aligned}
$$

The $A_{i}$ satisfy the braid relations as the $s_{i}$ in $W$ :

$$
\underbrace{A_{i} A_{j} \cdots}_{m_{i j} \text { times }}=\underbrace{A_{j} A_{i} \cdots}_{m_{i j} \text { times. }}
$$

Therefore it makes sense to define

$$
\begin{aligned}
A_{w} & =A_{i_{1}} \cdots A_{i_{\ell}} & & \text { where } \\
w & =s_{i_{1}} \cdots s_{i_{\ell}} & & \text { is a reduced decomposition. }
\end{aligned}
$$

Using (3.8) and (3.9) one may show that

$$
A_{v} A_{w}= \begin{cases}A_{v w} & \text { if } \ell(v w)=\ell(v)+\ell(w) \\ 0 & \text { otherwise. }\end{cases}
$$

Since $A_{w} \in \mathrm{F}_{W}$ there are unique $c_{w, v} \in \mathrm{F}$ such that

$$
A_{w}=\sum_{v \in W} c_{w, v} v
$$

\section{Lemma 3.3.}

$$
\begin{aligned}
& c_{w, w} \neq 0 \\
& c_{w, v}=0 \quad \text { unless } w \geq v .
\end{aligned}
$$

In particular $\left\{A_{w} \mid w \in W\right\}$ is a left $\mathrm{F}$-basis of $\mathrm{F}_{W}$. 
The subring $\mathbb{A}_{0}$ of $\mathrm{F}_{W}$ generated by $\left\{A_{i} \mid i \in I\right\}$ is called the nilCoxeter algebra.

\section{Lemma 3.4.}

$$
\mathbb{A}_{0}=\bigoplus_{w \in W} \mathbb{Z} A_{w}
$$

Lemma 3.5. For every $\alpha \in \Phi_{\mathrm{re}}, \lambda \in X$, and $q, q^{\prime} \in S$, we have

$$
\begin{aligned}
A_{\alpha} \cdot \lambda & =\left\langle\alpha^{\vee}, \lambda\right\rangle \\
A_{\alpha} \cdot\left(q q^{\prime}\right) & =\left(A_{\alpha} \cdot q\right) q^{\prime}+\left(s_{\alpha} \cdot q\right)\left(A_{\alpha} \cdot q^{\prime}\right) .
\end{aligned}
$$

In particular, $A_{\alpha} \cdot S \subset S$ for all $\alpha \in \Phi_{\mathrm{re}}$.

The nilHecke ring $\mathbb{A}$ is the subring of $\mathrm{F}_{W}$ generated by $S$ and $\mathbb{A}_{0}$.

Lemma 3.6. $\mathbb{A}$ is a free left $S$-module with basis $\left\{A_{w} \mid w \in W\right\}$ :

$$
\mathbb{A}=\bigoplus_{w \in W} S A_{w}
$$

Proof. By (3.17) we have the commutation relation in $\mathbb{A}$ :

$$
A_{\alpha} q=\left(A_{\alpha} \cdot q\right) A_{\text {id }}+\left(s_{\alpha} \cdot q\right) A_{\alpha} \quad \text { for all } \alpha \in \Phi_{\text {re }} \text { and } q \in S .
$$

This relation may be used to commute all $A_{i}$ to the right past elements of $S$. This shows that $\left\{A_{w} \mid w \in W\right\}$ generates $\mathbb{A}$ as a left $S$-module. By Lemma 3.3 the $A_{w}$ are independent.

Let $v \lessdot w$ indicate a Bruhat covering relation: $v<w$ and $\ell(w)=\ell(v)+1$. In this case there is a unique $\alpha \in \Phi_{\mathrm{re}}^{+}$such that $w=v s_{\alpha}$.

Proposition 3.7. For any $v \in W$ and $\lambda \in X$, we have

$$
A_{v} \lambda=(v \cdot \lambda) A_{v}+\sum_{\substack{\alpha \in \Phi_{\mathrm{re}}^{+} \\ v s_{\alpha} \lessdot v}}\left\langle\alpha^{\vee}, \lambda\right\rangle A_{v s_{\alpha}} .
$$

Exercise 3.8. Prove Lemmas 3.3 and 3.5 and Proposition 3.7.

We have

$$
s_{i}=1-\alpha_{i} A_{i} \quad \text { for all } i \in I
$$

Therefore

$$
W \subset \mathbb{A}
$$


By Lemma 3.6 there exist unique elements $d_{v w} \in S$ such that

$$
w=\sum_{v \in W} d_{v w} A_{v}
$$

By (3.14) it follows that

$$
d_{v w}=0 \quad \text { unless } v \leq w .
$$

Lemma 3.9. The $d_{v, w}$ are uniquely defined by:

1. If $w=\mathrm{id}$ then

$$
d_{v, \mathrm{id}}=\delta_{v, \mathrm{id}}
$$

2. Otherwise let $i \in I$ be such that $w s_{i}<w$. Then

$$
d_{v, w}=d_{v, w s_{i}}+\chi\left(v s_{i}<v\right)\left(w \cdot \alpha_{i}\right) d_{v s_{i}, w s_{i}} .
$$

where

$$
\begin{aligned}
\chi(\text { true }) & =1 \\
\chi(\text { false }) & =0
\end{aligned}
$$

Proof. The uniqueness holds by induction on $w$ and then on $v$. Equation (3.25) holds since $\operatorname{id}_{W}=A_{\text {id }}$. We have

$$
\begin{aligned}
w & =\left(w s_{i}\right) s_{i} \\
& =\left(w s_{i}\right)\left(1-\alpha_{i} A_{i}\right) \\
& =w s_{i}-w s_{i} \alpha_{i} A_{i} \\
& =w s_{i}+\left(w \cdot \alpha_{i}\right) w s_{i} A_{i} .
\end{aligned}
$$

Taking the coefficient of $A_{v}$ on both sides and using (3.11) we obtain (3.26).

Proposition 3.10. Let $w=s_{i_{1}} \cdots s_{i_{\ell}}$ be a reduced decomposition. We have

$$
(-1)^{\ell(v)} d_{v, w}=\sum_{\substack{\left(b_{1}, \ldots, b_{\ell}\right) \in\{0,1\}^{\ell} \\ \prod_{j=1}^{\ell} A_{i_{j}}^{b_{j}}=A_{v}}}\left(\prod_{j=1}^{\ell} \alpha_{i_{j}}^{b_{j}} s_{i_{j}}\right) \cdot 1 .
$$

The parenthesized expression is viewed as an element of $\mathrm{F}_{W}$ acting on $1 \in \mathrm{F}$ and factors in the products occur in order from left to right as the index $j$ increases. 
Note that the sum runs over subexpressions of the given reduced decomposition of $w$, which are reduced decompositions of $v$.

Example 3.11. For type $A_{2}$ (symmetric group $S_{3}$ ) let $v=s_{1}$ and $w=s_{1} s_{2} s_{1}$. The reduced decomposition $s_{1} s_{2} s_{1}$ has two embedded copies of $s_{1}$ corresponding to the bit sequences $\left(b_{1}, b_{2}, b_{3}\right)$ given by $(1,0,0)$ and $(0,0,1)$. Therefore (3.29) has two summands:

$$
\begin{aligned}
(-1)^{\ell\left(s_{1}\right)} d_{s_{1}, s_{1} s_{2} s_{1}} & =\left(\left(\alpha_{1} s_{1}\right) s_{2} s_{1}+s_{1} s_{2}\left(\alpha_{1} s_{1}\right)\right) \cdot 1 \\
& =\alpha_{1}+s_{1} s_{2} \alpha_{1}=\alpha_{1}+\alpha_{2} .
\end{aligned}
$$

Here is a table of the values of $(-1)^{\ell(v)} d_{v, w}$ for all $v, w \in S_{3}$.

\begin{tabular}{|c||c|c|c|c|c|c|}
\hline$v \backslash w$ & 123 & 132 & 213 & 231 & 312 & 321 \\
\hline \hline 123 & 1 & 1 & 1 & 1 & 1 & 1 \\
\hline 132 & 0 & $\alpha_{2}$ & 0 & $\alpha_{1}+\alpha_{2}$ & $\alpha_{2}$ & $\alpha_{1}+\alpha_{2}$ \\
\hline 213 & 0 & 0 & $\alpha_{1}$ & $\alpha_{1}$ & $\alpha_{1}+\alpha_{2}$ & $\alpha_{1}+\alpha_{2}$ \\
\hline 231 & 0 & 0 & 0 & $\alpha_{1}\left(\alpha_{1}+\alpha_{2}\right)$ & 0 & $\alpha_{1}\left(\alpha_{1}+\alpha_{2}\right)$ \\
\hline 312 & 0 & 0 & 0 & 0 & $\alpha_{2}\left(\alpha_{1}+\alpha_{2}\right)$ & $\alpha_{2}\left(\alpha_{1}+\alpha_{2}\right)$ \\
\hline 321 & 0 & 0 & 0 & 0 & 0 & $\alpha_{1} \alpha_{2}\left(\alpha_{1}+\alpha_{2}\right)$ \\
\hline
\end{tabular}

Exercise 3.12. Prove Proposition 3.10.

Corollary 3.13. We have $(-1)^{\ell(v)} d_{v, w} \in \mathbb{Z}_{\geq 0}\left[\alpha_{i} \mid i \in I\right]$.

\subsection{Coproduct on $\mathbb{A}$}

The nilHecke ring $\mathbb{A}$ has a coproduct structure. This discussion follows Peterson [131] and Kostant and Kumar [75].

We first define the coproduct on $\mathrm{F}_{W}$ over $\mathrm{F}$.

Let $M$ and $N$ be left $\mathrm{F}_{W}$-modules. Then $M, N, M \otimes_{\mathrm{F}} N$, and $\operatorname{Hom}_{\mathrm{F}}(M, N)$ are left F-modules. We define $\mathrm{F}_{W}$-module structures on $M \otimes_{\mathrm{F}} N$ and $\operatorname{Hom}_{\mathrm{F}}(M, N)$.

Recall that $M \otimes_{\mathrm{F}} N$ has relations of left F-linearity in each factor, along with the relation

$$
q \cdot m \otimes n=m \otimes q \cdot n
$$

for all $q \in \mathrm{F}, m \in M$, and $n \in N$.

Let $\Delta: \mathrm{F}_{W} \rightarrow \mathrm{F}_{W} \otimes_{\mathrm{F}} \mathrm{F}_{W}$ be the left F-linear map defined by

$$
\Delta(w)=w \otimes w \quad \text { for all } w \in W .
$$

Clearly $\Delta$ has image $\mathrm{F}_{W}^{\prime}$, the left $\mathrm{F}$-subspace of $\mathrm{F}_{W} \otimes_{\mathrm{F}} \mathrm{F}_{W}$ defined by

$$
\mathrm{F}_{W}^{\prime}=\bigoplus_{w \in W} \mathrm{~F} w \otimes w
$$


It is obvious that $\mathrm{F}_{W}^{\prime}$ is isomorphic to $\mathrm{F}_{W}$ as a ring under the obvious componentwise product given by $(p v \otimes v)(q w \otimes w)=p v q w \otimes v w=p(v \cdot q) v w \otimes v w$.

The proof of the following result appears in Appendix A.

\section{Proposition 3.14.}

1. For any expressions of $\Delta(a)$ and $\Delta(b)$ in $\mathrm{F}_{W} \otimes_{\mathrm{F}} \mathrm{F}_{W}$ of the form

$$
\begin{aligned}
& \Delta(a)=\sum_{(a)} a_{(1)} \otimes a_{(2)} \\
& \Delta(b)=\sum_{(b)} b_{(1)} \otimes b_{(2)}
\end{aligned}
$$

with $a_{(1)}, a_{(2)}, b_{(1)}, b_{(2)} \in \mathrm{F}_{W}$ (but it is not assumed that the individual summands $a_{(1)} \otimes a_{(2)}$ or $b_{(1)} \otimes b_{(2)}$ are in $\left.\mathrm{F}_{W}^{\prime}\right)$, the product of $\Delta(a)$ and $\Delta(b)$ in $\mathrm{F}_{W}^{\prime}$ can be computed by the naive componentwise product

$$
\Delta(a b)=\Delta(a) \Delta(b)=\sum_{(a),(b)} a_{(1)} b_{(1)} \otimes a_{(2)} b_{(2)} .
$$

2. Suppose $M$ and $N$ are $\mathrm{F}_{W}$-modules. With $a \in \mathrm{F}_{W}$ and $\Delta(a)$ as above, $\mathrm{F}_{W}$ acts on $M \otimes_{\mathrm{F}} N$ by the componentwise action of $\Delta(a)$ on $m \otimes n:$

$$
a \cdot(m \otimes n)=\sum_{(a)} a_{(1)} \cdot m \otimes a_{(2)} \cdot n
$$

3. $\mathrm{F}_{W}$ acts on $\operatorname{Hom}_{\mathrm{F}}(M, N)$ by

$$
(a \cdot f)(m)=\sum_{(a)} a_{(2)} \cdot f\left(a_{(1)}^{t} \cdot m\right)
$$

where $a \mapsto a^{t}$ is the left $\mathrm{F}$-linear automorphism of $\mathrm{F}_{W}$ given by $w \mapsto w^{-1}$.

Remark 3.15. Note that in the expression $\Delta(a)=\sum_{(a)} a_{(1)} \otimes a_{(2)}$, it may be that a particular summand $a_{(1)} \otimes a_{(2)}$ is NOT in $\mathrm{F}_{W}^{\prime}$. An example is $\Delta\left(A_{i}\right)=A_{i} \otimes 1+s_{i} \otimes A_{i}$, where neither of the summands $A_{i} \otimes 1$ and $s_{i} \otimes A_{i}$, is in $\mathrm{F}_{W}^{\prime}$. For such individual summands the componentwise action is ill-defined; see the following paragraph. Nevertheless if the ill-defined componentwise action is applied to each summand of an element of $\mathrm{F}_{W}^{\prime}$ and the results are added, the end result yields a well-defined action.

The naive componentwise product structure, which is well-defined on $\mathrm{F}_{W}^{\prime} \subset \mathrm{F}_{W} \otimes_{\mathrm{F}} \mathrm{F}_{W}$, does not extend to $\mathrm{F}_{W} \otimes_{\mathrm{F}} \mathrm{F}_{W}$. Take $q \in \mathrm{F}$ and $u, v \in W$. We recall that $q \operatorname{id} \otimes w-\mathrm{id} \otimes q w=0$. 
Suppose the componentwise product was well-defined on $\mathrm{F}_{W} \otimes_{\mathrm{F}} \mathrm{F}_{W}$. Then we would have

$$
\begin{aligned}
0 & =(u \otimes \mathrm{id}) \cdot(q \mathrm{id} \otimes w-\mathrm{id} \otimes q w) \\
& \stackrel{?}{=} u q \mathrm{id} \otimes w-u \otimes \mathrm{id} q w \\
& =(u \cdot q) u \otimes w-q u \otimes w \\
& =(-q+u \cdot q) u \otimes w
\end{aligned}
$$

But $u \cdot q \neq q$ in general, giving a contradiction.

The following result is proved in Appendix A.

\section{Proposition 3.16.}

1. The map $\Delta: \mathrm{F}_{W} \rightarrow \mathrm{F}_{W} \otimes_{\mathrm{F}} \mathrm{F}_{W}$ induces the unique left $S$-module homomorphism $\Delta: \mathbb{A} \rightarrow \mathbb{A} \otimes_{S} \mathbb{A}$ such that

$$
\begin{aligned}
\Delta\left(A_{i}\right) & =A_{i} \otimes 1+s_{i} \otimes A_{i} & & \\
& =1 \otimes A_{i}+A_{i} \otimes s_{i} & & \text { for all } i \in I \\
\Delta(a b) & =\Delta(a) \Delta(b) & & \text { for all } a, b \in \mathbb{A},
\end{aligned}
$$

where the product on $\operatorname{Im}(\Delta)$ is defined by the componentwise product (3.32).

2. Let $M$ and $N$ be left $\mathbb{A}$-modules. Then there is an action of $\mathbb{A}$ on $M \otimes_{S} N$ defined by

$$
\begin{aligned}
A_{i} \cdot(m \otimes n) & =\left(A_{i} \cdot m\right) \otimes n+\left(s_{i} \cdot m\right) \otimes\left(A_{i} \cdot n\right) \\
& =m \otimes\left(A_{i} \cdot n\right)+\left(A_{i} \cdot m\right) \otimes\left(s_{i} \cdot n\right) \\
q \cdot(m \otimes n) & =q m \otimes n
\end{aligned}
$$

for $i \in i$ and $q \in S$.

3. There is a left $\mathbb{A}$-action on $\operatorname{Hom}_{S}(M, N)$ given by

$$
\begin{aligned}
(q \cdot f)(m) & =q \cdot f(m) \\
\left(A_{i} \cdot f\right)(m) & =A_{i} \cdot f\left(s_{i} \cdot m\right)+f\left(A_{i} \cdot m\right) \\
& =s_{i} \cdot f\left(A_{i} \cdot m\right)+A_{i} \cdot f(m) \\
(w \cdot f)(m) & =w \cdot f\left(w^{-1} \cdot m\right) .
\end{aligned}
$$

for $q \in S, f \in \operatorname{Hom}_{S}(M, N), m \in M, i \in I$, and $w \in W$.

The Theorem allows us to perform computations such as

$$
\begin{aligned}
\Delta\left(A_{i} A_{j}\right) & =\Delta\left(A_{i}\right) \Delta\left(A_{j}\right) \\
& =\left(A_{i} \otimes 1+s_{i} \otimes A_{i}\right)\left(A_{j} \otimes 1+s_{j} \otimes A_{j}\right) \\
& =A_{i} A_{j} \otimes 1+A_{i} s_{j} \otimes A_{j}+s_{i} A_{j} \otimes A_{i}+s_{i} s_{j} \otimes A_{i} A_{j} .
\end{aligned}
$$




\section{Lemma 3.17.}

$$
\Delta\left(A_{w}\right) \in w \otimes A_{w}+\bigoplus_{u<w} \mathbb{A} \otimes A_{u}
$$

Exercise 3.18. Prove Lemma 3.17.

\subsection{Duality and the GKM ring}

Let $\mathrm{F}_{W}^{*}=\operatorname{Hom}_{\mathrm{F}-\mathrm{Mod}}\left(\mathrm{F}_{W}, \mathrm{~F}\right)$ be the F-vector space of left F-linear maps $\mathrm{F}_{W} \rightarrow \mathrm{F}$. It has two left actions of $\mathrm{F}_{W}$, one given by Proposition 3.16 and denoted by $\cdot$, and a commuting $\mathrm{F}_{W}$-action

$$
(b \bullet f)(a)=f(a b)
$$

for all $a, b \in \mathrm{F}_{W}$ and $f \in \mathrm{F}_{W}^{*}$. Define the left F-bilinear evaluation pairing

$$
\begin{aligned}
\mathrm{F}_{W}^{*} \times \mathrm{F}_{W} & \rightarrow \mathrm{F} \\
\langle f, a\rangle & =f(a) .
\end{aligned}
$$

Define the GKM ring $\Lambda \subset \mathrm{F}_{W}^{*}$ by

$$
\begin{aligned}
\hat{\Lambda} & =\left\{f \in \mathrm{F}_{W}^{*} \mid f(\mathbb{A}) \subset S\right\} \\
& =\left\{f \in \mathrm{F}_{W}^{*} \mid f\left(A_{w}\right) \in S \text { for all } w \in W\right\} \\
\Lambda & =\left\{f \in \hat{\Lambda} \mid f\left(A_{w}\right) \neq 0 \text { for finitely many } w \in W\right\} .
\end{aligned}
$$

The $\cdot$ action of $\mathrm{F}_{W}$ on $\mathrm{F}_{W}^{*}$ restricts to a left $S$-action on $\Lambda$ and $\hat{\Lambda}$ :

$$
(s f)(a)=s f(a)=f(s a) \quad \text { for all } f \in \hat{\Lambda}, s \in S, a \in \mathrm{F}_{W} .
$$

By Lemma 3.6 any element of $\Lambda$, being left $S$-linear, is completely determined by its values on the basis $\left\{A_{w} \mid w \in W\right\}$. The following is immediate.

Proposition 3.19. $\Lambda$ is a free $S$-module. It has a unique $S$-basis $\left\{\xi^{v} \mid v \in W\right\}$ defined by

$$
\left\langle\xi^{v}, A_{w}\right\rangle=\delta_{v w} .
$$

Similarly we have the direct product

$$
\hat{\Lambda}=\prod_{w \in W} S \xi^{v} .
$$

By definition, the pairing (3.46) restricts to a pairing

$$
\mathbb{A} \times \Lambda \rightarrow S
$$


Lemma 3.20. Both actions $\cdot$ and $\bullet$ of $\mathrm{F}_{W}$ on $\mathrm{F}_{W}^{*}$ defined by (3.45) restrict to an action of $\mathbb{A}$ on $\hat{\Lambda}$ and $\Lambda$.

Proof. Let $f \in \hat{\Lambda}$ and $w \in W$. By definition it is easy to see that $S \cdot \hat{\Lambda} \subset \hat{\Lambda}$. We have

$$
\left(A_{i} \cdot f\right)\left(A_{w}\right)=s_{i} \cdot f\left(A_{i} A_{w}\right)+A_{i} \cdot f\left(A_{w}\right) \in S
$$

by Proposition 3.16, (3.11) and Lemma 3.5. Since $\mathbb{A}$ is generated by $S$ and the $A_{i}$, it follows that $\mathbb{A} \cdot \hat{\Lambda} \subset \hat{\Lambda}$.

To show that $\mathbb{A} \bullet \hat{\Lambda} \subset \hat{\Lambda}$, it suffices to check that $(a \bullet f)\left(A_{w}\right) \in S$ for $a=A_{i}$ for $i \in I$ and $a=\lambda \in X \subset S$. Using (3.11) we have

$$
\begin{aligned}
\left(A_{i} \bullet f\right)\left(A_{w}\right) & =f\left(A_{w} A_{i}\right) \\
& =\chi\left(w s_{i}>w\right) f\left(A_{w s_{i}}\right) \in S
\end{aligned}
$$

since $f \in \hat{\Lambda}$. For $\lambda \in X$, by Proposition 3.7 we have

$$
\begin{aligned}
(\lambda \bullet f)\left(A_{w}\right) & =f\left(A_{w} \lambda\right) \\
& =(w \cdot \lambda) f\left(A_{w}\right)+\sum_{\substack{\alpha \in \Phi_{\mathrm{r}}^{+} \\
w s_{\alpha} \ltimes w}}\left\langle\alpha^{\vee}, \lambda\right\rangle f\left(A_{w s_{\alpha}}\right)
\end{aligned}
$$

which is in $S$ as required.

Lemma 3.21. For every $f \in \hat{\Lambda}$ we have

$$
f\left(s_{\alpha} w\right)-f(w) \in \alpha S \quad \text { for all } w \in W \text { and } \alpha \in \Phi_{\mathrm{re}} .
$$

We call (3.53) the GKM condition since it is an instance (for $\tilde{\mathrm{Fl}}$ ) of a general method due to [57] of constructing equivariant cohomology for suitable spaces. For Fl this is due to Kostant and Kumar [75].

Proof. Let $\beta=w^{-1} \cdot \alpha$. Then $w^{-1} s_{\alpha} w=s_{\beta}$ or $w s_{\beta}=s_{\alpha} w$. We have

$$
\begin{aligned}
\left(A_{\beta} \bullet f\right)(w) & =f\left(w A_{\beta}\right) \\
& =f\left(w \beta^{-1}\left(1-s_{\beta}\right)\right) \\
& =f\left(\alpha^{-1} w\left(1-s_{\beta}\right)\right) \\
& =\alpha^{-1}\left(f(w)-f\left(w s_{\beta}\right)\right) \\
& =\alpha^{-1}\left(f(w)-f\left(s_{\alpha} w\right)\right) .
\end{aligned}
$$

Since $A_{\beta} \bullet f \in \hat{\Lambda}$ by Lemma 3.20 and $w \in \mathbb{A}$ by (3.22), the right hand side of (3.54) is in $S$ as required. 
Lemma 3.22. For all $v \in W$ and $i \in I$

$$
\begin{aligned}
A_{i} \cdot \xi^{v} & =\chi\left(s_{i} v<v\right) \xi^{s_{i} v} \\
A_{i} \bullet \xi^{v} & =\chi\left(v s_{i}<v\right) \xi^{v s_{i}} .
\end{aligned}
$$

Exercise 3.23. Prove Lemma 3.22.

Lemma 3.24.

$$
\xi^{v}(w)=d_{v w} \quad \text { for } v, w \in W .
$$

Proof. By (3.23) we have

$$
\begin{aligned}
\left\langle\xi^{v}, w\right\rangle & =\left\langle\xi^{v}, \sum_{u \in W} d_{u w} A_{u}\right\rangle \\
& =\sum_{u} d_{u w}\left\langle\xi^{v}, A_{u}\right\rangle \\
& =\sum_{u} d_{u w} \delta_{u v} \\
& =d_{v w} .
\end{aligned}
$$

Using the corresponding properties of $d_{v w}$ we have the following.

\section{Proposition 3.25.}

1. $\xi^{v}(w) \in S$ is either 0 or a homogeneous polynomial of degree $\ell(v)$.

2.

$$
\xi^{v}(w)=0 \quad \text { unless } v \leq w .
$$

3.

$$
\begin{aligned}
& \xi^{v}(\mathrm{id})=\delta_{v, \mathrm{id}} \\
& \xi^{v}(w)=\xi^{v}\left(w s_{i}\right)+\chi\left(v s_{i}<v\right)\left(w \cdot \alpha_{i}\right) \xi^{v s_{i}}(w) \quad \text { if } w s_{i}<w .
\end{aligned}
$$

4. $(-1)^{\ell(v)} \xi^{v}(w) \in \mathbb{Z}_{\geq 0}\left[\alpha_{i} \mid i \in I\right]$.

5.

$$
(-1)^{\ell(v)} \xi^{v}(v)=\prod_{\substack{\alpha \in \Phi_{\mathrm{re}}^{+} \\ s_{\alpha} v<v}} \alpha .
$$


6. [2] [17]

$$
(-1)^{\ell(v)} \xi^{v}(w)=\sum_{\substack{b \in\{0,1\}^{\ell} \\ \prod_{b_{j}=1} A_{i_{j}}=A_{v}}}\left(\prod_{j=1}^{\ell} \alpha_{i_{j}}^{b_{j}} s_{i_{j}}\right) \cdot 1 .
$$

For $f \in \mathrm{F}_{W}^{*}$, define $\operatorname{Supp}(f)$ and $\Omega(f)$ by

$$
\begin{aligned}
& \operatorname{Supp}(f)=\{w \in W \mid f(w) \neq 0\} \\
& \quad \Omega(f)=\text { largest subset of } W \backslash \operatorname{Supp}(f) \text { such that } \\
& \quad v \in \Omega(f) \text { and } u \leq v \text { implies } u \in \Omega(f) \text { for all } u, v \in W .
\end{aligned}
$$

Proposition 3.26. Let $f \in \mathrm{F}_{W}^{*}$. Then $f \in \hat{\Lambda}$ if and only if $f$ satisfies the GKM condition (3.53).

Proof. The forward direction holds by Lemma 3.21. Conversely let $f \in \mathrm{F}_{W}^{*}$ satisfy the GKM condition (3.53). The proof proceeds by induction on $\Omega(f)$. Let $x \in W$ be minimal such that $x \notin \Omega(f)$. Let $\beta \in \Phi_{\mathrm{re}}^{+}$such that $s_{\beta} x<x$. Then $s_{\beta} x \in \Omega(f)$ and $f\left(s_{\beta} x\right)=0$. By (3.53) $f(x) \in \beta S$. As this holds for all such $\beta$ we have $f(x) \in \xi^{x}(x) S$ by Lemma 2.5 and Proposition 3.25. The function $g(w)=f(w)-\left(f(x) / \xi^{x}(x)\right) \xi^{x}(w)$ satisfies the GKM condition because both $f$ and $\xi^{x}$ do. However $\Omega(g) \supsetneq \Omega(f)$. By induction $g \in \hat{\Lambda}$ and therefore $f \in \hat{\Lambda}$.

Remark 3.27. The proof of Proposition 3.26 gives an effective algorithm to expand elements of $\Lambda$ into Schubert classes.

Let $\lambda \in X \subset S$. Define $c^{\lambda} \in \mathrm{F}_{W}^{*}$ by

$$
c^{\lambda}(a)=a \cdot \lambda \quad \text { for all } a \in \mathrm{F}_{W} .
$$

Lemma 3.28. We have

$$
c^{\lambda}=\lambda \xi^{\mathrm{id}}+\sum_{i \in I}\left\langle\alpha_{i}^{\vee}, \lambda\right\rangle \xi^{s_{i}} \in \Lambda .
$$

Proof. Let $\alpha \in \Phi_{\text {re }}$ and $w \in W$. We have $c^{\lambda}\left(s_{\alpha} w\right)-c^{\lambda}(w)=s_{\alpha} w \cdot \lambda-w \cdot \lambda=-\left\langle\alpha^{\vee}, w \cdot \lambda\right\rangle \alpha \in$ $\alpha S$. By Proposition 3.26 we have $c^{\lambda} \in \hat{\Lambda}$. The expansion (3.66) may be deduced from (3.16).

Exercise 3.29. For $w \in S_{n}$, let $\mathfrak{S}_{w}(x ; y)$ be the double Schubert polynomial of Lascoux and Schützenberger [102] defined by $\mathfrak{S}_{w_{0}}(x ; y)=\prod_{i=1}^{n-1} \prod_{j=1}^{n-i}\left(x_{i}-y_{j}\right)$ where $w_{0} \in S_{n}$ is the longest element (the reversing permutation), and if $w s_{i}<w, \mathfrak{S}_{w s_{i}}(x ; y)=\partial_{i}^{x} \mathfrak{S}_{w}(x ; y)$, where $\partial_{i}^{x}=\left(x_{i}-x_{i+1}\right)^{-1}\left(1-s_{i}^{x}\right)$ where $s_{i}^{x}$ acts on the $x$ variables. Find the precise relationship between $\xi^{v}(w)$ for the type $A_{n-1}$ root datum and the specializations $\mathfrak{S}_{v}(x ; w x)$ where $\mathfrak{S}_{v}(x ; w x)$ means $\mathfrak{S}_{v}(x ; y)$ with the $y$ variables replaced by the permuted $x$ variables $w x$. 


\subsection{Multiplication in $\Lambda$ and coproduct in $\mathbb{A}$}

Let $\operatorname{Fun}(W, \mathrm{~F})$ be the set of functions $W \rightarrow \mathrm{F}$. There is a bijection $\mathrm{F}_{W}^{*} \rightarrow \operatorname{Fun}(W, \mathrm{~F})$ given by restriction to $W$. We shall use this identification without further mention. Then $\mathrm{F}_{W}^{*}$ is a commutative F-algebra using the usual left F-module structure and the pointwise product on $\operatorname{Fun}(W, \mathrm{~F})$ :

$$
(f g)(w)=f(w) g(w) \quad \text { for } f, g \in \mathrm{F}_{W}^{*} \text { and } w \in W .
$$

The above formula is incorrect if $w$ is replaced by a general element of $\mathrm{F}_{W}^{*}$; the definition of $\mathrm{F}_{W}^{*}$ says to first apply linearity in the argument.

Lemma 3.30. $\hat{\Lambda}$ is a commutative S-algebra.

For $u, v, w \in W$ define the equivariant Schubert structure constants $p_{u v}^{w} \in S$ by

$$
\xi^{u} \xi^{v}=\sum_{w \in W} p_{u v}^{w} \xi^{w}
$$

\section{Proposition 3.31.}

1. $p_{u v}^{w}$ is 0 or a homogeneous polynomial of degree $\ell(u)+\ell(v)-\ell(w)$.

2. $p_{u v}^{w}=0$ unless $u \leq w$ and $v \leq w$.

This implies that the expansion (3.68) is finite, so that $\Lambda$ is a commutative $S$-algebra under the pointwise product.

Exercise 3.32. Prove Lemma 3.30 and Proposition 3.31 .

The following Theorem requires work. See [59, 77]; the positivity property (aside from the obvious sign) is called Graham positivity.

Theorem 3.33. $(-1)^{\ell(u)+\ell(v)-\ell(w)} p_{u v}^{w} \in \mathbb{Z}_{\geq 0}\left[\alpha_{i} \mid i \in I\right]$.

Problem 3.34. Find an explicit manifestly Graham-positive formula for $p_{u v}^{w}$.

The $p_{u v}^{w}$ have a geometric description as equivariant intersection numbers of Schubert varieties in $\tilde{F}$ l. Littlewood-Richardson numbers are a very special case. The complete answer is unknown even for $G=G L_{n}$ and for the case $\ell(w)=\ell(u)+\ell(v)$ when $p_{u v}^{w}$ are integers; this is the case of multiplying Schubert polynomials.

\section{Proposition 3.35.}

$$
c^{\lambda} \xi^{v}=(v \cdot \lambda) \xi^{v}+\sum_{\substack{\alpha \in \Phi_{\mathrm{re}}^{+} \\ v \ll v s_{\alpha}}}\left\langle\alpha^{\vee}, \lambda\right\rangle \xi^{v s_{\alpha}} .
$$


Corollary 3.36. For all $v \in W$ and $i \in I$,

$$
\xi^{s_{i}} \xi^{v}=\left(v \cdot \omega_{i}-\omega_{i}\right) \xi^{v}+\sum_{\substack{\alpha \in \Phi_{\text {re }}^{+} \\ v \ll v s_{\alpha}}}\left\langle\alpha^{\vee}, \omega_{i}\right\rangle \xi^{v s_{\alpha}} .
$$

Lemma 3.37.

$$
p_{v w}^{w}=\xi^{v}(w)
$$

Exercise 3.38. Prove Proposition 3.35, Corollary 3.36, and Lemma 3.37.

The product in $\Lambda$ can be recovered in $\mathbb{A}$ by duality. Recall $\Delta$ from (3.31). Define the left $S$-bilinear pairing

$$
\begin{aligned}
\langle\cdot, \cdot\rangle:\left(\Lambda \otimes_{S} \Lambda\right) \times \operatorname{Im}(\Delta) & \rightarrow S \\
\langle f \otimes g, \Delta(a)\rangle & =\sum_{a} f\left(a_{(1)}\right) g\left(a_{(2)}\right)
\end{aligned}
$$

Lemma 3.39. For $f, g \in \Lambda$ and $a \in \mathbb{A}$, we have

$$
\langle f g, a\rangle=\langle f \otimes g, \Delta(a)\rangle .
$$

Proof. We give the proof over F. By linearity we may assume $a=w \in W$. We have

$$
\langle f \otimes g, \Delta(w)\rangle=\langle f \otimes g, w \otimes w\rangle=f(w) g(w)=\langle f g, w\rangle .
$$

Applying (3.74) we see that

$$
\begin{aligned}
p_{u v}^{w} & =\left\langle\xi^{u} \xi^{v}, A_{w}\right\rangle \\
& =\left\langle\xi^{u} \otimes \xi^{v}, \Delta\left(A_{w}\right)\right\rangle .
\end{aligned}
$$

Therefore $p_{u v}^{w}$ is the coefficient of $A_{u} \otimes A_{v}$ in $\Delta\left(A_{w}\right)$. Let $w=s_{i_{1}} \cdots s_{i_{\ell}}$ be a reduced decomposition. By Proposition 3.16, $\Delta(w)$ can be computed as the componentwise product of $\Delta\left(A_{i_{1}}\right) \cdots \Delta\left(A_{i_{\ell}}\right)$.

\subsection{Forgetting equivariance}

Recall that $X \subset S$ is the subspace of linear polynomials. Consider the ring homomorphism $\epsilon_{0}^{S}: S \rightarrow \mathbb{Z}$ defined by the property $\epsilon_{0}^{S}(1)=1$ and $\epsilon_{0}^{S}(X)=0$. Define the ring homomorphism $\epsilon_{0}^{\Lambda}: \Lambda \rightarrow \Lambda_{0}:=\mathbb{Z} \otimes_{S} \Lambda$. It may be computed as follows. Write $f \in \Lambda$ as $f=\sum_{v \in W} f_{v} \xi^{v}$ for some $f_{v} \in S$. Then $\epsilon_{0}^{\Lambda}(f)=\sum_{v \in W} \epsilon_{0}^{S}\left(f_{v}\right) \xi_{0}^{v}$ where $\xi_{0}^{v}=\epsilon_{0}^{\Lambda}\left(\xi^{v}\right) \in \Lambda_{0}$. 
Proposition 3.40. $\left\{\xi_{0}^{v} \mid v \in W\right\}$ is a $\mathbb{Z}$-basis of $\Lambda_{0}$. This basis has structure constants given by the degree zero structure constants of $\Lambda$ :

$$
\begin{aligned}
\xi_{0}^{u} \xi_{0}^{v} & =\sum_{w \in W} \phi_{0}\left(p_{u v}^{w}\right) \xi_{0}^{w} \\
& =\sum_{\substack{w \in W \\
\ell(w)=\ell(u)+\ell(v)}} p_{u v}^{w} \xi_{0}^{w}
\end{aligned}
$$

The following is due to Lam [79]; see also Chapter 3 Section 8.1. Define $\epsilon_{0}^{\mathbb{A}}: \mathbb{A} \rightarrow \mathbb{Z} \otimes_{S} \mathbb{A}$ by $\epsilon_{0}^{\mathbb{A}}(a)=1 \otimes a$. By abuse of notation we write $A_{w}$ for its image under $\epsilon_{0}^{\mathbb{A}}$. Then for $a_{w} \in S$ we have

$$
\epsilon_{0}^{\mathbb{A}}\left(\sum_{w \in W} a_{w} A_{w}\right)=\sum_{w \in W} \epsilon_{0}^{S}\left(a_{w}\right) A_{w}
$$

There is a ring isomorphism $\mathbb{Z} \otimes_{S} \mathbb{A} \cong \mathbb{A}_{0}$ where $\mathbb{A}_{0}$ is the nilCoxeter algebra. Using this identification we may view $\epsilon_{0}^{\mathbb{A}}: \mathbb{A} \rightarrow \mathbb{A}_{0}$.

Example 3.41. For the root datum of type $A_{1}, W=\{\mathrm{id}, s\}, \Phi_{\mathrm{re}}^{+}=\{\alpha\}$, $\xi^{\text {id }}(\mathrm{id})=\xi^{\mathrm{id}}(s)=$ $1, \xi^{s}(\mathrm{id})=0$ and $\xi^{s}(s)=-\alpha$. Then $\xi^{\text {id }}$ is the identity in $\Lambda$ and $\xi^{s} \xi^{s}=-\alpha \xi^{s}$. In $\Lambda_{0}, \xi_{0}^{\text {id }}$ is the identity and $\left(\xi_{0}^{s}\right)^{2}=0$.

\subsection{Parabolic case}

Let $J \subset I$ be a fixed subset. Let $W_{J} \subset W$ be the subgroup generated by $s_{i}$ for $i \in J$. Let $W^{J}$ denote the set of minimum length coset representatives in $W / W_{J}$. Define

$$
\Lambda^{J}=\left\{f \in \Lambda \mid f(w u)=f(w) \text { for all } w \in W, u \in W_{J}\right\}
$$

Proposition 3.42. $\Lambda^{J}$ is an $S$-subalgebra of $\Lambda$ with basis $\left\{\xi^{v} \mid v \in W^{J}\right\}$.

Remark 3.43. There are polynomial or symmetric function representatives for many cases of cohomological Schubert classes. Nonequivariant:

1. Grassmannian $\operatorname{Gr}(r, n)$. Schur functions.

2. Lagrangian Grassmannian $L G(n, 2 n)$. Schur $Q$-functions. [134].

3. Orthogonal Grassmannians $O G(n, 2 n+1)$ and $O G(n+1,2 n+2)$ : Schur $P$-functions. [135].

4. Fl for $S L_{n}$ : Lascoux-Schützenberger Schubert polynomials [109].

5. Fl for $S p_{2 n}, S O_{2 n+1}, S O_{2 n}$ : Billey-Haiman Schubert polynomials [19]. 
6. $\operatorname{Gr}_{S L_{k+1}}$ : dual $k$-Schur or affine Schur functions [96] [97] [78] [79].

7. $\operatorname{Gr}_{S p_{2 n}}:[84]$.

8. $\operatorname{Gr}_{S O_{n}}:[132]$.

Equivariant:

1. Fl for $S L_{n}$ : Double Schubert polynomials [102]

2. Fl in classical type: BCD double Schubert polynomials [65].

\subsection{Geometric interpretations}

Fix a root datum. Let $T \subset B \subset G$ be a maximal torus contained in a Borel subgroup $B$ in the Kac-Moody group $G$ [77]. The Kac-Moody flag ind-variety $\mathrm{Fl}=G / B$ [77] is paved by cells $B \dot{w} B / B \cong \mathbb{C}^{\ell(w)}$ whose closures $X_{w}$ define equivariant fundamental homology classes $\left[X_{w}\right]_{T} \in H_{T}(\mathrm{Fl})$. Finally, the homology $H_{T}(\mathrm{Fl})$ has a noncommutative ring structure. Let $G$ act diagonally on $\mathrm{Fl} \times \mathrm{Fl}$. There are isomorphisms

$$
H_{G}(\mathrm{Fl} \times \mathrm{Fl}) \cong H_{G \times(B \times B)}(G \times G) \cong H_{B \times B}(G) \cong H_{B}(\mathrm{Fl}) \cong H_{T}(\mathrm{Fl}) .
$$

The left hand side has a convolution product [55]. There are also isomorphisms

$$
H_{G}(\mathrm{Fl}) \cong H_{G \times B}(G) \cong H_{B}(G \backslash G) \cong H_{B}(\mathrm{pt}) \cong H_{T}(\mathrm{pt})
$$

Finally, $H_{G}(\mathrm{Fl} \times \mathrm{Fl})$ acts on $H_{G}(\mathrm{Fl})$ by convolution [55]. The following is [55, Prop. 12.8].

Theorem 3.44. 1. There is an injective ring homomorphism

$$
H_{T}(\mathrm{pt}) \cong H_{G}(\mathrm{Fl}) \rightarrow H_{G}(\mathrm{Fl} \times \mathrm{Fl})
$$

given by composing the isomorphism (3.79) with the embedding induced by the Gequivariant diagonal inclusion $\mathrm{Fl} \rightarrow \mathrm{Fl} \times \mathrm{Fl}$.

2. There is an injective ring homomorphism

$$
\begin{aligned}
& \mathbb{A}_{0} \rightarrow H_{G}(G / B \times G / B) \\
& A_{w} \mapsto\left[O_{w}\right]_{G} \quad \text { for } w \in W
\end{aligned}
$$

where $\left[O_{w}\right]_{G}$ is the $G$-equivariant fundamental class of the closure $O_{w}$ of the diagonal $G$-orbit of the point $(\dot{e} B / B, \dot{w} B / B)$.

3. The above maps define a ring and left $S$-module isomorphism

$$
\mathbb{A} \cong H_{G}(\mathrm{Fl} \times \mathrm{Fl}) .
$$


4. The following diagram commutes where the horizontal maps are action maps and the vertical maps are isomorphisms.

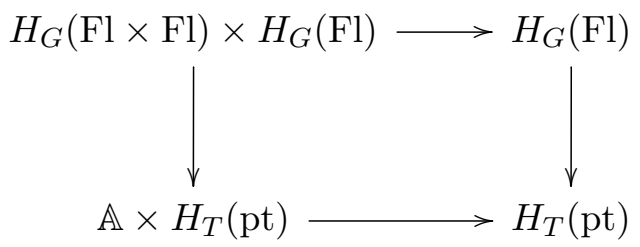

There is another kind of Kac-Moody flag variety $\tilde{\mathrm{F}}$ l with $T$-action called a thick flag scheme [68]. There is a bijection $\tilde{\mathrm{Fl}}{ }^{T} \cong W$ of the $T$-fixed point set of $\tilde{\mathrm{Fl}}$ with $W$. The thick flag scheme $\tilde{\mathrm{F}} \mathrm{l}$ is paved by finite-codimensional cells, each of which contains a unique $T$-fixed point. The closure of the cell containing $w$ is denoted $X^{w}$ and defines a class $\left[X^{w}\right]^{T} \in H^{T}(\tilde{\mathrm{F}})$ such that $\left\{\left[X_{w}\right]_{T} \mid w \in W\right\}$ and $\left\{\left[X^{w}\right]^{T} \mid w \in W\right\}$ are dual bases under an intersection pairing

$$
H_{T}(\mathrm{Fl}) \times H^{T}(\tilde{\mathrm{Fl}}) \rightarrow H^{T}(\mathrm{pt}) .
$$

For $w \in W$, let $i_{w}$ be the inclusion of a point into $\tilde{F} l$ whose image is the $w$-th $T$-fixed point. See [3] [75][77] for Fl and [68] [69] for F̂l.

Theorem 3.45. There is a commutative diagram

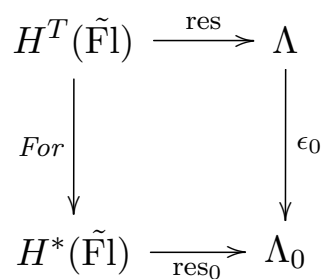

where For is the ring homomorphism that forgets equivariance, res is the $S=H^{T}(\mathrm{pt})$ algebra isomorphism that restricts a T-equivariant class to the set $\tilde{\mathrm{Fl}}^{T} \cong W$ of $T$-fixed points (and thus sends the T-equivariant class $\left[X^{v}\right]^{T}$ to $\xi^{v}$ ), and res $\mathrm{r}_{0}$ is a ring isomorphism sending the ordinary cohomology class $\left[X^{v}\right]$ to $\xi_{0}^{v}$. Moreover

$$
\xi^{v}(w)=i_{w}^{*}\left(\left[X^{v}\right]^{T}\right) .
$$

Proposition 3.46. The pairing (3.84) corresponds, using the isomorphisms (3.83) and (3.85), to the pairing (3.52).

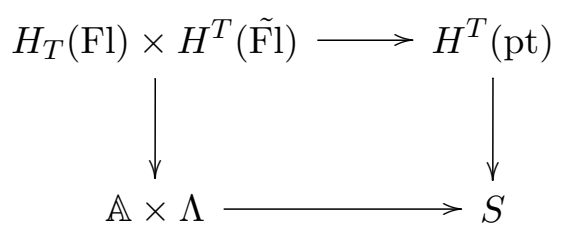


For $i \in I$ let $p_{i}: \tilde{\mathrm{F} l} \rightarrow \tilde{\mathrm{Fl}}^{i}$ be the projection of $\tilde{\mathrm{F} l}$ onto the thick partial flag scheme defined by the minimal parabolic subgroup corresponding to $i$.

Proposition 3.47. Under the isomorphism res of Theorem 3.45:

1. The first Chern class $c_{1}\left(\mathcal{L}_{\lambda}\right)$ of the T-equivariant line bundle on $\tilde{\mathrm{F}} \mathrm{l}$ of weight $\lambda$, maps to the function $c^{\lambda}$, and multiplication by $c_{1}\left(\mathcal{L}_{\lambda}\right)$ in $H^{T}(\tilde{\mathrm{F}})$ corresponds to the operation $f \mapsto \lambda \bullet f$ on $\Lambda$.

2. The push-pull operator $p_{i}^{*} p_{i *}$ on $H^{T}(\tilde{\mathrm{F}} \mathrm{l})$ corresponds to the operation $f \mapsto A_{i} \bullet f$ on $\Lambda$.

By the definitions (3.45) and (3.65), for $f \in \hat{\Lambda}, \lambda \in X$, and $w \in W$ we have

$$
(\lambda \bullet f)(w)=(w \cdot \lambda) f(w)=c^{\lambda}(w) f(w)=\left(c^{\lambda} f\right)(w) .
$$

Proposition 3.48. There is a commutative diagram

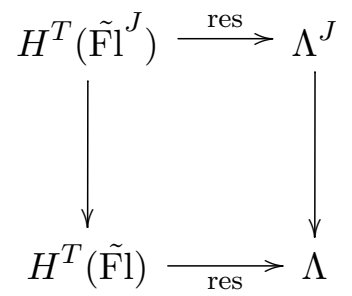

of S-algebra homomorphisms, where the horizontal maps are isomorphisms and the vertical maps are inclusions, where $\tilde{\mathrm{Fl}}^{J}$ is the thick partial flag scheme associated to the subset $J \subset I$. Under the top isomorphism, the Schubert basis of $H^{T}\left(\tilde{\mathrm{Fl}}{ }^{J}\right)$ maps to the elements $\xi^{v}$ for $v \in W^{J}$.

\section{Affine Grassmannian}

The affine Grassmannian $\operatorname{Gr}_{G}$ has the form $\mathrm{Gr}_{G}=G_{\text {af }} / P_{\text {af }}$ where $G_{\text {af }}$ is the Kac-Moody group of affine type associated with the semisimple algebraic group $G$ and $P_{\mathrm{af}}$ is the maximal parabolic subgroup obtained by "omitting the zero node". Therefore the previous sections apply and describe the Schubert calculus of $H^{T_{\mathrm{af}}}\left(\mathrm{Gr}_{G}\right)$ under the cup product, where $T_{\text {af }}$ is the maximal torus in $B_{\text {af }} \subset P_{\text {af }} \subset G_{\text {af }}$.

For our applications we consider $H^{T}\left(\mathrm{Gr}_{G}\right)$ where $T \subset B \subset G$ is a maximal torus in the semisimple algebraic group $G$ whose corresponding affine Kac-Moody group is $G_{\text {af }}$. Forgetting from $T_{\text {af }}$ to $T$ we retain the Schubert calculus of $H^{T}\left(\mathrm{Gr}_{G}\right)$. The algebraic model for this ring is denoted $\Lambda_{\mathrm{af}}^{\prime}$ and is studied in Section 4.2. It is due to Goresky, Kottwitz, and Macpherson. This ring also appears in Chapter 3 Section 7.2. 
However in this setting there are extra features. Instead of just the above ring, we get a dual Hopf algebra $H_{T}\left(\mathrm{Gr}_{G}\right)$ given by the equivariant homology of $\mathrm{Gr}_{G}$ under the Pontryagin product. The ring $H_{T}\left(\mathrm{Gr}_{G}\right)$ is very interesting in itself for several reasons.

One reason is due to Peterson [131] [88]: the Schubert structure constants of $H_{T}\left(\mathrm{Gr}_{G}\right)$ coincide with those of $Q H^{T}(G / B)$, the equivariant quantum cohomology of the flag manifolds $G / B$. Peterson realizes $H_{T}\left(\mathrm{Gr}_{G}\right)$ algebraically as a commutative subalgebra $\mathbb{B}$ of a variant of the affine nilHecke ring of Kostant and Kumar, which uses the affine Weyl group but polynomial functions on the Lie algebra of the finite torus, rather than the affine one. This variant is denoted $\mathbb{A}_{\mathrm{af}}$ in Chapter 3 Section 6.1 and $\mathbb{A}_{\mathrm{af}}^{\prime}$ in Section 4.4.

For the second reason we forget equivariance and consider $G=S L_{k+1}$. Then the ring $H_{*}\left(\mathrm{Gr}_{S L_{k+1}}\right)$ is Hopf-isomorphic to the subring $\mathbb{Z}\left[h_{1}, \ldots, h_{k}\right]$ of symmetric functions generated by the first $k$ complete symmetric functions, and the Schubert basis is given by the $k$-Schur functions of Lapointe, Lascoux, and Morse (at $t=1$ ). This basis is connected with Macdonald polynomials.

For $G$ of classical type, $H_{*}\left(\mathrm{Gr}_{G}\right)$ can be realized by symmetric functions and the Schubert bases give rise to new families of symmetric functions; see [84] [132].

\subsection{Affine Grassmannian as partial affine flags}

Consider a finite root datum with simple Lie group $G \supset B \supset T$, containing a Borel subgroup $B$ and maximal torus $T$. The affine Grassmannian is by definition $\mathrm{Gr}=\mathrm{Gr}_{G}=$ $G(\mathbb{C}((t))) / G(\mathbb{C}[[t]])$ where $\mathbb{C}[[t]]$ is the formal power series ring and $\mathbb{C}((t))$ is the formal Laurent series ring.

Consider the associated affine root datum with Kac-Moody group, Iwahori subgroup, and maximal torus $G_{\mathrm{af}} \supset B_{\mathrm{af}} \supset T_{\mathrm{af}}$. Let $\mathrm{Fl}_{\mathrm{af}}=G_{\mathrm{af}} / B_{\mathrm{af}}$ be the affine flag ind-variety where $B_{\text {af }}$ is the Iwahori (affine Borel) subgroup. Then (up to a central extension that gets modded out in the quotient) $G_{\mathrm{af}}=G(\mathbb{C}((t))), P_{\mathrm{af}}=G(\mathbb{C}[[t]])=P_{I}$ for the subset $I \subset I_{\mathrm{af}}$, and $\mathrm{Gr}=G_{\mathrm{af}} / P_{\mathrm{af}}=\mathrm{Fl}_{\mathrm{af}}^{I}$. Let $\tilde{\mathrm{F}} \mathrm{af}_{\mathrm{af}}$ be the thick affine flag scheme [68] and $\tilde{\mathrm{Gr}}=\tilde{\mathrm{F}} \mathrm{af}_{\mathrm{af}}^{I}$ the thick affine Grassmannian, the associated thick partial affine flag scheme.

The previous section describes isomorphisms

$$
\Lambda_{\mathrm{af}} \cong H^{T_{\mathrm{af}}}\left(\tilde{\mathrm{Gr}}_{G}\right) \quad \mathbb{A}_{\mathrm{af}} \cong H_{T_{\mathrm{af}}}\left(\mathrm{Gr}_{G}\right)
$$

Our applications require working with equivariance with respect to the "small torus" $T$ rather than the maximal torus $T_{\mathrm{af}}$ in $G_{\mathrm{af}}$. It is possible to modify the GKM ring $\Lambda_{\mathrm{af}}$ and the affine nilHecke ring $\mathbb{A}_{\mathrm{af}}$ to obtain rings $\Lambda_{\mathrm{af}}^{\prime}$ and $\mathbb{A}_{\mathrm{af}}^{\prime}$ such that

$$
\Lambda_{\mathrm{af}}^{\prime} \cong H^{T}\left(\tilde{\mathrm{Gr}}_{G}\right) \quad \mathbb{A}_{\mathrm{af}}^{\prime} \cong H_{T}\left(\mathrm{Gr}_{G}\right)
$$

The necessary modifications were obtained in [58] and [131] respectively.

Under the small torus equivariance, the homology ring $H_{T}\left(\mathrm{Gr}_{G}\right)$ becomes a commutative and cocommutative Hopf algebra over $S=H^{T}(p t)$, with dual Hopf algebra given by 
$H^{T}\left(\operatorname{Gr}_{G}\right)$. Our main interest lies in obtaining explicit computations involving this Hopf structure.

We write $W_{\mathrm{af}}^{0}$ instead of $W_{\mathrm{af}}^{I}$ and $\pi: X_{\mathrm{af}} \rightarrow X$ for the natural projection and also for the induced map $\pi: S_{\text {af }}=\operatorname{Sym}\left(X_{\mathrm{af}}\right) \rightarrow S=\operatorname{Sym}(X)$.

\subsection{Small torus version of $\Lambda_{\mathrm{af}}$}

Here we follow [58]. Consider the map $\pi^{*}: \Lambda_{\text {af }} \rightarrow \operatorname{Fun}\left(W_{\text {af }}, S\right)$ given by $\pi^{*}(f)=\pi \circ f$. We wish to characterize the image of $\pi^{*}$ and the image of its restriction to $\Lambda_{\mathrm{Gr}}$.

The usual GKM condition (3.53) holds for functions $f: W_{\mathrm{af}} \rightarrow S$ in the image of $\pi^{*}$, since it holds in $\Lambda_{\mathrm{af}}$. However there are more conditions.

Let $\Lambda_{\text {af }}^{\prime}$ be the set of functions $f \in \operatorname{Fun}\left(W_{\text {af }}, S\right)$ that satisfy (3.53) and the following small torus GKM conditions:

$$
\begin{aligned}
f\left(\left(1-t_{\alpha^{\vee}}\right)^{d} w\right) & \in \alpha^{d} S \\
f\left(\left(1-t_{\alpha}\right)^{d-1}\left(1-s_{\alpha}\right) w\right) & \in \alpha^{d} S
\end{aligned}
$$

for all $d \in \mathbb{Z}_{>0}, w \in W_{\text {af }}$, and $\alpha \in \Phi$.

Let $\Lambda_{\mathrm{Gr}}^{\prime}$ be the functions in $\Lambda_{\mathrm{af}}^{\prime}$ that are constant on cosets in $W_{\mathrm{af}} / W$.

Lemma 4.1. Suppose $f \in \operatorname{Fun}\left(W_{\mathrm{af}}, S\right)$ satisfies (4.1). Then for all $d \in \mathbb{Z}_{>0}, p \in \mathbb{Z}, \alpha \in \Phi$, and $w \in W_{\text {af }}$ we have

$$
f\left(\left(1-t_{\alpha^{\vee}}\right)^{d-1} w\right) \equiv f\left(\left(1-t_{\alpha^{\vee}}\right)^{d-1} t_{p \alpha^{\vee}} w\right) \quad \bmod \alpha^{d} S .
$$

Proof. By induction we may reduce to the case $p=1$, which is just (4.1).

Lemma 4.2. If $f \in \operatorname{Fun}\left(W_{\mathrm{af}}, S\right)$ satisfies (3.53) and (4.1) and is constant on cosets in $W_{\text {af }} / W$ then it also satisfies (4.2).

Proof. Let $\alpha \in \Phi, m \in \mathbb{Z}, d \in \mathbb{Z}_{>0}$, and $w=t_{\mu} u \in W_{\text {af }}$ with $\mu \in Q^{\vee}$ and $u \in W$. Let $y=\left(1-t_{\alpha} \vee\right)^{d-1}$. Using Lemma 4.1 we have

$$
\begin{aligned}
f\left(y\left(1-s_{\alpha}\right) t_{\mu} u\right) & =f\left(y t_{\mu} u\right)-f\left(y t_{s_{\alpha} \cdot \mu} s_{\alpha} u\right) \\
& =f\left(y t_{\mu}\right)-f\left(y t_{-\langle\mu, \alpha\rangle \alpha} t_{\mu}\right) \\
& \equiv f\left(y t_{\mu}\right)-f\left(y t_{\alpha \vee} t_{\mu}\right) \quad \bmod \alpha^{d} S \\
& =f\left(\left(1-t_{\alpha^{\vee}}\right)^{d} t_{\mu}\right) \in \alpha^{d} S .
\end{aligned}
$$

Define

$$
\bar{\xi}^{v}=\pi \circ \xi^{v} \quad \text { for } v \in W_{\text {af }} .
$$

The following Theorem is proved in Appendix B and is due to Goresky, Kottwitz, and Macpherson [58]. 
Theorem 4.3. $\quad$ 1. There is an S-algebra isomorphism

$$
\begin{aligned}
H^{T}\left(\tilde{\mathrm{F}} l_{\mathrm{af}}\right) & \rightarrow \Lambda_{\mathrm{af}}^{\prime} \cong \bigoplus_{w \in W_{\mathrm{af}}} S \bar{\xi}^{w} \\
{\left[X^{w}\right]^{T} } & \mapsto \bar{\xi}^{w} \quad \text { for } w \in W_{\mathrm{af}} .
\end{aligned}
$$

2. The isomorphism (4.5) restricts to an $S$-algebra isomorphism

$$
H^{T}(\tilde{\mathrm{Gr}}) \rightarrow \Lambda_{\mathrm{Gr}}^{\prime} \cong \bigoplus_{w \in W_{\mathrm{af}}^{0}} S\left(\bar{\xi}^{w}\right) .
$$

The existence of the following map is explained in Appendix C.

Proposition 4.4. The map $\varpi$ defined by

$$
\begin{aligned}
\varpi: \Lambda_{\mathrm{af}}^{\prime} & \rightarrow \Lambda_{\mathrm{Gr}}^{\prime} \\
f & \mapsto\left(t_{\mu} u \mapsto f\left(t_{\mu}\right)\right) \quad \text { for } \mu \in Q^{\vee} \text { and } u \in W
\end{aligned}
$$

is an $S$-algebra homomorphism which is the identity when restricted to $\Lambda_{\mathrm{Gr}}^{\prime} \subset \Lambda_{\mathrm{af}}^{\prime}$.

Proof of Prop. 4.4. All properties are clear except $\operatorname{Im}(\varpi) \subset \Lambda_{\mathrm{Gr}}^{\prime}$. Let $f \in \Lambda_{\mathrm{af}}^{\prime}$ and $g=$ $\varpi(f)$. By Lemma 4.2 it suffices to verify (4.1) and (3.53). For the former, let $d \in \mathbb{Z}_{>0}$ and $\alpha \in \Phi$. Let $w=t_{\mu} u \in W_{\text {af }}$ with $\mu \in Q^{\vee}$ and $u \in W$. We have

$$
g\left(\left(1-t_{\alpha^{\vee}}\right)^{d} w\right)=g\left(\left(1-t_{\alpha^{\vee}}\right)^{d} t_{\mu} u\right)=f\left(\left(1-t_{\alpha^{\vee}}\right)^{d} t_{\mu}\right) \in \alpha^{d} S
$$

by the definition of $\varpi$ and (4.1) for $f$. For (3.53), let $\alpha+m \delta \in \Phi_{\mathrm{re}}^{\text {af }}$ for $\alpha \in \Phi$ and $m \in \mathbb{Z}$. Let $w=t_{\mu} u$ for $\mu \in Q^{\vee}$ and $u \in W$. We have $s_{\alpha+m \delta} w=s_{\alpha} t_{m \alpha^{\vee}} t_{\mu} u=t_{-m \alpha} \vee t_{s_{\alpha} \cdot \mu} s_{\alpha} u=$ $t_{-(m+\langle\mu, \alpha\rangle) \alpha^{\vee}} t_{\mu} s_{\alpha} u$. Therefore

$$
g\left(s_{\alpha+m \delta} w\right)-g(w)=f\left(t_{-(m+\langle\mu, \alpha\rangle) \alpha^{\vee}} t_{\mu}\right)-f\left(t_{\mu}\right) \in \alpha S
$$

by Lemma 4.1 for $d=1$.

\subsection{Homology of the affine Grassmannian}

For $\mu \in Q^{\vee}$ define $i_{\mu}^{*} \in \operatorname{Hom}_{S}\left(\Lambda_{\mathrm{Gr}}^{\prime}, S\right)$ by

$$
i_{\mu}^{*}(f)=f\left(t_{\mu}\right) \quad \text { for } f \in \Lambda_{\mathrm{Gr}}^{\prime}
$$

In the notation of Theorem 3.45, $i_{\mu}^{*}=i_{t_{\mu}}^{*}$.

Lemma 4.5. $\mathrm{F} \otimes_{S} \operatorname{Hom}_{S}\left(\Lambda_{\mathrm{Gr}}^{\prime}, S\right)$ has F-basis $\left\{i_{\mu}^{*} \mid \mu \in Q^{\vee}\right\}$. 
Proof. Let $m_{\mu} \in W_{\text {af }}^{0}$ be defined by $m_{\mu} W=t_{\mu} W$ for $\mu \in Q^{\vee}$. By (3.61) the matrix $\xi^{v}(w)$ is triangular, so the same is true of its restriction $\bar{\xi}^{m_{\mu}}\left(t_{\nu}\right)$ for $\nu \in Q^{\vee}$. Moreover its diagonal entries are nonvanishing: since $\bar{\xi}^{m_{\mu}}$ is constant on the cosets of $W_{\text {af }} / W$ we have $\bar{\xi}^{m_{\mu}}\left(t_{\mu}\right)=\bar{\xi}^{m_{\mu}}\left(m_{\mu}\right) \neq 0$. The Lemma follows.

The following result is proved in Appendix C.

Proposition 4.6. $H_{T}(\mathrm{Gr})$ and $H^{T}(\tilde{\mathrm{Gr}})$ are dual Hopf algebras over $S$. There is an isomorphism $H_{T}(\mathrm{Gr}) \cong \operatorname{Hom}_{S}\left(\Lambda_{\mathrm{Gr}}^{\prime}, S\right)$ under which the product in $\operatorname{Hom}_{S}\left(\Lambda_{\mathrm{Gr}}^{\prime}, S\right)$ is defined over $\mathrm{F}$ by $\left(i_{\lambda}^{*}, i_{\mu}^{*}\right) \mapsto i_{\lambda+\mu}^{*}$. $\operatorname{Hom}_{S}\left(\Lambda_{\mathrm{Gr}}^{\prime}, S\right)$ and $\Lambda_{\mathrm{Gr}}^{\prime}$ have the structure of dual Hopf algebras over $S$ where the product in $\operatorname{Hom}_{S}\left(\Lambda_{\mathrm{Gr}}^{\prime}, S\right)$ is defined over $\mathrm{F}$ by $\left(i_{\lambda}^{*}, i_{\mu}^{*}\right) \mapsto i_{\lambda+\mu}^{*}$.

Define the $S$-basis $\left\{\xi_{w} \mid w \in W_{\mathrm{af}}^{0}\right\}$ of $\operatorname{Hom}_{S}\left(\Lambda_{\mathrm{Gr}}^{\prime}, S\right)$ by

$$
\left\langle\xi_{w}, \bar{\xi}^{v}\right\rangle=\delta_{w v} \quad \text { for } v \in W_{\mathrm{af}}^{0} .
$$

Remark 4.7. There is an isomorphism of Hopf $S$-algebras

$$
\begin{aligned}
H_{T}(\mathrm{Gr}) & \cong \operatorname{Hom}_{S}\left(\Lambda_{\mathrm{Gr}}^{\prime}, S\right) \\
{\left[X_{w}\right]_{T} } & \mapsto \xi_{w} \quad \text { for } w \in W_{\mathrm{af}}^{0}
\end{aligned}
$$

where $X_{w}=\overline{B_{\text {af }} \dot{w} P_{\text {af }} / P_{\text {af }}} \subset G_{\text {af }} / P_{\text {af }}=$ Gr is the Schubert variety and $\left[X_{w}\right]_{T}$ is the $T$ equivariant fundamental class. Moreover there is a perfect intersection pairing

$$
H_{T}(\mathrm{Gr}) \times H^{T}(\tilde{\mathrm{Gr}}) \rightarrow S
$$

which corresponds to the evaluation pairing

$$
\operatorname{Hom}_{S}\left(\Lambda_{\mathrm{Gr}}^{\prime}, S\right) \times \Lambda_{\mathrm{Gr}}^{\prime} \rightarrow S
$$

under the isomorphisms (4.10) and (4.3).

\subsection{Small torus affine nilHecke ring and Peterson subalgebra}

We denote by $\mathbb{A}_{\text {af }}$ the maximal torus affine nilHecke ring obtained from an untwisted affine root datum as in Section 3. The Peterson (small torus) affine nilHecke ring $\mathbb{A}_{\text {af }}^{\prime}$ (denoted $\mathbb{A}_{\mathrm{af}}$ in Chapter 3) is the subquotient of $\mathbb{A}_{\mathrm{af}}$ defined as follows.

Let $W_{\text {af }}$ be the affine Weyl group. It acts naturally on the affine weight lattice $X_{\text {af }}$ and preserves the level (2.41) of a weight. Let $X_{0} \subset X_{\text {af }}$ be the sublattice of level zero weights. By (2.36) we have $X_{\text {af }} \cong \mathbb{Z} \Lambda_{0} \oplus X_{0}$ where $\Lambda_{0}$ is the zero-th affine fundamental weight. There is a natural surjection $\pi: X_{0} \rightarrow X$ with kernel $\mathbb{Z} \delta$ where $\delta$ is the null root. Since the action of $W_{\text {af }}$ fixes $\delta$ there is an action of $W_{\text {af }}$ on $X$ called the level zero action; it is defined by (2.53). Let $\mathrm{F}_{\mathrm{af}}, \mathrm{F}_{0}$, and $\mathrm{F}$ be the fraction fields of the algebras $S_{\mathrm{af}}=\operatorname{Sym}\left(X_{\mathrm{af}}\right)$, $\left.S_{0}=\operatorname{Sym}_{(} X_{0}\right)$, and $S=\operatorname{Sym}(X)$ respectively. We have $S_{\text {af }}=S_{0}\left[\Lambda_{0}\right], S_{0} / \delta S_{0} \cong S$. 
In the definition of $\mathbb{A}_{\mathrm{af}}$ the elements $A_{i}$ lie in the twisted group algebra $\mathrm{F}_{\mathrm{af}} W_{\mathrm{af}}$, but since the variable $\Lambda_{0}$ does not appear in them, they are also elements of the subring $\mathrm{F}_{0} W_{\mathrm{af}}$. Let $\mathbb{A}_{\mathrm{af}}^{0}$ be the subring of $\mathrm{F}_{0} W_{\mathrm{af}}$ generated by $A_{i}$ for $i \in I_{\mathrm{af}}$ and $S_{0}$. Then $\mathbb{A}_{\text {af }}^{0}$ is naturally a subring of $\mathbb{A}_{\mathrm{af}}$ and adjoining the variable $\Lambda_{0}$ to $\mathbb{A}_{\mathrm{af}}^{0}$ yields $\mathbb{A}_{\mathrm{af}}: \mathbb{A}_{\mathrm{af}}=S_{\mathrm{af}} \otimes_{S_{0}} \mathbb{A}_{\mathrm{af}}^{0}$. Define

$$
\mathbb{A}_{\mathrm{af}}^{\prime}=S \otimes S_{0} \mathbb{A}_{\mathrm{af}}^{0}
$$

where $S_{0}$ acts on $S$ by the ring homomorphism $\pi: S_{0} \rightarrow S$. In other words, $\mathbb{A}_{\text {af }}^{\prime}$ is obtained from $\mathbb{A}_{\mathrm{af}}^{0}$ by setting the variable $\delta$ to zero. In particular, in $\mathbb{A}_{\mathrm{af}}^{\prime}$, since $\pi\left(\alpha_{0}\right)=\pi(\delta-\theta)=-\theta$, we have

$$
A_{0}=\left(1-s_{0}\right) /(-\theta)
$$

Alternatively one may define $\mathbb{A}_{\mathrm{af}}^{\prime}$ as the subring of $\mathrm{F} W_{\text {af }}$ (where $W_{\text {af }}$ acts on $\mathrm{F}$ by the level zero action) generated by $A_{i}$ for $i \in I_{\mathrm{af}}$ and $S$ with $A_{0}$ defined as in (4.15).

Any formula in $\mathbb{A}_{\mathrm{af}}$ that does not involve the variable $\Lambda_{0}$, automatically holds in $\mathbb{A}_{\text {af }}^{\prime}$ after applying $\pi$. By Lemma 3.6 (applied for an untwisted affine root datum, so that the Weyl group is $W_{\text {af }}$ and the polynomial ring is $S_{\text {af }}$ ) we have

$$
\mathbb{A}_{\mathrm{af}}^{\prime}=\bigoplus_{w \in W_{\mathrm{af}}} S A_{w}
$$

Evaluation yields left $S$-linear perfect pairings

$$
\begin{array}{r}
\mathbb{A}_{\mathrm{af}}^{\prime} \times \Lambda_{\mathrm{af}}^{\prime} \rightarrow S \\
\operatorname{Hom}_{S}\left(\Lambda_{\mathrm{Gr}}^{\prime}, S\right) \times \Lambda_{\mathrm{Gr}}^{\prime} \rightarrow S .
\end{array}
$$

Let $j: \operatorname{Hom}_{S}\left(\Lambda_{\mathrm{Gr}}^{\prime}, S\right) \rightarrow \mathbb{A}_{\mathrm{af}}^{\prime}$ be the left $S$-module homomorphism defined by

$$
\langle j(\xi), f\rangle=\langle\xi, \varpi(f)\rangle \quad \text { for } f \in \Lambda_{\mathrm{af}}^{\prime}, \xi \in \operatorname{Hom}_{S}\left(\Lambda_{\mathrm{Gr}}^{\prime}\right) .
$$

It is well-defined since we may vary $f$ over the basis $\left\{\bar{\xi}^{v} \mid v \in W_{\mathrm{af}}\right\}$ of $\Lambda_{\mathrm{af}}^{\prime}$. The map $j$ is injective, for $\varpi$ is onto by Proposition 4.4.

The following diagram relates the maps $\varpi, j$, and the pairings.

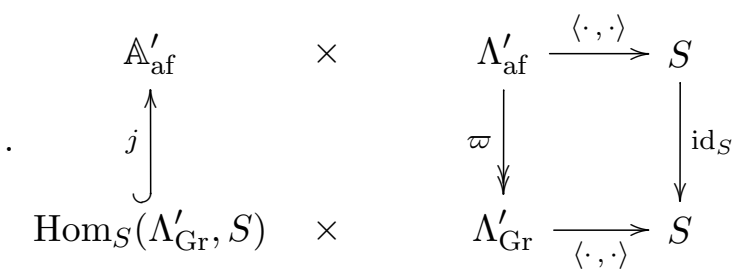

The Peterson subalgebra $\mathbb{P}$ is defined to be the centralizer of $S$ in $\mathbb{A}_{\mathrm{af}}^{\prime}$ :

$$
\mathbb{P}:=Z_{\mathbb{A}_{\mathrm{af}}^{\prime}}(S)
$$


Lemma 4.8. We have $\operatorname{Im}(j)=\bigoplus_{\mu \in Q^{\vee}} \mathrm{F} t_{\mu} \cap \mathbb{A}_{\mathrm{af}}^{\prime}=\mathbb{P}$.

Proof. For $\mu \in Q^{\vee}$, we have $\left\langle i_{\mu}^{*}, \varpi(f)\right\rangle=f\left(t_{\mu}\right)$ for all $f \in \mathbb{A}_{\text {af }}^{\prime}$, so that

$$
j\left(i_{\mu}^{*}\right)=t_{\mu} \in \mathbb{A}_{\mathrm{af}}^{\prime} \quad \text { for } \mu \in Q^{\vee} .
$$

The first equality holds by Lemma 4.5. For the second equality, $\bigoplus_{\mu \in Q^{\vee}} \mathrm{F} t_{\mu} \cap \mathbb{A}_{\text {af }}^{\prime} \subseteq \mathbb{P}$ holds because under the level zero action, $t_{\mu}$ acts on $X$ trivially for all $\mu \in Q^{\vee}$. For the other direction, let $a=\sum_{w \in W_{\mathrm{af}}} a_{w} w \in \mathbb{P}$ for $a_{w} \in \mathrm{F}$. Then for all $\lambda \in X$ we have

$$
0=\lambda a-a \lambda=\sum_{w \in W_{\mathrm{af}}} a_{w}(\lambda-w \cdot \lambda) w .
$$

Therefore for all $w \in W_{\text {af }}$ either $a_{w}=0$ or $w \lambda=\lambda$ for all $\lambda \in X$. Taking $\lambda$ to be $W$-regular, we see that the latter only holds for $w=t_{\mu}$ for some $\mu \in Q^{\vee}$.

The algebra $\mathbb{P}$ inherits a coproduct $\Delta: \mathbb{P} \rightarrow \mathbb{P} \otimes_{S} \mathbb{P}$ from the coproduct of $\mathbb{A}_{\text {af }}^{\prime}$. That $\Delta(\mathbb{P}) \subset \mathbb{P} \otimes_{S} \mathbb{P}$ follows from Lemma 4.8 and (3.31). We make $\mathbb{P}$ into a Hopf algebra over $S$ by defining the antipode $t_{\mu} \mapsto t_{-\mu}$ for $\mu \in Q^{\vee}$.

Theorem 4.9. [131] [79] The map $j: \operatorname{Hom}_{S}\left(\Lambda_{\mathrm{Gr}}^{\prime}, S\right) \rightarrow \mathbb{P}$ is a Hopf-isomorphism.

Proof. We have seen $j$ is injective, and $j$ is surjective by Lemma 4.8. It suffices to show $j$ is a bialgebra morphism, since the compatibility with antipodes follows as a consequence.

Since $j$ is $S$-linear, to check that $j$ is compatible with the Hopf-structure we check the product and coproduct structures on the $F$-basis $\left\{i_{\mu}^{*} \mid \mu \in Q^{\vee}\right\}$ of $\mathrm{F} \otimes_{S} \operatorname{Hom}_{S}\left(\Lambda_{\mathrm{Gr}}^{\prime}, S\right)$.

By Proposition 4.6 and (4.22), for $\lambda, \mu \in Q^{\vee}$ we have

$$
j\left(i_{\lambda}^{*} i_{\mu}^{*}\right)=j\left(i_{\lambda+\mu}^{*}\right)=t_{\lambda+\mu}=t_{\lambda} t_{\mu}=j\left(i_{\lambda}^{*}\right) j\left(i_{\mu}^{*}\right) .
$$

Thus $j$ is an algebra morphism.

To show $j$ is a coalgebra morphism, let $a \in \operatorname{Hom}_{S}\left(\Lambda_{\mathrm{Gr}}^{\prime}, S\right)$ and $f, g \in \Lambda_{\mathrm{Gr}}^{\prime}$. Then

$$
\begin{aligned}
\langle(j \otimes j) \circ \Delta(a), f \otimes g\rangle & =\sum_{a}\left\langle j\left(a_{(1)}\right) \otimes j\left(a_{(2)}\right), f \otimes g\right\rangle \\
& =\sum_{a}\left\langle j\left(a_{(1)}\right), f\right\rangle\left\langle j\left(a_{(2)}\right), g\right\rangle \\
& =\sum_{a}\left\langle a_{(1)}, \varpi(f)\right\rangle\left\langle a_{(2)}, \varpi(g)\right\rangle \\
& =\sum_{a}\left\langle a_{(1)} \otimes a_{(2)}, \varpi(f) \otimes \varpi(g)\right\rangle \\
& =\langle\Delta(a), \varpi(f) \otimes \varpi(g)\rangle \\
& =\langle a, \varpi(f) \varpi(g)\rangle \\
& =\langle a, \varpi(f g)\rangle \\
& =\langle j(a), f g\rangle \\
& =\langle\Delta(j(a)), f \otimes g\rangle .
\end{aligned}
$$




\subsection{The $j$-basis}

Peterson characterized the image $j_{w}$ of the Schubert basis element $\xi_{w}$ in $\mathbb{P}$ as follows.

Theorem 4.10. [131] [79] For each $w \in W_{\mathrm{af}}^{0}$, there is a unique element $j_{w} \in \mathbb{P}$ of the form

$$
j_{w}=A_{w}+\sum_{v \in W_{\mathrm{af}} \backslash W_{\mathrm{af}}^{0}} j_{w}^{v} A_{v}
$$

for some $j_{w}^{v} \in S$. Furthermore, $j_{w}=j\left(\xi_{w}\right)$ and $\mathbb{P}=\bigoplus_{w \in W_{\mathrm{af}}^{0}} S j_{w}$.

Proof. Since $\left\{\xi_{w} \mid w \in W_{\mathrm{af}}^{0}\right\}$ is an $S$-basis of $\operatorname{Hom}_{S}\left(\Lambda_{\mathrm{Gr}}^{\prime}, S\right)$, by Theorem 4.9, setting $j_{w}=j\left(\xi_{w}\right)$ we obtain an $S$-basis of $\mathbb{P}$. Let $v, w \in W_{\text {af }}^{0}$. We have

$$
\begin{aligned}
\left\langle j_{w}, \bar{\xi}^{v}\right\rangle & =\left\langle j\left(\xi_{w}\right), \bar{\xi}^{v}\right\rangle \\
& =\left\langle\xi_{w}, \varpi\left(\bar{\xi}^{v}\right)\right\rangle \\
& =\left\langle\xi_{w}, \bar{\xi}^{v}\right\rangle \\
& =\delta_{v w}
\end{aligned}
$$

by (4.19), Proposition 4.4, and (4.9). On the other hand $\left\langle j_{w}, \bar{\xi}^{v}\right\rangle$ is the coefficient of $A_{v}$ in $j_{w} \in \mathbb{P} \subset \mathbb{A}_{\text {af }}^{\prime}$. The form (4.23) follows. The element $j_{w} \in \mathbb{P}$ is unique because the set $\left\{A_{w} \mid w \in W_{\text {af }}^{0}\right\}$ is linearly independent.

For $w \in W_{\text {af }}^{0}$ let $t^{w}=t_{\lambda}$ where $\lambda \in Q^{\vee}$ is such that $t^{w} W=t_{\lambda} W$. Since $\left\{j_{v} \mid v \in W_{\text {af }}^{0}\right\}$ is an $S$-basis of $\mathbb{P}$ and $t^{w} \in \mathbb{P}$ for all $w \in W_{\text {af }}^{0}$, we have

$$
\begin{aligned}
t^{w} & =\sum_{v \in W_{\mathrm{af}}^{0}} \bar{\xi}^{v}\left(t^{w}\right) j_{v} \\
& =\sum_{v \in W_{\mathrm{af}}^{0}} \bar{\xi}^{v}(w) j_{v}
\end{aligned}
$$

by the definition of $j_{v},(3.23)$, Lemma 3.24, and Proposition 3.42.

Let $D$ be the $W_{\text {af }}^{0} \times W_{\text {af }}^{0}$ matrix with $D_{v, w}=\bar{\xi}^{v}(w)$. It is invertible over $\mathrm{F}$ by $(3.58)$ and (3.61). Let $C=D^{-1}$.

Proposition 4.11. For every $u \in W_{\mathrm{af}}^{0}$ and $x \in W_{\mathrm{af}}$,

$$
j_{u}^{x}=\sum_{w \in W_{\mathrm{af}}^{0}} C_{w u} \bar{\xi}^{x}\left(t^{w}\right) .
$$

Remark 4.12. Note that by Propositions 4.11 and 4.15 below, the Schubert structure constants $d_{u v}^{w}$ for $H_{T}\left(\mathrm{Gr}_{G}\right)$ may be computed using only localizations of Schubert classes. 
Proof. Multiplying (4.24) by $C_{w u}$ and summing over $w \in W_{\text {af }}^{0}$ we have

$$
\begin{aligned}
\sum_{w \in W_{\mathrm{af}}^{0}} C_{w u} t^{w} & =\sum_{v, w \in W_{\mathrm{af}}^{0}} D_{v w} C_{w u} j_{v} \\
& =\sum_{v \in W_{\mathrm{af}}^{0}} \delta_{v u} j_{v} \\
& =j_{u} .
\end{aligned}
$$

Now take the coefficient of $A_{x}$.

Proposition 4.13. [131] For any antidominant $\lambda \in Q^{\vee}, t_{\lambda} \in W_{\text {af }}^{0}$ and

$$
j_{t_{\lambda}}=\sum_{\mu \in W \lambda} A_{t_{\mu}}
$$

Corollary 4.14. For any antidominant $\lambda \in Q^{\vee}$ and $x \in W_{\mathrm{af}}^{0}$, we have $x t_{\lambda} \in W_{\mathrm{af}}^{0}$ and

$$
j_{x t_{\lambda}}=j_{x} j_{t_{\lambda}}
$$

Proof. Follows from Proposition 4.13 and Theorem 4.10.

\subsection{Homology structure constants}

Recall that $\operatorname{Hom}_{S}\left(\Lambda_{\mathrm{Gr}}^{\prime}, S\right) \cong H_{T}(\mathrm{Gr})$.

For $u, v, w \in W_{\text {af }}^{0}$ define the $H_{T}(\mathrm{Gr})$ Schubert structure constants $d_{u v}^{w} \in S$ by

$$
j_{u} j_{v}=\sum_{w} d_{u v}^{w} j_{w}
$$

The $H_{T}(\mathrm{Gr})$-structure constants all appear in the expansion of the basis $\left\{j_{v} \mid v \in W_{\mathrm{af}}^{0}\right\}$ in terms of $A_{w}$. The following is due to Peterson [131].

Proposition 4.15. For $u, v, w \in W_{\mathrm{af}}^{0}$,

$$
d_{u v}^{w}= \begin{cases}j_{u}^{w v^{-1}} & \text { if } \ell\left(w v^{-1}\right)+\ell(v)=\ell(w) \\ 0 & \text { otherwise. }\end{cases}
$$

Proof. Using the notation of (4.23), $d_{u v}^{w}$ is the coefficient of $A_{w}$ in the expansion of $j_{u} j_{v}$ when the latter is written as a left $S$-linear combination of $\left\{A_{z} \mid z \in W_{\text {af }}\right\}$. Every product of the form $j_{u}^{x} A_{x} j_{v}^{y} A_{y}$ for $y \notin W_{\text {af }}^{0}$, is in the left $S$-module $\mathbb{A} A_{y}$ for $z \in W_{\text {af }}$, and by (3.11) $S A_{w} \cap \mathbb{A} A_{y}=0$. Therefore $d_{u v}^{w}$ is the coefficient of $A_{w}$ in $j_{u} A_{v}$, and applying (3.11) yields (4.29). 


\subsection{Peterson's "Quantum Equals Affine" Theorems}

The set $\mathcal{T}$ of the elements $\xi_{t_{\lambda}}$ for $\lambda \in Q^{\vee}$ antidominant, is multiplicatively closed since

$$
\xi_{x t_{\lambda}}=\xi_{x} \xi_{t_{\lambda}}
$$

for all such $\lambda$ and $x \in W_{\mathrm{af}}^{-}$. This follows from Corollary 4.14 and Theorem 4.10. For any $w \in W$ there is an antidominant $\lambda \in Q^{\vee}$ such that $w t_{\lambda} \in W_{\text {af }}^{-}$.

Let $Q H^{T}(G / B)$ be the $T$-equivariant quantum cohomology ring of $G / B$. Linearly it is isomorphic to $\mathbb{Z}\left[q_{i} \mid i \in I\right] \otimes_{\mathbb{Z}} H^{T}(G / B)$. For $\alpha^{\vee}=\sum_{i \in I} c_{i} \alpha_{i}^{\vee} \in Q^{\vee}$ define $q_{\alpha^{\vee}}=$ $\prod_{i \in I} q_{i}^{c_{i}} \in \mathbb{Z}\left[q_{i}^{ \pm} \mid i \in I\right]$. For $w \in W$ let $\sigma^{w}$ be the quantum Schubert class defined by the $w$-th opposite Schubert variety $\overline{B_{-} w B / B}$.

Theorem 4.16. [131] [88] Let $Q H^{T}(G / B)_{(q)}$ be the localization of $Q H^{T}(G / B)$ at the quantum parameters. Denote by $H_{T}\left(\mathrm{Gr}_{G}\right)_{\mathcal{T}}$ the localization of $H_{T}\left(\mathrm{Gr}_{G}\right)$ at the multiplicatively closed set $\mathcal{T}$. Then there is an $S$-algebra isomorphism $Q H^{T}(G / B)_{(q)} \rightarrow H_{T}\left(\mathrm{Gr}_{G}\right)_{\mathcal{T}}$ defined as follows. Given $\mu \in Q^{\vee}$ there is an antidominant $\lambda \in Q^{\vee}$ such that $w t_{\mu+\lambda} \in W_{\mathrm{af}}^{-}$. Then $q_{\mu} \sigma^{w} \mapsto \xi_{t_{\lambda}}^{-1} \xi_{w t_{\mu+\lambda}}$.

Remark 4.17. Since the Schubert bases of $Q H^{T}(G / B)$ and $H_{T}\left(\operatorname{Gr}_{G}\right)$ correspond, it follows that the Schubert structure constants for these rings agree. By Proposition 4.15, the equivariant Gromov-Witten invariants all have the form $j_{v}^{x}$.

There is also a parabolic version which covers all homogeneous spaces $G / P$.

Theorem 4.18. [131] [88] For any parabolic subgroup $P \subset G$, there is an ideal $J_{P} \subset$ $H_{T}(\mathrm{Gr})$ and a multiplicatively closed subset $\mathcal{T}_{P}$ of $H_{T}(\mathrm{Gr}) / J_{P}$, such that there is a ring isomorphism

$$
Q H^{T}(G / P)_{(q)} \cong\left(H_{T}(\mathrm{Gr}) / J_{P}\right)_{\mathcal{T}_{P}}
$$

where $Q H^{T}(G / P)$ is the T-equivariant small quantum cohomology ring of $G / P$ and $(q)$ is the set of quantum parameters.

Moreover, Schubert classes correspond to Schubert classes. In particular every Tequivariant Gromov-Witten invariant for any $G / P$, occurs as a Schubert structure constant for $H_{T}(\mathrm{Gr})$, and vice versa for $P=B$.

\section{A Appendix: Proof of coalgebra properties}

Let $M$ and $N$ be left $\mathrm{F}_{W}$-modules. Then $M, N, M \otimes_{\mathrm{F}} N$, and $\operatorname{Hom}_{\mathrm{F}}(M, N)$ are left F-modules. We define an $\mathrm{F}_{W}$-module structure on $M \otimes_{\mathrm{F}} N$ and $\operatorname{Hom}_{\mathrm{F}}(M, N)$. 
Proof of Proposition 3.14. We first check the well-definedness of the formula for $a \cdot m \otimes n$. Let $a \in \mathrm{F}_{W}$ with $\Delta(a)=\sum_{(a)} a_{(1)} \otimes a_{(2)}$. We further expand $a_{(k)}=\sum_{w} a_{(k)}^{w} w$ for $k=1,2$ where $a_{(k)}^{w} \in \mathrm{F}$. The condition of membership in $\operatorname{Im}(\Delta)$ of the right hand side of (3.33), is that only terms of the form $v \otimes v$ survive:

$$
\sum_{a} a_{(1)}^{v} a_{(2)}^{w}=0 \quad \text { for all } v \neq w
$$

We take a typical generator of the relations in $M \otimes_{Q} N: q m \otimes n-m \otimes q n$. We compute the componentwise action of $\Delta(a)$ on $q m \otimes n$ and $m \otimes q n$.

$$
\begin{aligned}
\Delta(a) \cdot(q m \otimes n) & =\sum_{a} a_{(1)} \cdot q m \otimes a_{(2)} \cdot n \\
& =\sum_{a, v, w} a_{(1)}^{v} a_{(2)}^{w} v \cdot q m \otimes w \cdot n \\
& =\sum_{a, v, w} a_{(1)}^{v} a_{(2)}^{w}(v \cdot q)(v \cdot m) \otimes w \cdot n \\
& =\sum_{a, v, w} a_{(1)}^{v} a_{(2)}^{w}(v \cdot q)(v \cdot m \otimes w \cdot n) .
\end{aligned}
$$

Similarly

$$
\Delta(a) \cdot(m \otimes q n)=\sum_{a, v, w} a_{(1)}^{v} a_{(2)}^{w}(w \cdot q)(v \cdot m \otimes w \cdot n) .
$$

The difference of these two expressions is

$$
\begin{aligned}
& \sum_{a} \sum_{v \neq w} a_{(1)}^{v} a_{(2)}^{w}(v \cdot q-w \cdot q)(v \cdot m \otimes w \cdot n) \\
= & \sum_{v \neq w}(v \cdot q-w \cdot q)(v \cdot m \otimes w \cdot n) \sum_{a} a_{(1)}^{v} a_{(2)}^{w} \\
= & 0 .
\end{aligned}
$$

Thus the formula for $a \cdot(m \otimes n)$ is well-defined.

Applying this to the special case of the action of $\mathrm{F}_{W}$ on $\mathrm{F}_{W} \otimes_{\mathrm{F}} \mathrm{F}_{W}$, we recover part (1), including (3.32). For $a, b \in \mathrm{F}_{W}$ we have

$$
\begin{aligned}
a \cdot(b \cdot(m \otimes n)) & =\sum_{(a)} \sum_{(b)} a_{(1)} \cdot\left(b_{(1)} \cdot m\right) \otimes a_{(2)} \cdot\left(b_{(2)} \cdot n\right) \\
& =\sum_{(a)} \sum_{(b)}\left(a_{(1)} b_{(1)}\right) \cdot m \otimes\left(a_{(2)} b_{(2)}\right) \cdot n \\
& =(a b) \cdot(m \otimes n)
\end{aligned}
$$


where the last step holds because of (3.32). Hence we have an action of $\mathrm{F}_{W}$ on $M \otimes_{\mathrm{F}} N$.

For the left $\mathrm{F}_{W}$-module $M$, the dual $M^{*}=\operatorname{Hom}_{\mathrm{F}}(M, \mathrm{~F})$ has a left $\mathrm{F}_{W}$-module structure defined by $w \cdot m^{*}=m^{*} \circ w^{-1}$ or more generally $a \cdot m^{*}=m^{*} \circ a^{t}$ for $w \in W$ and $a \in \mathrm{F}_{W}$. Consider the left F-linear isomorphism $M^{*} \otimes_{\mathrm{F}} N \cong \operatorname{Hom}_{\mathrm{F}}(M, N)$ given by $m^{*} \otimes n \mapsto(x \mapsto$ $\left.m^{*}(x) n\right)$. We define a left $\mathrm{F}_{W}$-module structure on $\operatorname{Hom}_{\mathrm{F}}(M, N)$ by declaring that the above map is an isomorphism of left $\mathrm{F}_{W}$-modules. It is enough to consider the action of $a=w: w \cdot m^{*} \otimes n=w \cdot m^{*} \otimes w \cdot n=m^{*} \circ w^{-1} \otimes w \cdot n$. This corresponds to the function $x \mapsto m^{*}\left(w^{-1} x\right) w \cdot n=w \cdot m^{*}\left(w^{-1} x\right) n$. If $f \in \operatorname{Hom}_{\mathrm{F}}(M, N)$ corresponds to $m^{*} \otimes n$ then the above function corresponds to $x \mapsto w \cdot f\left(w^{-1} x\right)$, which is the required action.

Proof of Proposition 3.16. We first compute

$$
\begin{aligned}
\Delta\left(A_{i}\right) & =\Delta\left(\alpha_{i}^{-1}\left(1-s_{i}\right)\right) \\
& =\alpha_{i}^{-1}\left(1 \otimes 1-s_{i} \otimes s_{i}\right) \\
& =\alpha_{i}^{-1}\left(1 \otimes 1-s_{i} \otimes 1+s_{i} \otimes 1-s_{i} \otimes s_{i}\right) \\
& =A_{i} \otimes 1+s_{i} \otimes A_{i} \\
& =\alpha_{i}^{-1}\left(1 \otimes 1-1 \otimes s+1 \otimes s_{i}-s_{i} \otimes s_{i}\right) \\
& =1 \otimes A_{i}+A_{i} \otimes s_{i} .
\end{aligned}
$$

This yields (3.35). It follows that the restriction of $\Delta$ to $\mathbb{A}$ has image in $\mathbb{A} \otimes_{S} \mathbb{A}$ and inherits the required properties by Proposition 3.14. All other assertions follow directly.

\section{B Appendix: Small torus GKM proofs}

Proof of Theorem 4.3. We prove (1) as (2) follows from it. There is a commutative diagram of ring homomorphisms

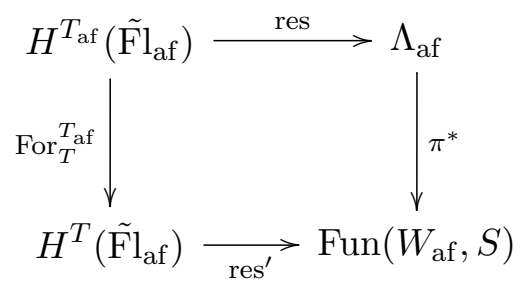

The horizontal maps are restriction to torus-fixed points. For $_{T}^{T_{\text {af }}}$ is the map that forgets from $T_{\text {af }}$-equivariance to $T$-equivariance. The top map is an isomorphism by Theorem 3.45. It is not hard to show that For is surjective and that $H^{T}\left(\tilde{\mathrm{F}} l_{\mathrm{af}}\right)$ has an $H^{T}(\mathrm{pt})$-basis given by the $T$-equivariant classes of Schubert varieties $\left[X^{v}\right]^{T} \in H^{T}\left(\tilde{\mathrm{F}} l_{\mathrm{af}}\right)$ for $v \in W_{\mathrm{af}}$. By commutativity of the diagram,

$$
\operatorname{Im}\left(\operatorname{res}^{\prime}\right)=\operatorname{Im}\left(\pi^{*}\right)=\bigoplus_{v \in W_{\mathrm{af}}} S \bar{\xi}^{v} .
$$


The functions $\bar{\xi}^{v}$ are independent since the matrix $\left(\pi\left(d_{v, w}\right)\right)_{v, w \in W_{\mathrm{af}}}$ is triangular with nonvanishing diagonal entries.

It remains to show that

$$
\operatorname{Im}\left(\pi^{*}\right)=\Lambda_{\mathrm{af}}^{\prime}
$$

Let $v \in W_{\text {af }}$. Certainly $\bar{\xi}^{v}$ satisfies (3.53) since $\xi^{v}$ does. We must check the conditions (4.1) and (4.2). Let $w \in W_{\text {af }}, \alpha \in \Phi$, and $d \in \mathbb{Z}_{>0}$. Let $W^{\prime} \subset W_{\text {af }}$ be the subgroup generated by $t_{\alpha} \vee$ and $r_{\alpha}$; it is isomorphic to the affine Weyl group of $S L_{2}$. Define the function $f: W^{\prime} \rightarrow S$ by $f(x)=\bar{\xi}^{v}(x w)$. Since $\xi^{v}$ satisfies (3.53) for $\mathrm{Fl}_{\mathrm{af}}, f$ satisfies (3.53) for the affine flag variety $\mathrm{Fl}^{\prime}$ corresponding to $\alpha$. It follows that $f$ is an $S$-linear combination of Schubert classes in $\mathrm{Fl}^{\prime}$. By Propositions B.1 and B.2 (proved below), $\pi \circ f$ satisfies (4.1) and (4.2), so that $\bar{\xi}^{v} \in \Lambda_{\mathrm{af}}^{\prime}$, as required.

Conversely, suppose $\xi \in \Lambda_{\text {af }}^{\prime}$. We show that

$$
\xi \in \bigoplus_{v \in W_{\mathrm{af}}} S \bar{\xi}^{v}
$$

Let $x=t_{\lambda} u$ be of minimal length in the support of $\xi$, with $u \in W$ and $\lambda \in Q^{\vee}$. It suffices to show that

$$
\xi(x) \in \bar{\xi}^{x}(x) S \text {. }
$$

Suppose (B.5) holds. Define $\xi^{\prime}: W_{\text {af }} \rightarrow S$ by $\xi^{\prime}=\xi-\left(\xi(x) / \bar{\xi}^{x}(x)\right) \bar{\xi}^{x}$. Since $\Lambda_{\text {af }}^{\prime}$ is an $S$-module, $\xi^{\prime} \in \Lambda_{\text {af }}^{\prime}$. Moreover $\Omega\left(\xi^{\prime}\right) \supsetneq \Omega(\xi)$ where $\Omega(\xi)$ is defined by (3.64). By induction (B.4) holds for $\xi^{\prime}$ and therefore it holds for $\xi$.

We now show (B.5). The elements $\left\{\alpha \mid \alpha \in \Phi^{+}\right\}$are relatively prime in $S$. Letting $\alpha \in \Phi^{+}$, by (3.61) it suffices to show that $\xi(x) \in J:=\alpha^{d} S$ where $d=\left|\operatorname{Inv}_{\alpha}\left(x^{-1}\right)\right|$ and $\operatorname{Inv}_{\alpha}\left(x^{-1}\right)$ is the set of roots in $\operatorname{Inv}\left(x^{-1}\right)$ (see (2.21)) of the form $\pm \alpha+k \delta$ for some $k \in \mathbb{Z}_{\geq 0}$.

Note that for $\beta \in \Phi_{\mathrm{af}}^{+\mathrm{re}}, \beta \in \operatorname{Inv}\left(x^{-1}\right)$ if and only if $x^{-1} \cdot \beta \in-\Phi_{\mathrm{af}}^{+\mathrm{re}}$. We have

$$
x^{-1} \cdot( \pm \alpha+k \delta)=u^{-1} t_{-\lambda} \cdot( \pm \alpha+k \delta)= \pm u^{-1} \alpha+(k \pm\langle\lambda, \alpha\rangle) \delta .
$$

Letting $\chi=\chi\left(\alpha \in \operatorname{Inv}\left(u^{-1}\right)\right)$ we have

$$
\operatorname{Inv}_{\alpha}\left(x^{-1}\right)= \begin{cases}\{\alpha, \alpha+\delta, \ldots, \alpha-(\langle\lambda, \alpha\rangle+1-\chi) \delta\} & \text { if }\langle\lambda, \alpha\rangle \leq 0 \\ \{-\alpha+\delta,-\alpha+2 \delta, \ldots,-\alpha+(\langle\lambda, \alpha\rangle-\chi) \delta\} & \text { if }\langle\lambda, \alpha\rangle>0\end{cases}
$$

Suppose first that $\langle\lambda, \alpha\rangle>0$. Then $d=\langle\lambda, \alpha\rangle-\chi\left(\alpha \in \operatorname{Inv}\left(u^{-1}\right)\right)$. Applying (4.2) to $y=t_{(1-d) \alpha^{\vee}} x$, we have $Z_{1} \in J$ where

$$
\begin{aligned}
Z_{1} & =\xi\left(\left(1-t_{\alpha \vee}\right)^{d-1}\left(1-s_{\alpha}\right) y\right) \\
& =(-1)^{d-1} \xi\left(\left(1-t_{-\alpha \vee}\right)^{d-1} x\right)-\xi\left(\left(1-t_{\alpha} \vee\right)^{d-1} s_{\alpha} y\right) \\
& =(-1)^{d-1} \xi(x)-\xi\left(\left(1-t_{\alpha}\right)^{d-1} s_{\alpha} y\right)
\end{aligned}
$$


where the last equality holds by the assumption on $\operatorname{Supp}(\xi)$ and a calculation of $\operatorname{Inv}_{\alpha}\left(\left(s_{\alpha+d \delta} x\right)^{-1}\right)$, giving $x>s_{\alpha+d \delta} x>t_{-k \alpha \vee} x$ for all $1 \leq k \leq d-1$. By Lemma 4.1 we have $Z_{2} \in J$ where

$$
Z_{2}=\xi\left(\left(1-t_{\alpha \vee}\right)^{d-1} s_{\alpha} y\right)-\xi\left(\left(1-t_{\alpha \vee}\right)^{d-1} t_{p \alpha} s_{\alpha} y\right)
$$

for any $p \in \mathbb{Z}$. Thus

$$
Z_{1}+Z_{2}=(-1)^{d-1} \xi(x)-\xi\left(\left(1-t_{\alpha}\right)^{d-1} t_{p \alpha} s_{\alpha} y\right) \in J
$$

By the assumption on $\operatorname{Supp}(x)$ and (B.6),

$$
\xi\left(\left(1-t_{\alpha^{\vee}}\right)^{d-1} t_{p \alpha \vee} s_{\alpha} y\right)=0
$$

for $p=2-d$. It follows that $\xi(x) \in J$.

Otherwise $\langle\lambda, \alpha\rangle \leq 0$. By the previous case we may assume that $t_{d \alpha} \vee x \notin \operatorname{Supp}(\xi)$. Thus

$$
\xi(x)=\xi\left(\left(1-t_{\alpha^{\vee}}\right)^{d} x\right) \in J
$$

by induction on $\operatorname{Supp}(\xi)$ and (4.1). This proves (B.5) and (B.4) as required.

\section{B.1 Small torus GKM condition for $\hat{\mathrm{sl}}_{2}$}

In this section we consider the root datum for $\hat{\mathrm{sl}}_{2}$. Let $\Phi^{+}=\{\alpha\}$ where $\alpha=\alpha_{1}$. Also $\delta=\alpha_{0}+\alpha_{1}$ so that $\pi\left(\alpha_{0}\right)=-\pi\left(\alpha_{1}\right)$ and the level zero action of $W_{\text {af }}$ is given by $s_{0} \cdot \alpha=$ $s_{1} \cdot \alpha=-\alpha$. For $i \in \mathbb{Z}_{\geq 0}$ let

$$
\begin{aligned}
\sigma_{2 i} & =\left(s_{1} s_{0}\right)^{i} & \sigma_{-2 i} & =\left(s_{0} s_{1}\right)^{i}, \\
\sigma_{2 i+1} & =s_{0} \sigma_{2 i} & \sigma_{-(2 i+1)} & =s_{1} \sigma_{-2 i} .
\end{aligned}
$$

Then we have $\ell\left(\sigma_{j}\right)=|j|$ for $j \in \mathbb{Z}, W_{\text {af }}^{I}=\left\{\sigma_{j} \mid j \in \mathbb{Z}_{\geq 0}\right\}$, and

$$
\sigma_{2 i}=t_{-i \alpha^{\vee}} \quad \text { for } i \in \mathbb{Z} .
$$

Let $\xi_{j}^{i}:=\pi\left(\xi^{\sigma_{i}}\left(\sigma_{j}\right)\right)$ for $i, j \in \mathbb{Z}$. For $m, a \in \mathbb{Z}_{\geq 0}$,

$$
\xi_{j}^{m}=(-1)^{m j}\left(\begin{array}{c}
m+a \\
m
\end{array}\right) \alpha^{m}
$$

where $j \in\{m+2 a, m+2 a+1,-m-2 a-1,-m-2 a-2\}$. This is easily proved by induction using (3.60). The rest of the values for $\xi^{m}$ are zero by (3.58). For $m<0$ we may use

$$
\xi_{j}^{m}=(-1)^{m} \xi_{-j}^{-m} \quad \text { for } m, j \in \mathbb{Z}
$$

which follows from the Dynkin symmetry $0 \leftrightarrow 1$. 
Proposition B.1. For all $d \geq 1, m \in \mathbb{Z}$, and $w \in W_{\text {af }}$ we have

$$
\xi^{m}\left(\left(1-t_{\alpha \vee}\right)^{d} w\right) \in \alpha^{d} \mathbb{Z}[\alpha]
$$

Proof. One may reduce to $m \geq 0$ using (B.9). Equation (B.10) is proved for $m=2 i$, $w=t_{(-i-a) \alpha^{\vee}}$ and $d=(i+a)+(i+1+b)=2 i+a+b+1$ for $a, b \in \mathbb{Z}_{\geq 0}$, as the other possibilities are similar or easier. Equation (B.10) can be rewritten as

$$
Z:=\sum_{k=0}^{d}(-1)^{k}\left(\begin{array}{l}
d \\
k
\end{array}\right) \xi_{-2 i-2-2 b+2 k}^{2 i} \in \alpha^{d} \mathbb{Z}[\alpha] .
$$

By $(3.58) \xi_{2 p}^{2 i}=0$ for $-2 i-2<2 p<2 i$. Using (B.8),

$$
\begin{aligned}
Z & =\left(\sum_{k=0}^{b}+\sum_{k=2 i+1+b}^{d}\right)(-1)^{k}\left(\begin{array}{l}
d \\
k
\end{array}\right) \xi_{-2 i-2-2 b+2 k}^{2 i} \\
& =\sum_{k=0}^{b}(-1)^{k}\left(\begin{array}{l}
d \\
k
\end{array}\right) \xi_{-2 i-2-2 b+2 k}^{2 i}+\sum_{k=0}^{a}(-1)^{k+2 i+1+b}\left(\begin{array}{c}
d \\
2 i+1+b+k
\end{array}\right) \xi_{2 i+2 k}^{2 i} \\
& =(-1)^{b} \sum_{k=0}^{b}(-1)^{k}\left(\begin{array}{c}
d \\
b-k
\end{array}\right) \xi_{-2 i-2-2 k}^{2 i}-(-1)^{b} \sum_{k=0}^{a}(-1)^{k}\left(\begin{array}{c}
d \\
a-k
\end{array}\right) \xi_{2 i+2 k}^{2 i} \\
& =(-1)^{b} \sum_{k=0}^{b}(-1)^{k}\left(\begin{array}{c}
d \\
b-k
\end{array}\right)\left(\begin{array}{c}
2 i+k \\
2 i
\end{array}\right) \alpha^{2 i}-(-1)^{b} \sum_{k=0}^{a}(-1)^{k}\left(\begin{array}{c}
d \\
a-k
\end{array}\right)\left(\begin{array}{c}
2 i+k \\
2 i
\end{array}\right) \alpha^{2 i}
\end{aligned}
$$

Taking the coefficient of $(-x)^{b}$ in $(1-x)^{d} /(1-x)^{2 i+1}=(1-x)^{a+b}$ we have

$$
\sum_{k=0}^{b}(-1)^{k}\left(\begin{array}{c}
d \\
b-k
\end{array}\right)\left(\begin{array}{c}
2 i+k \\
2 i
\end{array}\right)=\left(\begin{array}{c}
a+b \\
b
\end{array}\right) .
$$

Exchanging the roles of $a$ and $b$ we see that $Z=0$ which implies (B.11).

Proposition B.2. For all $d \geq 1, m \in \mathbb{Z}$, and $w \in W_{\text {af }}$ we have

$$
\xi^{m}\left(\left(1-t_{\alpha}\right)^{d-1}\left(1-s_{\alpha}\right) w\right) \in \alpha^{d} \mathbb{Z}[\alpha] .
$$

Proof. Let $y=\left(1-t_{\alpha^{\vee}}\right)^{d-1}$. Without loss of generality we may assume $w=t_{p \alpha^{\vee}}$ for some $p \in \mathbb{Z}$. By Proposition B.1, $\xi^{m}$ satisfies (4.1). We have

$$
\begin{aligned}
\xi^{m}\left(y\left(1-s_{\alpha}\right) t_{p \alpha}\right) & =\xi^{m}\left(y t_{p \alpha^{\vee}}\right)-\xi^{m}\left(y t_{-p \alpha^{\vee}} s_{\alpha}\right) \\
& \equiv \xi^{m}(y)-\xi^{m}\left(y s_{\alpha}\right) \quad \bmod \alpha^{d} \mathbb{Z}[\alpha]
\end{aligned}
$$


using Lemma 4.1 twice. However

$$
\begin{aligned}
\xi^{m}(y)-\xi^{m}\left(y s_{\alpha}\right) & =\xi^{m}\left(y \alpha A_{\alpha}\right) \\
& =(y \cdot \alpha)\left(A_{\alpha} \bullet \xi^{m}\right)(y) .
\end{aligned}
$$

Now $y \cdot \alpha= \pm \alpha$ and $A_{\alpha} \bullet \xi^{m}$ is 0 if $m \geq 0$ and is equal to $\xi^{m+1}$ if $m<0$. Assuming the latter, by (4.1) for $d-1, \xi^{m+1}(y) \in \alpha^{d-1} S$, so that $\xi^{m}(y)-\xi^{m}\left(y s_{\alpha}\right) \in \alpha^{d} S$ as required.

\section{Appendix: Homology of Gr}

Let $K$ be the maximal compact form of $G$ and $T_{\mathbb{R}}=K \cap T$. The based loop group $\Omega K$ of continuous maps $\left(S^{1}, 1\right) \rightarrow(K, 1)$, has a $T_{\mathbb{R}}$-equivariant multiplication map $\Omega K \times$ $\Omega K \rightarrow \Omega K$ given by pointwise multiplication on $K$, and this induces the structure of a commutative and co-commutative Hopf-algebra on $H^{T_{\mathbb{R}}}(\Omega K)$. The co-commutativity of $H^{T_{\mathbb{R}}}(\Omega K)$ follows from the fact that $\Omega K$ is a homotopy double-loop space.

$\mathrm{Gr}=\mathrm{Gr}_{G}$ and $\Omega K$ are weakly homotopy equivalent [136]. By "fattening loops", it follows that $H_{T}(\mathrm{Gr})$ and $H^{T}(\tilde{\mathrm{Gr}})$ are dual Hopf algebras over $S=H^{T}(\mathrm{pt})$ with duality given by an intersection pairing

$$
\langle\cdot, \cdot\rangle: H_{T}(\mathrm{Gr}) \times H^{T}(\tilde{\mathrm{Gr}}) \rightarrow S .
$$

We may regard an element $\xi \in H_{T}(\mathrm{Gr})$ as an $S$-module homomorphism $H^{T}(\tilde{\mathrm{Gr}}) \rightarrow S$ by $\xi(f)=\langle\xi, f\rangle$.

Lemma C.1. Let $\lambda, \mu \in Q^{\vee}$, and consider the maps $i_{\lambda}^{*}, i_{\mu}^{*}: H^{T}(\tilde{\mathrm{Gr}}) \rightarrow S$ as elements of $H_{T}(\mathrm{Gr})$. Then in $H_{T}(\mathrm{Gr})$, we have

$$
i_{\lambda}^{*} i_{\mu}^{*}=i_{\lambda+\mu}^{*}
$$

Proof. It suffices to work in $H^{T_{\mathbb{R}}}(\Omega K)$. The map $i_{\lambda}^{*} i_{\mu}^{*}$ is induced by the map pt $\rightarrow$ $\Omega K \times \Omega K \rightarrow \Omega K$ where the image of the first map is the pair $\left(t_{\lambda}, t_{\mu}\right) \in \Omega K \times \Omega K$ of fixed points, and the latter map is multiplication. Treating $t_{\lambda}, t_{\mu}: S^{1} \rightarrow K$ as homomorphisms into $K$, the pointwise multiplication of $t_{\lambda}$ and $t_{\mu}$ gives $t_{\lambda+\mu}$. Thus $i_{\lambda}^{*} i_{\mu}^{*}=i_{\lambda+\mu}^{*}$.

The antipode of $H_{T}\left(\mathrm{Gr}_{G}\right)$ is given by $i_{\lambda}^{*} \mapsto i_{-\lambda}^{*}$, since the fixed points satisfy $t_{\lambda}^{-1}=t_{-\lambda}$ in $\Omega K$.

For $w \in W_{\text {af }}^{0}$ denote by $\left[X_{w}\right]_{T} \in H_{T}(\mathrm{Gr})$ the equivariant fundamental homology class of the Schubert variety $X_{w}:=\overline{B_{\mathrm{af}} \dot{w} P_{\mathrm{af}} / P_{\mathrm{af}}} \subset G_{\mathrm{af}} / P_{\mathrm{af}}=\mathrm{Gr}$.

Using the intersection pairing we have that $\left\{\left[X_{w}\right] \mid w \in W_{\mathrm{af}}^{0}\right\}$ is the basis of $H_{T}\left(\mathrm{Gr}_{G}\right)$ that is dual to the basis $\left\{\left[X^{w}\right]^{T} \mid w \in W_{\text {af }}^{0}\right\}$ of $H^{T}$ ( $\left.\tilde{\mathrm{Gr}}\right)$, which corresponds to the basis $\left\{\bar{\xi}^{w} \mid w \in W_{\mathrm{af}}^{0}\right\}$ of $\Lambda_{\mathrm{Fl}}^{\prime}$ under the isomorphism of Theorem 4.3.

Proof of Prop. 4.6. Follows from the above discussion. 


\section{Bibliography}

[1] S. Agnihotri, Quantum cohomology and the Verlinde algebra, Ph.D. Thesis, University of Oxford, 1995.

[2] H. H. Andersen, J. C. Jantzen, W. Soergel, Representations of quantum groups at a pth root of unity and of semisimple groups in characteristic p: independence of $p$, Astérisque No. 220 (1994), 321 pp.

[3] A. Arabia, Cycles de Schubert et cohomologie équivariante de $K / T$, Invent. Math. 85 (1986), no. 1, 39-52.

[4] S. Assaf, A combinatorial realization of Schur-Weyl duality via crystal graphs and dual equivalence graphs, 20th Annual International Conference on Formal Power Series and Algebraic Combinatorics (FPSAC 2008), pp. 141-152, Discr. Math. Theor. Comput. Sci. Proc., AJ, Assoc. Discrete Math. Theor. Comput. Sci., Nancy, France, 2008.

[5] S. Assaf, Dual Equivalence Graphs I: A combinatorial proof of LLT and Macdonald positivity, preprint arXiv:1005.3759.

[6] S. Assaf, S. Billey, Affine dual equivalence and k-Schur functions, J. Comb. 3 (2012), no. 3, 343-399.

[7] J. Bandlow, A. Schilling, M. Zabrocki, The Murnaghan-Nakayama rule for k-Schur functions, J. of Comb. Th., Ser. A Volume 118, Issue 5, June 2011, 1588-1607.

[8] L. Bégin, P. Mathieu, M.A. Walton, ŝu(3) $k$ fusion coefficients, Mod. Phys. Lett. A 7 (1995), no. 35, 3255-3265.

[9] L. Bégin, A. Kirillov, P. Mathieu, M.A. Walton, Berenstein-Zelevinsky triangles, elementary couplings and fusion rules, Lett. Math. Phys. 28 (1993), 257-268.

[10] E. Bender, D.E. Knuth, Enumeration of plane partitions, J. Comb. Theory. Ser. A. 13 (1972) 40-54. 
[11] C. Berg, N. Bergeron, H. Thomas, M. Zabrocki, Expansion of $k$-Schur functions for maximal $k$-rectangles within the affine nilCoxeter algebra, J. Comb. 3 (2012), no. 3, $563-589$.

[12] C. Berg, B. C. Jones, M. Vazirani, A bijection on core partitions and a parabolic quotient of the affine symmetric group, J. Combin. Theory Ser. A 116 (8) (2009) $1344-1360$.

[13] C. Berg, F. Saliola, L. Serrano, Pieri operators on the affine nilCoxeter algebra, preprint arXiv: 1203.4465.

[14] C. Berg, F. Saliola, L. Serrano, Combinatorial expansions for families of noncommutative $k$-Schur functions, preprint arXiv:1208.4857.

[15] N. Bergeron, F. Sottile, Skew Schubert functions and the Pieri formula for flag manifolds, Trans. Amer. Math. Soc. 354 (2002), no. 2, 651-673.

[16] A. Bertram, I. Ciocan-Fontanine, W. Fulton, Quantum multiplication of Schur polynomials, J. Algebra, 219(2) (1999), 728-746.

[17] S. Billey, Kostant Polynomials and the Cohomology Ring for G/B, Duke Math. J. 96 (1999), 205-224.

[18] S. Billey, W. Jockusch, R. Stanley, Some combinatorial properties of Schubert polynomials, J. Algebraic Combin. 2 (1993), 345-374.

[19] S. Billey, M. Haiman, Schubert polynomials for the classical groups, J. Amer. Math. Soc. 8 (1995), no. 2, 443-482.

[20] S. Billey, T.K. Lam, Vexillary elements in the hyperoctahedral group, J. Algebraic Combin. 8 (1998), no. 2, 139-152.

[21] A. Björner, F. Brenti, Affine permutations of type A, Electronic Journal of Combinatorics 3, (1996), Research paper 18.

[22] R. Bott, The space of loops on a Lie group, Michigan Math Journal, 5 (1958), 35-61.

[23] D. Bravo, L. Lapointe, A recursion formula for $k$-Schur functions, J. Combin. Theory Ser. A 116 (2009), no. 4, 918-935.

[24] F. Brenti, S. Fomin, A. Postnikov, Mixed Bruhat operators and Yang-Baxter equations for Weyl groups, Internat. Math. Res. Notices 1999, no. 8, 419-441.

[25] J. Brichard, The Center of the Nilcoxeter and 0-Hecke Algebras, preprint arXiv:0811.2590. 
[26] A. Buch, Stanley symmetric functions and quiver varieties, J. Algebra 235 (2001), no. $1,243-260$.

[27] A. Buch, A Littlewood-Richardson rule for the K-theory of Grassmannians, Acta Math. 189 (2002), no. 1, 37-78.

[28] A. Buch, A. Kresch, M. Shimozono, H. Tamvakis, A. Yong, Stable Grothendieck polynomials and $K$-theoretic factor sequences, Math. Annalen 340 (2008), 359-382.

[29] A. S. Buch, A. Kresch, H. Tamvakis, Gromov-Witten invariants on Grassmannians, J. Amer. Math. Soc. 16 (2003), no. 4, 901-915.

[30] P.-E. Chaput, L. Manivel, N. Perrin, Affine symmetries of the equivariant cohomology ring of rational homogeneous spaces, Math. Res. Letters 16 (2009), 7-21.

[31] I. Cherednik, Double affine Hecke algebras, London Mathematical Society Lecture Note Series, 319. Cambridge University Press, Cambridge, 2005.

[32] L.-C. Chen, M. Haiman, A representation-theoretic model for $k$-atoms, Talk 1039-05169 at the AMS meeting in Claremont, McKenna, May 2008.

[33] I. Coskun, A Littlewood-Richardson rule for two-step flag varieties, Invent. Math. 176 (2009), no. 2, 325-395.

[34] C.J. Cummins, su(n) and sp(2n) WZE fusion rules, J. Phys. A 24 (1991), no. 2, 391-400.

[35] A. J. Dalal, J. Morse, The ABC's of affine Grassmannians and Hall-Littlewood polynomials, DMTCS proc. AR (2012) 935-946.

[36] A. J. Dalal, J. Morse, A t-generalization for Schubert representatives of the affine Grassmannian, preprint (2013).

[37] M. Demazure, Désingularization des variétés de Schubert, Annales E.N.S, 6 (1974) $53-88$.

[38] T. Denton, Canonical decompositions of affine permutations, affine codes, and split $k$-Schur functions, Electron. J. Combin. 19 (2012), no. 4, Paper 19, 41 pp.

[39] F. Descouens, H. Morita, Factorization formulas for Macdonald polynomials, Europ. J. of Comb., Volume 29, Issue 2, February 2008, pp. 395-410.

[40] P. Edelman, C. Greene, Balanced tableaux, Adv. Math. 63 (1987), 42-99.

[41] H. Eriksson, K. Eriksson, Affine Weyl groups as infinite permutations, Electron. J. Combin. 5 (1998) Research Paper 18, 32 pp. (electronic). 
[42] S. Fomin, Schensted algorithms for dual graded graphs, J. Algebraic Combin., 4 (1995), no. 1 , pp. $5-45$.

[43] S. Fomin, S. Gelfand, A. Postnikov, Quantum Schubert polynomials, J. Amer. Math. Soc. 10 (1997), no. 3, 565-596.

[44] S. Fomin, C. Greene, Noncommutative Schur functions and their applications, Discrete Mathematics 193 (1998), 179-200. Reprinted in the Discrete Math Anniversary Volume 306 (2006), 1080-1096.

[45] S. Fomin, A.N. Kirillov, Grothendieck polynomials and the Yang-Baxter equation, Proc. 6th Intern. Conf. on Formal Power Series and Algebraic Combinatorics, DIMACS, 1994, 183-190.

[46] S. Fomin, A.N. Kirillov, Combinatorial $B_{n}$-analogues of Schubert polynomials, Trans. Amer. Math. Soc. 348 (1996), no. 9, 3591-3620.

[47] S. Fomin, N. Reading, Root systems and generalized associahedra, Lecture notes for the IAS/Park City Graduate Summer School in Geometric Combinatorics.

[48] S. Fomin, R. Stanley, Schubert polynomials and the nilCoxeter algebra, Advances in Math. 103 (1994), 196-207.

[49] W. Fulton, C. Woodward, On the quantum product of Schubert classes, J. Algebraic Geom. 13 (2004), 641-661.

[50] A. Garsia, M. Haiman, Some natural bigraded $S_{n}$-Modules and $q$, $t$-Kostka coefficients, Elect. J. of Comb., R24, Volume 3(2), 1996. 60 pages.

[51] A. Garsia, C. Procesi, On certain graded Sn-modules and the q-Kostka polynomials, Adv. Math. 94 (1992), no. 1, 82-138.

[52] A. Garsia, J. Remmel, Plethystic formulas and positivity for q,t-Kostka coefficients, Mathematical essays in honor of Gian-Carlo Rota (Cambridge, MA, 1996), 245-262, Progr. Math., 161, Birkhäuser Boston, Boston, MA, 1998.

[53] A. Garsia, G. Tesler, Plethystic formulas for Macdonald q,t-Kostka coefficients, Adv. Math. 123 (1996), no. 2, 144-222.

[54] V. Ginzburg, Perverse sheaves on a loop group and Langlands' duality, preprint math. AG/9511007.

[55] V. Ginzburg, Geometric methods in the representation theory of Hecke algebras and quantum groups. Notes by Vladimir Baranovsky., NATO Adv. Sci. Inst. Ser. C Math. Phys. Sci., 514, Representation theories and algebraic geometry (Montreal, PQ, 1997), 127-183, Kluwer Acad. Publ., Dordrecht, 1998. 
[56] F. Goodman, H. Wenzl, Littlewood-Richardson coefficients for Hecke algebras at roots of unity, Adv of Math, 82 (1990) 244-265.

[57] M. Goresky, R. Kottwitz, R. MacPherson, Equivariant cohomology, Koszul duality, and the localization theorem, Invent. Math. 131 (1998), no. 1, 25-83.

[58] M. Goresky, R. Kottwitz, R. MacPherson, Homology of affine Springer fibers in the unramified case, Duke Math. J. 121 (2004), 509-561.

[59] W. Graham, Positivity in equivariant Schubert calculus, Duke Math. J. 109 (2001), no. 3, 599-614.

[60] M. Haiman, Macdonald polynomials and geometry, New Perspectives in Geometric Combinatorics, MSRI Publications 37 (1999), 207-254.

[61] M. Haiman, Hilbert schemes, polygraphs, and the Macdonald positivity conjecture, J. Amer. Math. Soc. 14 (2001), 941-1006.

[62] M. Haiman, Dual equivalence with applications, including a conjecture of Proctor, Discrete Mathematics 99 (1992), 79-113.

[63] Z. Hamaker, B. Young, Relating Edelman-Greene insertion to the Little map, preprint arXiv:1210.7119.

[64] J. E. Humphreys, Reflection groups and Coxeter groups, Cambridge Studies in Advanced Mathematics, 29. Cambridge University Press, Cambridge, 1990. xii+204 pp.

[65] T. Ikeda, L. Mihalcea, H. Naruse, Double Schubert polynomials for the classical groups, Adv. Math. 226 (2011), no. 1, 840-886.

[66] N. Jing, Vertex operators and Hall-Littlewood symmetric functions, Adv. Math. 87 (1991) 226-248.

[67] V.G. Kac, Infinite-dimensional Lie algebras, Third edition. Cambridge University Press, Cambridge, 1990. xxii+400 pp. ISBN: 0-521-37215-1.

[68] M. Kashiwara, The flag manifold of Kac-Moody Lie algebra, Algebraic analysis, geometry, and number theory (Baltimore, MD, 1988), 161-190, Johns Hopkins Univ. Press, Baltimore, MD, 1989.

[69] M. Kashiwara, M. Shimozono, Equivariant K-theory of affine flag manifolds and affine Grothendieck polynomials, Duke Math. J. 148 (2009), no. 3, 501-538.

[70] A. N. Kirillov, M. Noumi, q-difference raising operators for Macdonald polynomials and the integrality of transition coefficients. Algebraic methods and $q$-special functions (Montréal, QC, 1996), 227-243, CRM Proc. Lecture Notes, 22, Amer. Math. Soc., Providence, RI, 1999. 
[71] F. Knop, Integrality of two variable Kostka functions, J. Reine Angew. Math. 482 (1997), 177-189.

[72] D. Knuth, Permutations, matrices, and generalized Young tableaux, Pac. J. Math. 34 (1970), 709-727.

[73] A. Knutson, T. Lam, D. Speyer, Positroid varieties I: juggling and geometry, preprint arXiv:0903.3694.

[74] A. Knutson, T. Tao, Puzzles and (equivariant) cohomology of Grassmannians, Duke Math. J. 119 (2003), no. 2, 221-260.

[75] B. Kostant, S. Kumar, The nil Hecke ring and cohomology of G/P for a Kac-Moody group G, Adv. in Math. 62 (1986), no. 3, 187-237.

[76] B. Kostant, S. Kumar, T-equivariant K-theory of generalized flag varieties, J. Differential Geom. 32 (1990), no. 2, 549-603.

[77] S. Kumar, Kac-Moody groups, their flag varieties and representation theory, Progress in Mathematics, 204. Birkhäuser Boston, Inc., Boston, MA, 2002. xvi+606 pp.

[78] T. Lam, Affine Stanley symmetric functions, Amer. J. Math. 128 (2006), no. 6, 15531586.

[79] T. Lam, Schubert polynomials for the affine Grassmannian, J. Amer. Math. Soc. 21 (2008), no. 1, 259-281.

[80] T. Lam, Affine Schubert classes, Schur positivity, and combinatorial Hopf algebras, Bull. Lond. Math. Soc. 43 (2011), no. 2, 328-334.

[81] T. Lam, L. Lapointe, J. Morse, M. Shimozono, Affine insertion and Pieri rules for the affine Grassmannian, Mem. Amer. Math. Soc. 208 (2010), no. 977, xii+82 pp. ISBN: 978-0-8218-4658-2.

[82] T. Lam, L. Lapointe, J. Morse, M. Shimozono, $k$-shape poset and branching of $k$-Schur functions, Mem. Amer. Math. Soc., posted October 16, 2012. ISSN 1947-6221(online) ISSN 0065-9266(print) arXiv: 1007.5334.

[83] T. Lam, C. Li, L. Mihalcea, M. Shimozono, Quantum K-theory of $G / B$ and $K-$ homology of affine Grassmannians, in preparation.

[84] T. Lam, A. Schilling, M. Shimozono, Schubert Polynomials for the affine Grassmannian of the symplectic group, Math. Z. 264, (2010), 765-811.

[85] T. Lam, A. Schilling, M. Shimozono, K-theoretic Schubert calculus of the affine Grassmannian, Compositio Mathematica 146 Issue 4 (2010) 811-852. 
[86] T. Lam, M. Shimozono, A Little bijection for affine Stanley symmetric functions, Seminaire Lotharingien de Combinatoire 54A (2006), B54Ai.

[87] T. Lam, M. Shimozono, Dual graded graphs for Kac-Moody algebras, Algebra and Number Theory 1 (2007), 451-488.

[88] T. Lam, M. Shimozono, Quantum cohomology of $G / P$ and homology of affine Grassmannian, Acta. Math. 204 (2010), 49-90.

[89] T. Lam, M. Shimozono, k-Double Schur functions and equivariant (co)homology of the affine Grassmannian, Math. Ann. 356 (2013), no. 4, 1379-1404.

[90] T. Lam, M. Shimozono, From double quantum Schubert polynomials to k-double Schur functions via the Toda lattice, preprint arXiv:1109.2193.

[91] L. Lapointe, J. Morse, Tableaux statistics for two part Macdonald polynomials, Algebraic combinatorics and quantum groups, 61-84, World Sci. Publ., River Edge, NJ, 2003 .

[92] L. Lapointe, A. Lascoux, J. Morse, Tableau atoms and a new Macdonald positivity conjecture, Duke Math. J. 116 (2003), no. 1, 103-146.

[93] L. Lapointe, J. Morse, Schur function analogs for a filtration of the symmetric function space, J. Comb. Th. A 101 (2) (2003) pp. 191-224.

[94] L. Lapointe, J. Morse, Schur function identities, their t-analogs, and $k$-Schur irreducibility, Advances in Mathematics 180 (2003), no. 1, 222-247.

[95] L. Lapointe, J. Morse, Tableaux on $k+1$-cores, reduced words for affine permutations, and k-Schur expansions, J. Combin. Theory Ser. A 112 (2005), no. 1, 44-81.

[96] L. Lapointe, J. Morse, A k-tableau characterization of k-Schur functions, Adv. Math. 213 (2007), no. 1, 183-204.

[97] L. Lapointe, J. Morse, Quantum cohomology and the $k$-Schur basis, Trans. Amer. Math. Soc. 360 (2008), pp. 2021-2040.

[98] L. Lapointe, J. Morse, M. Wachs, Type A-affine Weyl group and the $k$-Schur functions, unpublished.

[99] L. Lapointe, M.-E. Pinto, Charge on tableaux and the poset of $k$-shapes, preprint (2013), arXiv:1305.2438.

[100] L. Lapointe, L. Vinet, A short proof of the integrality of the Macdonald (q,t)-Kostka coefficients, Duke Math. J. Volume 91, Number 1 (1998), 205-214. 
[101] A. Lascoux, Anneau de Grothendieck de la varieté de drapeaux, In "The Grothendieck Festschrift,volume III" of Progr Math, Boston, Birkhäuser, (1990) 1-34.

[102] A. Lascoux, Classes de Chern des variétés de drapeaux, C. R. Acad. Sci. Paris Sér. I Math. 295 (1982), no. 5, 393-398.

[103] A. Lascoux, Ordering the affine symmetric group, in Algebraic Combinatorics and Applications (Gossweinstein, 1999), 219-231, Springer, Berlin (2001).

[104] A. Lascoux, B. Leclerc, J.-Y. Thibon, Fonctions de Hall-Littlewood et Polynômes de Kostka-Foulkes aux racines de l'unité, Comptes Rendus de l'Academie des Sciences, Paris, 316 (1993), pp. 1-6.

[105] A. Lascoux, B. Leclerc, J.-Y. Thibon, Crystal graphs and q-analogues of weight multiplicities for the root system $A_{n}$, Lett. Math. Phys. 35 (1995), no. 4, 359-374.

[106] A. Lascoux, B. Leclerc, J.-Y. Thibon, "The plactic monoid" in Algebraic Combinatorics on Words, M. Lothaire ed., Cambridge University Press, 2002.

[107] A. Lascoux, M.-P. Schützenberger, Le monoide plaxique, Quaderni della Ricerca scientifica 109 (1981), 129-156.

[108] A. Lascoux, M.-P. Schützenberger, Structure de Hopf de l'anneau de cohomologie et de l'anneau de Grothendieck d'une variété de drapeaux, C. R. Acad. Sci. Paris Ser. I Math. 295 (1982), no. 11, 629-633.

[109] A. Lascoux, M.-P. Schützenberger, Polynômes de Schubert, C. R. Acad. Sci. Paris Sér. I Math. 294 (1982), no. 13, 447-450.

[110] A. Lascoux, M.-P. Schützenberger, Schubert polynomials and the LittlewoodRichardson rule, Lett. Math. Phys. 10(2-3) (1985), 111-124.

[111] B. Leclerc, J.-Y. Thibon, Littlewood-Richardson coefficients and Kazhdan-Lusztig polynomials, Combinatorial methods in representation theory (Kyoto, 1998), 155-220, Adv. Stud. Pure Math., 28, Kinokuniya, Tokyo, 2000.

[112] M. van Leeuwen, Edge sequences, ribbon tableaux, and an action of affine permutations, Europ. J. Combinatorics 20 (1999), 179-195.

[113] C. Lenart, Combinatorial aspects of the K-theory of Grassmannians, Ann. Combin. 4 (2000) 67-82.

[114] C. Lenart, A. Postnikov, Affine Weyl groups in K-theory and representation theory, Int. Math. Res. Not. (2007), no. 12, Art. ID rnm038, 65 pp. 
[115] C. Lenart, A. Schilling, Crystal energy functions via the charge in types $A$ and $C$, Math. Zeitschrift 273(1) (2013), 401-426.

[116] N.C. Leung, C. Li, Gromov-Witten invariants for $G / B$ and Pontryagin product for $\Omega K$, preprint arXiv:0810.4859.

[117] D. Little, Combinatorial aspects of the Lascoux-Schützenberger tree, Adv. Math. 174 (2003), no. 2, 236-253.

[118] D. E. Littlewood, A. R. Richardson, Group characters and algebra, Phil. Trans. Royal Soc. A (London) 233 (1934) 99-141.

[119] I. G. Macdonald, A new class of symmetric functions, Publ. I.R.M.A. Strasbourg, Actes $20^{e}$ Séminar Lotharingien,131-171.

[120] I. G. Macdonald, Symmetric Functions and Hall Polynomials, Second Edition, Oxford University Press, 1995.

[121] P. McNamara, Cylindric skew Schur functions, Adv. in Math. 205 (1) (2006), 275312 .

[122] K. Mészáros, private communication, October 2011.

[123] K.C. Misra, T. Miwa, Crystal base of the basic representation of $U_{q}\left(\mathfrak{s} l_{n}\right)$, Commun. Math. Phys. 134 (1990), 79-88.

[124] J. Morse, Combinatorial aspects of affine K-theory, Adv. Math. 229, No. 5 (2012), pp. 2950-2984.

[125] J. Morse, A. Schilling, A combinatorial formula for fusion coefficients, DMTCS proc AR (2012) 735-744.

[126] J. Morse, A. Schilling, Crystal operators and flag Gromov-Witten invariants, preprint

[127] D. F. Murnaghan, The Characters of the Symmetric Group, Amer. J. Math. 59 (1937), no. 4, 739-753.

[128] T. Nakashima, Crystal base and a generalization of the Littlewood-Richardson rule for the classical Lie algebras, Comm. Math. Phys. 154 (1993), no. 2, 215-243.

[129] T. Nakayama, On some modular properties of irreducible representations of symmetric groups. I and II., Jap. J. Math. 17 (1941) 411-423 and Jap. J. Math. 18 (1941) 89-108.

[130] A. Nakayashiki, Y. Yamada, Kostka polynomials and energy functions in solvable lattice models, Selecta Math. (N.S.) 3 (1997), no. 4, 547-599. 
[131] D. Peterson, Lecture Notes at MIT, 1997.

[132] S. Pon, Affine Stanley symmetric functions for classical types, J. Algebraic Combin. 36 (2012), no. 4, 595-622. and Ph.D Thesis, UC Davis, 2010.

[133] A. Postnikov, Affine approach to quantum Schubert calculus, Duke Math. J. 128 (2005), no. 3, 473-509.

[134] P. Pragacz, Algebro-geometric applications of Schur $S$ - and Q-polynomials, Topics in invariant theory (Paris, 1989/1990), 130-191, Lecture Notes in Math., 1478, Springer, Berlin, 1991.

[135] P. Pragacz, J. Ratajski, Formulas for Lagrangian and orthogonal degeneracy loci; Q-polynomial approach., Compositio Math. 107 (1997), no. 1, 11-87.

[136] D. Quillen, unpublished.

[137] A. Ram, M. Yip., A combinatorial formula for Macdonald polynomials, Adv. Math. 226 (2011), 309-331.

[138] G. de B. Robinson, On the Representations of the Symmetric Group, Amer. J. Math. 60 (3), (1938), 745-760.

[139] S. N. M. Ruijsenaars, Complete integrability of the relativistic Calogero-Moser system and elliptic function identities, Comm. Math. Phys. 110 (1987), 191-213.

[140] B. Sagan, The Symmetric Group, Graduate texts in mathematics, 203, SpringerVerlag, New York, 2001, ISBN 0387950672.

[141] The Sage-Combinat community. Sage-Combinat: enhancing Sage as a toolbox for computer exploration in algebraic combinatorics, 2008. http://combinat. sagemath.org.

[142] S. Sahi, Interpolation, integrality, and a generalization of Macdonald's polynomials, Internat. Math. Res. Notices (1996), no. 10, 457-471.

[143] C. Schensted, Longest increasing and decreasing subsequences, Can. J. Math. 13 (1961), 179-191.

[144] A. Schilling, S. O. Warnaar, Inhomogeneous lattice paths, generalized Kostka-Foulkes polynomials, and $A_{n-1}$-supernomials, Comm. Math. Phys. 202 (1999), 359-401.

[145] H. Schubert, Kalkül der abzählenden Geometrie. (German) [Calculus of enumerative geometry] Reprint of the 1879 original. With an introduction by Steven L. Kleiman. Springer-Verlag, Berlin-New York, 1979. 349 pp. ISBN: 3-540-09233-1 01A75. 
[146] M. Shimozono, A cyclage poset structure for Littlewood-Richardson tableaux, European J. Combin. 22 (2001), 365-393.

[147] M. Shimozono, Multi-atoms and monotonicity of generalized Kostka polynomials, European J. Combin. 22 (2001), 395-414.

[148] M. Shimozono, J. Weyman, Graded characters of modules supported in the closure of a nilpotent conjugacy class, European J. Combin. 21 (2000), 257-288.

[149] M. Shimozono, M. Zabrocki, Hall-Littlewood vertex operators and generalized Kostka polynomials, Adv. in Math., 158 (2001), pp. 66-85.

[150] R. Stanley, On the number of reduced decompositions of elements of Coxeter groups, European J. Combinatorics 5 (1984), 359-372.

[151] R. Stanley, Enumerative Combinatorics, Vol 2, Cambridge Studies in Advanced Mathematics, Cambridge University Press, 2001.

[152] W. A. Stein et al. Sage Mathematics Software (Version 5.4). The Sage Development Team, 2012. http://www. sagemath.org.

[153] H. Thomas, A. Yong, A jeu de taquin theory for increasing tableaux, with applications to K-theoretic Schubert calculus, Algebra Number Theory 3 (2009), no. 2, 121-148.

[154] H. Thomas, A. Yong, Longest strictly increasing subsequences, Plancherel measure and the Hecke insertion algorithm, Advances in Applied Math, to appear.

[155] D. Waugh, Upper bounds in affine Weyl groups under the weak order, Order 16 (1999), 77-87.

[156] E. Witten, The Verlinde algebra and the cohomology of the Grassmanian, "Geometry, Topology, and Physics", 357-422, Conf. Proc. Lecture Notes Geom. Topology, IV, Internat Press, Cambridge MA, 1995.

[157] M. Walton, Fusion rules in Wess-Zumino-Witten models, Nuclear Phys. B, 340 (1990), no. 2-3, 777-790.

[158] M. Zabrocki, PhD Thesis, On the action of the Hall-Littlewood vertex operator (1998).

[159] M. Zabrocki, A Macdonald vertex operator and standard tableaux statistics for the two-Column (q, t)-Kostka coefficients, Elect. J. of Comb. 5 (1998), 46 pages.

[160] A. V. Zelevinsky, Representations of finite classical groups: a Hopf algebra approach. Springer Lecture Notes, 869, (1981). 